\title{
An Overview of Acute Myeloid Leukemia and Cancer Immunology: (i) Concepts and Therapeutic Strategies and (ii) RepSox as a Candidate Cell-Engineering Tool
}

Audrey Nadine Jajosky

Follow this and additional works at: https://researchrepository.wvu.edu/etd

\section{Recommended Citation}

Jajosky, Audrey Nadine, "An Overview of Acute Myeloid Leukemia and Cancer Immunology: (i) Concepts and Therapeutic Strategies and (ii) RepSox as a Candidate Cell-Engineering Tool" (2014). Graduate Theses, Dissertations, and Problem Reports. 5874.

https://researchrepository.wvu.edu/etd/5874

This Dissertation is protected by copyright and/or related rights. It has been brought to you by the The Research Repository @ WVU with permission from the rights-holder(s). You are free to use this Dissertation in any way that is permitted by the copyright and related rights legislation that applies to your use. For other uses you must obtain permission from the rights-holder(s) directly, unless additional rights are indicated by a Creative Commons license in the record and/ or on the work itself. This Dissertation has been accepted for inclusion in WVU Graduate Theses, Dissertations, and Problem Reports collection by an authorized administrator of The Research Repository @ WVU.

For more information, please contact researchrepository@mail.wvu.edu. 


\title{
An Overview of Acute Myeloid Leukemia and Cancer Immunology:
}

(i) Concepts and Therapeutic Strategies and (ii) RepSox as a Candidate Cell-Engineering Tool

\author{
Audrey Nadine Jajosky
}

Dissertation submitted to the West Virginia University School of Medicine in partial fulfillment of the requirements for the degree of

Doctor of Philosophy

In

Cancer Cell Biology

\author{
Michael Ruppert, MD, PhD, Chair \\ Linda Vona-Davis, $\mathrm{PhD}$ \\ Steven Frisch, PhD \\ Karen Martin, $\mathrm{PhD}$ \\ William Tse, MD \\ Laura F. Gibson, PhD, mentor
}

Cancer Cell Biology Program

Morgantown, West Virginia

2014

Key words: RepSox (E 616452), acute myeloid leukemia (AML), CD34 ${ }^{+}$AML cells, Tim-3, CXCL12 (SDF-1), cancer immunotherapy, TGF- $\beta$, c-Myc, CXCR4, CXCR7, ү9ס2 T cells, reprogramming, cell engineering, co-culture, cancer stem cells, immunogenicity, immunologic synapse, aldehyde dehydrogenase (ALDH), ALK5 


\section{ABSTRACT}

\section{An Overview of Acute Myeloid Leukemia and Cancer Immunology:}

\section{(i) Concepts and Therapeutic Strategies and (ii) RepSox as a Candidate Cell-Engineering Tool}

This dissertation is intentionally broad in scope and describes the conceptual frameworks, research advances, and clinical successes that inspired a therapeutic vision that, in turn, prompted specific RepSox experiments (Jajosky, 2014). The goal of this dissertation is to guide and encourage students interested in engineering anti-cancer immune therapies by providing perspective and suggestions. Chapter I provides background on acute myeloid leukemia (AML), the cancer stem cell (CSC) theory, and the chemical reprogramming tool "RepSox." Chapter II describes how tumor cells passively evade immune recognition and actively suppress immune cells to escape destruction. Immunotherapeutic strategies are described that increase tumor-cell immunogenicity and/or sensitize tumor cells to immune-mediated death. Chapter III reviews immune-cell defects induced by cancer-distorted microenvironments. Tumor cells alter local physical and metabolic conditions and distort surrounding stromal cells in ways that impair infiltrating immune cells and promote cancer progression. Strategies are described that can reverse immune-cell defects and improve anti-tumor immunity. Chapter IV highlights successful cancer immunotherapies, including patient-specific, FDA-approved, and AML leukemic stem cell (LSC)-targeted strategies. These therapies involve antibodies, activated immune cells, and/or immuno-modulatory agents designed to eradicate tumor cells, repair and activate dysfunctional immune cells, and reduce cancer-induced immune 
suppression in tumor microenvironments. Chapter $\mathbf{V}$ describes the features and rationale of a therapeutic vision that, in combination with recent clinical and research findings, guided this project and identified specific technical obstacles. Chapter VI is the published study - entitled "RepSox slows decay of $\mathrm{CD} 4^{+}$acute myeloid leukemia cells and decreases T cell immunoglobulin mucin-3 expression" - which describes how RepSox, a "small molecule" reprogramming tool and TGF- $\beta$ inhibitor, affects AML cells. The key findings are RepSox (1) slows decay of primary CD34 ${ }^{+}$AML cells from patients with diverse AML disease, (2) increases CXCL12 and MYC, and (3) accelerates loss of Tim-3, an inhibitory (immune-checkpoint) receptor, from the surface of AML cells. Thus, RepSox may promote in vitro engineering of patient-specific AML LSC-targeted therapies by prolonging survival of primitive $\mathrm{CD} 34^{+} \mathrm{AML}$ cells and increasing AML-cell immunogenicity via Tim-3 reduction. When envisioning the immunologic synapse between antigenpresenting AML cells and T cells, the actions of RepSox suggest RepSox might promote in vitro T-cell activation against primitive (relapse-causing) AML cells which represent the most problematic therapeutic target. Chapter VII discusses the potential therapeutic applications of these results in the context of AML and other cancers as well as unanswered questions and future research options. For example, the actions of RepSox suggest that its incorporation into pre-existing co-culture methods that exploit TCR agonists may enhance the activation of $ү \delta \bar{T}$ cells against a patient's primitive AML cells and, thereby, help generate more effective immune-cell therapies. One goal of this dissertation is to encourage students to integrate new findings and conceptual frameworks from immunology, regenerative medicine, and cancer research when deciding which exciting research agendas to pursue. 


\section{DEDICATION}

This project was sustained by reviewing, as needed, fascinating stories about past cancer fighters - like those remarkable volunteers in the "Women's Field Army" (1936) immortalized by their "sword of hope" - and by receiving support from the optimistic and creative students and faculty at West Virginia University. In my future endeavors as a physician-scientist, I hope to honor the memory of NCl's Dr. Alessandra Margherita Bini whose death - by cancer - prevented me from thanking her for four years of $\mathrm{NIH}$ support.

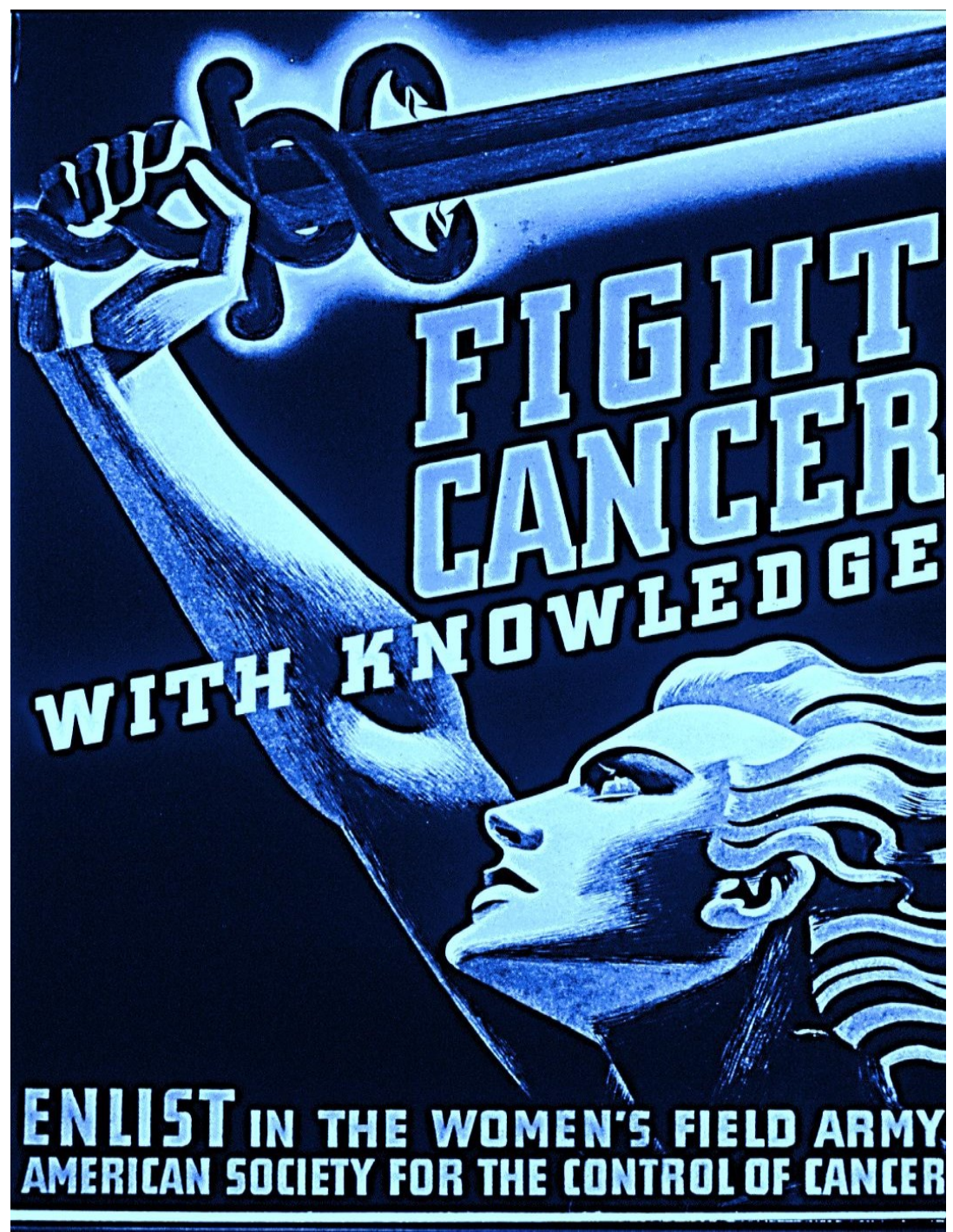




\section{ACKNOWLEDGMENTS}

Patients treated at WVU's Mary Babb Randolph Cancer Center donated their leukemia specimens so this research could be conducted. To them I am indebted, and I intend to "pay their altruism forward." My mentor, Dr. Laura Gibson, members of my PhD committee, Dr. Linda Vona-Davis, Dr. Steven Frisch, Dr. Karen Martin, Dr. Michael Ruppert, Dr. William Tse, and Dr. Fred Minnear were helpful in ways that went beyond insightful suggestions and guidance. Most notably, they fostered a creative environment that inspired me to "have no fear," design my own project, and have it critiqued by experts at the National Cancer Institute $(\mathrm{NCl})$. For four years, a sense of gratitude for the $\mathrm{NCl}$ grant award motivated me - especially when working in the lab at 3-am. Cancer researchers at $\mathrm{NCl}$ felt I had potential, and I am grateful. At WVU, the examples set by clinicians and scientists showed me how to collaborate and have fun while learning from others. Staff in the Gibson lab, WVU's Pathology Department, and flow cytometry and imaging facilities provided not just technical help but laughs and wonderful memories that make me smile. WVU's "Osborn Program" retreats reminded me that patients are the reason we work as hard as we do and that human suffering engenders a sense of urgency that can drive the creativity that helps us solve technical problems. Daniel Vanderbilt, my MD-PhD classmate, has been a wonderful friend. His constant support, commitment to science, and words of encouragement have inspired and comforted me. Overall, I am impressed by how the MD-PhD community at WVU has succeeded in creating a nurturing and creative intellectual environment. I am grateful to the patients, scientists, physicians, staff, students - and supportive neighbors - for all the fun and laughs in Morgantown, a truly exciting "college town." Go Mountaineers! 


\section{TABLE OF CONTENTS}

Dedication

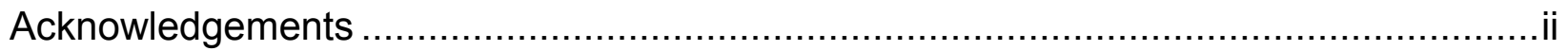

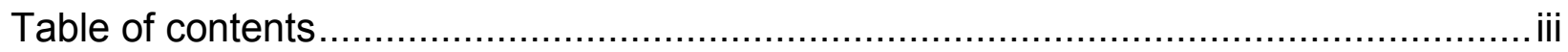

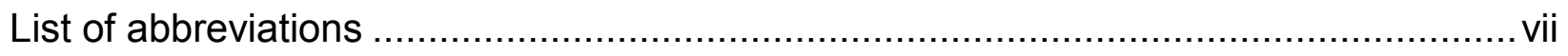

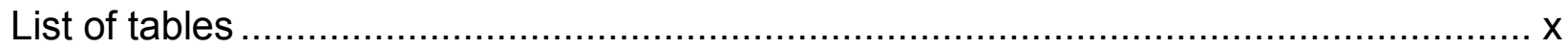

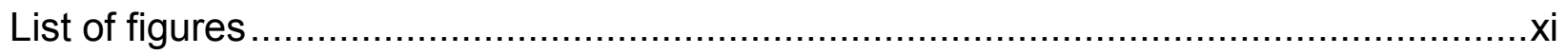

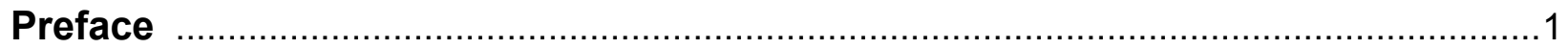

Chapter I: Background on Acute Myeloid Leukemia, the Cancer Stem Cell Theory,

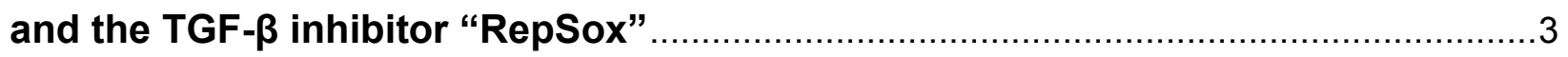

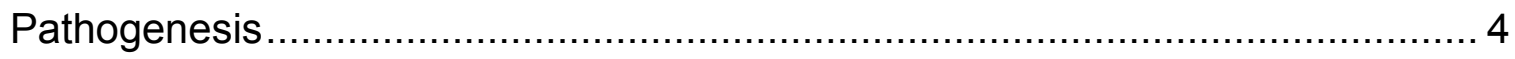

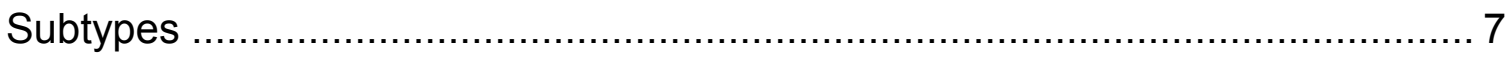

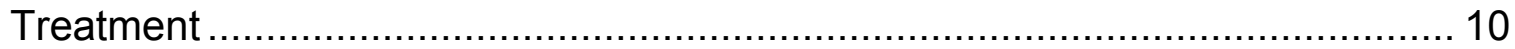

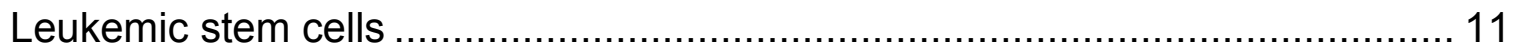

RepSox and the TGF- $\beta$ signaling pathway ............................................. 19

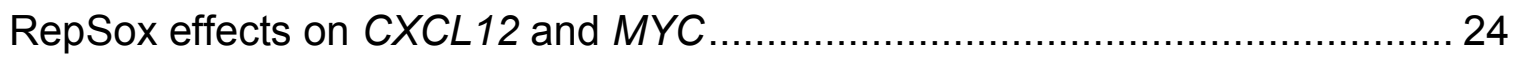

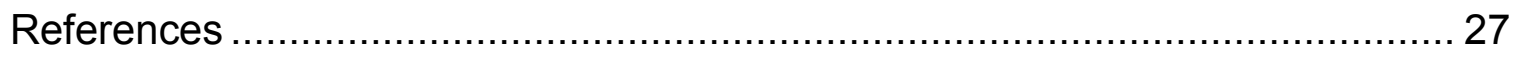

Chapter II: AML immune evasion and immune suppression; therapeutic

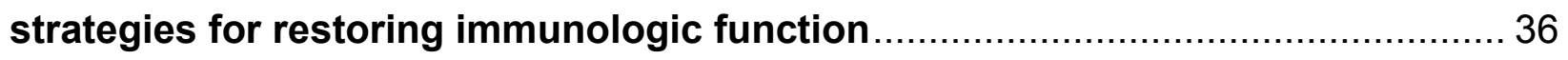

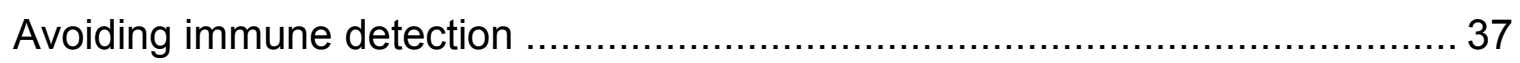

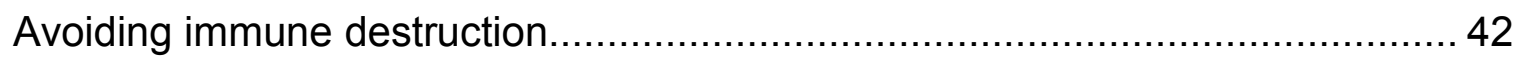


Strategies to enhance tumor-cell immunogenicity

References

Chapter III: Cancer-induced impairments of immune cells and stromal cells;

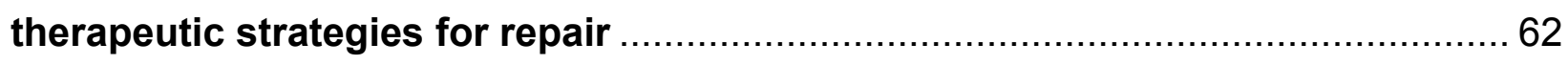

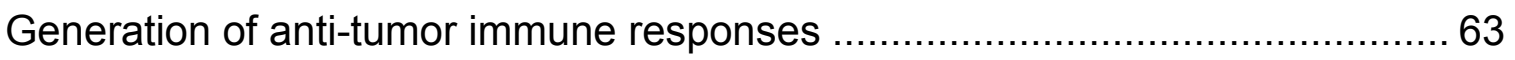

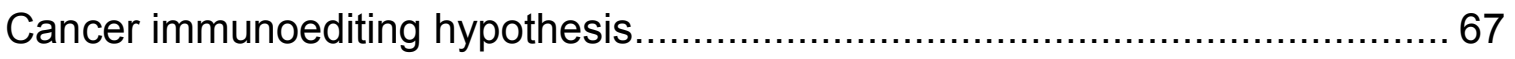

Cancer-induced dysregulation of the immune system .................................... 70

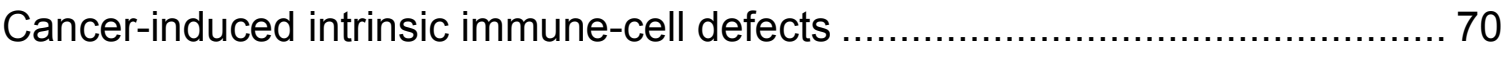

Strategies to repair dysfunctional immune cells.......................................... 72

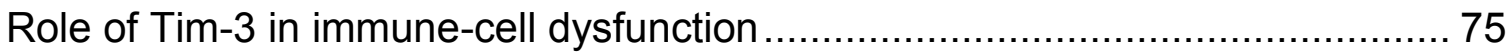

Cancer-induced extrinsic immune suppression .......................................... 78

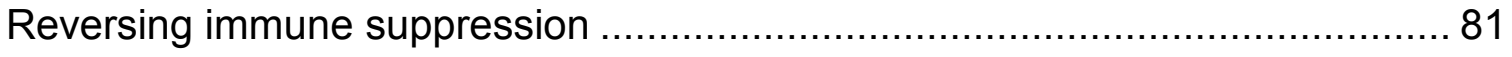

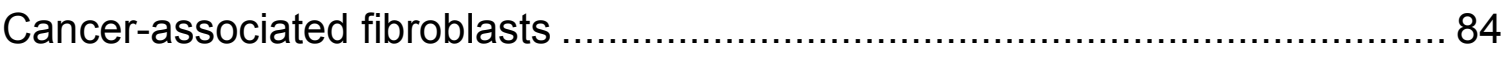

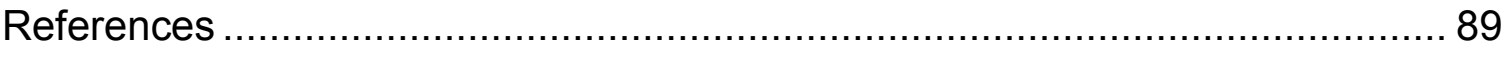

Chapter IV: Anti-cancer immunotherapy: strategies and recent successes ..... 101

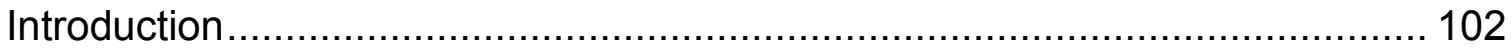

Anti-cancer immunotherapeutic strategies …............................................ 105

Adoptive transfer of immune-effector cells....................................... 105

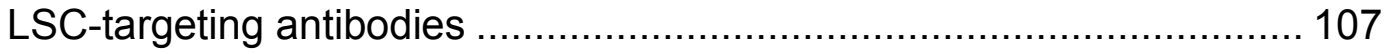

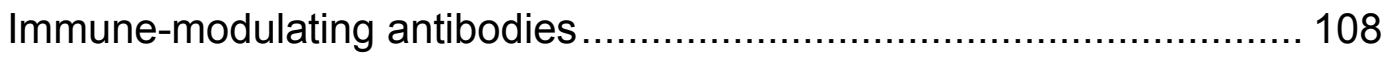

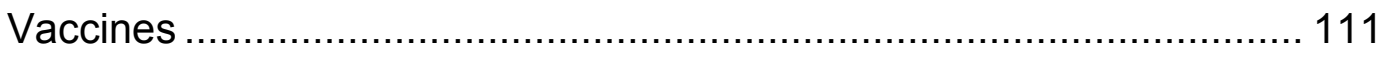




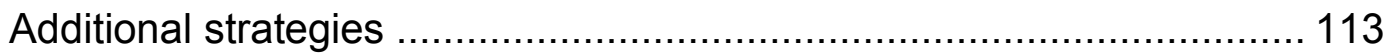

Designing and engineering patient-specific immunotherapies for AML ........... 114

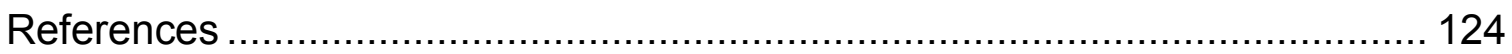

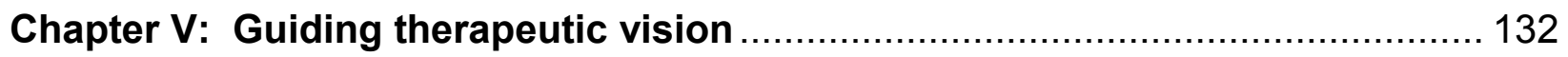

Key features of an ideal anti-cancer immunotherapeutic strategy and underlying rationale

Encouraging research findings relevant to the therapeutic vision

Role and implications of the therapeutic vision for this project

References

\section{Chapter VI: RepSox slows decay of CD34 ${ }^{+}$AML cells and decreases Tim-3}

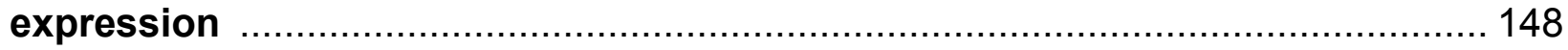

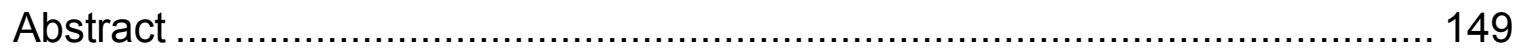

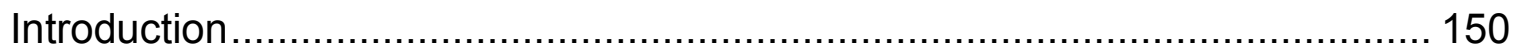

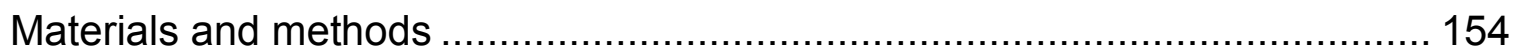

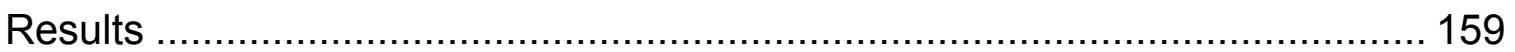

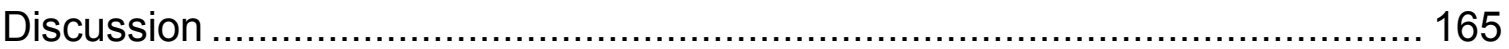

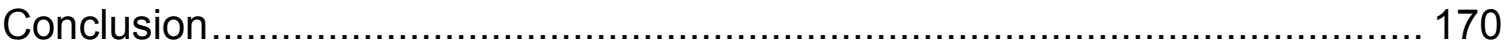

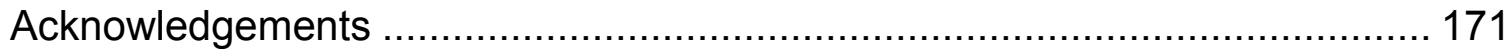

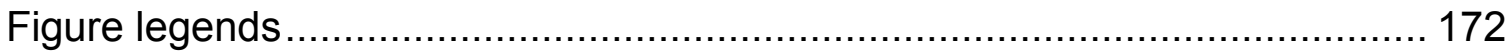

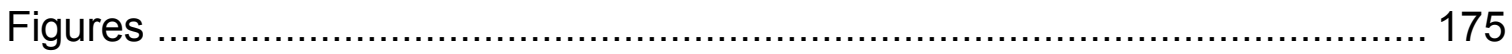

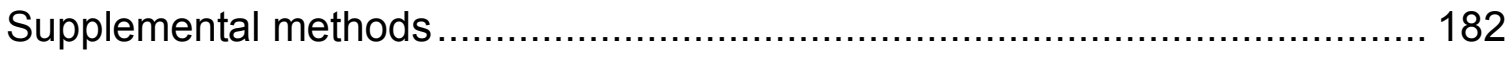


Supplemental table and figure legends

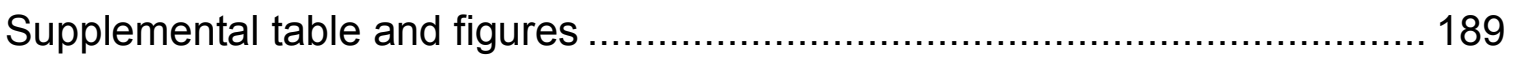

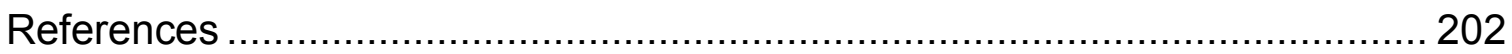

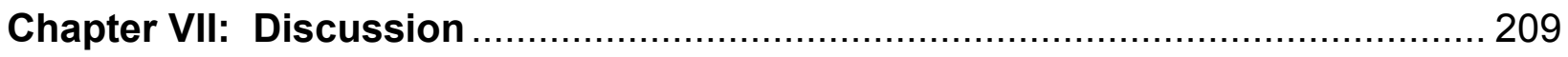

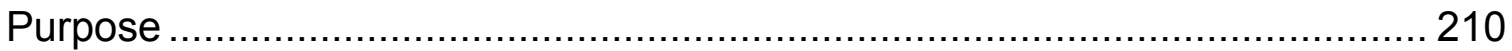

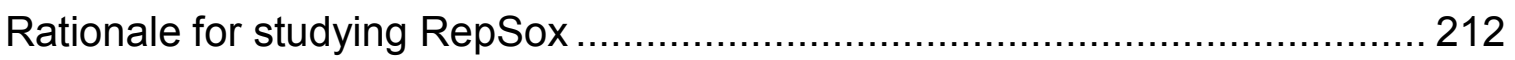

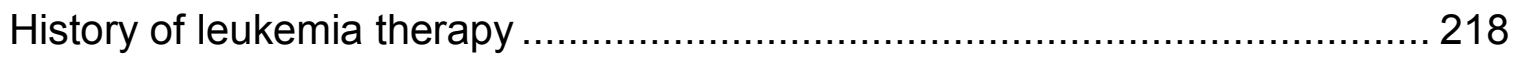

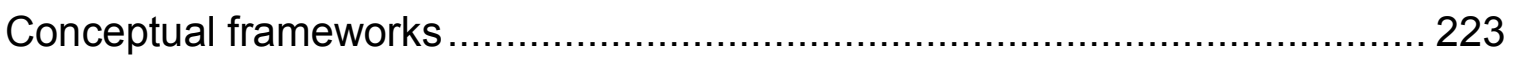

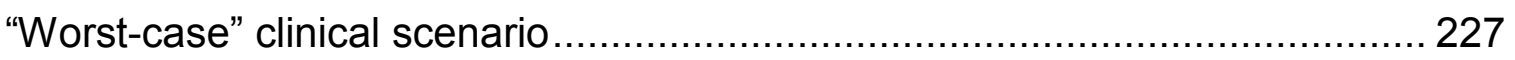

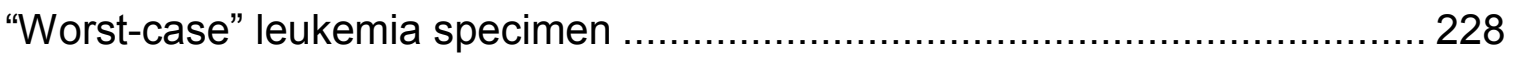

Most complex, unassuming therapy ……........................................... 236

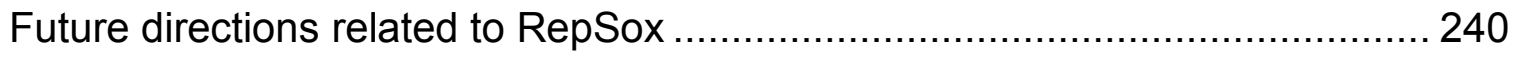

Future directions not involving RepSox ............................................... 257

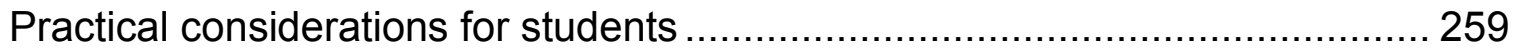

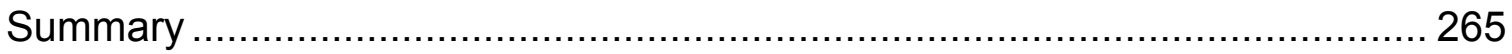

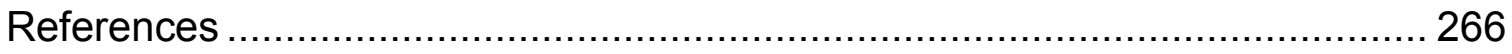

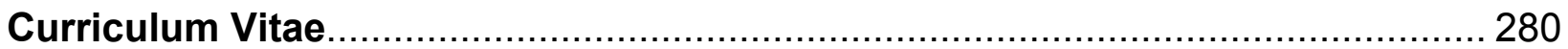




\section{LIST OF ABBREVIATIONS}

2i

3-D

DZNep

AhR

aka

ALDH

ALK5

AML

APC

ATP

ATRA

$\mathrm{Bcl}-2$

BCR/ABL

BrHPP

CAF

CAR

CBF

C/EBP

CLIP

CLL

CPP

CSC

CTLA-4

CXCL12

CXCR4

DC

DMSO

dsRNA

EMT

ETO

$\mathrm{FAB}$

FLIP

FLT3

FoxP3

G-CSF

GM-CSF

GSK3- $\beta$

GVHD

GVL

HDAC

HIV

HLA
2 inhibitor

3-dimensional

3-deazaneplanocin A

aryl hydrocarbon receptor

also known as

aldehyde dehydrogenase

activin-like receptor 5

acute myeloid leukemia

antigen-presenting cell

adenosine triphosphate

all-trans retinoic acid

B cell lymphoma 2

breakpoint cluster region/abl protein tyrosine kinase

bromohydrin pyrophosphate

cancer-associated fibroblast

chimeric antigen receptor

core binding factor

CCAAT-enhancer-binding proteins

class II-associated invariant chain peptide

chronic lymphocytic leukemia

cell-penetrating protein

cancer stem cell

cytotoxic $\mathrm{T}$ lymphocyte antigen 4

$\mathrm{C}-\mathrm{X}-\mathrm{C}$ motif chemokine 12

chemokine $\mathrm{C}-\mathrm{X}-\mathrm{C}$ motif receptor 4

dendritic cell

dimethyl sulfoxide

double-stranded RNA

epithelial to mesenchymal transition

eight twenty one

French-American-British

FLICE (FADD-like IL-1 $\beta$-converting enzyme) inhibitory protein

fms-related tyrosine kinase 3

forkhead box P3

granulocyte colony-stimulating factor

granulocyte macrophage colony-stimulating factor

glycogen synthase kinase 3 beta

graft-versus-host disease

graft-versus-leukemia

histone deacetylase inhibitor

human immunodeficiency virus

human leukocyte antigen 


\begin{tabular}{|c|c|}
\hline $\begin{array}{l}\text { HMGB1 } \\
\text { HOXA9 }\end{array}$ & $\begin{array}{l}\text { high mobility group box } 1 \\
\text { homeobox A9 }\end{array}$ \\
\hline HOXA10 & homeobox A10 \\
\hline $\mathrm{HPC}$ & hematopoietic progenitor cell \\
\hline HRG & histidine-rich glycoprotein \\
\hline HSC & hematopoietic stem cell \\
\hline HSCT & hematopoietic stem cell transplant \\
\hline ICD & immunogenic cell death \\
\hline ID1 & $\begin{array}{l}\text { inhibitor of DNA binding } 1 \text {, dominant negative helix-loop- } \\
\text { helix protein }\end{array}$ \\
\hline IDO & indoleamine 2,3-dioxygenase \\
\hline IFN & interferon \\
\hline IL & interleukin \\
\hline IL3Ra & interleukin-3 receptor alpha \\
\hline iPSC & induced pluripotent stem cell \\
\hline ITD & internal tandem duplication \\
\hline JAK2 & janus kinase 2 \\
\hline LAA & leukemia-associated antigen \\
\hline LSC & leukemic stem cell \\
\hline MAPK & mitogen-activated protein kinase \\
\hline Mcl-1 & myeloid cell leukemia sequence 1 \\
\hline MDS & myelodysplastic syndrome \\
\hline MDSC & myeloid-derived suppressor cell \\
\hline MEF & mouse embryonic fibroblast \\
\hline MET & mesenchymal to epithelial transition \\
\hline MHC & major histocompatibility complex \\
\hline MICA & MHC class I polypeptide-related sequence A \\
\hline MICB & MHC class I polypeptide-related sequence $B$ \\
\hline miRNA & microRNA \\
\hline MLL & mixed lineage leukemia \\
\hline MRD & minimal residual disease \\
\hline mRNA & messenger RNA \\
\hline mTOR & mammalian target of rapamycin \\
\hline $\mathrm{NCl}$ & National Cancer Institute (USA) \\
\hline $\mathrm{NIH}$ & National Institutes of Health (USA) \\
\hline NK & natural killer \\
\hline NKT & natural killer $\mathrm{T}$ \\
\hline NKG2D & natural killer group 2 member D \\
\hline NPM1 & nucleophosmin \\
\hline $\mathrm{P} 2 \mathrm{R} \times 7$ & purinergic receptor $\mathrm{P} 2 \mathrm{X}$ ligand-gated ion channel 7 \\
\hline PD1 & programmed cell death 1 \\
\hline PD1L & programmed cell death 1 ligand \\
\hline PDGFR $\beta$ & platelet-derived growth factor receptor beta \\
\hline PI3K & phosphatidylinositide 3-kinase \\
\hline Poly(I:C) & polyinosinic polycytidylic acid \\
\hline PRAME & preferentially expressed antigen in melanoma \\
\hline
\end{tabular}


RARa

RHAMM

RIG-1

RUNX1

RUNX3

$\mathrm{SAH}$

Sall4

SDF-1

SiRNA

SIRPa

Smad proteins

STAT3

STAT6

TAM

TAP

Tcl1a

TEL oncogene

TGF- $\beta$

$T_{h}$ cells

Tim-3

TLR

TNF

TRAIL

Tregs

VPA

WBC

WHO

WT1

WVU retinoic acid receptor alpha

hyaluronan-mediated motility receptor

retinoic acid-inducible gene 1

runt-related transcription factor 1

runt-related transcription factor 3

S-adenosylhomocysteine hydrolase

Sal-like 4

stromal cell-derived factor 1

small interfering RNA

signal regulatory protein alpha

homologs of the Drosophila protein "mothers against

decapentaplegia" (MAD)

signal transducer and activator of transcription 3

signal transducer and activator of transcription 6

tumor-associated macrophage

transporter associated with antigen processing

$\mathrm{T}$ cell leukemia/lymphoma $1 \mathrm{~A}$

another name for the ETV6 oncogene that encodes an ETS

family transcription factor

transforming growth factor beta

T helper cells

T cell immunoglobulin mucin-3

toll-like receptor

tumor necrosis factor

TNF-related apoptosis-inducing ligand

regulatory $T$ cells

valproic acid

white blood cell

World Health Organization

Wilms tumor 1

West Virginia University 


\section{LIST OF TABLES}

\section{Chapter I}

Table 1.1. The FAB classification of AML.

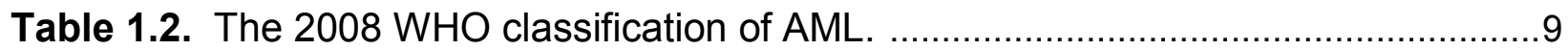

\section{Chapter VI}

Table S1. Characteristics of leukapheresis specimens. 189

\section{Chapter VII}

Table 7.1. Human cancers that express Tim-3.

Table 7.2. Tim-3-expressing immune and stromal cells in the settings of cancer and chronic viral infections. 216

Table 7.3. Potential ways to induce cellular dedifferentiation. 232

Table 7.4. Comparison of dedifferentiation processes: the two extremes. 235

Table 7.5. Measures of immune cell activation. 243 


\section{LIST OF FIGURES}

\section{Chapter I}

Figure 1.1. Cellular hierarchy within normal hematopoiesis and leukemic disease.

Figure 1.2. Reciprocal interactions between leukemic stem cells and the tumor bone

marrow microenvironment.

Figure 1.3. The TGF- $\beta$ signaling pathway.

Figure 1.4. Chemical structures of TGF- $\beta$ inhibitors.

\section{Chapter II}

Figure 2.1. Tumor cell immune escape strategies.

Figure 2.2. Processing and presentation of antigens via MHC class I molecules.

Figure 2.3. Features of immunogenic tumor cell death.

Figure 2.4. Comparison of immunosupportive and immunosuppressive tumor microenvironments.

\section{Chapter III}

Figure 3.1. Innate and adaptive immune defenses.

Figure 3.2. Generation of innate and adaptive anti-tumor immune responses. .65

Figure 3.3. Immuno-selection and immuno-subversion during tumor evolution. 68 
Figure 3.4. Three phases of cancer immunoediting: elimination, equilibrium, and escape.

Figure 3.5. Proposed mechanisms of immune suppression by immune cells and cancer-associated fibroblasts.

Figure 3.6. Role of AML-impaired stromal cells in immune suppression. .86

\section{Chapter IV}

Figure 4.1. Three-pronged anti-cancer immunotherapeutic strategy. 104

Figure 4.2. Immune checkpoint receptors and their ligands. 109

Figure 4.3. Targeting immunomodulatory receptors to improve anti-tumor immunity.

Figure 4.4. Potential "candidate tools" inspired by regeneration and cancer research that may facilitate in vitro engineering of cancer immunotherapies.

Figure 4.5. Potential ways pre-existing LSCs or in vitro-derived LSC "surrogates" may need to be manipulated for the design of LSC-targeted immunotherapies. 118

\section{Chapter V}

Figure 5.1. Timeline of significant events during thesis. 133

Figure 5.2. Patient-specific cellular immunotherapy. 136 


\section{Chapter VI}

Figure 1. Enrichment of colony-forming and $\mathrm{ALDH}^{+} \mathrm{AML}$ and MDS cells within $\mathrm{CD} 34^{+}$ fractions of leukapheresis specimens.

Figure 2. RepSox, low $\mathrm{O}_{2}$, and co-culture with $\mathrm{BM}$ stromal cells maintain $\mathrm{CD} 34^{+} \mathrm{AML}$ cells. 176

Figure 3. RepSox maintains $\mathrm{CD} 34^{+} \mathrm{AML}$ cells in a concentration-dependent manner.

Figure 4. RepSox slows decay of $\mathrm{CD} 34^{+} \mathrm{AML}$ and MDS cells.

Figure 5. RepSox-treated cells have increased CD34, CXCL12, and MYC mRNA levels and similar ALDH activity compared to DMSO-treated controls. 179

Figure 6. RepSox reversibly suppresses AML colony-forming activity. 180

Figure 7. RepSox decreases Tim-3 expression on AML and MDS cells. 181

Figure S1. Purity of $\mathrm{CD} 34^{+}$and $\mathrm{CD} 34^{-}$fractions prior to measuring colony-forming activity. 190

Figure S2. CD34 ${ }^{+} \mathrm{AML}$ and MDS cells generally display greater ALDH activity than CD34- cells.

Figure S3. RepSox does not induce CD34 expression on CD34- AML cells.

Figure S4. RepSox-treated CD34+ cells expand as effectively as DMSO controls upon cytokine stimulation. 193 
Figure S5. Generation of 3-D spheroids containing leukemic cells and osteoblasts. .194

Figure S6. RepSox inhibits TGF- $\beta$-induced phosphorylation of Smad2/3. 195

Figure S7. Unlike RepSox, additional TGF- $\beta$ inhibitors do not slow decay of CD34+ AML cells. 196

Figure S8. RepSox-treated CD $34^{+}$cells proliferate more slowly than DMSO controls.

Figure S9. Gating strategy used to select CD34+ cells. 198

Figure S10. Increased proportion of Tim $-3^{+}$cells following removal of RepSox. 199

Figure S11. TGF- $\beta$ inhibitors RepSox and SB431542 reduce Tim-3 expression on AML and MDS cells. 200

Figure S12. RepSox did not drastically alter CD47, HLA-A/B/C, or MUC1 expression on $\mathrm{CD} 34^{+} \mathrm{AML}$ and MDS cells. 201

\section{Chapter VII}

Figure 7.1. Spectrum of ways to induce de-differentiation 231

Figure 7.2. Potential mechanisms of RepSox action on AML cells. 246 


\section{PREFACE}

Deciding to write a dissertation very broad in scope is consistent with the decision made by the Journal for ImmunoTherapy of Cancer to publish in 2014 an overview entitled "Tumor immunology and cancer immunotherapy: summary of the 2013 SITC primer" authored by Raval, et al. Scientists seem to agree that the exciting anti-cancer breakthroughs and opportunities that have emerged recently should be carefully described and explained. This is an ideal time to encourage students to consider getting involved in the engineering of anti-cancer immunotherapies.

In general, researchers are now extremely optimistic, and are eager to recuit new cancer fighters. Fortunately, advances in cancer immunotherapies are truly fascinating and, when properly explained, usually generate great excitement. The pattern is encouraging: new lab observations, conceptual frameworks, and clinical findings have suggested novel anti-cancer strategies that have, in turn, prompted scientists to identify, and successfully overcome, key technical challenges. While planning this dissertation, it was decided that explaining the reasoning used by cancer researchers could be instructive. Also, to help students fully appreciate current research opportunities, an effort was made to explain the context surrounding new concepts and technical advances.

Although this dissertation is broad, readers should be able to read just the sections of interest since topics were designed to be "logically complete." Thus, redundancy was unavoidable: some of the same concepts, goals, tasks, and findings are repeated in several different locations. Of note, when discussing these topics at meetings, I found that repetition was often needed to eliminate confusion. Some concepts are, apparently, counter-intuitive or "too new or different." For example, some were confused about the 
"paradox" of working hard to keep the most lethal, stem-like leukemia cells alive. As might be expected, some immediately think about killing these lethal cells before considering how in vitro tasks that keep them alive can be used to engineer anti-LSC therapies. (Coincidentally, to develop anti-ebola therapies, scientists are trying to keep lethal ebola viruses alive in vitro.) Five points that often needed to be explained repeatedly included the following: (1) AML cells can directly function as antigen-presenting cells (when, for example, they are co-cultured with y9ס2 T cells). (2) Spontaneous de-differentiation (of normal cells) is critical for tissue regeneration in primitive animals and for sustaining human (malignant) cancer cells - while de-differentiation artificially induced in vitro can repair the dysfunctional (non-malignant) immune cells of cancer patients. (3) At any given time, a cancer patient harbors a distribution of malignant cells that includes a distribution of CSCs that evolves as the cancer progresses. That is, the phenotypes of CSCs evolve: there is not just a single, static CSC phenotype. (4) Human cancer cells are "plastic" and can de-differentiate - as well as differentiate and evolve. Thus, mature cancer cells must be eliminated as well as the CSCs. And, (5) when in vitro chemical cell-engineering is used to reprogram mature cells into pluripotent cells, some of the genes/mechanisms involved are the same as those activated during the spontaneous (and limited) cellular de-differentiation that occurs during injury-induced tissue regeneration. Overall, this dissertation tries to provide perspective and explain the rationale for this project and other research agendas. A key premise: to fully appreciate the opportunities that are exciting cancer researchers, the fascinating context for recent successes should be considered. 


\section{Chapter I}

Background on Acute Myeloid Leukemia, the Cancer Stem Cell Theory, and the TGF- $\beta$ inhibitor "RepSox" 
Acute myeloid leukemia is a white blood cell cancer involving abnormal, immature myeloid cells that rapidly proliferate, continuously evolve, and efficiently disrupt and evade immunologic attack. Accumulation of large numbers of malignant AML cells in the bone marrow suppresses normal hematopoiesis. Due to inadequate numbers of nomal white blood cells (i.e. neutrophils, macrophages), red blood cells, and platelets, AML patients may present with infections, anemia, or easy bleeding. Among the acute adult leukemias in North America and Europe, AML is the most common ( $90 \%$ of cases). Among all cancers of the elderly, AML has one of the highest mortality rates.

\section{Pathogenesis}

Leukemia may arise via transformation of normal hematopoietic stem cells or dedifferentiation of more committed blood progenitors (Passegué; 2003) as shown in Figure 1.1. Transforming events - initiated by genetic or epigenetic alterations - may bestow progenitors with stem-cell properties such as enhanced self-renewal. Regardless of cellular origin, leukemia is believed to be sustained by self-renewing leukemic stem cells (LSCs) (Lapidot, 1994; Bonnett, 1997).

Chronic myeloid leukemias often involve activating mutations in tyrosine kinases, such as BCR/ABL, TEL/PDGFR $\beta$, TEL/ABL, and TEL/JAK2 (Deguchi, 2002). In contrast, acute leukemias usually involve chromosomal alterations or mutations of transcription factors or transcriptional regulators, such as core binding factor (CBF) comprised of runt-

related transcription factor 1 (RUNX1) and $C B F \beta$ subunits, retinoic acid receptor $\alpha$ (RARa), and the mixed lineage leukemia (MLL) gene (Deguchi, 2002). Genetic lesions often occur in transcription factors that regulate hematopoiesis, leading to maturation arrest of myeloid progenitors. 


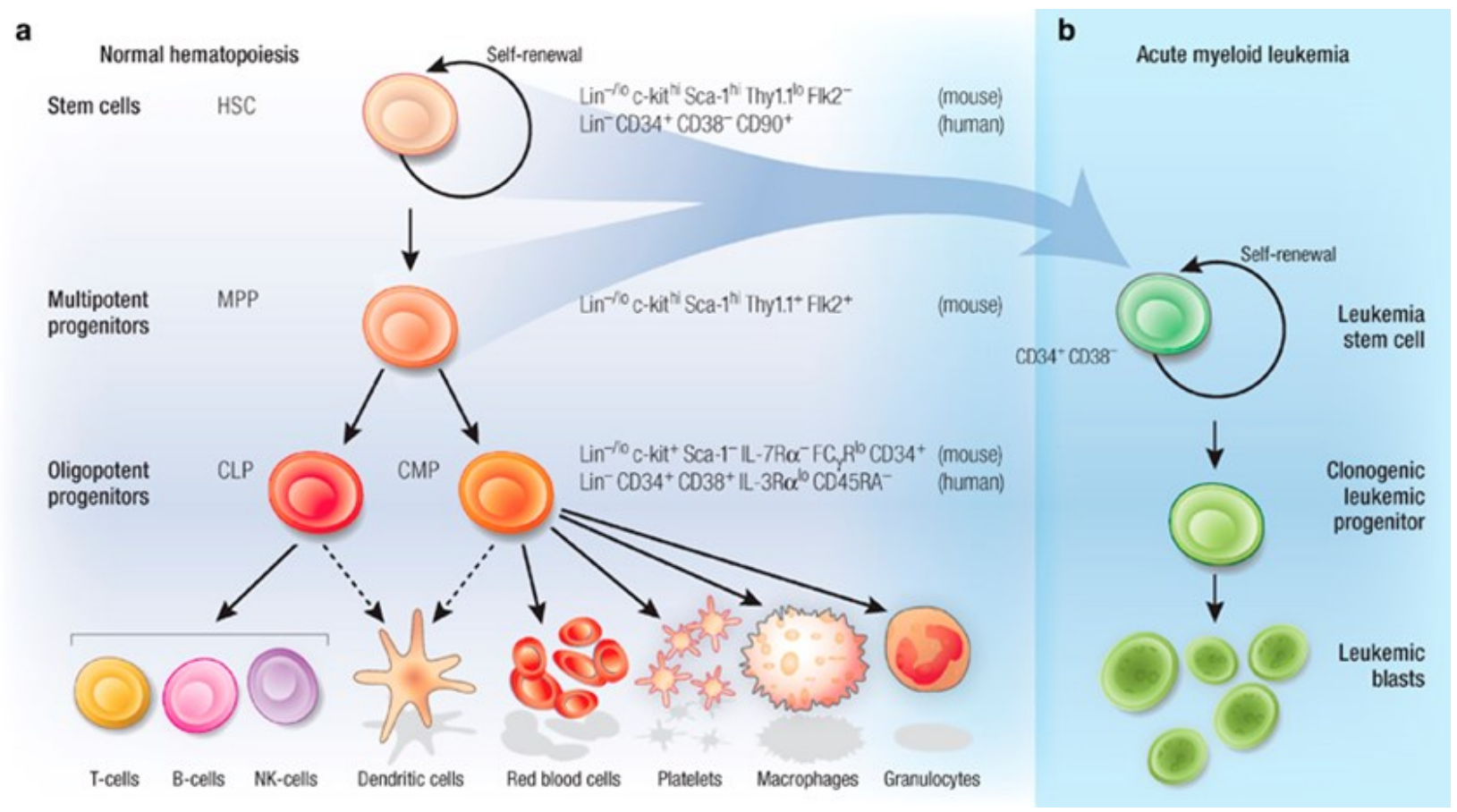

Figure 1.1. Cellular hierarchy within normal hematopoiesis and leukemic disease. During normal blood cell development, self-renewing hematopoietic stem cells (HSCs) give rise to progressively lineage-restricted progenitor cells whose progeny generate mature white blood cells, red blood cells, and platelets. Acute myeloid leukemia (AML) may arise from leukemic transformation of normal HSCs or restricted progenitor cells. According to the cancer stem cell (CSC) theory, AML is sustained by self-renewing leukemic stem cells (LSCs) that ultimately give rise to more mature leukemic blasts. Reproduced with permission from: Tan, B. T., Park, C. Y., Ailles, L. E., et al. (2006). The cancer stem cell hypothesis: a work in progress. Laboratory Investigation, 86(12), 1204. 


\section{Two-hit model of AML}

Gilliland and colleagues proposed the 2-hit model of AML in which leukemic transformation involves cooperation between two types of mutations: class I mutations that promote growth and/or survival and class II mutations that block differentiation and/or enhance self-renewal (Dash, 2001; Gilliland, 2001). Class II mutations generally occur early and are believed to help initiate disease, while class I mutations occur late and likely promote leukemic progression. Class I mutations activate signal-transduction pathways often through constitutive activation of tyrosine kinase surface receptors such as fmsrelated tyrosine kinase 3 (FLT3) and c-KIT (Gilliland, 2001). In contrast, class II mutations affect transcription factors or other transcriptional regulators. Examples of class II alterations include overexpression of homeobox (HOX) genes, mutations in hematopoietic transcription factors such as C/EBPa, MLL gene rearrangements, and creation of fusion genes such as $\mathrm{t}(8 ; 21)$ RUNX1-ETO, $\mathrm{t}(15 ; 17) \mathrm{PML}-\mathrm{RARa}$, and inv(16) CBF $\beta-M Y H 11$ (Gilliland, 2001). Study of human disease and experimental evidence from mouse models support the 2-hit hypothesis. Combinations of class I and class II mutations, such as internal tandem duplication of the FLT3 receptor (FLT3-ITD) and nucleophosmin (NPM1) mutation, coexist in AML patients (Golub, 1995; Reilly, 2005; Matsuno, 2003). Mouse studies show that hematopoietic progenitors carrying either class I or class II mutations often trigger aberrant myeloid development or myeloproliferative disorders (Kelly, 2002), while both mutations cooperate to generate leukemia (Schessl, 2005; Grisolano, 2003). 


\section{Subtypes}

$\mathrm{AML}$ is a heterogeneous disease arising from a variety of pathogenic mechanisms. Along with clinical history, AML is diagnosed by examining the morphology, cytochemistry, immunophenotype, and molecular genetics of cells in the bone marrow. According to the French-American-British (FAB) classification system (see Table 1.1), AML is divided into nine subtypes based on the myeloid lineage(s) involved and their degree of differentiation. The newer World Health Organization (WHO) classification system (see Table 1.2) also incorporates cytogenetic and molecular genetic features which provide additional prognostic value (Swerdllow, 2008). The "Immunoscore," another classification system under development, proposes that the immune-cell infiltrate within a patient's tumor can predict prognosis and response to therapy (Galon, 2012; Fridman, 2012). 
The French-American-British (FAB) Classification of AML and Associated Genetic Abnormalities

\begin{tabular}{|c|c|c|c|c|c|c|}
\hline \multirow[t]{2}{*}{$\begin{array}{l}\text { FAB } \\
\text { SuBTYPE }\end{array}$} & \multirow[t]{2}{*}{$\begin{array}{l}\text { Common Name } \\
\text { (\% of Cases) }\end{array}$} & \multicolumn{3}{|c|}{ Results of Staining } & \multirow{2}{*}{$\begin{array}{l}\text { Associated } \\
\text { TransLocations AND } \\
\text { REARRANGEMENTS } \\
\text { (\% OF CASES) }\end{array}$} & \multirow[t]{2}{*}{ Genes InVolved } \\
\hline & & $\begin{array}{l}\text { MYELOPER- } \\
\text { OXIDASE }\end{array}$ & $\begin{array}{l}\text { SUDAN } \\
\text { BLACK }\end{array}$ & $\begin{array}{l}\text { NONSPECIFIC } \\
\text { ESTERASE }\end{array}$ & & \\
\hline M0 & $\begin{array}{l}\text { Acute myeloblastic leukemia with mini- } \\
\text { mal differentiation }(3 \%)\end{array}$ & - & - & $-{ }^{*}$ & $\begin{array}{l}\operatorname{inv}(3 q 26) \text { and } \\
t(3 ; 3)(1 \%)\end{array}$ & EVII \\
\hline Ml & $\begin{array}{l}\text { Acute myeloblastic leukemia without } \\
\text { maturation }(15-20 \%)\end{array}$ & + & + & - & & \\
\hline M2 & $\begin{array}{l}\text { Acute myeloblastic leukemia with matu- } \\
\text { ration }(25-30 \%)\end{array}$ & + & + & - & $\begin{array}{l}\mathrm{t}(8 ; 21)(40 \%) \\
\mathrm{t}(6 ; 9)(1 \%)\end{array}$ & $\begin{array}{l}\text { AMLI-ETO, } \\
\text { DEK-CAN }\end{array}$ \\
\hline M3 & $\begin{array}{l}\text { Acute promyelocytic leukemia } \\
(5-10 \%)\end{array}$ & + & + & - & $\begin{array}{l}t(15 ; 17)(98 \%) \\
\quad t(11 ; 17)(1 \%) \\
t(5 ; 17)(1 \%)\end{array}$ & $\begin{array}{l}\text { PML-RAR } \alpha, P L Z F- \\
\quad R A R \alpha, N P M R A R \alpha\end{array}$ \\
\hline M4 & Acute myelomonocytic leukemia ( $20 \%)$ & + & + & + & $\begin{array}{l}11 \mathrm{q} 23(20 \%) \text {, } \\
\text { inv }(3 \mathrm{q} 26) \text { and } \\
\mathrm{t}(3 ; 3)(3 \%) \text {, } \\
\mathrm{t}(6 ; 9)(1 \%)\end{array}$ & $M L L, D E K-C A N, E V I I$ \\
\hline M4Eo & $\begin{array}{l}\text { Acute myclomonocytic leukemia with } \\
\text { abnormal cosinophils } \\
(5-10 \%)\end{array}$ & + & + & + & $\begin{array}{l}\operatorname{inv}(16), t(16 ; 16) \\
\quad(80 \%)\end{array}$ & CВF及-MYHII \\
\hline M5 & $\begin{array}{l}\text { Acute monocytic leukemia } \\
(2-9 \%)\end{array}$ & - & - & + & $\begin{array}{r}11 \mathrm{q} 23(20 \%) \\
\mathrm{t}(8 ; 16)(2 \%)\end{array}$ & $M L L, M O Z-C B P$ \\
\hline M6 & Erythroleukemia $(3-5 \%)$ & + & + & - & & \\
\hline M7 & $\begin{array}{l}\text { Acute megakaryocytic leukemia } \\
(3-12 \%)\end{array}$ & - & - & $+t$ & $\mathrm{t}(1 ; 22)(5 \%)$ & Unknown \\
\hline
\end{tabular}

${ }^{*}$ Cells are positive for myeloid antigen (e.g., CD13 and CD33).

†Cells are positive for $\boldsymbol{\alpha}$-naphthylacetate and platelet glycoprotein IIb/IIIa or factor VIII-related antigen and negative for naphthylbutyrate.

Table 1.1. The FAB classification of AML. Reproduced with permission from: Lowenberg, B., Downing, J. R., \& Burnett, A. (1999). Acute myeloid leukemia. New England Journal of Medicine, 341(14), 1052. Copyright Massachusetts Medical Society. 
AML with recurrent genetic abnormalities

AML with $\mathrm{t}(8 ; 21)(\mathrm{q} 22 ; \mathrm{q} 22) ;$ RUNX1-RUNX1T1

AML with inv(16)(p13,1q22) or t(16;16)(p13.1;q22); CBFB-MYH11

AML with $\mathrm{t}(15 ; 17)(\mathrm{q} 22 ; \mathrm{q} 12) ;$ PML-RARA

AML with $\mathrm{t}(9 ; 11)(\mathrm{p} 22 ; \mathrm{q} 23) ;$ MLLT3-MLL

AML with $\mathrm{t}(6 ; 9)(\mathrm{p} 23 ; \mathrm{q} 34) ;$ DEK-NUP214

AML with inv(3)(q21q26.2) or t(3;3)(q21;q26.2); RPN1-EVI1

AML (megakaryoblastic) with $\mathrm{t}(1 ; 22)(\mathrm{p} 13 ; \mathrm{q} 13) ;$ RBM15-MKL1

Provisional entity: AML with mutated NPM1

Provisional entity: AML with mutated CEBPA

\section{AML with myelodysplasia-related changes}

Therapy-related myeloid neoplasms

AML, not otherwise specified

AML with minimal differentiation

AML without maturation

AML with maturation

Acute myelomonocytic leukemia

Acute monoblastic/monocytic leukemia

Acute erythroid leukemias

Pure erythroid leukemia

Erythroleukemia, erythroid/myeloid

Acute megakaryoblastic leukemia

Acute basophilic leukemia

Acute panmyelosis with myelofibrosis

Table 1.2. The 2008 WHO classification of AML. Reproduced with permission from: http://www.cancernetwork.com/articles/acute-leukemias/page/0/2 


\section{Treatment}

Standard treatment for AML includes induction chemotherapy followed by consolidation therapy. The goal of induction chemotherapy is to eradicate the majority of leukemic blasts and induce remission. With the standard " $7+3$ " induction regimen of cytarabine for 7 days along with daunorubicin for the first 3 days, $70-80 \%$ of younger $(<60)$ and $50 \%$ of older (> 60) adults achieve complete remission (Lowenberg, 1999). The goal of consolidation therapy - intensive chemotherapy, such as high dose cytarabine, or hematopoietic stem cell transplant (HSCT) - is to eliminate "residual" leukemia cells and, thereby, prevent relapse. If patients cannot tolerate additional rounds of intensive chemotherapy, they may receive a HSCT. Sources of HSCs for transplant include the bone marrow or mobilized peripheral blood of the patient (autologous) or a donor (allogeneic). With allogeneic-HSCT, a post-remission strategy with the lowest rate of relapse, donor T cells attack residual leukemia cells via a graft-versus-leukemia ( $G v L$ ) immune reaction (Thomas, 1999; Jenq, 2010). A major complication of allogeneic HSCT is graft-versus-host disease (GvHD) (Thomas, 1999). Acute promyelocytic leukemia (APL; also known as M3 subtype), characterized by a translocation between chromosomes 15 and 17 [t(15;17)], is unique in its treatment with all-trans retinoic acid (ATRA) which induces APL cells to mature (Tallman, 1997).

Although more than half (50\%-70\%) of AML patients achieve complete remission after induction chemotherapy, the majority (70\%-80\%) will relapse and die of disease (Lowenberg, 1999; Döhner, 2010). Patients with poor prognosis, or those who fail to respond to standard therapy, may undergo experimental therapies in clinical trials. Immunotherapies (discussed in Chapter IV) may be most effective in the setting of minimal residual disease (MRD) when leukemic disease burden is low. Of note, LSCs 
are often enriched during MRD (Gerber, 2012), suggesting they are more likely to escape conventional chemotherapy than bulk leukemic blasts - potentially due to their quiescent nature (Terpstra, 1996) or the activity of drug efflux pumps (Wulf, 2001). Thus, by design, immunotherapies should target both the LSC population that sustains disease and the more mature leukemic blast population which (like melanoma cells) may be able to dedifferentiate into stem-like tumor cells when exposed to therapeutic stresses or microenvironmental stresses like chronic inflammation (Landsberg, 2012). Beyond initial control of disease, immune strategies have the potential to provide "cures" via generation of memory T and B cells that can provide long-term protection (Kalos, 2011; Porter, 2011).

\section{Leukemic stem cells (LSCs)}

Background

Cancer stem cells (CSCs) were first identified in AML (Lapidot, 1994). Similar to normal hematopoiesis, AML is arranged as a hierarchy originating from LSCs (aka "leukemia-initiating cells") that self-renew and give rise to more mature leukemic blasts (Bonnett, 1997; Hope, 2004). Experimentally, only a small subset of AML cells generates colonies in vitro (McCulloch, 1983; Griffin, 1986) and initiates disease when transplanted into immunocompromised mice (Lapidot, 1994; Bonnett, 1997). These findings support a hierarchical organization of AML, similar to normal hematopoiesis (Bonnett, 1997; Hope, 2004). In mice, disease-initiating cells generate the diverse spectrum of AML cells found in patients and can reconstitute disease in secondary recipients, demonstrating a capacity for self-renewal (Lapidot, 1994; Bonnett, 1997). Based on clonal tracking studies that identify cells descending from a single progenitor, the LSC compartment has been shown to contain subsets of LSCs which differ in self-renewal potential (Hope, 2004). 
Like normal HSCs, LSCs can display short-term or long-term repopulating capacity/potential (Hope, 2004). Therapeutically targeting LSCs in vivo is challenging: LSC populations may be quiescent (Terpstra, 1996), poorly immunogenic (Costello, 1999), immune- and death-resistant (van Stijn, 2003; Barrett, 2010; Lion, 2012), and phenotypically diverse (Eppert, 2011; Goardon, 2011). Furthermore, in response to stresses imposed by the tumor microenvironment, immune system, or cancer therapies, mature leukemia cells may de-differentiate into LSCs. Within any given AML patient, multiple pools of LSCs which differ in molecular and phenotypic traits may coexist (Goardon, 2011). Cancer-cell diversity and plasticity suggest that, for some patients, combination therapies may be needed to (1) attack both LSCs and more mature leukemic blasts, (2) target multiple tumor antigens (to prevent emergence of escape variants), and (3) reverse tumor-cell immune evasion, immune-system suppression, and cancerinduced dysregulations of the tumor microenvironment.

LSCs dynamically interact with the surrounding bone marrow niche, as shown in Figure 1.2. LSCs alter local physical and metabolic conditions, convert stromal cells into cancer-associated fibroblasts (CAFs), suppress infiltrating immune cells, and recruit immunosuppressive cells that support the growth and survival of LSCs by, for example, promoting "immune escape." 


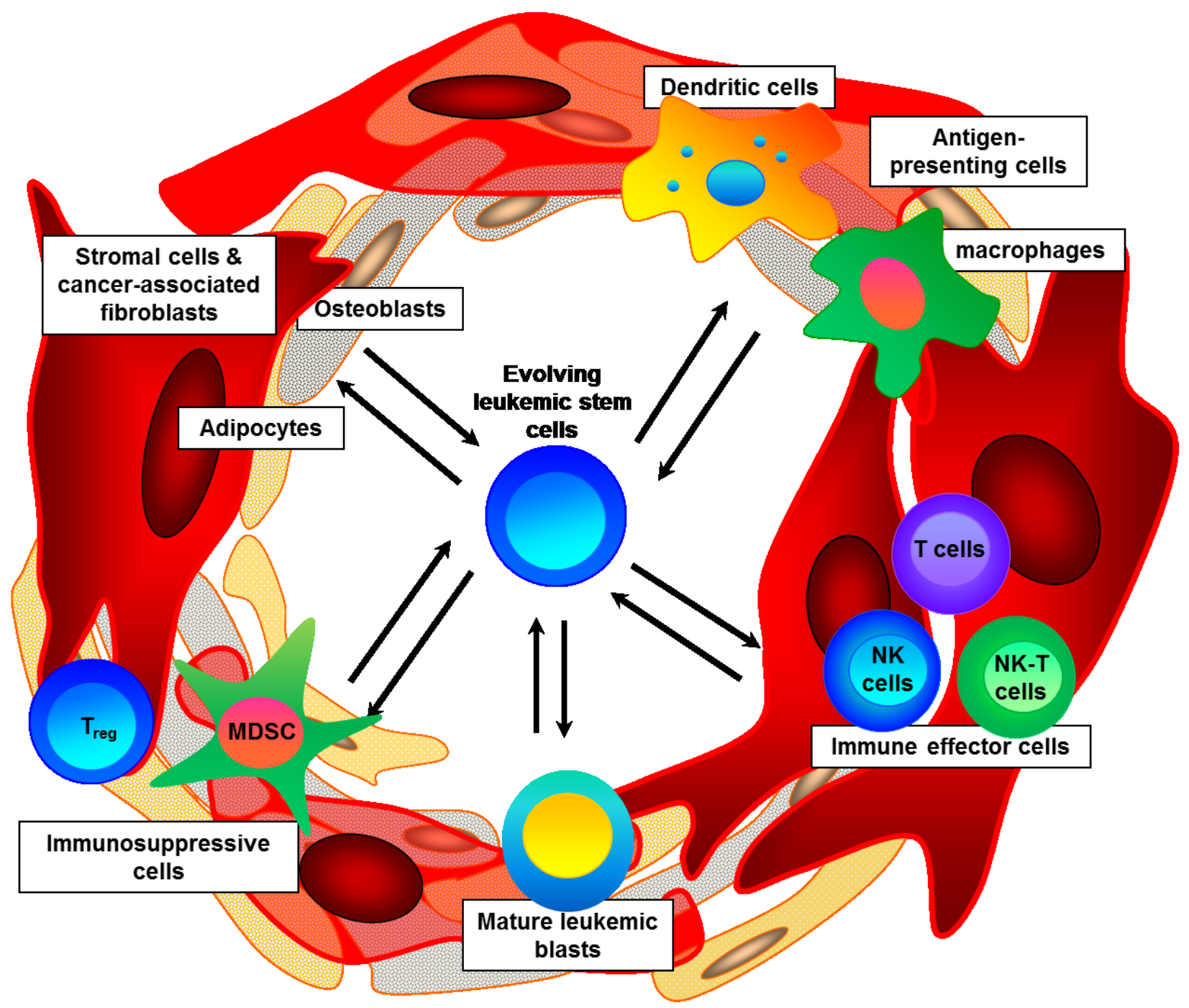

Figure 1.2. Reciprocal interactions between leukemic stem cells and the tumor bone marrow microenvironment. Within the bone marrow microenvironment, leukemia cells alter physical and metabolic conditions as well as interact with surrounding stromal cells and infiltrating immune cells. Leukemic cells deplete local oxygen and nutrients, convert stromal cells into tumor-promoting CAFs, and suppress infiltrating immune cells to promote disease progression. In reaction to microenvironmental stresses like immune selection pressures, more mature leukemia cells may dedifferentiate into LSCs as a prosurvival mechanism. The bone marrow microenvironment also nurtures leukemic cells and protects them from chemotherapy. 


\section{Clinical significance}

LSCs that survive conventional chemotherapy may trigger relapse of disease. Clinical observations support the CSC theory of AML: $C D 34^{+} C D 38-A L D H^{\text {int }} A M L$ cells were enriched during MRD, and persistence of this AML subset after induction chemotherapy was highly predictive of patients who would subsequently relapse (Gerber, 2012). AML LSC gene expression signatures generated by independent research groups correlate with patient outcomes (Eppert, 2011; Gentles, 2010). Using a signature of 52 genes upregulated in $\mathrm{CD} 34^{+} \mathrm{CD} 38^{-}$LSC-enriched fractions (as opposed to more differentiated $\mathrm{CD} 34^{+} \mathrm{CD} 38^{+}$and $\mathrm{CD} 34^{-}$fractions), high LSC scores were associated with worse overall, event-free, and disease-free survival than low LSC scores in multiple AML patient cohorts (Gentles, 2010). Rather than relying solely on immunophenotype, Eppert and colleagues generated a 42-gene LSC signature using AML LSC-enriched fractions functionally verified to initiate disease in mice (Eppert, 2011). Likewise, high LSC scores were generally associated with shorter overall and event-free survival as well as lower rates of complete remission than low LSC scores (Eppert, 2011). These studies suggest that AML LSCs are not simply artifacts of xenotransplantation but have clinical relevance. These LSC signatures may also help identify poor-risk cases of AML, drivers of LSC function, and potential LSC targets.

\section{AML LSC markers}

Across disease subtypes, disease-initiating AML LSCs were originally reported to reside exclusively within the $\mathrm{CD} 34^{+} \mathrm{CD} 38^{-}$fraction of $\mathrm{AML}$ cells when assayed in severe combined immune deficiency (SCID) mice with B- and T-cell defects and non-obese diabetic (NOD)/SCID mice carrying additional NK-cell defects (Lapidot, 1994; Bonnett, 
1997). Studies utilizing more immunodeficient mouse strains, such as NOD/SCID/IL-2 receptor $\mathrm{y}$ chain-deficient and NOD/SCID/32 microglobulin-null mice that lack NK-cell activity, show that disease-initiating LSCs can also reside within the more mature $\mathrm{CD} 34^{+} \mathrm{CD} 38^{+}$or $\mathrm{CD} 34^{-}$fractions previously believed to be non-tumorigenic (Taussig, 2008; Taussig, 2010; Sarry, 2011). Because LSC immunophenotypes vary both within (Goardon, 2011) and across (Eppert, 2011) patients, engineering of LSC-targeted therapies may require patient-specific strategies.

The discovery of cellular and molecular differences between AML LSCs and normal HSCs has identified potential therapeutic targets. Surface antigens upregulated on AML LSCs relative to normal HSCs include Tim-3 (Jan, 2011), CD47 (Majeti, 2009), CD123 (Jordan, 2000), and CD96 (Hosen, 2007) among others (Horton, 2012). Of interest, several of these receptors, including CD47 (a phagocyte "do not eat me" signal) and Tim-3 (a negative regulator of anti-tumor immunity), inhibit immune-cell functions and may contribute to the poor immunogenicity of AML LSCs (Costello, 1999; Jaiswal, 2009; Majeti, 2009). Strategies to target surface antigens using antibodies or antigen-specific immune cells, such as chimeric antigen receptor (CAR)-modified T cells, are discussed in Chapter IV.

Additional molecular differences between AML LSCs and normal HSCs include distinct patterns of ALDH staining (Pearce, 2005) as well as enhanced NFkB activity (Guzman, 2001) and apoptotic resistance (van Stijn, 2003; Yoshimoto, 2009) within LSCs. Normal HSCs are Aldefluor-bright $\left(\mathrm{ALDH}^{\mathrm{BR}}\right)$ cells, while AML LSCs display intermediate levels of Aldefluor staining (ALDH ${ }^{\text {int }}$ ) (Pearce, 2005), providing a convenient way to isolate and/or monitor both populations. Strategies to target LSCs now under 
clinical investigation include inhibitors of NFkB activity (Reikvam, 2009) and anti-apoptotic factors of the Bcl-2 family (Bose, 2013).

\section{$C D 34$}

CD34 is a single transmembrane surface glycoprotein expressed by a spectrum of normal (Sutherland, 1992; Larochelle, 1996) and leukemic (Lapidot, 1994; Bonnett, 1997) stem and progenitor cells. Experimental studies suggest CD34 can modulate cell-to-cell adhesion, enhance proliferation, and inhibit hematopoietic differentiation (Krause, 1996; Nielson, 2008). CD34 has been proposed to block the differentiation of both normal hematopoietic and leukemic progenitors (Fackler, 1995; Krause, 1996). Of note, during normal hematopoiesis, CD34 levels are highest on immature progenitors and decline with differentiation (Krause, 1996; Stella, 1995). Enforced expression of full-length CD34 on

mouse M1 leukemia cells inhibited their cytokine-induced differentiation into macrophages (Fackler, 1995). Compared to wildtype mice, CD34-knockout mice showed delayed myeloid and erythroid differentiation and decreased hematopoietic progenitors in embryonic and adult tissues (Cheng, 1996). Despite these defects, CD34-knockout mice developed normally and displayed normal mature blood cell counts (Cheng, 1996; Suzuki, 1996). Defects in myeloid and erythroid differentiation within CD34-null embryonic stem cells were reversed upon transfection with CD34 (Cheng, 1996). CD34 is expressed by $40-60 \%$ of AML patients and is most commonly observed within the more primitive M0, M1, and M2 subtypes (Borowitz, 1989). CD34 can sometimes serve as an "LSC marker" that is linked to the poor prognosis of AML patients (Geller, 1990; Myint; 1992), although conflicting results have been reported. 


\section{Tim-3}

In cancer patients, Tim-3 has been reported to be expressed by (1) tumor cells (Wiener, 2007; Jan, 2011; Kikushige, 2010), (2) dysfunctional antigen-presenting and immune-effector cells of the innate and adaptive immune system (Zhu, 2005; Anderson, 2012; Chiba, 2012; Da Silva, 2014), and (3) endothelial cells within the tumor vasculature (Huang, 2010; Wu, 2010). With the exception of acute promyelocytic leukemia (M3 subtype), Tim-3 is often expressed by myelodysplastic syndrome (MDS) and AML cells, including the CD34 ${ }^{+} \mathrm{CD} 38^{-}$subset (Kikushige, 2010; Kikushige, 2013). Tim-3 is generally upregulated upon the progression of MDS to AML, suggesting a potential role for Tim-3 in leukemogenesis (Kikushige, 2013). Because Tim-3 is not expressed by normal CD34 ${ }^{+}$CD38- hematopoietic stem and progenitor cells (Kikushige, 2010; Jan, 2011), Tim-3 receptor is an attractive therapeutic target for eliminating AML LSCs (Kikushige, 2010), restoring the functions of cancer-impaired immune cells (Zhou, 2011; Norde, 2012), and reversing immune suppression mediated by the tumor stroma (Huang, 2010).

The Tim-3 pathway may promote AML-cell survival. Galectin-9 and high mobility group box 1 (HMGB1) are the ligands of Tim-3 on lymphocytes (Zhu, 2005) and dendritic cells (DCs) (Tang, 2012). Upon toll-like receptor (TLR) stimulation, primary AML cells secrete galectin-9, and Tim-3 engagement by galectin-9 may promote survival of AML cells through upregulation of the anti-apoptotic factor myeloid cell leukemia 1 (Mcl-1) (Kikushige, 2013). Tim-3 is also expressed by a variety of solid tumors, including melanoma (Wiener, 2007), osteosarcoma (Shang, 2013), lung cancer (Zhuang, 2012), and cervical cancer (Cao, 2013) where it may promote cancer-cell immune evasion, migration, and invasion. The role of Tim-3 on cancer-impaired antigen-presenting, immune-effector, and stromal cells is reviewed in Chapter IV. 
TGF- $\beta$ has been shown to induce Tim-3 expression on mast cells (Wiener, 2007; Kim, 2012). As emphasized in this dissertation, treatment of AML cells with the TGF- $\beta$ inhibitor RepSox accelerated the decline in Tim-3 expression (Jajosky, 2014). Thus, these findings suggest a mechanism that might explain how TGF- $\beta$ can function as an immuno-suppressive cytokine in some stages of malignant disease (Gorelik, 2002; Wrzesinski, 2007).

\section{Challenges to developing LSC- targeted therapies}

Engineering anti-LSC therapies in vitro is challenging because LSCs (1) rapidly die or differentiate in culture (Pabst, 2014), (2) are poorly immunogenic (Costello, 1999; Majeti, 2009), (3) display diverse immunophenotypes (Eppert, 2011; Goardon, 2011), and (4) constitute a "moving target" as they continually evolve in vivo under constant immuneselection pressures (Horton, 2012). Furthermore, tumor microenvironments also become progressively dysregulated in ways that promote cancer cells and suppress infiltrating immune cells (Kim, 2006). Thus, LSC-targeted therapies may need to be patient-specific and administered shortly after diagnosis. By engineering therapies in vitro - outside of the immunosuppressive tumor microenvironment - it is possible to enhance immune-cell activation and reverse defects induced by cancer-distorted microenvironments. Of note, as purified Tim- $3^{+}$AML cells were cultured, levels of the immune-checkpoint receptor Tim-3 gradually declined, and RepSox accelerated this loss of Tim-3 (Chapter VI, Figure 7). In cell culture, just the removal of AML cells from the tumor microenvironment may eventually eliminate all Tim-3 expression and, in turn, increase AML-cell immunogenicity. In general, better ways to maintain a patient's LSCs in culture could facilitate a variety of valuable technical tasks: the in vitro activation of more relevant (anti-LSC) y $\delta$ 
T cells and other immune cells (by having LSCs serve as antigen-presenting cells), the identification of therapeutic targets, and the evaluation of candidate therapies preclinically. Also, when LSCs in a patient specimen are rare (or absent), it may be possible to generate LSC "surrogates" via de-differentiation of a patient's more mature ("bulk") leukemic blasts into stem-like tumor cells. By analogy, for tissue regeneration, patient-derived induced pluripotent stem cells (iPSCs) (Takahashi, 2007) may serve as artificial, lab-synthesized substitutes for naturally occurring embryonic stem cells (ESCs). LSC surrogates, chemically or genetically engineered in vitro, may serve as relevant therapeutic targets by virtue of immunologic cross-reactivity with a patient's bona fide leukemic stem and progenitor cells residing in vivo. In general, some of the same antigens that can serve as therapeutically useful targets may be expressed by a wide spectrum of leukemic progenitor cells.

\section{RepSox and the TGF- $\beta$ signaling pathway}

TGF- $\beta$ influences a variety of cellular processes including differentiation, growth, survival, embryogenesis, immune suppression, tissue fibrosis, and epithelial-tomesenchymal transition (EMT) (Li, 2006; Watabe, 2009; Wu, 2009; Xu, 2009; Massagué,

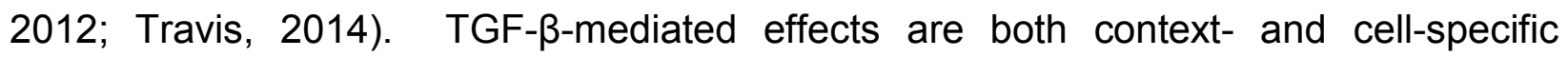
(Massagué, 2000). Figure 1.3 provides an overview of the TGF- $\beta$ signaling pathway, including TGF- $\beta$ superfamily ligands, surface receptors, and Smad proteins that regulate gene transcription (Weiss, 2013). Early in tumor development, TGF- $\beta$ generally functions as a tumor suppressor by inhibiting proliferation, promoting apoptosis, and limiting inflammation in the tumor microenvironment (Gold, 1998; Ikushima, 2010; Zammaron, 2011). In more advanced disease, however, TGF- $\beta$ facilitates cancer progression by 
promoting tumor-cell EMT, invasion, and metastasis as well as angiogenesis and immune suppression (Gold, 1998; Xu, 2009; Katsuno, 2013). 


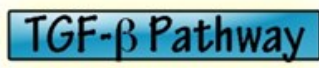

BMP Pathway

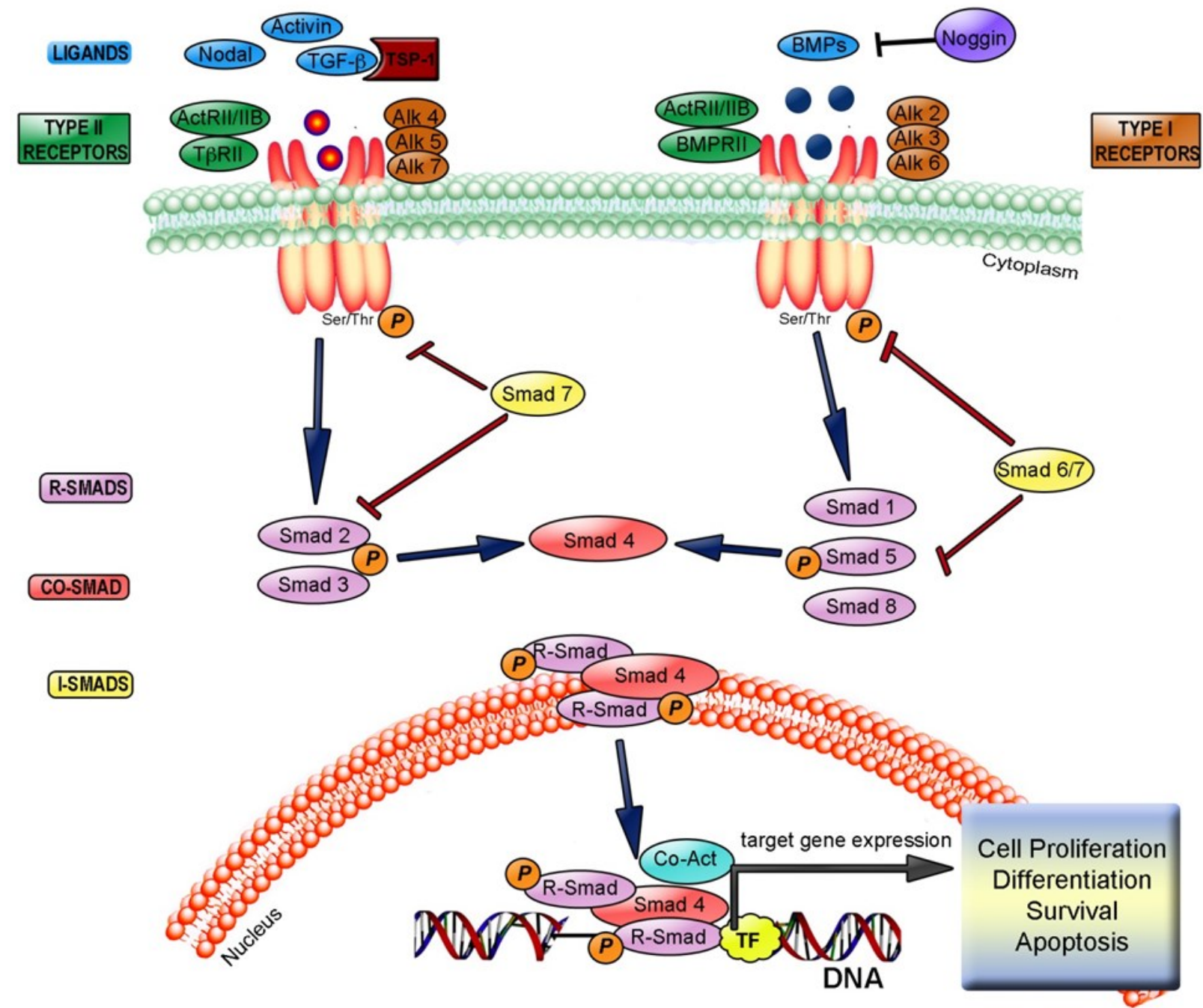

Figure 1.3. The TGF- $\beta$ signaling pathway. TGF- $\beta$ superfamily ligands (such as TGF$\beta s$, activins, nodals, and BMPs) bind to type II receptors which recruit, phosphorylate, and activate type I receptors. In the case of TGF- $\beta$ ligands, activated type I receptors phosphorylate Smad 2/3 which complexes with Smad4 and translocates to the nucleus to regulate gene transcription. RepSox selectively inhibits TGF- $\beta$ receptor $1 / A L K 5$. ( 2013 Sonia Villapol, Trevor T. Logan, Aviva J. Symes. Originally published in: "Villapol, S., Logan, T. T., \& Symes, A. J. (2013). Role of TGF- $\beta$ signaling in neurogenic regions after brain injury, chapter 1, page 5." under CC BY 3.0 license. Adapted with permission from: [Derynck, R., \& Zhang, Y. E. (2003). Smad-dependent and Smad-independent pathways in TGF- $\beta$ family signalling. Nature, 425(6958), 577-584. DOI: http://dx.doi.org/10.1038/nature02006] and [Kandasamy M, Reilmann R, Winkler J, et al. TGF- $\beta$ signaling in the neural stem cell niche: a therapeutic target for Huntington's disease. Neurology Research International. 2011. Article ID: 124256. DOI: http://dx.doi.org/10.1155/2011/124256]. Available from: http://dx.doi.org/10.5772/5394. 
RepSox, a cell-permeable, small-molecule inhibitor of TGF- $\beta$ receptor 1 [also known as activin receptor-like kinase 5 (ALK5)] (Gellibert, 2004), promotes cellular reprogramming to pluripotency by replacing Sox2 and c-Myc (Ichida, 2009) and promoting mesenchymal-to-epithelial transition (MET) (Li, 2010). Chemical structures of the other TGF- $\beta$ inhibitors studied are shown in Figure 1.4. RepSox (also known as "E-616542") is one of the seven small molecules that was used to chemically reprogram mouse fibroblasts to pluripotency - eliminating the need for genetic engineering (Hou, 2013). The other six factors used were (1) histone deacetylase (HDAC) inhibitor valproic acid (VPA), (2) glycogen synthase kinase-3 beta (GSK3- $\beta$ ) inhibitor CHIR99021, (3) monoamine oxidase inhibitor Tranylcypromine, (4) cAMP agonist Forskolin, (5) S-adenosylhomocysteine (SAH) hydrolase inhibitor 3-deazaneplanocin A (DZNep) and (6) "2i treatment" involving dual inhibition of mitogen-activated protein kinase (MAPK) signaling (using PD0325901) and GSK3- $\beta$ (using CHIR99021) (Hou, 2013). 
RepSox

(selective ALK5 inhibitor)

勧

$\mathrm{C}_{17} \mathbf{H}_{13} \mathbf{N}_{5}$

GW788388

(selective ALK5 inhibitor)

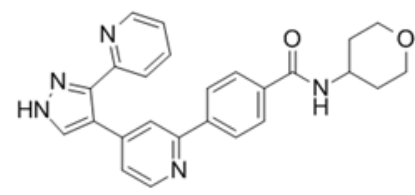

$\mathrm{C}_{25} \mathrm{H}_{23} \mathrm{~N}_{5} \mathrm{O}_{2}$
IY364947

(selective ALK5 inhibitor)<smiles>c1ccc(-c2n[nH]cc2-c2ccnc3ccccc23)nc1</smiles>

$\mathrm{C}_{17} \mathrm{H}_{12} \mathbf{N}_{4}$

(ALK4, ALK5, \& ALK7 inhibitor)

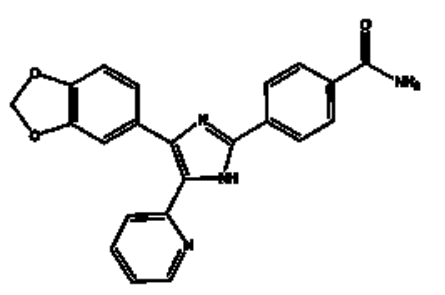

$\mathrm{C}_{22} \mathrm{H}_{16} \mathrm{~N}_{4} \mathrm{O}_{3}$

Figure 1.4. Chemical structures of TGF- $\beta$ inhibitors. RepSox is structurally most similar to LY364947; however, even among selective ALK5 TGF- $\beta$ inhibitors, we and others (Ichida, 2009) discovered effects unique to RepSox. One unique effect of RepSox's may be its ability to promote CXCL12, even across diverse cell types, including AML cells (Jajosky, 2014) and mouse embryonic fibroblasts (Larsen, 2013). 


\section{RepSox effects on CXCL12 and MYC}

In this project (Jajosky, 2014) and in other studies (Ichida, 2009) key differences between the actions of RepSox and other TGF- $\beta$ inhibitors were noted which may be due to the unique ways RepSox affects CXCL12 and c-Myc. The RepSox-induced changes in gene expression are conserved across cell types (normal fibroblasts and malignant blood cells) and animal species (mouse and human). Based on gene-expression studies, RepSox-treated MEFs expressed higher levels of C-X-C motif chemokine 12 (CXCL12) and MYC than DMSO-treated controls (Larsen, 2013). Similarly, in this study, RepSoxtreated AML cells more highly expressed CXCL12 and MYC than controls (Jajosky, 2014). Because studies suggest both c-Myc and CXCL12 inhibit differentiation of AML cells (Leon, 2009; Salvatori, 2011; Huang, 2006; Tavor, 2008), upregulation of these two factors may explain how RepSox slows decay of CD34+ AML cells (Jajosky, 2014).

CXCL12 - also known as "stromal cell-derived factor 1" (SDF-1) - is a chemokine that promotes migration and homing of normal hematopoietic progenitors (Aiuti, 1997) as well as metastasis of cancer cells (Müller, 2001) along chemotactic gradients. CXCL12 also promotes survival of AML cells (Tavor, 2014; Kim, 2013) and may help maintain them in an un-differentiated state (Tavor, 2008). Exposing primary AML cells and cell lines to the chemokine C-X-C motif receptor 4 (CXCR4) antagonist AMD3100 induced differentiation as evidenced by changes in morphology as well as expression of myeloid differentiation antigens (i.e. increased CD15, CD11b), differentiation-promoting cytokines (i.e. increased G-CSF and GM-CSF), and transcription factors (i.e. downregulation of HOXA9, HOXA10, and WT1 involved in leukemogenesis and upregulation of C/EBPE, ID1, and RUNX3 induced during myeloid differentiation) (Tavor, 2008). Furthermore, the 
balance between TGF- $\beta$ and CXCL12 signaling also influences the cell-cycle status of normal hematopoietic progenitors (Chabanon, 2008).

c-Myc is a transcription factor that facilitates reprogramming of differentiated cells toward pluripotency (Takahashi, 2006) and can inhibit the differentiation of a variety of both normal and malignant cells (Leon, 2009). In transgenic mice engineered to conditionally express c-Myc within blood cells, inactivation of c-Myc triggered differentiation of AML and lymphoma cells, leading to cancer regression (Felsher, 1999). During all-trans retinoic acid (ATRA)-induced and 1,25-dihydroxyvitamin $\mathrm{D}_{3}$-induced differentiation of AML cell lines, c-Myc levels declined (Salvatori, 2011). Furthermore, exposure to the small molecule c-Myc inhibitor "10058-F4" (which inhibits dimerization of c-Myc with transcription factor Max) induced primary AML cells to differentiate (Huang, 2006). Thus, c-Myc might help maintain AML cells in an undifferentiated state.

RepSox is a potent reprogramming tool and the actions of RepSox on normal-cell differentiation (Ichida, 2009) seem relevant for maintaining CSCs. Gene-expression studies revealed RepSox-treated MEFs expressed higher levels of components of the Wnt, Notch, and Hedgehog signaling pathways than DMSO-treated controls (Larsen, 2013). Among TGF- $\beta$ inhibitors, the unique actions of RepSox may be due to its structure (see Figure 1.4), potency, or selectivity. While RepSox promoted iPSC reprogramming in the absence of the HDAC inhibitor VPA, other TGF- $\beta$ inhibitors, such as LY364947 (or E-616451), did not (Ichida, 2009). Of note, TGF- $\beta$ neutralizing antibody did not enhance reprogramming efficiency as well as RepSox (Ichida, 2009). Among the different tools that can be used to inhibit TGF- $\beta$ signaling, RepSox may be an especially useful way to activate genes involved in stem-cell maintenance, self-renewal, and pluripotency - or to 
repress pro-differentiation genes. Comparing TGF- $\beta$ inhibitors in this project showed that RepSox most effectively (1) increased or maintained CXCL12 and MYC mRNA levels and (2) slowed decay of CD34+ AML cells (Jajosky, 2014). Of note, RepSox substitutes for c-Myc during reprogramming (Ichida, 2009), and both c-Myc and CXCL12 (aka SDF-1) are believed to inhibit the differentiation of AML cells (Huang, 2006; Tavor, 2008).

Interestingly, genes activated during limb regeneration, such as Sal-like 4 (Sall4), are also activated during cellular reprogramming to pluripotency (Neff, 2011). That is, when cell de-differentiation is ("artificially") induced in vitro, this may involve some of the same molecular mechanisms that are responsible for the de-differentiation that occurs ("naturally") in vivo when primitive animals regenerate their amputated limbs.

As with other cancers that progress, AML persists when the patient's immune system fails to eliminate the malignant cells. A combination of intrinsic tumor-derived factors as well as extrinsic cancer-induced dysfunctions of the immune system and tumor microenvironment promotes AML immune escape and disease progression. Chapter II reviews the features of cancer cells (i.e. poor antigen-presentation, low immunogenicity, death-resistance) that enable them to passively escape ("hide") and actively suppress (“combat") immune defenses. In vitro cell-engineering strategies are described that may enhance in vitro immune-cell activation (i.e. improve presentation of leukemic antigens). Multi-pronged options are described that might, for example, use systemic agents to reduce the death-resistance of leukemia cells in vivo (i.e. inhibit generation of the specific anti-apoptotic factors that render a patient's cancer cells resistant to immune-cell attack). 


\section{References}

Aiuti, A., Webb, I. J., Bleul, C., et al. (1997). The chemokine SDF-1 is a chemoattractant for human CD34+ hematopoietic progenitor cells and provides a new mechanism to explain the mobilization of CD34+ progenitors to peripheral blood. The Journal of Experimental Medicine, 185(1), 111-120.

Anderson, A. C. (2012). Tim-3, a negative regulator of anti-tumor immunity.Current Opinion in Immunology, 24(2), 213-216.

Barrett, A. J., \& Le Blanc, K. (2010). Immunotherapy prospects for acute myeloid leukaemia. Clinical \& Experimental Immunology, 161(2), 223-232.

Bonnet, D., \& Dick, J. E. (1997). Human acute myeloid leukemia is organized as a hierarchy that originates from a primitive hematopoietic cell. Nature Medicine, 3(7), 730737.

Bose, P., \& Grant, S. (2013). Mcl-1 as a therapeutic target in acute myelogenous leukemia (AML). Leukemia Research Reports, 2(1), 12-14.

Cao, Y., Zhou, X., Huang, X., et al. (2013). Tim-3 expression in cervical cancer promotes tumor metastasis. PloS One, 8(1), e53834.

Chabanon, A., Desterke, C., Rodenburger, E., et al. (2008). A cross-talk between stromal cell-derived factor- 1 and transforming growth factor- $\beta$ controls the quiescence/cycling switch of $\mathrm{CD} 34+$ progenitors through $\mathrm{FoxO} 3$ and mammalian target of rapamycin. Stem Cells, 26(12), 3150-3161.

Cheng, J., Baumhueter, S., Cacalano, G., et al. (1996). Hematopoietic defects in mice lacking the sialomucin CD34. Blood, 87(2), 479-490.

Chiba, S., Baghdadi, M., Akiba, H., et al. (2012). Tumor-infiltrating DCs suppress nucleic acid-mediated innate immune responses through interactions between the receptor TIM3 and the alarmin HMGB1. Nature Immunology, 13(9), 832-842.

da Silva, I. P., Gallois, A., Baranda, S. J., et al. (2014). Reversal of NK cell exhaustion in advanced melanoma by Tim-3 blockade. Cancer Immunology Research, 2(5), 410-422.

Dash, A., \& Gilliland, D. G. (2001). Molecular genetics of acute myeloid leukaemia. Best Practice \& Research Clinical Haematology, 14(1), 49-64.

Deguchi, K., \& Gilliland, D. G. (2002). Cooperativity between mutations in tyrosine kinases and in hematopoietic transcription factors in AML. Leukemia, 16(4), 740-744. 
Döhner, H., Estey, E. H., Amadori, S., et al. (2010). Diagnosis and management of acute myeloid leukemia in adults: recommendations from an international expert panel, on behalf of the European LeukemiaNet. Blood, 115(3), 453-474.

Eppert, K., Takenaka, K. *, Franck Pagès1,2,3,4, Francesco M Marincola5,6, Lechman, E. R., et al. (2011). Stem cell gene expression programs influence clinical outcome in human leukemia. Nature Medicine, 17(9), 1086-1093.

Fackler, M. J., Krause, D. S., Smith, O. M., et al. (1995). Full-length but not truncated CD34 inhibits hematopoietic cell differentiation of M1 cells. Blood, 85(11), 3040-3047.

Felsher, D. W., \& Bishop, J. M. (1999). Reversible Tumorigenesis by MYC in Hematopoietic Lineages. Molecular Cell, 4(2), 199-207.

Fridman, W. H., Pagès, F., Sautès-Fridman, C., \& Galon, J. (2012). The immune contexture in human tumours: impact on clinical outcome. Nature Reviews Cancer, 12(4), 298-306.

Galon, J., Pagès F., Marincola, F. M., et al. (2012). Cancer classification using the Immunoscore: a worldwide task force. Journal of Translational Medicine, 10, 1-9.

Geller, R. B., Zahurak, M., Hurwitz, C. A., et al. (1990). Prognostic importance of immunophenotyping in adults with acute myelocytic leukaemia: the significance of the stem-cell glycoprotein CD34 (My 10). British Journal of Haematology, 76(3), 340-347.

Gellibert, F., Woolven, J., Fouchet, M. H., et al. (2004). Identification of 1, 5-naphthyridine derivatives as a novel series of potent and selective TGF- $\beta$ type I receptor inhibitors. Journal of Medicinal Chemistry, 47(18), 4494-4506.

Gentles, A. J., Plevritis, S. K., Majeti, R., et al. (2010). Association of a leukemic stem cell gene expression signature with clinical outcomes in acute myeloid leukemia. Journal of the American Medical Association, 304(24), 2706-2715.

Gerber, J. M., Smith, B. D., Ngwang, B., et al. (2012). A clinically relevant population of leukemic CD34+ CD38- cells in acute myeloid leukemia. Blood, 119(15), 3571-3577.

Gilliland, D. G. (2001). Hematologic malignancies. Current Opinion in Hematology, 8(4), 189-191.

Gilliland, D. G., \& Griffin, J. D. (2002). The roles of FLT3 in hematopoiesis and leukemia. Blood, 100(5), 1532-1542.

Goardon, N., Marchi, E., Atzberger, A., et al. (2011). Coexistence of LMPP-like and GMPlike leukemia stem cells in acute myeloid leukemia. Cancer Cell, 19(1), 138-152. 
Gold, L. I. (1998). The role for transforming growth factor-beta (TGF-beta) in human cancer. Critical Reviews in Oncogenesis, 10(4), 303-360.

Golub, T. R., Barker, G. F., Bohlander, S. K., et al. (1995). Fusion of the TEL gene on $12 \mathrm{p} 13$ to the AML1 gene on 21q22 in acute lymphoblastic leukemia. Proceedings of the National Academy of Sciences, 92(11), 4917-4921.

Gorelik, L., \& Flavell, R. A. (2002). Transforming growth factor- $\beta$ in T-cell biology. Nature Reviews Immunology, 2(1), 46-53.

Griffin, J. D., \& Lowenberg, B. (1986). Clonogenic cells in acute myeloblastic leukemia. Blood, 68(6), 1185-1195.

Grisolano, J. L., O'Neal, J., Cain, J., et al. (2003). An activated receptor tyrosine kinase, TEL/PDGF $\beta R$, cooperates with AML1/ETO to induce acute myeloid leukemia in mice. Proceedings of the National Academy of Sciences, 100(16), 9506-9511.

Guzman, M. L., Neering, S. J., Upchurch, D., et al. (2001). Nuclear factor-kB is constitutively activated in primitive human acute myelogenous leukemia cells. Blood, 98(8), 2301-2307.

Hope, K. J., Jin, L., \& Dick, J. E. (2004). Acute myeloid leukemia originates from a hierarchy of leukemic stem cell classes that differ in self-renewal capacity. Nature Immunology, 5(7), 738-743.

Horton, S. J., \& Huntly, B. J. (2012). Recent advances in acute myeloid leukemia stem cell biology. Haematologica, 97(7), 966-974.

Hosen, N., Park, C. Y., Tatsumi, N., et al. (2007). CD96 is a leukemic stem cell-specific marker in human acute myeloid leukemia. Proceedings of the National Academy of Sciences, 104(26), 11008-11013.

Hou, P., Li, Y., Zhang, X., et al. (2013). Pluripotent stem cells induced from mouse somatic cells by small-molecule compounds. Science, 341(6146), 651-654.

Huang, X., Bai, X., Cao, Y., et al. (2010). Lymphoma endothelium preferentially expresses Tim-3 and facilitates the progression of lymphoma by mediating immune evasion. The Journal of Experimental Medicine, 207(3), 505-520.

Huang, M. J., Cheng, Y. C., Liu, C. R., et al. (2006). A small-molecule c-Myc inhibitor, 10058-F4, induces cell-cycle arrest, apoptosis, and myeloid differentiation of human acute myeloid leukemia. Experimental Hematology, 34(11), 1480-1489. 
Ichida, J. K., Blanchard, J., Lam, K., et al. (2009). A small-molecule inhibitor of Tgf- $\beta$ signaling replaces sox2 in reprogramming by inducing nanog. Cell Stem Cell, 5(5), 491503.

Ikushima, H., \& Miyazono, K. (2010). TGF $\beta$ signalling: a complex web in cancer progression. Nature Reviews Cancer, 10(6), 415-424.

Jaiswal, S., Jamieson, C. H., Pang, W. W., et al. (2009). CD47 is upregulated on circulating hematopoietic stem cells and leukemia cells to avoid phagocytosis. Cell, 138(2), 271-285.

Jajosky, A. N., Coad, J. E., Vos, J. A., et al. (2014). RepSox slows decay of CD34+ acute myeloid leukemia cells and decreases $\mathrm{T}$ cell immunoglobulin mucin-3 expression. Stem Cells Translational Medicine, 3(7), 836-848.

Jan, M., Chao, M. P., Cha, A. C., et al. (2011). Prospective separation of normal and leukemic stem cells based on differential expression of TIM3, a human acute myeloid leukemia stem cell marker. Proceedings of the National Academy of Sciences, 108(12), 5009-5014.

Jenq, R. R., \& Van den Brink, M. R. (2010). Allogeneic haematopoietic stem cell transplantation: individualized stem cell and immune therapy of cancer. Nature Reviews Cancer, 10(3), 213-221.

Jordan, C. T., Upchurch, D., Szilvassy, S. J., et al. (2000). The interleukin-3 receptor alpha chain is a unique marker for human acute myelogenous leukemia stem cells. Leukemia, 14(10), 1777-1784.

Kalos, M., Levine, B. L., Porter, D. L., et al. (2011). T cells with chimeric antigen receptors have potent antitumor effects and can establish memory in patients with advanced leukemia. Science Translational Medicine, 3(95), 95ra73.

Katsuno, Y., Lamouille, S., \& Derynck, R. (2013). TGF- $\beta$ signaling and epithelialmesenchymal transition in cancer progression. Current Opinion in Oncology, 25(1), 7684.

Kelly, L. M., Liu, Q., Kutok, J. L., et al. (2002). FLT3 internal tandem duplication mutations associated with human acute myeloid leukemias induce myeloproliferative disease in a murine bone marrow transplant model. Blood, 99(1), 310-318.

Kikushige, Y., Shima, T., Takayanagi, S. I., et al. (2010). TIM-3 is a promising target to selectively kill acute myeloid leukemia stem cells. Cell Stem Cell, 7(6), 708-717. 
Kikushige, Y., Yuda, J., Shima, T., et al. (2013). TIM-3, a leukemia stem cell marker, plays a role in leukemic transformation through autocrine stimulatory signaling by its ligand, galectin-9. Blood, 122(21), 4196-4196.

Kim, R., Emi, M., Tanabe, K., et al. (2006). Tumor-driven evolution of immunosuppressive networks during malignant progression. Cancer Research, 66(11), 5527-5536.

Kim, H. Y., Oh, Y. S., Song, I. C., et al. (2013). Endogenous stromal cell-derived factor-1 (CXCL12) supports autonomous growth of acute myeloid leukemia cells. Leukemia Research, 37(5), 566-572.

Kim, J. S., Shin, D. C., Woo, M. Y., et al. (2012). T cell immunoglobulin mucin domain (TIM)-3 promoter activity in a human mast cell line. Immune Network, 12(5), 207-212.

Krause, D. S., Fackler, M. J., Civin, C. I., et al. (1996). CD34: structure, biology, and clinical utility [see comments]. Blood, 87(1), 1-13.

Landsberg, J., Kohlmeyer, J., Renn, M., et al. (2012). Melanomas resist T-cell therapy through inflammation-induced reversible dedifferentiation. Nature, 490(7420), 412-416.

Lapidot, T., Sirard, C., Vormoor, J., et al. (1994). A cell initiating human acute myeloid leukaemia after transplantation into SCID mice. Nature, 367(6464), 645-648.

Larochelle, A., Vormoor, J., Hanenberg, H., et al. (1996). Identification of primitive human hematopoietic cells capable of repopulating NOD/SCID mouse bone marrow: implications for gene therapy. Nature Medicine, 2(12), 1329-1337.

Larsen, D. M. (2013). Evaluation of the TGF- $\beta$ inhibitor RepSox on the expression of pluripotency pathways in murine and bovine cells (Master's thesis). Available from ProQuest Dissertations and Theses database. (UMI Number: 1537216).

Li, R., Liang, J., Ni, S., et al. (2010). A mesenchymal-to-epithelial transition initiates and is required for the nuclear reprogramming of mouse fibroblasts. Cell Stem Cell, 7(1), 5163.

Li, M. O., Wan, Y. Y., Sanjabi, S., et al. (2006). Transforming growth factor- $\beta$ regulation of immune responses. Annual Review of Immunology, 24, 99-146.

Leon, J., Ferrandiz, N., Acosta, J. C., et al. (2009). Inhibition of cell differentiation. Cell Cycle, 8(8), 1148-1157.

Lion, E., Willemen, Y., Berneman, Z. N., et al. (2012). Natural killer cell immune escape in acute myeloid leukemia. Leukemia, 26(9), 2019-2026. 
Lowenberg, B., Downing, J. R., \& Burnett, A. (1999). Acute myeloid leukemia. New England Journal of Medicine, 341(14), 1051-1062.

Majeti, R., Chao, M. P., Alizadeh, A. A., et al. (2009). CD47 is an adverse prognostic factor and therapeutic antibody target on human acute myeloid leukemia stem cells. Cell, 138(2), 286-299.

Massagué, J. (2000). How cells read TGF- $\beta$ signals. Nature Reviews Molecular Cell Biology, 1(3), 169-178.

Massagué, J. (2012). TGF $\beta$ signalling in context. Nature Reviews Molecular Cell Biology, 13(10), 616-630.

Matsuno, N., Osato, M., Yamashita, N., et al. (2003). Dual mutations in the AML1 and FLT3 genes are associated with leukemogenesis in acute myeloblastic leukemia of the M0 subtype. Leukemia, 17(12), 2492-2499.

McCulloch, E. A. (1983). Stem cells in normal and leukemic hemopoiesis (Henry Stratton Lecture, 1982). Blood, 62(1), 1-13.

Müller, A., Homey, B., Soto, H., et al. (2001). Involvement of chemokine receptors in breast cancer metastasis. Nature, 410(6824), 50-56.

Myint, H., \& Lucie, N. P. (1992). The prognostic significance of the CD34 antigen in acute myeloid leukaemia. Leukemia \& Lymphoma, 7(5-6), 425-429.

Nakagawa, M., Koyanagi, M., Tanabe, K., et al. (2008). Generation of induced pluripotent stem cells without Myc from mouse and human fibroblasts. Nature Biotechnology, 26(1), 101-106.

Neff, A. W., King, M. W., \& Mescher, A. L. (2011). Dedifferentiation and the role of sall4 in reprogramming and patterning during amphibian limb regeneration. Developmental Dynamics, 240(5), 979-989.

Nielsen, J. S., \& McNagny, K. M. (2008). Novel functions of the CD34 family. Journal of Cell Science, 121(22), 3683-3692.

Norde, W. J., Hobo, W., van der Voort, R., et al. (2012). Coinhibitory molecules in hematologic malignancies: targets for therapeutic intervention. Blood, 120(4), 728-736.

Pabst, C., Krosl, J., Fares, I., et al. (2014). Identification of small molecules that support human leukemia stem cell activity ex vivo. Nature Methods, 11(4), 436-442.

Passegué, E., Jamieson, C. H., Ailles, L. E., et al. (2003). Normal and leukemic hematopoiesis: are leukemias a stem cell disorder or a reacquisition of stem cell 
characteristics? Proceedings of the National Academy of Sciences, 100 suppl 1, 1184211849.

Pearce, D. J., Taussig, D., Simpson, C., et al. (2005). Characterization of cells with a high aldehyde dehydrogenase activity from cord blood and acute myeloid leukemia samples. Stem Cells, 23(6), 752-760.

Porter, D. L., Levine, B. L., Kalos, M., et al. (2011). Chimeric antigen receptor-modified T cells in chronic lymphoid leukemia. New England Journal of Medicine, 365(8), 725-733.

Reikvam, H., Olsnes, A. M., Gjertsen, B. T., et al. (2009). Nuclear factor-kB signaling: a contributor in leukemogenesis and a target for pharmacological intervention in human acute myelogenous leukemia. Critical Reviews in Oncogenesis, 15(1-2), 1-41.

Reilly, J. T. (2005). Pathogenesis of acute myeloid leukaemia and inv (16)(p13; q22): a paradigm for understanding leukaemogenesis? British Journal of Haematology, 128(1), 18-34.

Salvatori, B., losue, I., Damas, N. D., et al. (2011). Critical role of c-myc in acute myeloid leukemia involving direct regulation of miR-26a and histone methyltransferase $\mathrm{EZH} 2$. Genes \& Cancer, 2(5), 585-592.

Sarry, J. E., Murphy, K., Perry, R., et al. (2011). Human acute myelogenous leukemia stem cells are rare and heterogeneous when assayed in NOD/SCID/IL2Ryc-deficient mice. The Journal of Clinical Investigation, 121(1), 384-395.

Schessl, C., Rawat, V. P., Cusan, M., et al. (2005). The AML1-ETO fusion gene and the FLT3 length mutation collaborate in inducing acute leukemia in mice. Journal of Clinical Investigation, 115(8), 2159-2168.

Shang, Y., Li, Z., Li, H., et al. (2013). TIM-3 expression in human osteosarcoma: Correlation with the expression of epithelial-mesenchymal transition-specific biomarkers. Oncology Letters, 6(2), 490-494.

Stella, C. C., Cazzola, M., De Fabritiis, P., et al. (1995). CD34-positive cells: biology and clinical relevance. Haematologica, 80(4), 367-387.

Sutherland, D. R., \& Keating A. (1992). The CD34 antigen: structure, biology, and potential clinical applications. Journal of Hematotherapy, 1(2), 115-129.

Suzuki, A., Andrew, D. P., Gonzalo, J. A., et al. (1996). CD34-deficient mice have reduced eosinophil accumulation after allergen exposure and show a novel crossreactive $90-k D$ protein. Blood, 87(9), 3550-3562. 
Swerdllow, S. H., Campo, E., \& Harris, N. L. (2008). WHO classification of tumours of haematopoietic and lymphoid tissues. France: IARC Press.

Takahashi, K., Tanabe, K., Ohnuki, M., et al. (2007). Induction of pluripotent stem cells from adult human fibroblasts by defined factors. Cell, 131(5), 861-872.

Tallman, M. S., Andersen, J. W., Schiffer, C. A., et al. (1997). All-trans-retinoic acid in acute promyelocytic leukemia. New England Journal of Medicine, 337(15), 1021-1028.

Tang, D., \& Lotze, M. T. (2012). Tumor immunity times out: TIM-3 and HMGB1.Nature Immunology, 13(9), 808-810.

Takahashi, K., \& Yamanaka, S. (2006). Induction of pluripotent stem cells from mouse embryonic and adult fibroblast cultures by defined factors. Cell, 126(4), 663-676.

Taussig, D. C., Miraki-Moud, F., Anjos-Afonso, F., et al. (2008). Anti-CD38 antibodymediated clearance of human repopulating cells masks the heterogeneity of leukemiainitiating cells. Blood, 112(3), 568-575.

Taussig, D. C., Vargaftig, J., Miraki-Moud, F., et al. (2010). Leukemia-initiating cells from some acute myeloid leukemia patients with mutated nucleophosmin reside in the CD34fraction. Blood, 115(10), 1976-1984.

Tavor, S., Eisenbach, M., Jacob-Hirsch, J., et al. (2008). The CXCR4 antagonist AMD3100 impairs survival of human AML cells and induces their differentiation. Leukemia, 22(12), 2151-5158.

Terpstra, W., Ploemacher, R. E., Prins, A., et al. (1996). Fluorouracil selectively spares acute myeloid leukemia cells with long-term growth abilities in immunodeficient mice and in culture. Blood, 88(6), 1944-1950.

Thomas, E. D. (1999). Bone marrow transplantation: a review. Seminars in Hematology, 36(4), 95-103.

Travis, M. A., \& Sheppard, D. (2014). TGF- $\beta$ Activation and Function in Immunity. Annual review of immunology, 32, 51-82.

van Stijn, A., van der Pol, M. A., Kok, A., et al. (2003). Differences between the CD34+ and CD34-blast compartments in apoptosis resistance in acute myeloid leukemia. Haematologica, 88(5), 497-508.

Watabe, T., \& Miyazono, K. (2009). Roles of TGF- $\beta$ family signaling in stem cell renewal and differentiation. Cell Research, 19(1), 103-115. 
Weiss, A., \& Attisano, L. (2013). The TGF- $\beta$ superfamily signaling pathway. Wiley Interdisciplinary Reviews: Developmental Biology, 2(1), 47-63.

Wiener, Z., Kohalmi, B., Pocza, P., et al. (2007). TIM-3 is expressed in melanoma cells and is upregulated in TGF- $\beta$ stimulated mast cells. Journal of Investigative Dermatology, 127(4), 906-914.

Wrzesinski, S. H., Wan, Y. Y., \& Flavell, R. A. (2007). Transforming growth factor- $\beta$ and the immune response: implications for anticancer therapy. Clinical Cancer Research, 13(18), 5262-5270.

Wu, M. Y., \& Hill, C. S. (2009). TGF- $\beta$ superfamily signaling in embryonic development and homeostasis. Developmental Cell, 16(3), 329-343.

Wu, F. H., Yuan, Y., Li, D., et al. (2010). Endothelial cell-expressed Tim-3 facilitates metastasis of melanoma cells by activating the NF-KB pathway. Oncology Reports, 24(3), 693-699.

Wulf, G. G., Wang, R. Y., Kuehnle, I., et al. (2001). A leukemic stem cell with intrinsic drug efflux capacity in acute myeloid leukemia. Blood, 98(4), 1166-1173.

Xu, J., Lamouille, S., \& Derynck, R. (2009). TGF- $\beta$-induced epithelial to mesenchymal transition. Cell Research, 19(2), 156-172.

Yoshimoto, G., Miyamoto, T., Jabbarzadeh-Tabrizi, S., et al. (2009). FLT3-ITD upregulates MCL-1 to promote survival of stem cells in acute myeloid leukemia via FLT3ITD-specific STAT5 activation. Blood, 114(24), 5034-5043.

Zamarron, B. F., \& Chen, W. (2011). Dual roles of immune cells and their factors in cancer development and progression. International Journal of Biological Sciences, 7(5), 651658.

Zhou, Q., Munger, M. E., Veenstra, R. G., et al. (2011). Coexpression of Tim-3 and PD-1 identifies a CD8+ T-cell exhaustion phenotype in mice with disseminated acute myelogenous leukemia. Blood, 117(17), 4501-4510.

Zhu, C., Anderson, A. C., Schubart, A., et al. (2005). The Tim-3 ligand galectin-9 negatively regulates T helper type 1 immunity. Nature Immunology, 6(12), 1245-1252. 


\title{
Chapter II
}

\author{
AML immune evasion and immune suppression; \\ therapeutic strategies for restoring immunologic function
}


When used alone, even chemotherapies that induce immunogenic tumor-cell death are unlikely to prevent relapse since the immune sytems of cancer patients are often so dysfunctional by the time treatment is initiated that even when they are stimulated by ICD they cannot eliminate immune-evasive and death-resistant CSCs. Thus, chemotherapies may need to be combined with patient-specific immune strategies that are specifically engineered to (1) eliminate poorly immunogenic CSCs, (2) repair and activate dysfunctional immune cells, and (3) neutralize immuno-suppressive pathways in tumor microenvironments. This chapter describes how malignant cells are able to resist immunologic attack by (1) growing rapidly, (2) avoiding recognition, (3) resisting death, and (4) suppressing anti-tumor defenses - see Figure 2.1 (Leone, 2012). Through immunoediting, the immune system selects for malignant-escape and antigen-loss variants that are poorly immunogenic (Dunn, 2002). As cancer cells evolve, they become poor stimulators - as well as poor targets - of immune cells (Whiteside, 2006). This chapter reviews strategies tumor cells employ to passively "hide" and actively escape from innate and adaptive immune cells (Igney, 2002). Of note, immune evasion has been more extensively studied in solid tumors than in blood cancers.

\section{Avoiding immune detection}

Tumor cells can avoid being recognized by the immune system (1) by migrating to immuno-privileged sites, (2) by altering their microenvironments in ways that reduce the survival, and impair the function, of immune cells, (3) by disrupting the presentation of tumor-cell antigens, and/or (4) by blocking costimulatory signals to antigen-presenting and immune-effector cells (Mapara, 2004; Whiteside, 2006; Mellman, 2011; Lion, 2012; Leone, 2012). Tumor cells can successfully grow in immuno-privileged sites such as the 
central nervous system and can create "metabolically hostile" microenvironments by altering local oxygen, $\mathrm{pH}$, and amino acid levels in ways that impair the physical entry, survival, and activity of infiltrating immune cells (Calcinotto, 2012; Baginska, 2013). Malignant cells can also induce the generation of physical and molecular barriers to infiltrating immune cells via the synthesis of dense extracellular matrix and/or expression of immuno-suppressive molecules like Tim-3 on tumor-associated vasculature (Huang, 2010) and/or galectins on surrounding stromal cells (Gieseke, 2010; Sioud, 2011).

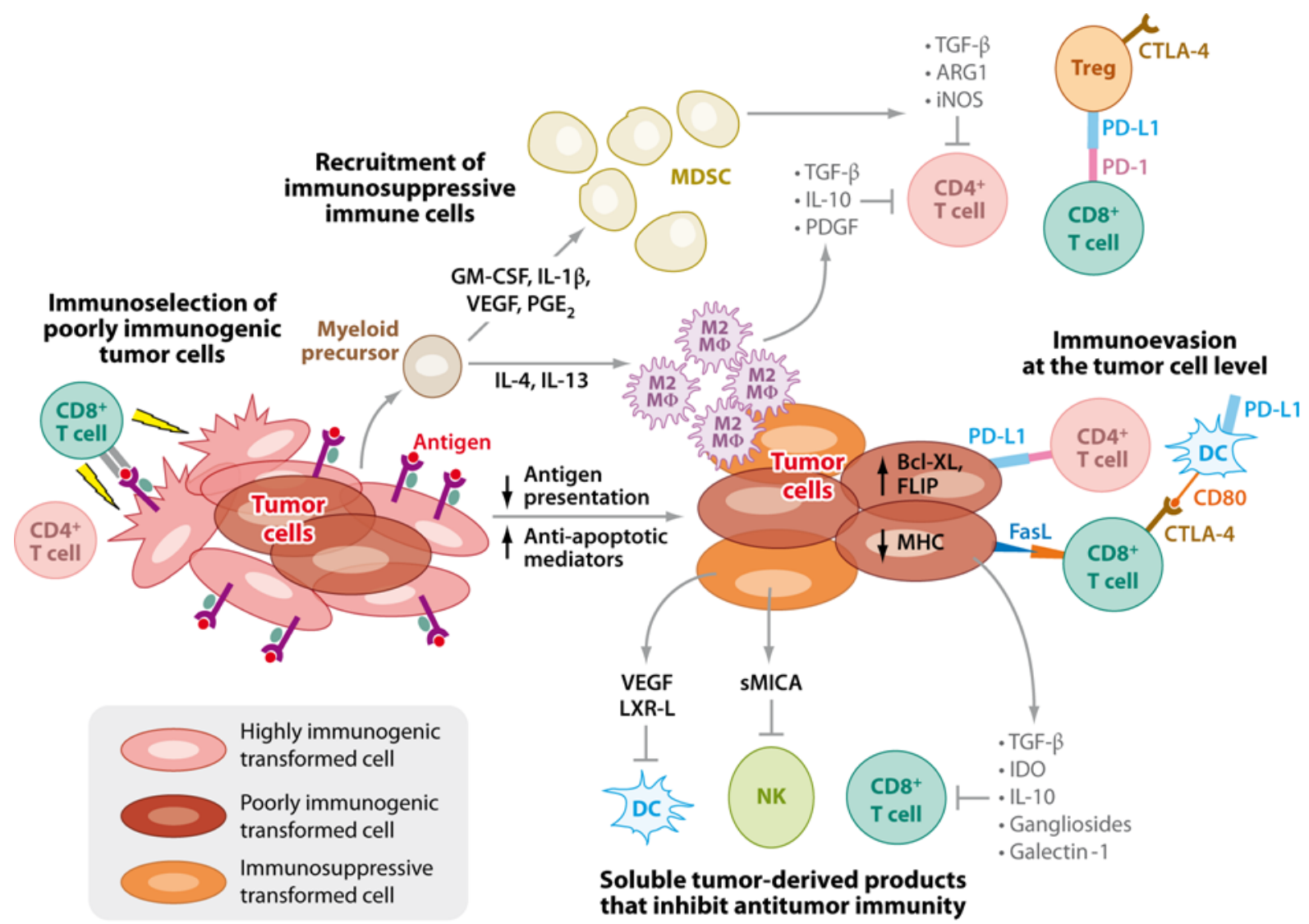

Figure 2.1. Tumor cell immune escape strategies. Tumor cells avoid immunemediated destruction through a variety of mechanisms, including poor antigen presentation and low immunogenicity, death resistance via high expression of antiapoptotic factors, expression of inhibitory receptors, release of immunosuppressive factors, and recruitment of immuno-suppressive cell types. Reproduced with permission from: Vesely, M. D., Kershaw, M. H., Schreiber, R. D., et al. (2011). Natural innate and adaptive immunity to cancer. Annual Review of Immunology, 29, 252 


\section{Impaired antigen-presentation}

Steps involved in the processing and presentation of tumor-cell antigens to the immune system via HLA class I molecules are reviewed in Figure 2.2. Normal- and tumorcell peptides are presented to T-helper $\left(T_{h}\right)$ cells via HLA class I molecules and to cytotoxic T cells via HLA class II molecules. Antigen-presenting cells (APCs), including DCs and monocytes/macrophages, normally present antigens to effector T cells via HLA class II molecules. Because myeloid leukemias arise from the same lineage as DCs and other APCs, AML cells are also able to present leukemic peptides to T cells on HLA class II complexes (van Luijn, 2010). Unfortunately, AML cells may behave like the immature DCs that promote immune tolerance (due to poor antigen-presentation) instead of functioning like the mature dendritic cells that promote immune clearance (Lutz, 2002; Panoskaltsis, 2005; Dudek, 2013). Although converting AML cells into DCs is being explored for the development of therapeutic cancer vaccines, caution must be taken: the differentiation of AML cells into indoleamine 2,3-diosygenase (IDO)-expressing DCs has been shown to impair maturation of normal DCs and inhibit T-cell proliferation, potentially restricting their use (Curti, 2010). 


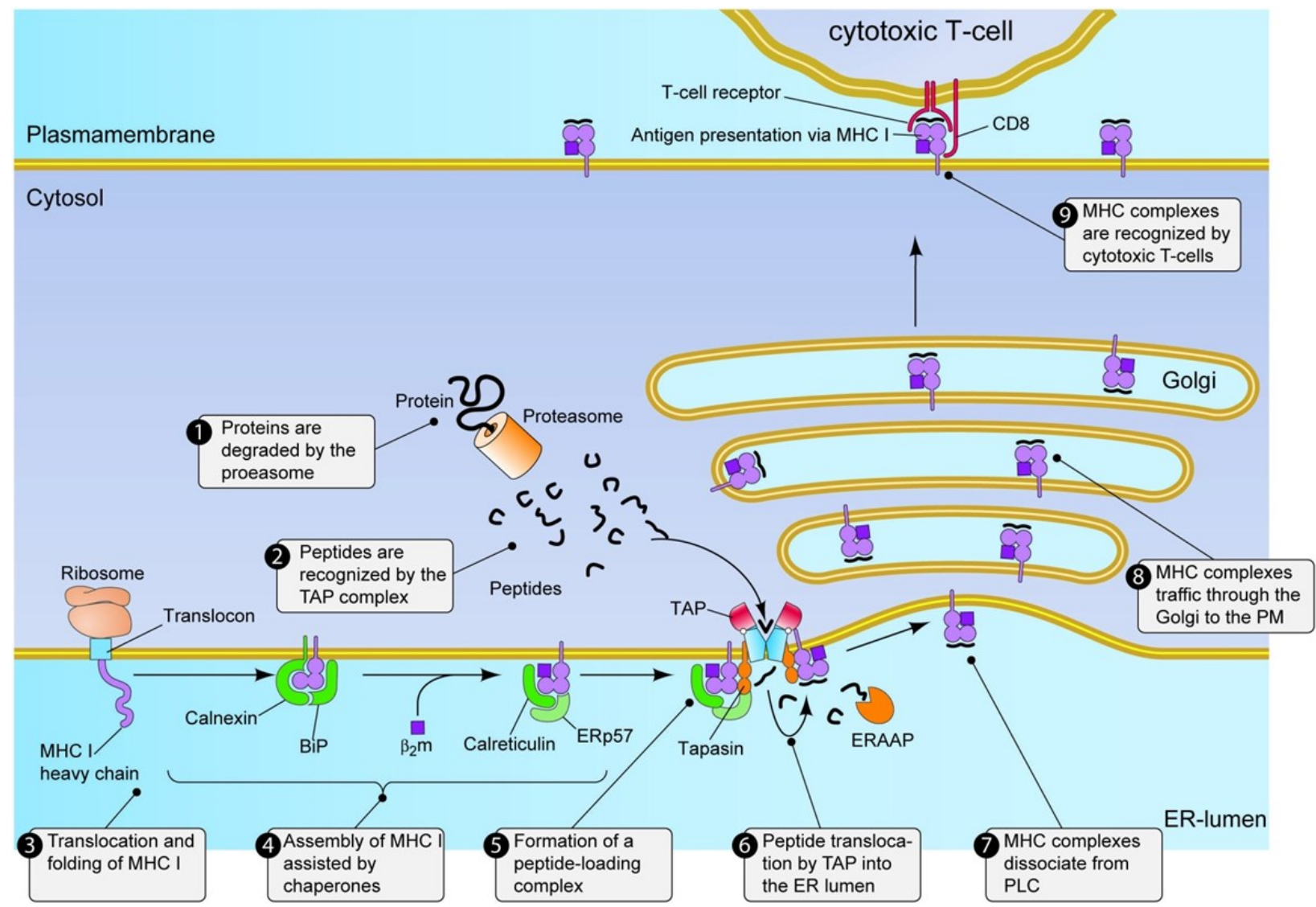

Figure 2.2. Processing and presentation of antigens via MHC class I molecules. Proteins are degraded within cells by the proteasome, loaded onto MHC class I molecules within the endoplasmic reticulum (ER), and translocated through the Golgi apparatus to the plasma membrane where peptide-MHC I complexes can be recognized by cytotoxic T cells. Reproduced with permission from: Parcej, D., \& Tampé, R. (2010). ABC proteins in antigen translocation and viral inhibition. Nature Chemical Biology, 6(8), 574. 
Regarding the ways AML cells can escape anti-tumor immunity, evolving AML cells may acquire the ability to disrupt the presentation and processing of tumor antigens, downregulate expression of immuno-stimulatory receptors and/or their ligands, and/or shed immune-receptor decoys (Hoves, 2009; Lion, 2012; Schmiedel, 2013; Elias, 2014). Evolving molecular changes can alter expression, and disrupt presentation, of tumor antigens which, in turn, can inhibit immune-cell activation (Leone, 2012). Like many other cancer cells, AML cells show reduced expression of HLA class 1 molecules compared to the non-malignant white blood cells (WBCs) from healthy donors which, in turn, impairs effective presentation of leukemia-associated antigens (Garrido, 2010; Vollmer, 2003; Vago, 2009). In addition, tumor-generated peptides may be poorly immunogenic or fail to fit in the HLA class 1 groove. AML peptide-HLA complexes may not be recognized by T cells or may be delivered to $\mathrm{T}$ cells in the absence of sufficient costimulatory signals (i.e. CD80 and CD86) which, in turn, promotes T-cell anergy, an unresponsive state (Vollmer, 2003). Persistence of class II-associated invariant chain peptide (CLIP) within HLA class II molecules impairs presentation of leukemic antigens while downregulation of CLIP enhances AML cell immunogenicity which, in turn, promotes activation of $\mathrm{Th}$ cells (van Luijn, 2010). Additional tumor-cell defects in antigen-processing machinery include mutations or deficiencies of proteasome subunits, including LMP2 and LMP7, and proteins that load peptides onto MHC class 1 molecules such as TAP and tapasin (Seliger, 1997; Johnsen, 1999; Leone, 2012).

\section{Promoting immune tolerance}

Immunologic tolerance is the inability to mount an immune response against an antigen. Central tolerance - aka "natural" or "self tolerance" which is normal and 
protective - is established during development when T cells that can attack normal, self antigens (and potentially trigger autoimmune diseases) are deleted in the thymus. Unfortunately, malignant cells generate a peripheral tolerance to tumor antigens by inducing T-cell anergy, by triggering T-cell apoptosis, and/or by deviating $\mathrm{T}_{\mathrm{h}} 1$ cytotoxic T-cell-mediated responses toward $\mathrm{T}_{\mathrm{h}} 2 \mathrm{~B}$-cell-mediated responses that are less effective against cancer cells (Mapara, 2004). Secretion of TGF- $\beta$ is one way tumor cells distort immune-cell responses by directing $\mathrm{Th}_{\mathrm{h}}$ cytotoxic $\mathrm{T}$-cell responses toward $\mathrm{Th}_{\mathrm{h}}$ humoral responses (Maeda, 1996). AML cells also recruit, or induce formation of, regulatory T cells ( $T_{\text {regs }}$ ) that suppress cytotoxic T cells. Through an IDO-dependent mechanism, AML cells promote conversion of CD4 ${ }^{+} \mathrm{CD} 25^{-} \mathrm{T}$ cells into $\mathrm{CD} 4^{+} \mathrm{CD} 25^{+} \mathrm{T}_{\text {regs }}$ (Curti, 2007). Finally, tumors can permanently delete tumor-reactive T-cell clones by expression of death-inducing ligands (Mapara, 2004) or by chronic (repetitive) antigen stimulation which triggers activation-induced cell death (Lu, 2008).

\section{Avoiding immune destruction}

\section{Resisting death}

Tumor cells resist immune-cell-mediated killing via the death receptor (Fasmediated) and/or granule exocytosis (perforin- and granzyme-mediated) pathways (Igney, 2002; Otten, 2004; Reed, 2003). Tumor cells overexpress anti-apoptotic factors (FLIP, Mcl-1, Bcl-2), resist pro-apoptotic signals, and display mutations or defects in apoptotic pathways (Chiu, 1995; Reed, 2003). Tumor cells also neutralize death-inducing stimuli by shedding soluble receptors that act as decoys for death ligands like TRAIL and FasL (Chamuleau, 2011; Reed, 2003). Strategies that can sensitize AML cells to T-cell or NK-cell killing include targeting anti-apoptotic factors that induce immune resistance. 
In a mouse model of colon cancer, treatment with a Nanog inhibitor decreased $\mathrm{Mcl}-1$ levels and sensitized tumor cells to T-cell-mediated lysis (Noh, 2012). Small-molecule inhibitors of Bcl-2, TRAIL-receptor agonists, and IAP antagonists are currently being evaluated as anti-cancer agents in clinical trials (Reed, 2003; Weyhenmeyer, 2012).

\section{Suppressing immune cells}

Malignant cells express soluble and membrane-bound immuno-suppressive factors that disable (by inducing defects) or eliminate cancer-cell-reactive immune cells (Whiteside, 2006; Gajewski, 2006). Immune cells can become dysfunctional when cancer cells (1) secrete soluble factors, (2) express immune-checkpoint receptors and/or their ligands, (3) recruit, and/or induce formation of, immuno-suppressive cells, and (4) distort stromal and immune cells within cancer-cell microenvironments (Huang, 2010; Gieseke, 2010; Mellman, 2011; Sioud, 2011). Immuno-suppressive factors can inhibit immune-cell activation, maturation, proliferation, survival, cytotoxicity, and proinflammatory cytokine secretion as well as recruit immuno-suppressive cells and unfavorably polarize immune responses (Spranger, 2013).

Cancer cells can express a variety of immuno-suppressive factors including TNFfamily ligands, immune-checkpoint receptors and their ligands (Kikushige, 2010), small molecules, enzymes, and cytokines (Whiteside, 2006; Vesely, 2011). Cancer-cell expression of TNF family ligands (such as FasL, TRAIL, TNF) triggers T-cell apoptosis upon binding to TNF family receptors (Whiteside, 2002). By increasing cAMP, small molecules like prostaglandin E2, histamine, and epinephrine inhibit anti-tumor immune responses by decreasing IL-2 and IFN-y production by $T$ cells as well as IL-1 $1 \beta, I L-12$, and TNFa production by monocytes and macrophages (Uotila, 1996). Expression of the 


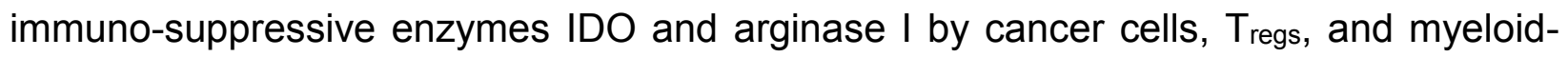
derived suppressor cells (MDSCs) impairs T-cell functions. By depleting tryptophan, IDO inhibits T-cell proliferation (Uyttenhove, 2003). Likewise, by depleting local L-arginine, arginase restricts T-cell proliferation and polarizes monocytes toward a suppressive M2-like phenotype (Mussai, 2013). Cancer-cell secretion of cytokines like TGF- $\beta$, IL-10, and GM-CSF inhibits the proliferation of $\mathrm{T}$ cells and the release of pro-inflammatory cytokines (Bergmann, 1995; Krüger-Krasagakes, 1994).

AML cells also directly inhibit T-cell activity by expressing coinhibitory receptors such as Tim-3 (Kikushige, 2010; Jan, 2011) and CTLA-4 (Laurent, 2007) as well as their ligands such as PD-1 ligand (Norde, 2011). By expressing higher levels of ligands for inhibitory NK-cell receptors than activating NK-cell receptors, AML cells impair NK-cellmediated lysis (Lion, 2012; Verheyden, 2008; Baessler, 2009; Baessler, 2010). AML expression of CD200 and RANKL inhibits NK-cell activity (Coles, 2011; Schmiedel, 2013) and T-cell activity (Coles, 2012). AML cells also escape NK-cell-mediated killing by secreting MICA and MICB ligands as well as by shedding NKG2D-receptor decoys that absorb activating NK-cell ligands (Weiss-Steider, 2011; Groh, 2002).

Therapies that target immune-checkpoint receptors and immuno-suppressive factors in the tumor microenvironment are reviewed in Chapter IV. In addition to tumorcell-derived and immune-cell-derived factors, CAFs and endothelial cells within the tumor vasculature may also play an important role in immune suppression (Huang, 2010; Gieseke, 2010; Sioud, 2011). Thus, for some cancer patients, effective multi-pronged therapies may need to systemically modulate the tumor-sustaining support cells in addition to targeting the tumor cells. 


\section{Strategies to enhance tumor-cell immunogenicity}

It is believed most cancers do, initially, trigger adaptive immune responses evidenced by the presence of circulating tumor-reactive antibodies and immune cells. Over time, however, immunogenic cancer cells are eliminated, favoring the outgrowth of immune-escape variants (Dunn, 2002). When considering how to target AML cells, possible immunologic strategies include increasing AML-cell immunogenicity and/or rendering AML cells less death-resistant (more susceptible to immunologic attack). Increasing the immunogenicity of AML LSCs may be important for eradicating therapyresistant "minimal residual disease" cells that can trigger relapse (Gerber, 2012). AML LSCs are problematic because they are especially death-resistant and are better able to escape immune recognition and destruction than more mature leukemic blasts (Costello, 2000). As expected, poorly differentiated AML subtypes (M0) have been found to be less immunogenic than the more differentiated subtypes (M4-M5) as evidenced by weak in vitro alloimmune recognition of the poorly differentiated AML cells by $T$ cells and their

reduced expression of costimulatory molecules (Costello, 1999). In general, CSCs only weakly stimulate immune cells; thus ex vivo activation of a patient's immune cells may require manipulation of stem-like cancer cells to improve their immunogenicity. Potential strategies to increase AML-cell immunogenicity - the ability to stimulate an adaptive immune response - include enhancing antigen-presentation by leukemia cells, blocking receptors like the "do not eat me" signals involved in immune escape, and inducing immunogenic tumor-cell death (Majeti, 2009; Lion, 2011; Rosenblatt, 2012). 


\section{Improving antigen-presentation by leukemia cells}

AML cells can directly activate, and present antigens to, immune cells in vitro - at least y9ס2 T-cells (Gertner-Dardenne, 2012). That is, antigen-presentation and immunecell activation by AML cells can occur simultaneously. Perhaps AML-cell immunogenicity and antigen-presentation are best envisioned in the context of the immunologic synapse between AML and immune cells. Of note, in co-culture systems, TCR agonists clearly enhance the direct activation of $\mathrm{T}$ cells by AML cells (Gertner-Dardenne, 2012). Presumably, a variety of other factors (cytokines, antibodies, immuno-stimulatory compounds, and agents known to modulate antigen-presentation pathways, etc.) can also enhance in vitro immune-cell activation by improving antigen-presentation, immunecell function, and/or tumor-cell immunogenicity.

Of note, cytokine-induced differentiation of leukemia cells into DCs has been shown to improve the presentation of leukemia cell antigens to T cells (Cignetti, 1999; Charbonier, 1999; Harrison, 2001; Woiciechowsky, 2001; Choudhury, 1999). Exposing AML cells to the TLR 7/8 agonist resiquimod, for example, enhanced their expression of MHC molecules, production of pro-inflammatory cytokines, and ability to stimulate allogeneic T cells (Smits, 2010). Silencing STAT3 in AML cells upregulated HLA class II molecules along with costimulatory and proinflammatory mediators (like IL-12) while decreasing expression of the coinhibitory molecule PD-L1 and improving activation of $\mathrm{CD}^{+} \mathrm{T}$ cells (Hossain, 2014). Stimulation with CD40 and IFN- $\gamma$ increased AML immunogenicity and expression of costimulatory molecules (Costello, 1999). Exposure to the histone deacetylase inhibitor (HDAC) chidamide increased AML expression of the cancer testis antigen PRAME and susceptibility to T-cell-mediated cytotoxicity (Yao, 
2013). In addition, inhibiting nonsense-mediated messenger RNA decay improved the immunogenicity of cancer cells by inducing expression of novel immunogenic tumor antigens (Pastor, 2010). Proteasome inhibitors like bortezomib may also improve the immunogenicity of malignant cells by inducing expression of HSP90, a "danger signal," on the surface of myeloma cells (Gavioli, 2002).

\section{Genetic modification of leukemia cells}

To increase their ability to activate immune cells, AML cells can be engineered to express danger signals, costimulatory molecules, or proinflammatory compounds (Lion, 2011). Transfection of AML cells with the viral mimetic polyinosinic polycytidylic acid [poly(l:C)], a synthetic non-coding synthetic dsRNA analog, enhanced DC activation and increased IFN-y secretion by natural killer (NK) cells (Smits, 2007). Following transfection with poly(l:C), AML cells were also more susceptible to killing by NK cells and phagocytosis by DCs, suggesting this dsRNA analog may be a useful vaccine adjuvant (Lion, 2011). Downregulating CLIP expression by AML blasts increased their ability to stimulate CD4 ${ }^{+}$T-cell proliferation (van Luijn, 2010) consistent with the enhanced ability of CLIP(-) compared to CLIP(+) AML cells to activate and polarize CD4 ${ }^{+} \mathrm{T}$ cells toward Th1 cells (van Luijn, 2011). Modifying AML cells to express the costimulatory ligand CD80 also enhanced their ability to stimulate T-cell proliferation (Mutis, 1998).

\section{Inducing immunogenic tumor-cell death}

Cytotoxic chemotherapies are generally believed to suppress the immune system through myelotoxicity. However, radiotherapy and certain chemotherapeutic drugs induce immunogenic forms of tumor-cell death characterized by cell-surface changes that promote the phagocytosis of dying tumor cells and the release of immuno-stimulating 
compounds - see Figure 2.3 (Tesniere, 2008; Inoue, 2014). Ionizing radiation $(\mathrm{\gamma}$-irradiation) - as well as chemotherapies that include anthracyclines (daunorubicin, idarubicin, mitoxanthrone), the platinum derivate oxaliplatin, and the alkylating agent cyclophosphamide - induce immunogenic tumor-cell death (Reits, 2006; Garnett, 2004; Casares, 2005; Obeid, 2007; Panaretakis, 2008). Therapies that induce immunogenic cell death (ICD) can serve as in situ "cryptic vaccines" via release of tumor antigens and immune-boosting danger signals (Ma, 2010). After reviewing the chronic lymphocytic leukemia (CLL) trials that exploited adoptive transfer of autologous CAR-modified T cells, Dr. June speculated that pre-treatment of patients with immunogenic chemotherapies may have improved therapeutic outcomes (Kalos, 2011; Porter, 2011). In addition to killing tumor cells, cytotoxic chemotherapies can enhance immune responses by inducing transient lymphodepletion, counteracting immuno-suppressive mechanisms (Lutsiak, 2005), stimulating immune cells, and sensitizing tumor cells to T-cell or NK-cell-mediated killing (Ma, 2010). ICD might explain the "abscopal effect" in which localized radiation can also shrink metastatic tumors far from irradiated areas - presumably by stimulating the immune system (Kingsley, 1975; Robin, 1981; Wersall, 2006; Ohba, 1998).

Unfortunately for cancer research, the immunological effects of anti-cancer drugs cannot be evaluated in immuno-deficient mice transplanted with human tumors. One method to evaluate ICD involves pre-treatment of tumor cells with various chemotherapeutic drugs or cell-damaging agents followed by their subcutaneous injection into syngeneic immuno-competent mice. When inoculated with tumor cells undergoing ICD, mice generate anti-tumor immune responses and establish long-term immunologic memory which prevents tumor growth upon subsequent challenge with live 
tumor cells. In vitro immune-cell activation assays that measure proliferation, cytokine release, and cytotoxicity are also available.

Features of immunogenic cell death (ICD)

During ICD, tumor-cell antigens are released along with pro-inflammatory stimuli such as "find me," "eat me," and "danger" signals that stimulate anti-tumor immunity - see Figure 2.3. The molecular characteristics of ICD have been nicely reviewed (Tesniere, 2008; Kepp, 2011). In general, pro-inflammatory "danger" signals promote tumor-antigen uptake, processing, and presentation - as well as enhance the anti-tumor activity of immune cells. Inducing the ICD of malignant cells is one strategy that can promote "immunogenic conversion" of the tumor microenvironment - see Figure 2.4. Current conceptual frameworks for immunology suggest it is worthwhile to "convert" cancer cells into a "cryptic vaccine" by inducing release of tumor antigens in an immuno-stimulatory context. 


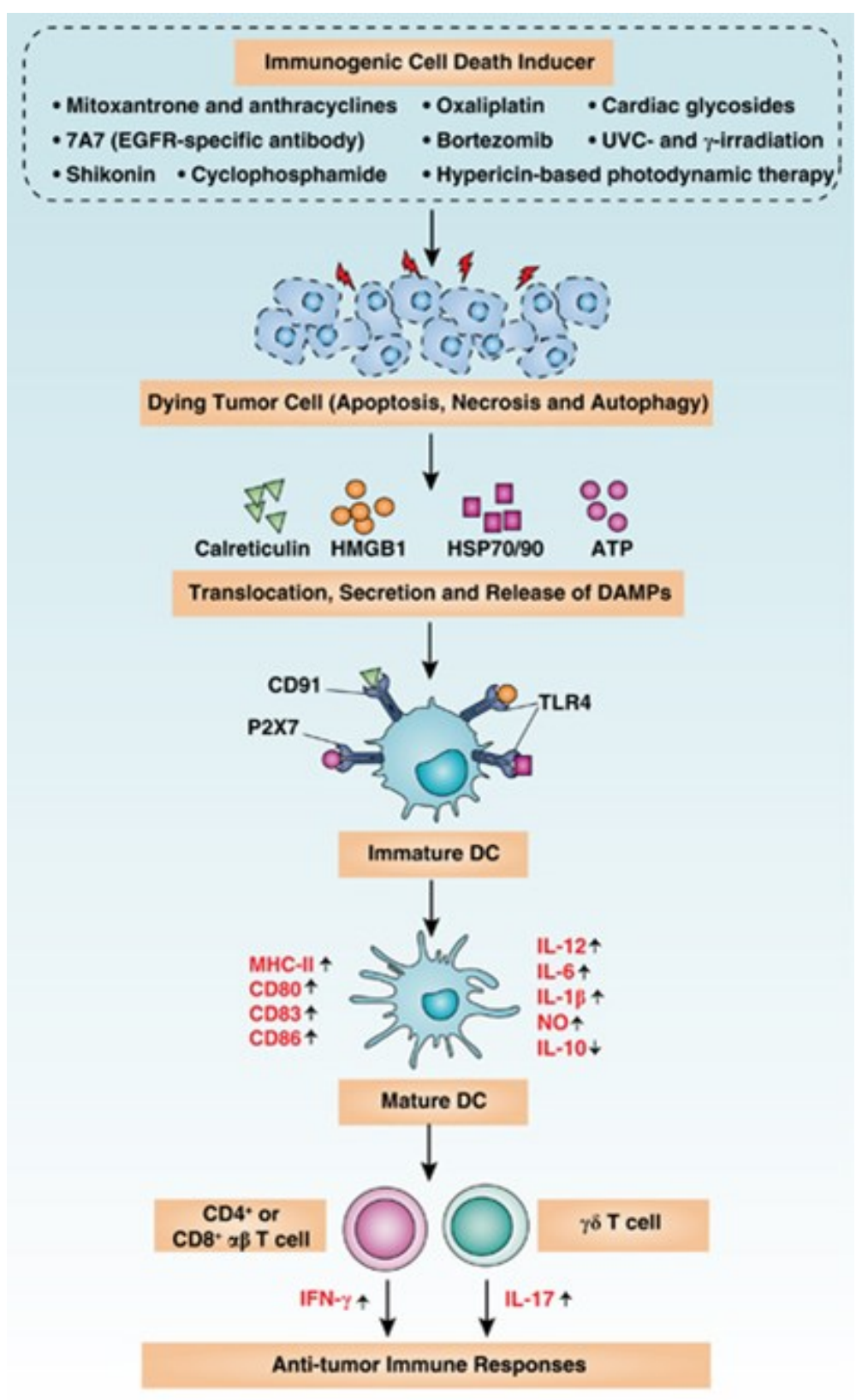

Figure 2.3. Features of immunogenic tumor cell death. A variety of chemotherapeutic drugs, radiotherapy, and targeted anti-cancer agents induce immunogenic forms of tumor cell death. During immunogenic cell death, tumor cells expose and release a variety of danger signals. For example, heat shock proteins (HSP) and calreticulin translocate to the plasma membrane and ATP and HMGB1 are secreted. In response to these danger signals, DCs mature into effective antigen-presenting cells that prime $T$ cells to attack tumors. Reproduced with permission from: Hou, W., Zhang, Q., Yan, Z., et al. (2013). Strange attractors: DAMPs and autophagy link tumor cell death and immunity. Cell Death \& Disease, 4(12), 5. 


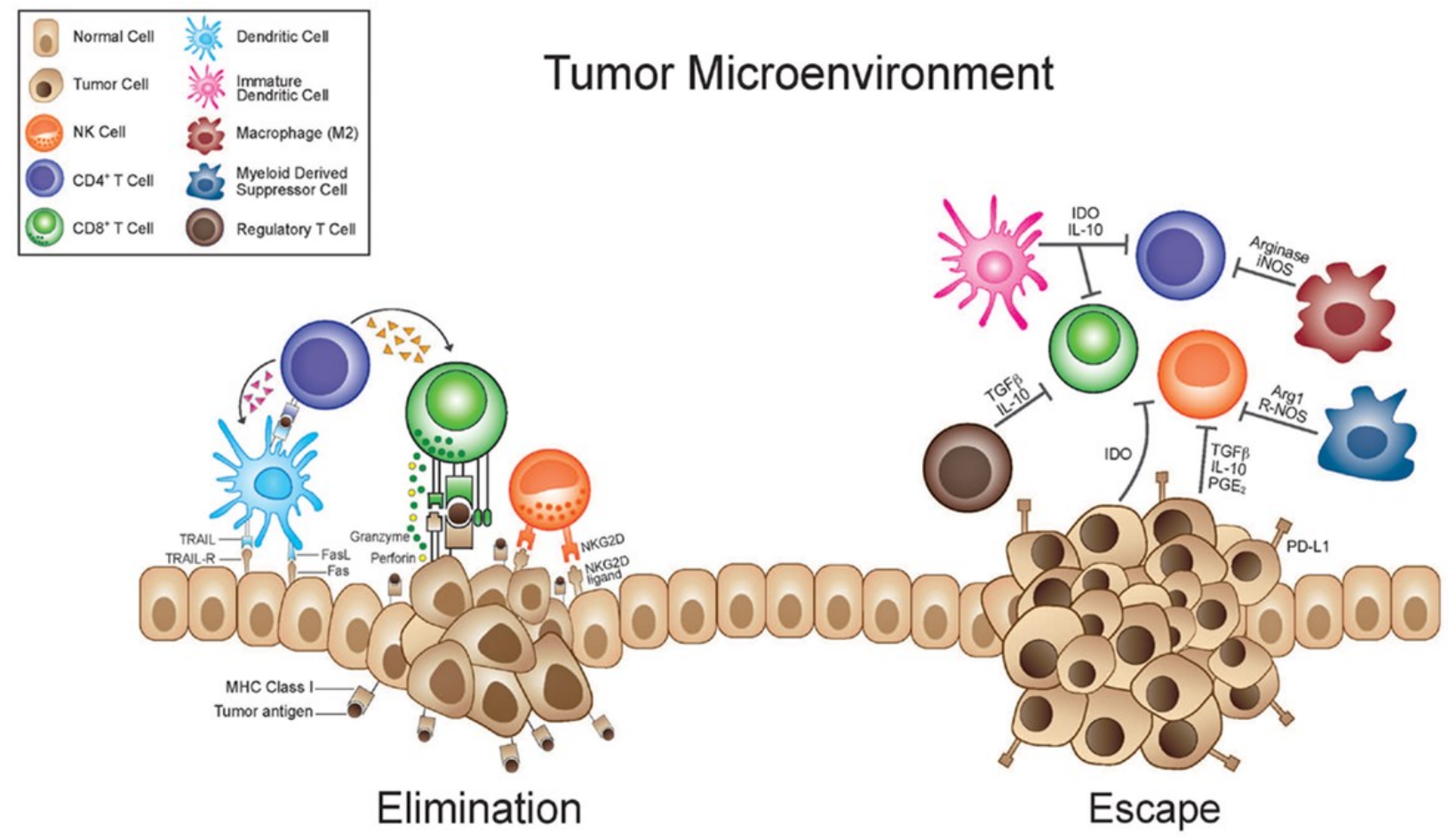

Figure 2.4. Comparison of immunosupportive and immunosuppressive tumor microenvironments. Within immunogenic microenvironments, activated immune cells recognize and eliminate tumor cells. In contrast, within non-immunogenic microenvironments, tumor cells release immunosuppressive factors (such as TGF- $\beta$ and IL-10) and recruit $T_{\text {reg }}$ cells and MDSCs that subvert infiltrating antigen-presenting and immune-effector cells; this leads to immune escape and tumor progression. A major goal of cancer immunotherapy is to convert immunosuppressive tumor microenvironments that promote tumor escape into immunosupportive tissues that eradicate malignancy. Reproduced with permission from: Monjazeb, A. M., Zamora, A. E., Grossenbacher, S. $\mathrm{K}$., et al. (2013). Immunoediting and antigen loss: overcoming the Achilles heel of immunotherapy with antigen non-specific therapies. Frontiers in Oncology, 3, 4. 
"Find me" signals include small molecules such as HMGB1 and ATP that help recruit and activate infiltrating immune cells like monocytes. HMGB1 binds TLR4 on DCs and inhibits antigen degradation mediated by fusion of phagosomes with lysosomes, thereby favoring the processing and presentation of tumor antigens (Apetoh, 2007). ATP released from cancer cells binds $\mathrm{P} 2 \mathrm{RX} 7$ on DCs, leading to inflammasome activation and secretion of IL-1 $\beta$ (Ghiringhelli, 2009). "Eat me" signals stimulate uptake of tumor antigens by antigen-presenting cells like DCs. During ICD, malignant cells express surface calreticulin. When translocated to the plasma membrane, calreticulin functions as a receptor for DC engulfment (Obeid, 2007). During ICD, cancer cells may also downregulate expression of "do not eat me" signals such as CD47. Tumor-cell loss or the redistribution of CD47, which normally inhibits phagocytosis by SIRPa-expressing macrophages, can promote cancer-cell engulfment due to expression of calreticulin (Willingham, 2012). In response to the cellular stress induced by chemotherapy, cancer cells transcriptionally activate heat-shock proteins which, in turn, stimulate DC maturation via CD91 (Somersan, 2001) and facilitate chaperoning of tumor antigens to MHC molecules for presentation to T cells (Zitvogel, 2008; Binder, 2005).

While this chapter focuses on factors mediating immune evasion and suppression on the tumor-cell side of malignant disease, Chapter III focuses on the cancer-induced defects of immune cells and the tumor stroma that prevent immune clearance of cancer cells. Strategies are reviewed for (1) the repair of dysfunctional immune cells (in vitro) followed by their activation and expansion and (2) the reversal of the immune suppression induced by cancer microenvironments (in vivo). The ways Tim-3 expression can impair 
anti-tumor immune responses - when expressed on the surface of tumor or immune cells

- are also described.

\section{References}

Apetoh, L., Ghiringhelli, F., Tesniere, A., et al. (2007). Toll-like receptor 4-dependent contribution of the immune system to anticancer chemotherapy and radiotherapy. Nature Medicine, 13(9), 1050-1059.

Baessler, T., Charton, J. E., Schmiedel, B. J., et al. (2010). CD137 ligand mediates opposite effects in human and mouse NK cells and impairs NK-cell reactivity against human acute myeloid leukemia cells. Blood, 115(15), 3058-3069.

Baessler, T., Krusch, M., Schmiedel, B. J., et al. (2009). Glucocorticoid-Induced Tumor Necrosis Factor Receptor-Related Protein Ligand Subverts Immunosurveillance of Acute Myeloid Leukemia in Humans. Cancer Research, 69(3), 1037-1045.

Baginska, J., Viry, E., Paggetti, J., et al. (2013). The critical role of the tumor microenvironment in shaping natural killer cell-mediated anti-tumor immunity. Frontiers inlimmunology, 4, 490.

Bergmann, L., Schui, D. K., Brieger, J., et al. (1995). The inhibition of lymphokineactivated killer cells in acute myeloblastic leukemia is mediated by transforming growth factor-beta 1. Experimental Hematology, 23(14), 1574-1580.

Binder, R. J., \& Srivastava, P. K. (2005). Peptides chaperoned by heat-shock proteins are a necessary and sufficient source of antigen in the cross-priming of CD8+ $T$ cells. Nature Immunology, 6(6), 593-599.

Blankenstein, T., Coulie, P. G., Gilboa, E., et al. (2012). The determinants of tumour immunogenicity. Nature Reviews Cancer, 12(4), 307-313.

Calcinotto, A., Filipazzi, P., Grioni, M., et al. (2012). Modulation of microenvironment acidity reverses anergy in human and murine tumor-infiltrating $T$ lymphocytes. Cancer Research, 72(11), 2746-2756.

Casares, N., Pequignot, M. O., Tesniere, A., et al. (2005). Caspase-dependent immunogenicity of doxorubicin-induced tumor cell death. The Journal of Experimental Medicine,202(12), 1691-1701.

Chamuleau, M. E. D., Ossenkoppele, G. J., Van Rhenen, A., et al. (2011). High TRAILR3 expression on leukemic blasts is associated with poor outcome and induces 
apoptosis-resistance which can be overcome by targeting TRAIL-R2. Leukemia Research, 35(6), 741-749.

Charbonnier, A., Gaugler, B., Sainty, D., et al. (1999). Human acute myeloblastic leukemia cells differentiate in vitro into mature dendritic cells and induce the differentiation of cytotoxic T cells against autologous leukemias. European Journal of Immunology, 29(8), 2567-2578.

Chiu, V. K., Walsh, C. M., Liu, C. C., et al. (1995). Bcl-2 blocks degranulation but not fasbased cell-mediated cytotoxicity. The Journal of Immunology, 154(5), 2023-2032.

Choudhury, A., Liang, J. C., Thomas, E. K., et al. (1999). Dendritic cells derived in vitro from acute myelogenous leukemia cells stimulate autologous, antileukemic T-cell responses. Blood, 93(3), 780-786.

Cignetti, A., Bryant, E., Allione, B., et al. (1999). CD34+ acute myeloid and lymphoid leukemic blasts can be induced to differentiate into dendritic cells. Blood, 94(6), 20482055.

Coles, S. J., Hills, R. K., Wang, E. C. Y., et al. (2012). Expression of CD200 on AML blasts directly suppresses memory T-cell function. Leukemia, 26(9), 2148.

Coles, S. J., Wang, E. C. Y., Man, S., et al. (2011). CD200 expression suppresses natural killer cell function and directly inhibits patient anti-tumor response in acute myeloid leukemia. Leukemia, 25(5), 792-799.

Costello, R. T., Mallet, F., Chambost, H., et al. (1999). The immunophenotype of minimally differentiated acute myeloid leukemia (AML-M0): reduced immunogenicity and high frequency of CD34+/CD38-leukemic progenitors. Leukemia, 13(10), 1513-1518.

Costello, R. T., Mallet, F., Gaugler, B., et al. (2000). Human acute myeloid leukemia CD34+/CD38- progenitor cells have decreased sensitivity to chemotherapy and Fasinduced apoptosis, reduced immunogenicity, and impaired dendritic cell transformation capacities. Cancer Research, 60(16), 4403-4411.

Curti, A., Pandolfi, S., Valzasina, B., et al. (2007). Modulation of tryptophan catabolism by human leukemic cells results in the conversion of CD25- into CD25+ T regulatory cells. Blood,109(7), 2871-2877.

Curti, A., Trabanelli, S., Onofri, C., et al. (2010). Indoleamine 2, 3-dioxygenaseexpressing leukemic dendritic cells impair a leukemia-specific immune response by inducing potent T regulatory cells. Haematologica, 95(12), 2022-2030.

Dudek, A. M., Martin, S., Garg, A. D., et al. (2013). Immature, semi-mature, and fully mature dendritic cells: toward a DC-cancer cells interface that augments anticancer immunity. Frontiers in Immunology, 4(438), 1-14. 
Dunn, G. P., Bruce, A. T., Ikeda, H., et al. (2002). Cancer immunoediting: from immunosurveillance to tumor escape. Nature Immunology, 3(11), 991-998.

Elias, S., Yamin, R., Golomb, L., et al. (2014). Immune evasion by oncogenic proteins of acute myeloid leukemia. Blood, 123(10), 1535-1543.

Gajewski, T. F., Meng, Y., Blank, C., et al. (2006). Immune resistance orchestrated by the tumor microenvironment. Immunological Reviews, 213(1), 131-145.

Garnett, C. T., Palena, C., Chakarborty, M., et al. (2004). Sublethal irradiation of human tumor cells modulates phenotype resulting in enhanced killing by cytotoxic $T$ lymphocytes. Cancer Research, 64(21), 7985-7994.

Garrido, F., Cabrera, T., \& Aptsiauri, N. (2010). "Hard" and "soft" lesions underlying the HLA class I alterations in cancer cells: implications for immunotherapy. International Journal of Cancer, 127(2), 249-256.

Gerber, J. M., Smith, B. D., Ngwang, B., et al. (2012). A clinically relevant population of leukemic CD34+ CD38- cells in acute myeloid leukemia. Blood, 119(15), 3571-3577.

Ghiringhelli, F., Apetoh, L., Tesniere, A., et al. (2009). Activation of the NLRP3 inflammasome in dendritic cells induces IL-1 $\beta$-dependent adaptive immunity against tumors. Nature Medicine,15(10), 1170-1178.

Gieseke, F., Kruchen, A., Tzaribachev, N., et al. (2013). Proinflammatory stimuli induce galectin-9 in human mesenchymal stromal cells to suppress T-cell proliferation. European Journal of Immunology, 43(10), 2741-2749.

Groh, V., Wu, J., Yee, C., et al. (2002). Tumour-derived soluble MIC ligands impair expression of NKG2D and T-cell activation. Nature, 419(6908), 734-738.

Harrison, B. D., Adams, J. A., Briggs, M., et al. (2001). Stimulation of autologous proliferative and cytotoxic T-cell responses by "leukemic dendritic cells" derived from blast cells in acute myeloid leukemia. Blood, 97(9), 2764-2771.

Hossain, D. M. S., Dos Santos, C., Zhang, Q., et al. (2014). Leukemia cell-targeted STAT3 silencing and TLR9 triggering generate systemic antitumor immunity. Blood, 123(1), 15-25.

Hou, W., Zhang, Q., Yan, Z., et al. (2013). Strange attractors: DAMPs and autophagy link tumor cell death and immunity. Cell Death \& Disease, 4(12), 1-10.

Hoves, S., Aigner, M., Pfeiffer, C., et al. (2009). In situ analysis of the antigen-processing machinery in acute myeloid leukaemic blasts by tissue microarray. Leukemia, 23(5), 877885. 
Huang, X., Bai, X., Cao, Y., et al. (2010). Lymphoma endothelium preferentially expresses Tim-3 and facilitates the progression of lymphoma by mediating immune evasion. The Journal of Experimental Medicine, 207(3), 505-520.

Igney, F. H., \& Krammer, P. H. (2002). Immune escape of tumors: apoptosis resistance and tumor counterattack. Journal of Leukocyte Biology, 71(6), 907-920.

Inoue, H., \& Tani, K. (2014). Multimodal immunogenic cancer cell death as a consequence of anticancer cytotoxic treatments. Cell Death \& Differentiation, 21(1), 3949.

Jajosky, A. N., Coad, J. E., Vos, J. A., et al. (2014). RepSox slows decay of CD34+ acute myeloid leukemia cells and decreases $T$ cell immunoglobulin mucin-3 expression. Stem Cells Translational Medicine, 3(7), 836-848.

Jan, M., Chao, M. P., Cha, A. C., et al. (2011). Prospective separation of normal and leukemic stem cells based on differential expression of TIM3, a human acute myeloid leukemia stem cell marker. Proceedings of the National Academy of Sciences, 108(12), 5009-5014.

Johnsen, A. K., Templeton, D. J., Sy, M. S., et al. (1999). Deficiency of transporter for antigen presentation (TAP) in tumor cells allows evasion of immune surveillance and increases tumorigenesis. The Journal of Immunology, 163(8), 4224-4231.

Kalos, M., Levine, B. L., Porter, D. L., et al. (2011). T cells with chimeric antigen receptors have potent antitumor effects and can establish memory in patients with advanced leukemia. Science Translational Medicine, 3(95), 95ra73.

Kepp, O., Galluzzi, L., Martins, I., et al. (2011). Molecular determinants of immunogenic cell death elicited by anticancer chemotherapy. Cancer and Metastasis Reviews, 30(1), 61-69.

Kikushige, Y., Shima, T., Takayanagi, S. I., et al. (2010). TIM-3 is a promising target to selectively kill acute myeloid leukemia stem cells. Cell Stem Cell, 7(6), 708-717.

Kingsley, D. P. E. (1975). An interesting case of possible abscopal effect in malignant melanoma. The British Journal of Radiology, 48(574), 863-866.

Krüger-Krasagakes, S., Krasagakis, K., Garbe, C., et al. (1994). Expression of interleukin 10 in human melanoma. British Journal of Cancer, 70(6), 1182-1185.

Laurent, S., Palmisano, G. L., Martelli, A. M., et al. (2007). CTLA-4 expressed by chemoresistant, as well as untreated, myeloid leukaemia cells can be targeted with ligands to induce apoptosis. British Journal of Haematology, 136(4), 597-608. 
Leone, P., Shin, E. C., Perosa, F., et al. (2013). MHC class I antigen processing and presenting machinery: organization, function, and defects in tumor cells. Journal of the National Cancer Institute, 105(16), 1172-1187.

Lion, E., Anguille, S., Berneman, Z. N., et al. (2011). Poly (I: C) enhances the susceptibility of leukemic cells to NK cell cytotoxicity and phagocytosis by DC. PloS One, 6(6), e20952, 1-10.

Lion, E., Smits, E. L., Berneman, Z. N., et al. (2009). Acute myeloid leukemic cell lines loaded with synthetic dsRNA trigger IFN- $\gamma$ secretion by human NK cells. Leukemia Research, 33(4), 539-546.

Lion, E., Willemen, Y., Berneman, Z. N., et al. (2012). Natural killer cell immune escape in acute myeloid leukemia. Leukemia, 26(9), 2019-2026.

Lu, B., \& Finn, O. J. (2008). T-cell death and cancer immune tolerance. Cell Death \& Differentiation, 15(1), 70-79.

Lutsiak, M. C., Semnani, R. T., De Pascalis, R., et al. (2005). Inhibition of CD4+ 25+ T regulatory cell function implicated in enhanced immune response by low-dose cyclophosphamide. Blood, 105(7), 2862-2868.

Lutz, M. B., \& Schuler, G. (2002). Immature, semi-mature and fully mature dendritic cells: which signals induce tolerance or immunity? Trends in Immunology, 23(9), 445-449.

Ma, Y., Kepp, O., Ghiringhelli, F., et al. (2010). Chemotherapy and radiotherapy: cryptic anticancer vaccines. Seminars in Immunology, 22(3), 113-124.

Maeda, H., \& Shiraishi, A. (1996). TGF-beta contributes to the shift toward Th2-type responses through direct and IL-10-mediated pathways in tumor-bearing mice. The Journal of Immunology, 156(1), 73-78.

Majeti, R., Chao, M. P., Alizadeh, A. A., et al. (2009). CD47 is an adverse prognostic factor and therapeutic antibody target on human acute myeloid leukemia stem cells. Cell, 138(2), 286-299.

Mapara, M. Y., \& Sykes, M. (2004). Tolerance and cancer: mechanisms of tumor evasion and strategies for breaking tolerance. Journal of Clinical Oncology, 22(6), 1136-1151.

Mellman, I., Coukos, G., \& Dranoff, G. (2011). Cancer immunotherapy comes of age. Nature, 480(7378), 480-489.

Mussai, F., De Santo, C., Abu-Dayyeh, I., et al. (2013). Acute myeloid leukemia creates an arginase-dependent immunosuppressive microenvironment. Blood, 122(5), 749-758. 
Mutis, T., Schrama, E., Melief, C. J., et al. (1998). CD80-transfected acute myeloid leukemia cells induce primary allogeneic T-cell responses directed at patient specific minor histocompatibility antigens and leukemia-associated antigens. Blood, 92(5), 16771684.

Noh, K. H., Kim, B. W., Song, K. H., et al. (2012). Nanog signaling in cancer promotes stem-like phenotype and immune evasion. The Journal of Clinical Investigation, 122(11), 4077-4093.

Norde, W. J., Maas, F., Hobo, W., et al. (2011). PD-1/PD-L1 interactions contribute to functional T-cell impairment in patients who relapse with cancer after allogeneic stem cell transplantation. Cancer research, 71(15), 5111-5122.

Obeid, M., Tesniere, A., Ghiringhelli, F., et al. (2007). Calreticulin exposure dictates the immunogenicity of cancer cell death. Nature Medicine, 13(1), 54-61.

Ohba, K., Omagari, K., Nakamura, T., et al. (1998). Abscopal regression of hepatocellular carcinoma after radiotherapy for bone metastasis. Gut, 43(4), 575-577.

Otten, H. G., Van Ginkel, W. G. J., Hagenbeek, A., et al. (2004). Prevalence and clinical significance of resistance to perforin-and FAS-mediated cell death in leukemia. Leukemia, 18(8), 1401-1405.

Panaretakis, T., Joza, N., Modjtahedi, N., et al. (2008). The co-translocation of ERp57 and calreticulin determines the immunogenicity of cell death. Cell Death \& Differentiation, 15(9), 1499-1509.

Panoskaltsis, N. (2005). Dendritic cells in MDS and AML-cause, effect or solution to the immune pathogenesis of disease? Leukemia, 19(3), 354-357.

Parcej, D., \& Tampé, R. (2010). ABC proteins in antigen translocation and viral inhibition. Nature Chemical Biology, 6(8), 572-580.

Pastor, F., Kolonias, D., Giangrande, P. H., et al. (2010). Induction of tumour immunity by targeted inhibition of nonsense-mediated mRNA decay. Nature, 465(7295), 227-230.

Porter, D. L., Levine, B. L., Kalos, M., et al. (2011). Chimeric antigen receptor-modified T cells in chronic lymphoid leukemia. New England Journal of Medicine, 365(8), 725-733.

Reed, J. C. (2003). Apoptosis-targeted therapies for cancer. Cancer Cell, 3(1), 17-22.

Reits, E. A., Hodge, J. W., Herberts, C. A., et al. (2006). Radiation modulates the peptide repertoire, enhances MHC class I expression, and induces successful antitumor immunotherapy. The Journal of Experimental Medicine, 203(5), 1259-1271. 
Rezvani, K., \& Barrett, J. (2014). STAT3: the "Achilles" heel for AML? Blood, 123(1), 1-2.

Robins, H. I., Buchon, J. A., Varanasi, V. R., et al (1981). The abscopal effect: demonstration in lymphomatous involvement of kidneys. Medical and Pediatric Oncology, 9(5), 473-476.

Rosenblatt J, Stroopinsky D, Luptakova K, et al. (2012). Muc1 inhibition reverses the poor immunogenicity of leukemia stem cells rendering them susceptible to immunotherapy. Biology of Blood and Marrow Transplantation, 18(2), S319.

Schmiedel, B. J., Grosse-Hovest, L., \& Salih, H. R. (2013). A "vicious cycle" of NK-cell immune evasion in acute myeloid leukemia mediated by RANKL? Oncolmmunology, 2(5), e23850.

Seliger, B., Maeurer, M. J., \& Ferrone, S. (1997). TAP off-tumors on. Immunology Today, 18(6), 292-299.

Sioud, M. (2011). New insights into mesenchymal stromal cell-mediated T-cell suppression through galectins. Scandinavian Journal of Immunology, 73(2), 79-84.

Smits, E. L., Cools, N., Lion, E., et al. (2010). The toll-like receptor $7 / 8$ agonist resiquimod greatly increases the immunostimulatory capacity of human acute myeloid leukemia cells. Cancer Immunology, Immunotherapy, 59(1), 35-46.

Smits, E. L. J. M., Ponsaerts, P., Van de Velde, A. L. R., et al. (2007). Proinflammatory response of human leukemic cells to dsRNA transfection linked to activation of dendritic cells. Leukemia, 21(8), 1691-1699.

Somersan, S., Larsson, M., Fonteneau, J. F., et al. (2001). Primary tumor tissue lysates are enriched in heat shock proteins and induce the maturation of human dendritic cells. The Journal of Immunology, 167(9), 4844-4852.

Spranger, S., \& Gajewski, T. (2013). Rational combinations of immunotherapeutics that target discrete pathways. Journal for ImmunoTherapy of Cancer, 1, 1-14.

Stripecke, R., Levine, A. M., Pullarkat, V., et al. (2002). Immunotherapy with acute leukemia cells modified into antigen-presenting cells: ex vivo culture and gene transfer methods. Leukemia, 16(10), 1974-1983.

Tesniere, A., Panaretakis, T., Kepp, O., et al. (2008). Molecular characteristics of immunogenic cancer cell death. Cell Death \& Differentiation, 15(1), 3-12.

Uotila, P. (1996). The role of cyclic AMP and oxygen intermediates in the inhibition of cellular immunity in cancer. Cancer Immunology, Immunotherapy, 43(1), 1-9. 
Uyttenhove, C., Pilotte, L., Théate, I., et al. (2003). Evidence for a tumoral immune resistance mechanism based on tryptophan degradation by indoleamine 2, 3dioxygenase. Nature Medicine, 9(10), 1269-1274.

van Luijn, M. M., Chamuleau, M. E., Thompson, J. A., et al. (2010). Class II-associated invariant chain peptide down-modulation enhances the immunogenicity of myeloid leukemic blasts resulting in increased CD4+ T-cell responses. Haematologica, 95(3), 485-493.

van Luijn, M. M., van den Ancker, W., Chamuleau, M. E., et al. (2011). Absence of Class II-Associated Invariant Chain Peptide on Leukemic Blasts of Patients Promotes Activation of Autologous Leukemia-Reactive CD4+ T Cells. Cancer Research, 71(7), 2507-2517.

van Luijn, M. M., Chamuleau, M. E., Ressing, M. E., et al. (2010). Alternative liindependent antigen-processing pathway in leukemic blasts involves TAP-dependent peptide loading of HLA class II complexes. Cancer Immunology, Immunotherapy: CII, 59(12), 1825-1838.

van Luijn, M. M., van den Ancker, W., Chamuleau, M. E., et al. (2010). Impaired antigen presentation in neoplasia: basic mechanisms and implications for acute myeloid leukemia. Immunotherapy, 2(1), 85-97.

Vago, L., Perna, S. K., Zanussi, M., et al. (2009). Loss of mismatched HLA in leukemia after stem-cell transplantation. New England Journal of Medicine, 361(5), 478-488.

Verheyden, S., \& Demanet, C. (2008). NK cell receptors and their ligands in leukemia. Leukemia, 22(2), 249-257.

Vesely, M. D., Kershaw, M. H., Schreiber, R. D., et al. (2011). Natural innate and adaptive immunity to cancer. Annual Review of Immunology, 29, 235-271.

Vollmer, M., Li, L., Schmitt, A., et al. (2003). Expression of human leucocyte antigens and co-stimulatory molecules on blasts of patients with acute myeloid leukaemia. British Journal of Haematology, 120(6), 1000-1008.

Wiener, Z., Kohalmi, B., Pocza, P., et al. (2006). TIM-3 is expressed in melanoma cells and is upregulated in TGF-beta stimulated mast cells. Journal of Investigative Dermatology, 127(4), 906-914.

Weiss-Steider, B., Soto-Cruz, I., Martinez-Campos, C. A., et al. (2011). Expression of MICA, MICB and NKG2D in human leukemic myelomonocytic and cervical cancer cells. Journal of Experimental and Clinical Cancer Research, 30(1), 1-8. 
Wersäll, P. J., Blomgren, H., Pisa, P., et al. (2006). Regression of non-irradiated metastases after extracranial stereotactic radiotherapy in metastatic renal cell carcinoma. Acta Oncologica, 45(4), 493-497.

Weyhenmeyer, B., Murphy, A. C., Prehn, J. H., et al. (2012). Targeting the anti-apoptotic Bcl-2 family members for the treatment of cancer. Experimental Oncology, 34(3), 192-9.

Whiteside, T. L. (2002). Tumor-induced death of immune cells: its mechanisms and consequences. Seminars in Cancer Biology, 12(1), 43-50.

Whiteside, T. L. (2006). Immune suppression in cancer: effects on immune cells, mechanisms and future therapeutic intervention. Seminars in Cancer Biology, 16(1), 315.

Willingham, S. B., Volkmer, J. P., Gentles, A. J., et al. (2012). The CD47-signal regulatory protein alpha (SIRPa) interaction is a therapeutic target for human solid tumors. Proceedings of the National Academy of Sciences, 109(17), 6662-6667.

Woiciechowsky, A., Regn, S., Kolb, H. J., et al. (2001). Leukemic dendritic cells generated in the presence of FLT3 ligand have the capacity to stimulate an autologous leukemiaspecific cytotoxic $T$ cell response from patients with acute myeloid leukemia. Leukemia, 15(2), 246-255.

Yao, Y., Zhou, J., Wang, L., et al. (2013). Increased PRAME-specific CTL killing of acute myeloid leukemia cells by either a novel histone deacetylase inhibitor chidamide alone or combined treatment with decitabine. PloS One, 8(8), e70522.

Zitvogel, L., Apetoh, L., Ghiringhelli, F., et al. (2008). Immunological aspects of cancer chemotherapy. Nature Reviews Immunology, 8(1), 59-73. 


\section{Chapter III}

Cancer-induced impairments of immune cells and stromal cells; therapeutic strategies for repair 
When the immune system mounts an attack against cancer, tumor cells "fight back" by subverting anti-tumor immune defenses (Zitvogel, 2006). While Chapter II reviewed cancer-cell-derived factors that mediate immune evasion and suppression, this chapter describes immune-cell defects induced by cancer-distorted microenvironments. Intrinsic immune-cell defects (i.e. anergy, exhaustion, impaired maturation) and extrinsic immune-suppressing factors (i.e. TGF- $\beta$, IDO, CAFs) are reviewed. Potential ways to repair dysfunctional immune cells and counteract immuno-suppressive factors in cancer microenvironments are described. Tim-3 is highlighted because it disrupts immune responses and synapses, and both TGF- $\beta$ and RepSox affect Tim-3 (Wiener, 2007; Jajosky, 2014). In vivo Tim-3 blockade and the in vitro manipulation of Tim-3 expression by malignant cells warrant consideration when designing anti-cancer immune strategies.

\section{Generation of anti-tumor immune responses}

The immune system eliminates malignant cells via a variety of defenses including innate and adaptive immune cells, antibodies, complement proteins, and cytokines. Innate defenses are first to respond, followed by more specialized attack by antigenspecific $T$ and $B$ cells of the adaptive immune system. The key players of the innate and adaptive immune system are outlined in Figure 3.1. Adaptive immune responses, involving antigen-specific T and B cells or antibodies, can be divided into the "activation phase" involving tumor-antigen cross-presentation within lymphoid organs (i.e. lymph nodes) and the "effector phase" involving tumor-cell lysis in the periphery (Dzivenu, 2003; Yao, 2013). Figure 3.2 provides an overview of important soluble factors and cell-to-cell interactions that mediate generation of innate and adaptive anti-tumor immunity. 


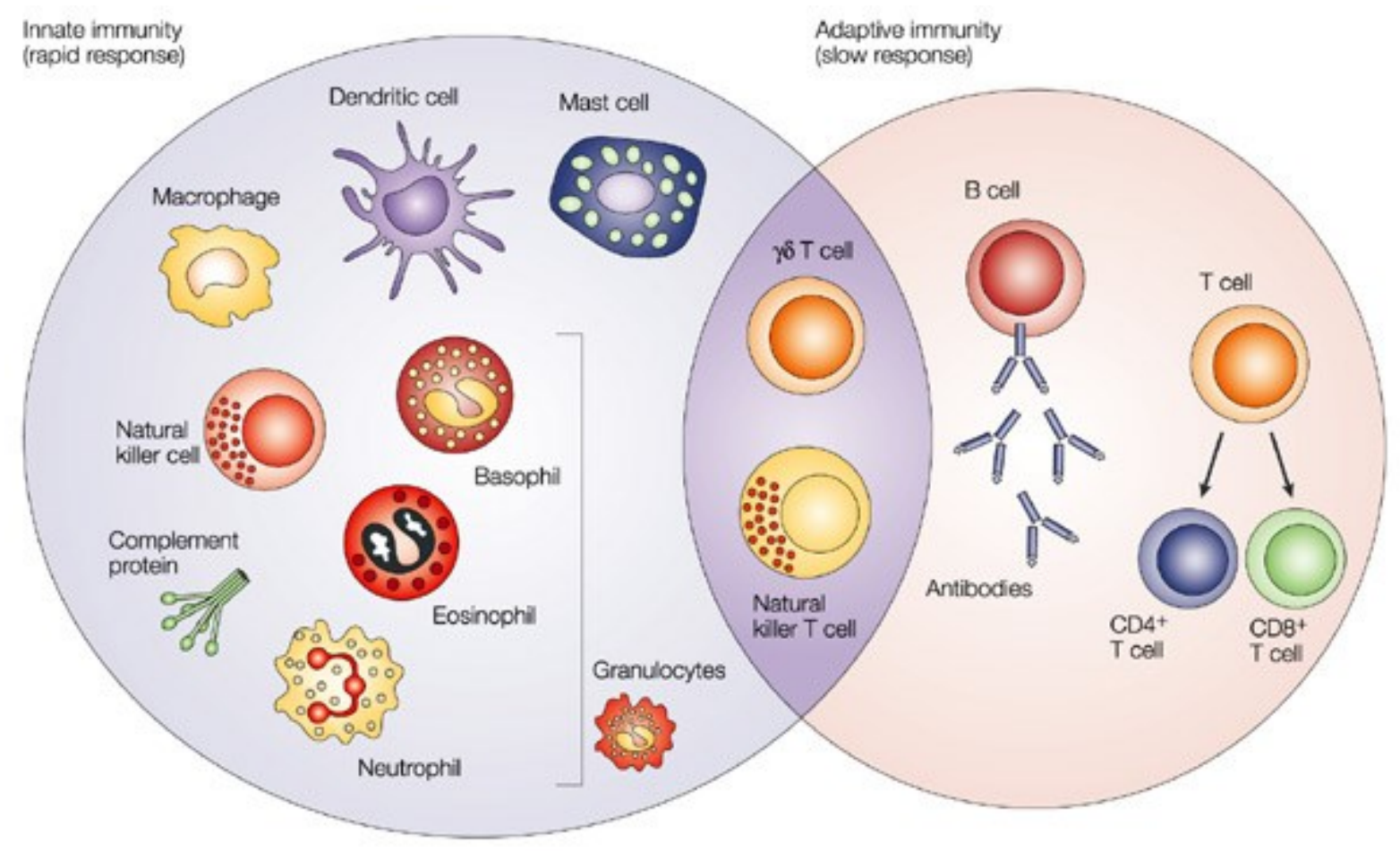

Figure 3.1. Innate and adaptive immune defenses. The innate immune system is the body's first line of defense against invading pathogens and cancer. Components of innate immunity include phagocytes (i.e. macrophages), NK cells, and complement proteins. The adaptive immune system generates antigen-specific anti-tumor responses mediated by antibodies, B cells, and T cells. Unlike innate immunity, the adaptive immune system can generate immunological memory that is capable of protecting against re-emergence of disease. Reproduced with permission from: Dranoff, G. (2004). Cytokines in cancer pathogenesis and cancer therapy. Nature Reviews Cancer, 4(1), 13. 

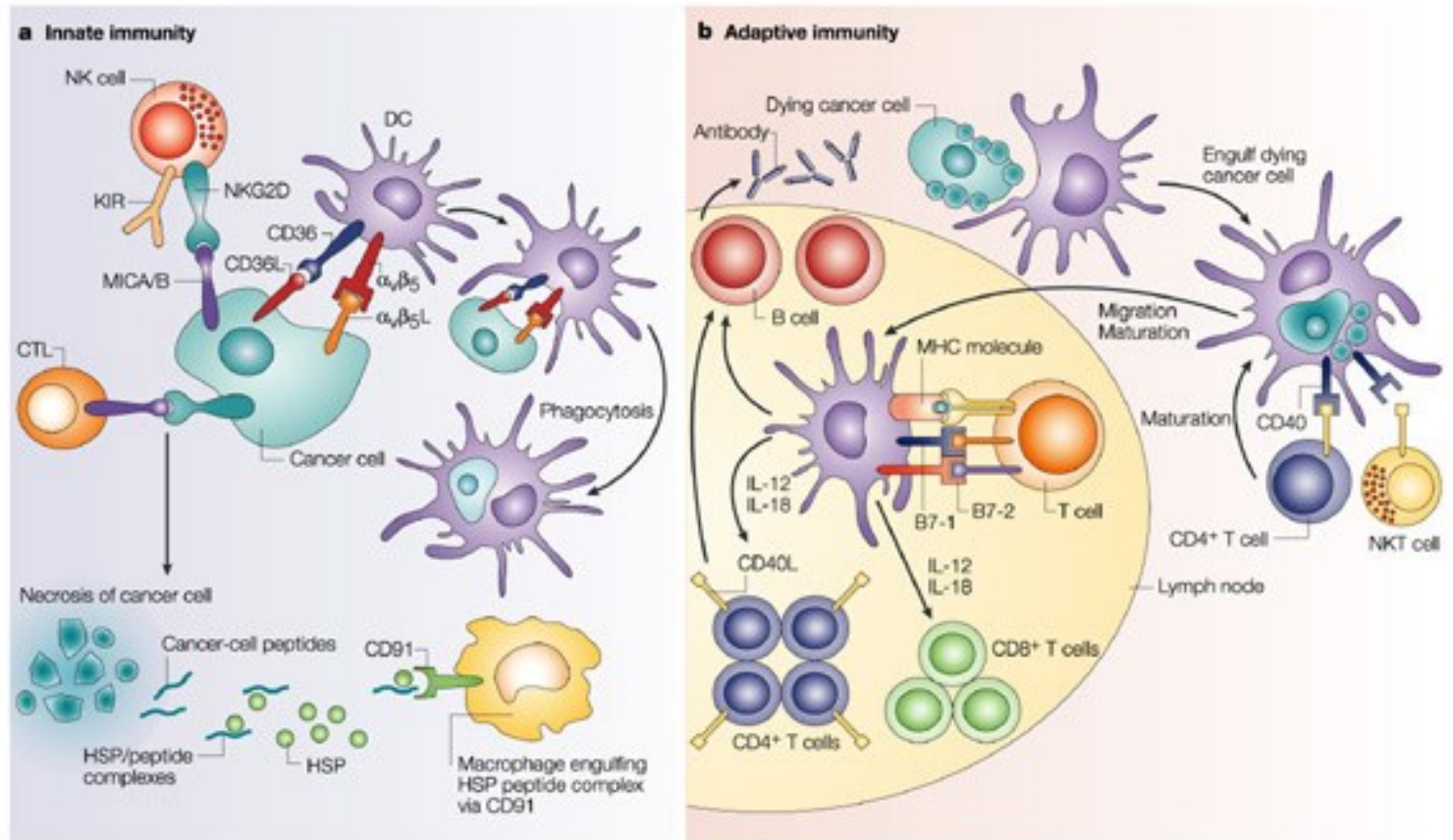

Figure 3.2. Generation of innate and adaptive anti-tumor immune responses. Within NK cells of the innate immune system, the net input of activating and inhibitory signals determines whether or not cancer cells lacking MHC class 1 molecules ("missing self") will be effectively killed. Within the adaptive immune system, DCs engulf tumor antigens and migrate to lymph nodes where they present tumor peptide-MHC complexes to T cells (a process called "cross-priming"). Activated CD4 ${ }^{+} \mathrm{T}_{\mathrm{H}}$ cells, in turn, help to activate CD8+ cytotoxic $T$ cells and stimulate B cells to produce antibodies. Reproduced with permission from: Dranoff, G. (2004). Cytokines in cancer pathogenesis and cancer therapy. Nature Reviews Cancer, 4(1), 14. 


\section{Activation phase}

Antigen-presenting cells (APCs), such as macrophages and DCs, engulf cancer cells or capture antigens released by dying cells. Immunogenic tumor-cell death, illustrated in Figure 2.4, activates APCs through release of pro-inflammatory "find me," "eat me," and "danger" signals (Tesniere, 2008; Inoue, 2014). In contrast, nonimmunogenic cell death promotes tolerance. APCs process and present captured tumor antigens on their surface via major histocompatibility complex (MHC) class II molecules

(Dzivenu, 2003). After migrating to tumor-draining lymph nodes, DCs cross-present malignant peptide-MHC complexes to $\mathrm{CD}^{+}{ }^{+} \mathrm{Th}$ cells (Dzivenu, 2003). Th cells secrete cytokines that activate other immune cells. Th cells can be divided into type 1 (Th1) and type $2\left(T_{h} 2\right)$ cells based on the cytokines they secrete. By secreting interferon-gamma (IFN- $\mathrm{Y}$ ) and IL-2, $\mathrm{Th}_{\mathrm{h}} 1$ cells activate $\mathrm{CD} 8^{+}$cytotoxic $\mathrm{T}$ cells and promote cell-mediated immunity (Dzivenu, 2003). In contrast, by secreting IL-4 and IL-5, $\mathrm{Th}_{\mathrm{h}}$ cells stimulate antibody production by B cells (Dzivenu, 2003).

\section{Effector phase}

Once activated, cytotoxic $T$ cells recognize malignant cells expressing cancerspecific or cancer-associated antigens bound to MHC class 1 molecules via their T-cell receptor (Dzivenu, 2003). Cytotoxic T cells lyse cancer cells by releasing perforins and granzymes. Once activated, B cells differentiate into antibody-producing plasma cells. Antibodies bind the surface of cancer cells and lead to tumor-cell death through antibodydependent cellular cytotoxicity (ADCC) or activation of the complement cascade (Dzivenu, 2003). ADCC involves recruitment of immune cells, such as macrophages and NK cells, which engulf or lyse cancer cells (Raval, 2014). Antibodies can also activate 
the complement cascade which, in turn, induces tumor-cell lysis by perforating the cell membrane (Raval, 2014).

\section{Cancer immunoediting hypothesis}

The cancer immunoediting hypothesis (see Figures 3.3 and 3.4 ) describes how the immune system affects cancer development through its dual host-protective and tumor-promoting roles (Dunn, 2002; Dunn, 2004). Immunoediting of tumors can be divided into three phases: elimination, equilibrium, and escape - see Figure 3.4 (Dunn, 2002; Dunn, 2004). During the elimination (“immuno-surveillance") phase, immunogenic tumor cells are eliminated by innate and adaptive immune cells. If all cancer cells are eradicated, disease is cured. If not, residual "immune-escape variants" remain behind. During the equilibrium ("immuno-selection") phase, tumor outgrowth is prevented, but remaining cancer cells continue to evolve under constant immune-selection pressures. During the escape ("immuno-subversion") phase, the poorly immunogenic cancer cells that evaded immune destruction grow and expand. 


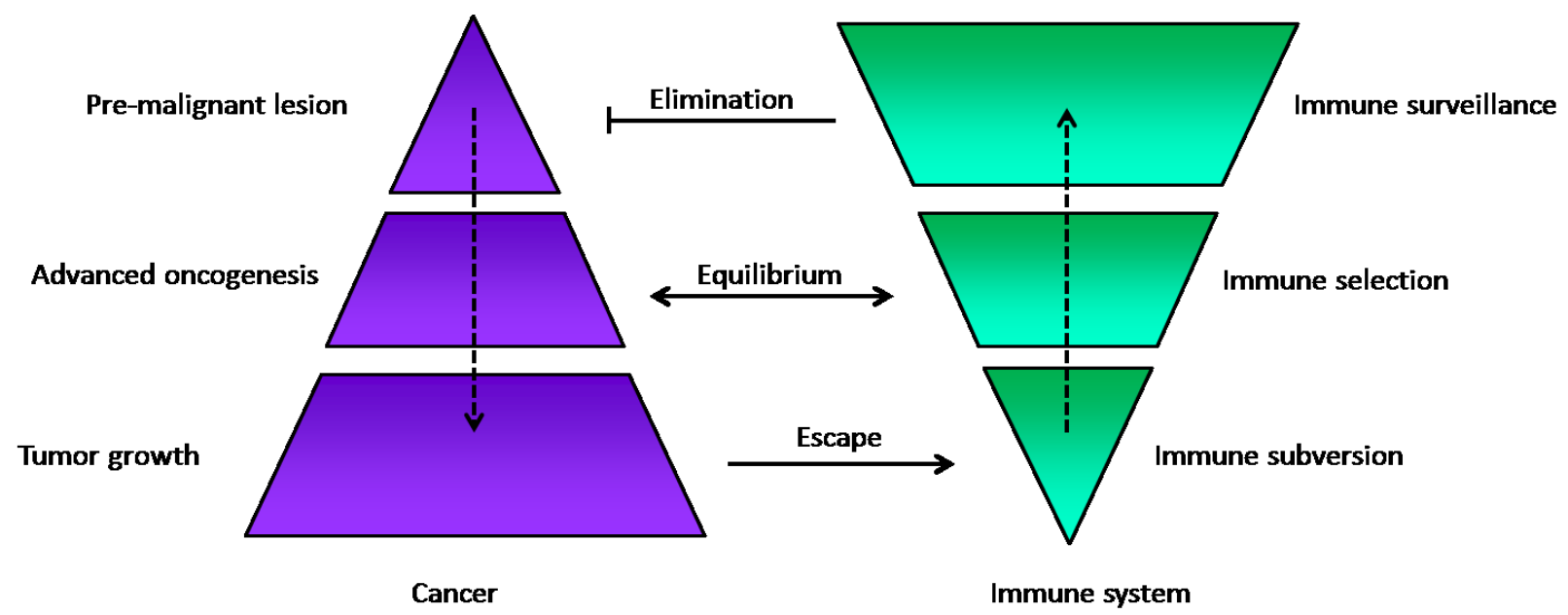

Figure 3.3. Immunoselection and immunosubversion during tumor evolution. By eliminating immunogenic tumor cells, the immune system selects for poorly immunogenic tumor cells that escape immune recognition. These immune escape variants progressively alter ("hijack") their microenvironment to suppress immune defenses and promote tumor growth. Adapted with permission from: Zitvogel, L., Tesniere, A., \& Kroemer, G. (2006). Cancer despite immunosurveillance: immunoselection and immunosubversion. Nature Reviews Immunology, 6(10), 716. 


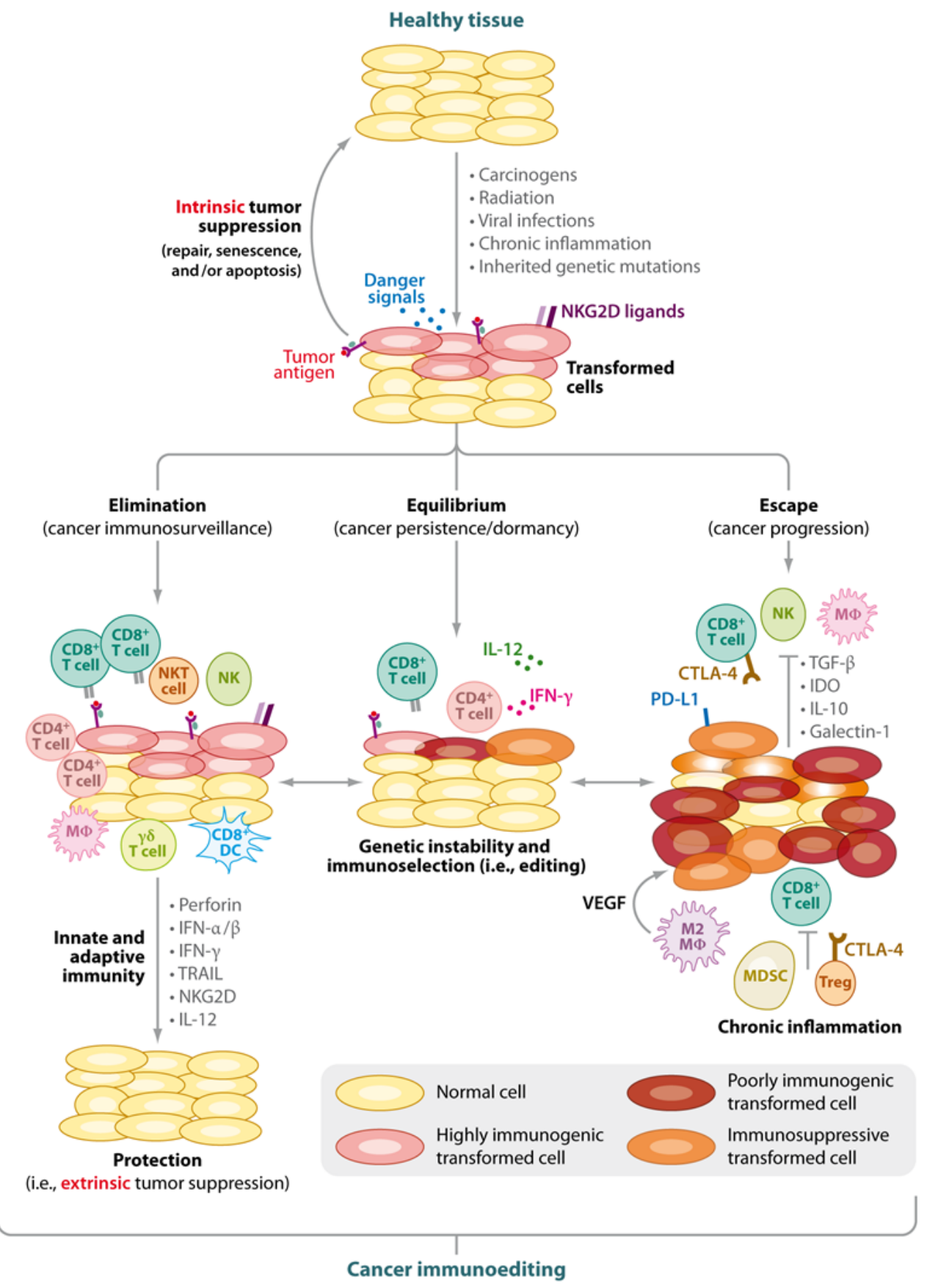

Figure 3.4. Three phases of cancer immunoediting: elimination, equilibrium, and escape. As cancers evolve, tumor cells are recognized and cleared by the immune system. Elimination of all tumor cells cures disease. If malignant disease persists, the immune system may prevent outgrowth of surviving tumor cells for some time. However, tumor cells may continue to evolve and alter surrounding tissues in ways that impair immune defenses. Ultimately, tumor cell "immune escape" variants may emerge and cause overt disease. Reproduced with permission from: Vesely, M. D., Kershaw, M. H., Schreiber, R. D., et al. (2011). Natural innate and adaptive immunity to cancer. Annual Review of Immunology, 29, 238. 


\section{Cancer-induced dysregulation of the immune system}

As cancers progress, immune cells may no longer be recruited to, or activated by, malignant cells; and immune-cell function may be inhibited by tumor microenvironments (Gajewski, 2006; Spranger, 2013). Even when patients do not generate an effective anticancer immune response, tumor-reactive immune cells may still exist, but are, unfortunately, overwhelmed or suppressed (Gajewski, 2006; Tran, 2014). Immunotherapies for cancer aim to trigger de novo immune responses and enhance ("rescue") pre-existing anti-tumor immune reactions that have become silenced (Mellman,

2011). Conceptually, this involves stimulating the immune-activation phase and rescuing the immune-effector phase. Remarkably, cancer-induced immune-cell defects can be repaired (Hodi, 2010; Reiners, 2013; Vizcardo, 2013), and immuno-suppressive cells, enzymes, and soluble factors can be neutralized or eliminated (van den Boorn, 2013). Thus, it is possible to convert non-immunogenic tumor microenvironments into anticancer, immuno-stimulating tissues (van den Boorn, 2013).

\section{Cancer-induced intrinsic immune-cell defects}

Unfortunately, dysfunctional immune cells can accumulate in cancer patients (Gabrilovich, 2004; Pinzon-Charry, 2005; Wherry, 2011). Defects include anergy, exhaustion, impaired maturation, deletion, and unfavorable polarization (toward type 2 humoral immunity rather than type 1 cell-mediated immunity). Immune-effector cells become "tolerant" and are unable to eliminate cancer cells (Crespo, 2013; Wherry, 2011). By failing to adequately present antigens and activate T cells, the (immature) DCs actually promote T-cell tolerance (Lutz, 2002; Dudek, 2013). Cancer-induced dysfunctions of T cells, DCs, NK cells and macrophages are reviewed. 


\section{T-cell defects}

In patients with cancer and chronic viral infections, $T$ cells can become dysfunctional (i.e. anergic, exhausted) or may be deleted. T-cell anergy is due to incomplete activation (i.e. T-cell receptor ligation in the absence of costimulatory signals like CD28 engagement) (Chappert, 2010), while exhaustion arises from chronic overstimulation (Wherry, 2011). Exhausted T cells often express inhibitory immunecheckpoint receptors (Tim-3 and PD-1) and display impaired cytokine secretion and cytolytic activity upon antigen stimulation (Wherry, 2011). Compared to healthy controls, T cells from AML patients formed defective immune synapses with AML cells and had (altered) gene expressions consistent with impaired cytolytic activity (Le Dieu, 2009).

\section{NK-cell defects}

Cancer-cell-induced NK-cell abnormalities include impaired maturation, anergy, decreased cytotoxicity, and defective secretion of cytokines like IFN-ץ (Das, 2000; Richards, 2006; Lion, 2012). Compared to patients without cancer, NK cells from AML patients have decreased expression of activating NK-cell receptors and increased expression of inhibitory NK-cell receptors (Costello, 2002; Lion, 2012). This unfavorable balance of stimulatory and inhibitory receptors may prevent effective NK-cell-mediated elimination of AML cells.

\section{$D C$ defects}

In cancer patients, incomplete maturation of DCs is one reason why immune responses are impaired (Pinzon-Charry, 2005). Tumor-derived factors, such as gangliosides, VEGF, IL-6, and IL-10, inhibit DC differentiation (Gabrilovich, 2004). This leads to an accumulation of immature DCs and the absence of an adequate number of 
functional (mature) DCs as well as an accumulation of immature myeloid progenitors generally - a combination of adverse effects which, collectively, can substantially impair T-cell responses (Gabrilovich, 2004). Of note, immature (dysfunctional) DCs are among the myeloid-derived suppressor cells (MDSCs) that suppress T-cell immunity - see Figure 3.5 (Gabrilovich, 2009; Khaled, 2013; Ostrand-Rosenberg, 2009). That is, MDSCs include a variety of immature myeloid cells that have not fully differentiated into fully functional macrophages, DCs, or granulocytes (Gabrilovich, 2009; Khaled, 2013). Because immature DCs present antigen in the absence of appropriate costimulatory signals (i.e. CD80 and CD86) and/or proinflammatory cytokines, they, unfortunately, induce T-cell tolerance rather than protective anti-tumor responses (Gabrilovich, 2004).

\section{Macrophage defects}

Tumor-associated macrophages (TAMs) accumulate in tumors, and high tumor influx of TAMs is associated with poor prognosis in cancer patients (Solinas, 2009; Tang, 2013). TAMs may polarize to type 2 (M2) macrophages that promote tumor growth (Solinas, 2009). While type 1 (M1) macrophages produce IL-12 and enhance $T_{h} 1$ and NK cell-mediated immunity, M2 macrophages secrete IL-10 and promote $T_{h} 2$ humoral immunity - see Figure 3.5 (Solinas, 2009). Of note, cell-mediated immunity is believed to be more effective than antibodies in controlling tumor growth.

\section{Strategies to repair dysfunctional immune cells}

Remarkably, dysfunctional immune cells can be repaired ("re-educated" or "reprogrammed") and then activated and expanded to promote tumor-rejection. Using a metastatic melanoma patient's exhausted $\mathrm{CD} 8^{+} \mathrm{T}$ cells, reprogramming to pluripotency followed by re-differentiation - generated "rejuvenated" cytotoxic $T$ cells that were 
functional based on their ability to produce IFN-y upon encounter with antigen (Vizcardo, 2013). Immature DCs can be differentiated into functional APCs using ATRA (Mirza, 2006; Kusmartsev, 2003). In a mouse model of breast cancer, type 2 tumor-promoting TAMs were re-polarized into type 1 cells with increased tumoricidal activity using a combination of microbial stimulus (TLR9 ligand CpG) and anti-IL-10-receptor antibody (Giuducci, 2005). Here, strategies are reviewed that can repair and activate defective immune-effector cells in order to stimulate anti-tumor immunity. Relevant factors include proinflammatory cytokines, "danger signals" (like TLR agonists), and antibodies that activate costimulatory, and/or block coinhibitory, immune-checkpoint receptors.

\section{Repairing T cells}

Several strategies have been developed to reverse T-cell tolerance. Examples include targeting immune-checkpoint receptors, delivering proinflammatory cytokines, and reprogramming T cells to pluripotency (Pardoll, 2012; Vizcardo, 2013).

\section{Modulation of immune-checkpoint receptors}

Antibodies that activate costimulatory receptors or inhibit immune-checkpoint receptors can stimulate $T$ cells. For example, inhibiting the PD-1 signaling pathway can rescue exhausted T cells in the setting of cancer and chronic viral infection (Barber, 2006; Zitvogel, 2012). Co-expression of PD-1 and Tim-3 identifies "exhausted" killer T cells in mice with AML (Zhou, 2011), and co-blockade of PD-1 and Tim-3 more effectively stimulates anti-tumor immunity than either therapy alone (Sakuishi, 2010). In clinical trials, PD-1 blockade induced tumor regression in patients with advanced melanoma or lung and kidney cancers (Brahmer, 2012; Topalian, 2012). Treatment with ipilimumab, a CTLA-4 blocking antibody, restored T-cell anti-tumor immunity and improved survival of 
melanoma patients (Hodi, 2010). Antibody activation of the costimulatory receptor OX40 (Weinberg, 2011) and blockade of the coinhibitory receptor LAG-3 (Goldberg, 2011) can also promote anti-tumor immunity.

\section{Cytokine stimulation}

Exposure to cytokines, including IL-15, IL-17 and IL-2, can prevent, or reverse, T-cell anergy induced by tumors and chronic viral infections (Boussiotis 1994; Gu, 2007; Teague, 2006). Using a mouse model of T-cell tolerance, in vitro exposure to IL-15 rescued "tolerant" $\mathrm{T}$ cells; furthermore, adoptive transfer of rejuvenated $\mathrm{T}$ cells was effective in treating mice with leukemia (Teague, 2006).

\section{Induction of homeostatic proliferation}

Lymphopenia-induced homeostatic T-cell proliferation can reverse tumor-induced T-cell anergy and promote tumor rejection (Kline, 2008; Brown, 2006). Lymphopenic conditioning of patients prior to receiving adoptively transferred $T$ cells improves the persistence and expansion of infused T cells (Dudley, 2002; Rosenberg, 2008). Presumably, lymphopenic conditioning depletes $T_{\text {regs }}$ and supports engraftment and homeostatic proliferation of therapeutic (functional) T cells.

\section{Repairing NK cells}

Following exposure to IL-15, NK cells from AML patients show increased expression of activating receptors and improved cytotoxicity (Szczepanski, 2010). IL-15 may also promote NK-cell maturation (Richards, 2006). Anti-KIR antibodies block inhibitory NK-cell receptors and promote NK-cell-mediated killing of tumor cells (Romagne, 2009; Velardi, 2009; Alici, 2010). NK-cell-mediated cytotoxicity against non- 
Hodgkin's lymphoma tumor cells improved with the use of bispecific antibodies that could crosslink the CD16A receptor on NK cells with the CD30 receptor on non-Hodgkin's lymphoma cells (Reiners, 2013). Targeted anti-cancer therapies can also promote the anti-tumor activity of NK cells. For example, imatinib increases DC-mediated NK-cell activation (Borg, 2004).

\section{Role of Tim-3 expression in immune-cell dysfunction}

Tim-3 inhibits the pro-inflammatory or anti-tumor activities of a variety of immune cells, including $\mathrm{T}, \mathrm{NK}$, dendritic, and monocytic cells. The endothelial and stromal cells that surround tumors also mediate immune suppression. Tim-3-expressing endothelial cells may function as an immunological barrier shielding tumor cells from infiltrating T cells by directly suppressing their activity (Huang, 2010). Tumor cells may convert stromal cells into galectin-9-expressing CAFs capable of suppressing T-cell responses (Gieseke, 2013; Sioud, 2013). Similar to the adverse consequences of Tim-3 expression by immune and stromal cells, Tim-3 expression by tumor cells can also promote immune escape or suppression by, for example, disrupting cancer-cell/immune-cell synapses.

\section{Cytotoxic $T$ cells}

Tim-3 promotes T-cell tolerance in the setting of cancer and chronic viral infections (Sánchez-Fueyo, 2003). High Tim- $3^{+} \mathrm{T}$ cells do not respond to stimulation: they are unable to proliferate or produce cytokines. Thus, high Tim- $3^{+} \mathrm{T}$ cells are known as "noneffector" or "exhausted" T cells (Golden-Mason, 2009; Hastings, 2009). In lymphoma patients, T-cell dysfunction ("exhaustion") was found to be mediated, in part, by increased Tim-3 expression by T cells (Yang, 2012). In the context of chronic viral infections, Tim- $3^{+}$ T cells displayed impaired secretion of the cytokines IFN- $y$ and TNF- $\alpha$ and were more 
prone to apoptosis (Jones, 2008; Golden-Mason, 2009; Jin, 2010; Ju, 2009). Tim-3 expression on the surface of $\mathrm{CD}^{+} \mathrm{T}$ cells impairs their cytotoxicity by inhibiting the degranulation needed for the secretion of perforin and granzymes (Sakhdari, 2012). Cytokine production by $T_{h} 1$ cells in mice is enhanced following treatment with Tim-3 blocking antibodies (Sabatos, 2003). In the presence of Tim-3 blocking antibodies, CD4 ${ }^{+}$ T cells showed enhanced secretion of the proinflammatory cytokines IFN- $\gamma$, IL-2, IL-17, and IL-6 (Hastings, 2009). In vitro, the Tim-3 ligand galectin-9 blocks differentiation of naïve $T_{\text {cells into }} \mathrm{Th}_{\mathrm{h}} 17$ cells while inducing their differentiation into suppressive $\mathrm{T}_{\text {regs }}$ (Seki, 2008). Unfortunately, over-expression of Tim-3 can emerge as malignant disease progresses, and Tim-3 can promote immune tolerance by (adversely) altering the malignant, immune, and stromal cells of cancer patients. Tim-3 blockade can restore anti-tumor immunity by inducing therapeutically beneficial changes in multiple cell types.

Regulatory $T$ cells ( $\left.T_{\text {regs }}\right)$

Suppressive $T_{\text {regs }}$ inhibit anti-tumor immunity, and in a mouse model of colon

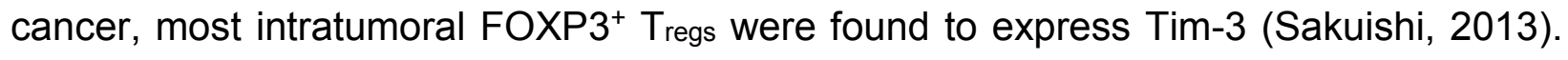
Tim- $3^{+} T_{\text {regs }}$ that co-express PD-1 are highly suppressive (Sakuishi, 2013). As an anticancer strategy, the benefit of simultaneously blocking both the Tim-3 and PD-1 signal transduction cascades likely arises not only from the reversal of T-cell exhaustion, but also from the inhibition of Treg-cell function (Sakuishi, 2013). It seems Tim-3 identifies those $T_{\text {regs }}$ that very strongly suppress anti-tumor immune responses (Sakuishi, 2013).

\section{Monocytes and Macrophages}

Tim-3 expression by monocytes and macrophages has not been well studied in cancer. Blocking Tim-3 receptors on monocytes increased their secretion of IL-12, a 
cytokine that promotes $\mathrm{Th}_{\mathrm{h}} 1$ and NK-cell-mediated immunity (Zhang, 2012). In mice with sepsis, Tim-3 blockade increased macrophage activation (accompanied by increased mRNA expression of proinflammatory cytokines and TLRs) and exacerbated sepsis, suggesting Tim-3 can limit macrophage activity (Yang, 2013). Of note, activation of the Tim-3-galectin 9 pathway has also been shown to promote macrophage bactericidal activity (Sada-Ovalle, 2012).

\section{Dendritic cells}

In mice with lung or colon cancer, Tim-3 was over-expressed on tumor-infiltrating DCs compared to the DCs residing within normal tissues and the DCs of healthy mice (Chiba, 2012). These high Tim-3-expressing DCs enable tumors to evade immuno-

surveillance by suppressing the sensing of nucleic acids associated with by tumorinduced inflammation (Chiba, 2012; Jinushi, 2012).

Natural killer cells (NK cells)

Although Tim $-3^{+}$NK cells from healthy donors were mature and fully functional, Tim-3 may suppress NK-cell-mediated cytotoxicity upon encounter with target cells expressing Tim-3 ligands (Ndhlovu, 2012). Relative to NK cells from healthy donors, NK cells from melanoma patients displayed elevated Tim-3 expression along with reduced cytotoxicity, IFN-ץ secretion, and proliferation (da Silva, 2014). Among melanoma patients, Tim-3 expression on NK cells was generally higher during advanced stages of disease and in patients with poor prognostic factors (da Silva, 2014). Furthermore, Tim-3 blockade reversed NK-cell exhaustion and improved cytotoxicity against melanoma cell lines (da Silva, 2014). 
Natural killer-T (NKT) cells

Tim- $3^{+}$NKT cells in the liver are functional: they can proliferate and produce cytokines when stimulated. When liver NKT cells are activated by exogenous bacterial antigens, Tim-3 expression is upregulated and IFN- $\gamma$ is secreted. IFN- $\gamma$ induces production of galectin-9 by Kupffer cells in the liver which, in turn, induces apoptosis of Tim $-3^{+}$NKT cells and reduces the inflammatory response. That is, in some molecular and cellular contexts, Tim-3 expression on NKT cells leads to NKT-cell apoptosis which, in turn, limits the NKT-cell immune response (Kinjo, 2005; Mattner, 2005; Kinjo, 2006; Mengshol, 2010).

Tim-3 blockade to stimulate immune-cell activity

Because Tim-3 restrains anti-tumor immunity, preclinical studies are evaluating the use of Tim-3 blockade to promote immune-mediated tumor-rejection. Along with PD-1 blockade (Sakuishi, 2010) and CD137 activation (Guo, 2013), Tim-3 blockade reverses T-cell exhaustion and promotes anti-tumor immunity in mice. Co-blockade of Tim-3 and Tim-4 also improved vaccine-induced anti-tumor immune responses by stimulating NK and T-cell activity in mice with melanoma (Baghdadi, 2013). Based on these successful animal trials, Tim-3 blocking antibodies are in preclinical development.

\section{Cancer-induced extrinsic immune suppression}

Tumors recruit, and induce the formation of, suppressive immune cells. Because inhibitory (aka "regulatory") immune cells normally dampen immune responses, they may be recruited and "hijacked" by tumors to promote immune escape (Zitvogel, 2006). Under normal physiologic conditions, inhibitory cells (like $T_{\text {regs }}$ ) are useful because they limit chronic inflammation, maintain peripheral tolerance to self antigens, and prevent 
autoimmune disease (Hori, 2003; Vignali, 2008). But, in the context of malignant disease, Figure 3.5 shows how Tregs, tolerogenic DCs, tumor-associated (M2) macrophages, MDSCs, and CAFs facilitate tumor progression by suppressing the immune system. Regarding the generation of inhibitory immune cells, tumors impair the maturation of myeloid cells into functional anti-tumor effector cells and skew development of immune responses toward those that are antibody-based and away from the more effective, cellmediated responses (Gabrilovich, 2004; Pinzon-Charry, 2005; Solinas, 2009). Tumors also secrete TGF- $\beta$ which can convert T cells into $T_{\text {regs }}$ by inducing FoxP3 expression (Liu, 2007). Regarding the adverse role played by support cells in tumor-cell microenvironments, cancers distort local stromal and endothelial cells in ways that suppress the infiltrating immune cells (i.e. by inducing expression of Tim-3 or galectins) (Huang, 2010; Mellman, 2011; Gieseke, 2013; Sioud, 2013). 


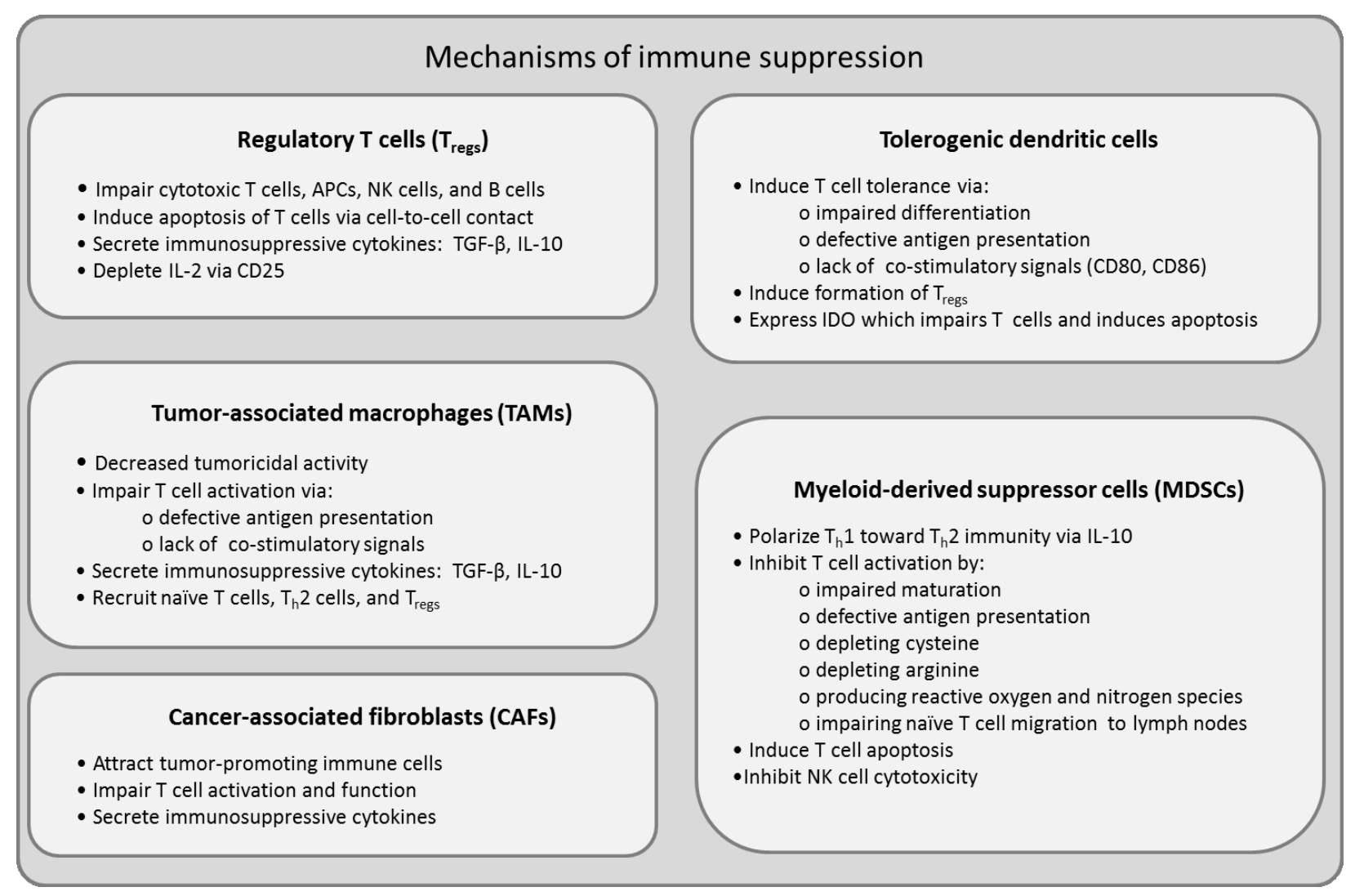

Figure 3.5. Proposed mechanisms of immune suppression by immune cells and cancer-associated fibroblasts. Adapted with permission from: (1) Kerkar, S. P., \& Restifo, N. P. (2012). Cellular constituents of immune escape within the tumor microenvironment. Cancer Research, 72(13), 3126. and (2) Baitsch, L., Fuertes-Marraco, S. A., Legat, A., et al. (2012). The three main stumbling blocks for anti-cancer T cells. Trends in Immunology, 33(7), 365. 


\section{Reversing immune suppression}

Strategies to reverse immuno-suppression include eliminating suppressive cells or their secretions, inducing immature ("tolerogenic") cells to differentiate into functional cells, and repolarizing ("re-educating") immune responses from tumor-promoting to tumor-rejecting responses. Inhibitory immune cells can be depleted using targeted therapies or systemic agents with immunological effects (Zou, 2006; Vanneman, 2012).

\section{Targeting $T_{\text {regs }}$}

Therapeutic strategies for targeting $T_{\text {regs }}$ have been previously reviewed (Zou, 2006). Daclizumab, a CD25-targeting monoclonal antibody, depletes $T_{\text {regs }}$ (which are classically $\mathrm{CD} 4^{+} \mathrm{CD} 25^{+} \mathrm{FoxP}^{+}$) and improves anti-tumor immunity in preclinical studies (Kline, 2008) and in clinical trials (Rech, 2009; Rech 2012). Denileukin difitox (OnTak), an IL-2-diphtheria toxin fusion protein, reduces $T_{\text {regs }}$ and induces tumor regression in patients with melanoma (Telang, 2011). Glucocorticoid-induced tumor-necrosis factorreceptor related protein (GITR)-stimulating antibodies reduced the tumor-suppressor activity of Tregs in vitro (Shimizu, 2002) and promoted tumor regression in mice (Ko, 2005).

\section{Targeting tolerogenic DCs}

Therapeutic targeting of immature tolerogenic DCs can involve eliminating them, inhibiting their formation or expansion, or promoting their differentiation into functional DCs (that can present tumor antigens, secrete proinflammatory cytokines, express costimulatory ligands, and activate $T$ cells). In mice with AML, treatment with agonistic CD40 antibodies - which are known to activate host APCs like DCs - reversed T-cell tolerance and prolonged survival (Zhang, 2013). CD40 ligation also restored T-cell antimicrobial immunity (Tuma, 2002). All-trans-retinoic-acid (ATRA) differentiated 
immature myeloid cells into mature DCs (as well as macrophages and granulocytes) and improved anti-tumor immunity in tumor-bearing mice upon vaccination (Kusmartsev, 2003). Because immature DCs are a type of MDSC, see the "Targeting MDSCs" section for other ways to therapeutically target these cells.

\section{Targeting tumor-associated macrophages (TAMs)}

Therapeutic targeting of TAMs (including strategies to inhibit recruitment to tumors, impair survival, increase M1-like tumor-killing activity, and reduce M2-like tumorpromoting activity) have been extensively reviewed (Tang, 2012). Therapies may seek to reduce the number of TAMs or shift ("repolarize") macrophage behavior from tumorpromoting to tumor-rejecting agendas (Biswas, 2010). Combining the TLR9 agonist CpG with IL-10-receptor-blocking antibodies reprogrammed M1-like TAMs to potent M2-like anti-tumor effectors (Guiducci, 2005). CD40 agonistic antibodies (Buhtoiarov, 2006; Buhtoiarov, 2011; Beatty, 2011) and IL-12 (Watkins, 2007) also enhanced the anti-tumor activity of M1-like TAMs. Exposure to histidine-rich glycoprotein (Rolny, 2011) and inhibition of STAT3 and STAT6 signaling (Kortylewski, 2005; Cheng, 2003) reduced M2like TAM tumor-promoting activity and inhibited tumor growth.

\section{Targeting myeloid-derived suppressor cells (MDSCs)}

Strategies that target MDSCs - by inducing differentiation, preventing expansion, inhibiting function, or eliminating them - have been extensively reviewed (Gabrilovich, 2009). Typically, the goal is to reduce the number of MDSCs or alter their function. ATRA (Mirza, 2006; Kusmartsev, 2003) and 25-hydroxyvitamin D3 (Lathers, 2004) promote MDSC differentiation into mature macrophages or DCs. Nitroaspirin (De Santo, 2005), sildenafil (Serafini, 2006), and biphosphonate (Melani, 2007) inhibit MDSC activity. 
Inhibiting stem-cell-factor-mediated signaling reduces MDSC expansion (Pan, 2008). Some chemotherapy drugs like gemcitabine eliminate MDSCs (Suzuki, 2005).

Targeting suppressive metabolic enzymes

Anti-cancer therapies are being developed to not only eliminate inhibitory immune cells, but also to reduce their immuno-suppressive secretions. Enzymes like IDO and arginase impair the anti-tumor activity of NK and T cells by depleting tryptophan and arginine - amino acids needed for T-cell proliferation and function. Inhibiting IDO and arginase activity improves anti-tumor immunity in preclinical models (Uyttenhove, 2003; Liu, 2010; Raber, 2012) and is now being investigated in clinical trials. In normal physiologic settings, IDO limits the activation of T cells and NK cells in the placenta (Frumento, 2002; Munn, 2002).

\section{General non-targeted strategies}

The physical removal of malignant cells has been shown to reduce immunosuppression. Removal of primary tumors restored humoral and cell-mediated anti-tumor immunity in mice with breast cancer despite persistence of extensive metastatic disease (Danna, 2004). Presumably, surgery not only eliminates cancer cells and the immunosuppressive factors they secrete, but also a variety of inhibitory immune cells that have infiltrated the tumor. Removing both tumor cells and tumor-promoting immune cells may tip the balance toward effective anti-tumor immunity. Perhaps this means leukemia patients are most likely to respond to immunotherapy in the setting of MRD. Of note, lymphodepleting regimens (that reduce the number of $\mathrm{T}_{\text {regs }}$ ) promote expansion of adoptively transferred T cells by inducing their homeostatic proliferation (Dudley, 2002; Rosenberg, 2008). Thus, the numbers of cancer and immune-effector cells - or the 
immune-effector/immune-suppressor cell ratio - may determine whether malignant cells can persist or will be eliminated.

Overall, in addition to inducing immunogenic tumor-cell death, some standard ("conventional") anti-cancer therapies may have additional immuno-stimulatory effects (Zitvogel, 2008). For example, chemotherapeutic agents like cyclophosphamide can partially deplete or inactivate $T_{\text {regs }}$ (Ghiringhelli, 2004; Lutsiak, 2005).

\section{Cancer-associated fibroblasts}

The conceptual frameworks that are driving cancer research include theories and models that integrate diverse scientific findings and viewpoints. This is especially true for the study of cancer microenvironments. Experiments that are clarifying how local surroundings support tumor cells have been inspired by provocative models of tumormicroenvironment interactions (Infanger, 2013). For example, some propose that the physiologic mechanisms that so effectively protect cancer cells had originally evolved to promote wound-healing and regeneration (Oviedo, 2009). In this conceptual framework, some patterns of inflammation are critical. In response to injury, tissue repair in primitive animals occurs in adverse acidic, hypoxic, inflammatory microenvironments that trigger mechanisms that promote the survival and proliferation of critical stem-like (normal) cells that regenerate tissues. Cancer-induced inflammatory states resemble those triggered by injury, and they may trigger the same cell-protection mechanisms that evolved to protect normal stem cells to, unfortunately, promote CSC survival (Landsberg, 2012). In this discussion, (from among many synonyms) the terms "normal stromal fibroblast" and "CAF" are used. As shown in Figure 3.6, tumor microenvironments convert, or reprogram, normal stromal fibroblasts into stromal cells with a CAF phenotype. These CAFs, in turn, 
alter the phenotypes of immune cells in ways that promote tumor-cell survival via immune evasion and tolerance. Of note, the terms "mesenchymal stem cells" and "multi-potent mesenchymal stromal cells" are other names that are sometimes used for the cells that give rise to normal stromal fibroblasts. For simplicity, this discussion uses an example involving "the reciprocal-interactions model for CAF creation," the galectin-9/Tim-3 axis, and the secretion of pro-inflammatory cytokine IL-6 to illustrate one way the interactions of cells and molecular factors may cause immune cells to become dysfunctional in cancer patients. The "reciprocal-interactions model" proposes that tumor cells attract macrophages and, together, these cells convert, or reprogram, normal stromal fibroblasts into CAFs via pro-inflammatory cytokines like IL-6 (Haviv, 2009; Anton, 2012). A study of ovarian cancer cells found that tumor cells can reprogram normal stromal fibroblasts into CAFs by altering fibroblast expression of miRNAs, and these changes could be either duplicated or reversed in vitro by transfecting fibroblasts with the appropriate miRNAs and miRNA inhibitors (Mitra, 2012). So, targeting tumor stromal cells with miRNAs and miRNA inhibitors may be a valuable therapeutic strategy. Of note, implicit in both "the reciprocal-interactions CAF model" and the original therapy-engineering vision of this project is the expectation that greater insight into inflammation and regeneration-related mechanisms can help us manipulate cells and engineer effective cancer therapies in vitro. Over the course of evolution, the protection of vital stem-like cells has been a priority. Animals that can generate and protect critical (normal) stem-like cells in response to injury and inflammation have a survival advantage. But, for cancer patients, the unfortunate protection of lethal, stem-like tumor cells is a problem that needs to be reversed. 


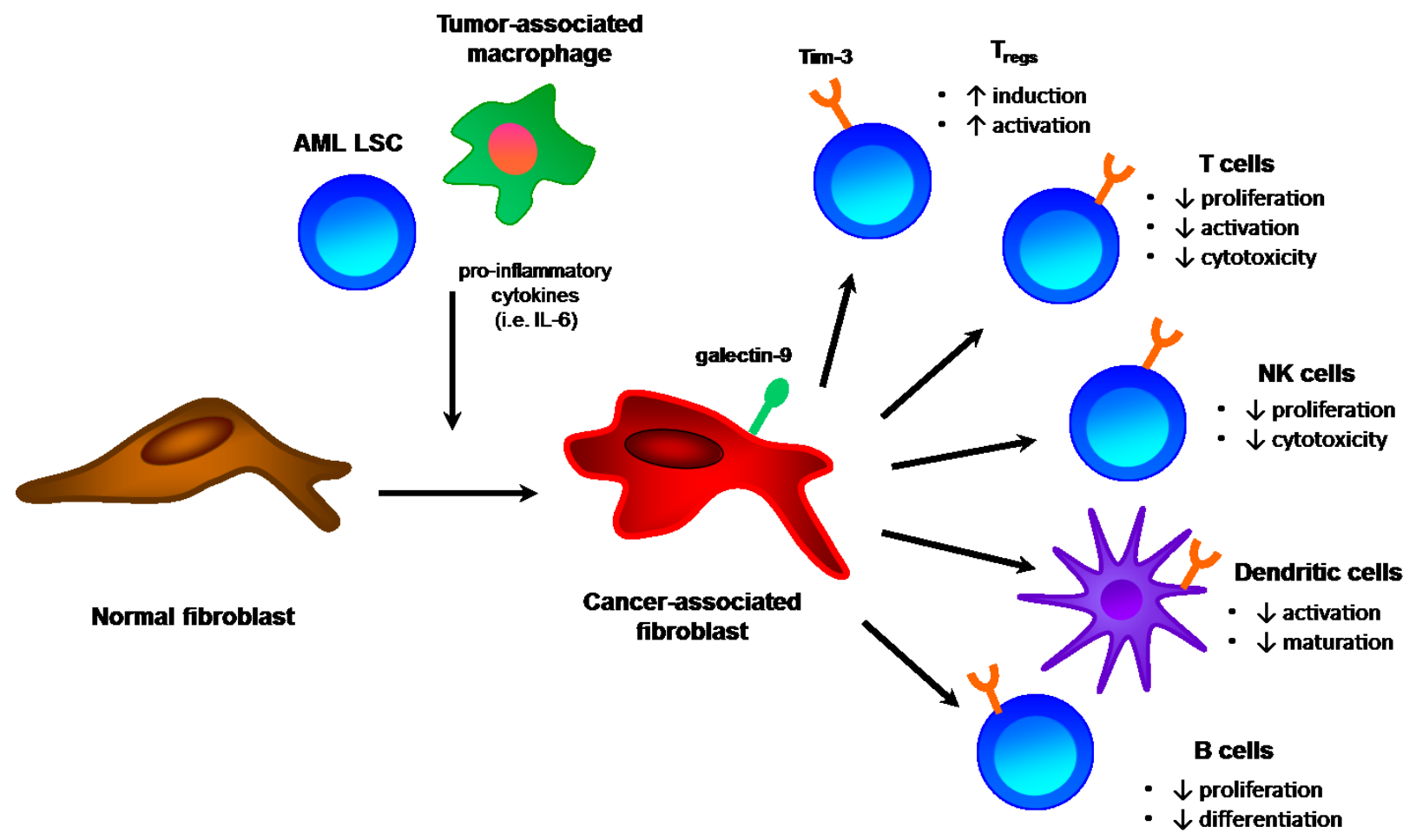

Figure 3.6. Role of AML-impaired stromal cells in immune suppression. A potential model for how AML-distorted CAFs suppress anti-tumor immunity is shown. Leukemia cells recruit immune cells such as tumor-associated macrophages which release proinflammatory cytokines (i.e. IL-6). The pro-inflammatory tumor microenvironment triggers conversion of bone marrow stromal cells into CAFs. CAFs may suppress infiltrating immune cells through production and release of immunosuppressive factors and enzymes as well as cell-to-cell contact (i.e. galectin-9-Tim-3 interactions). Adapted with permission from: Sioud, M. (2011). New insights into mesenchymal stromal cell-mediated T-cell suppression through galectins. Scandinavian Journal of Immunology, 73(2), 80. 
Figure 3.6 is a deliberately simplistic depiction of how CAFs may induce immunecell defects at the molecular level. IL-6 and Tim-3 are highlighted in this diagram because these two factors have been found to play key roles in AML disease (Sugiyama, 1996; Kikushige, 2010). Furthermore, the IL-6 secreted by AML cells may trigger generation of CAFs in cancer patients (Cirri, 2011). A more realistic model would acknowledge that interactions are likely to be bi-directional and feedback loops may act so quickly it may be difficult to identify cause-and-effect sequences. In addition to IL-6 and Tim-3, many other cytokines (like IFN-y) and antigens (like MUC-1) may promote immune-system dysfunction. Overexpression of the immune-checkpoint receptor Tim-3 (Kane, 2010) is used here as one potentially informative, and therapeutically relevant, example. Of note, Tim-3 is expressed not only by the malignant cells of diverse cancers, but is also expressed on the surfaces of microglial, mast, monocytic, macrophage, dendritic, and T (cytotoxic, helper, and regulatory) cells (Nakae, 2007; Wang, 2011). Tim-3 ligands include galectin-9 and phosphatidylserine (Zhu, 2005; DeKruyff, 2010).

Tim-3 is considered a critically important factor in AML pathogenesis because it is a surface antigen expressed by highly evolved AML LSCs (Kikushige, 2010). In general, tumor cells can evolve in response to immune-selection pressures, and coexpression of Tim-3 and PD1 increases, for example, as AML disease progresses (Zhou, 2011). Blocking both the PD1/PDL1 and Tim-3/galectin-9 axes can reduce AML tumor burden and mortality (Zhou, 2011). In mice with advanced AML, CD8 ${ }^{+} T$ cells co-expressing PD1 and Tim-3 synthesized less IFN- $\gamma$, TNF- $\alpha$, and IL-2 than T cells which did not express PD1 or Tim-3 when exposed to AML cells expressing the ligands PDL1 and galectin-9 (Tim-3 ligand) (Zhou, 2011). In galectin-9 knock-out mice, AML progressed more slowly 
and fewer $T_{\text {regs }}$ accumulated compared to wildtype mice (Zhou, 2011). In PD1 knock-out mice, Tim-3 was upregulated on T cells as AML progressed, and Tim-3-expressing CD8 ${ }^{+}$ T cells showed impaired cytokine secretion compared to wildtype mice (Zhou, 2011). Administering a Tim-3 blocking antibody to mice with human AML reduces their tumor burden and eliminates the primitive AML cells that can reconstitute AML in secondary recipients (Kikushige, 2010; Zhou, 2011; Kikushige, 2012).

In general, immunotherapy seeks to eliminate tumor cells - especially the relapsecausing CSCs - and overcome, or reverse, immune and/or stromal-cell defects that prevent effective anti-tumor immunity. Chapter IV reviews examples of promising anticancer immunotherapies, including adoptive transfer of a patient's ex vivo-modified antigen-presenting and immune-effector cells, antibodies, vaccines, and agents that modulate immune-checkpoint receptors such as CTLA-4 and Tim-3. These strategies are designed to target tumor cells, dysfunctional immune cells, and/or the immunosuppressive tumor microenvironment. Conventional therapies such as radiotherapy and chemotherapeutic agents that induce immunogenic tumor cell death (aka "cryptic vaccines") can also be considered immune therapies. Of course, the ultimate benefit of some therapies is the induction of a protective immunologic "memory" involving immune cells that prevent re-emergence of disease by providing constant tumor-cell surveillance. "Memory T cells," for example, have induced long-term remissions - perhaps lifelong cures - in some leukemia patients. 


\section{References}

Allavena, P., \& Mantovani, A. (2012). Immunology in the clinic review series; focus on cancer: tumour-associated macrophages: undisputed stars of the inflammatory tumour microenvironment. Clinical \& Experimental Immunology, 167(2), 195-205.

Alici, E. (2010). IPH-2101, a fully human anti-NK-cell inhibitory receptor mAb for the potential treatment of hematological cancers. Current Opinion in Molecular Therapeutics, 12(6), 724-733.

Anderson, A. C. (2012). Tim-3, a negative regulator of anti-tumor immunity. Current Opinion in Immunology, 24(2), 213-216.

Anton, K., Banerjee, D., \& Glod, J. (2012). Macrophage-associated mesenchymal stem cells assume an activated, migratory, pro-inflammatory phenotype with increased IL-6 and CXCL10 secretion. PloS One, 7(4), e35036.

Baitsch, L., Fuertes-Marraco, S. A., Legat, A., et al. (2012). The three main stumbling blocks for anticancer T cells. Trends in Immunology, 33(7), 364-372.

Barber, D. L., Wherry, E. J., Masopust, D., et al. (2005). Restoring function in exhausted CD8 T cells during chronic viral infection. Nature, 439(7077), 682-687.

Beatty, G. L., Chiorean, E. G., Fishman, M. P., et al. (2011). CD40 agonists alter tumor stroma and show efficacy against pancreatic carcinoma in mice and humans. Science, 331(6024), 1612-1616.

Biswas, S. K., \& Mantovani, A. (2010). Macrophage plasticity and interaction with lymphocyte subsets: cancer as a paradigm. Nature Immunology, 11(10), 889-896.

Borg, C., Terme, M., Taïeb, J., et al. (2004). Novel mode of action of c-kit tyrosine kinase inhibitors leading to NK cell-dependent antitumor effects. Journal of Clinical Investigation, 114(3), 379-388.

Boussiotis, V. A., Barber, D. L., Nakarai, T., et al. (1994). Prevention of T cell anergy by signaling through the gamma c chain of the IL-2 receptor. Science, 266(5187), 10391042.

Brahmer, J. R., Tykodi, S. S., Chow, L. Q., et al. (2012). Safety and activity of anti-PDL1 antibody in patients with advanced cancer. New England Journal of Medicine, 366(26), 2455-2465.

Bronte, V., \& Zanovello, P. (2005). Regulation of immune responses by L-arginine metabolism. Nature Reviews Immunology, 5(8), 641-654. 
Brown, I. E., Blank, C., Kline, J., et al. (2006). Homeostatic proliferation as an isolated variable reverses CD8+ $\mathrm{T}$ cell anergy and promotes tumor rejection. The Journal of Immunology, 177(7), 4521-4529.

Buhtoiarov, I. N., Lum, H. D., Berke, G., et al. (2006). Synergistic activation of macrophages via CD40 and TLR9 results in T cell independent antitumor effects. The Journal of Immunology, 176(1), 309-318.

Buhtoiarov, I. N., Sondel, P. M., Wigginton, J. M., et al. (2011). Anti-tumour synergy of cytotoxic chemotherapy and anti-CD40 plus CpG-ODN immunotherapy through repolarization of tumour-associated macrophages. Immunology, 132(2), 226-239.

Chappert, P., \& Schwartz, R. H. (2010). Induction of T cell anergy: integration of environmental cues and infectious tolerance. Current Opinion in Immunology, 22(5), 552559.

Cheng, F., Wang, H. W., Cuenca, A., et al. (2003). A critical role for Stat3 signaling in immune tolerance. Immunity, 19(3), 425-436.

Chiba, S., Baghdadi, M., Akiba, H., et al. (2012). Tumor-infiltrating DCs suppress nucleic acid-mediated innate immune responses through interactions between the receptor TIM3 and the alarmin HMGB1. Nature Immunology, 13(9), 832-842.

Cirri, P., \& Chiarugi, P. (2011). Cancer associated fibroblasts: the dark side of the coin. American Journal of Cancer Research, 1(4), 482.

Costello, R. T., Sivori, S., Marcenaro, E., et al. (2002). Defective expression and function of natural killer cell-triggering receptors in patients with acute myeloid leukemia. Blood, 99(10), 3661-3667.

Crespo, J., Sun, H., Welling, T. H., et al. (2013). T cell anergy, exhaustion, senescence, and stemness in the tumor microenvironment. Current Opinion in Immunology, 25(2), 214-221.

Danna, E. A., Sinha, P., Gilbert, M., et al. (2004). Surgical removal of primary tumor reverses tumor-induced immunosuppression despite the presence of metastatic disease. Cancer Research, 64(6), 2205-2211.

Das, S., Varalakshmi, C., \& Khar, A. (2000). Target-cell-induced anergy in natural killer cells: suppression of cytotoxic function. Cancer Immunology, Immunotherapy, 49(2), 109115.

da Silva, I. P., Gallois, A., Baranda, S. J., et al. (2014). Reversal of NK cell exhaustion in advanced melanoma by Tim-3 blockade. Cancer Immunology Research, 2(5), 410-422. 
DeKruyff, R. H., Bu, X., Ballesteros, A., et al. (2010). T cell/transmembrane, Ig, and mucin-3 allelic variants differentially recognize phosphatidylserine and mediate phagocytosis of apoptotic cells. The Journal of Immunology, 184(4), 1918-1930.

De Santo, C., Serafini, P., Marigo, I., et al. (2005). Nitroaspirin corrects immune dysfunction in tumor-bearing hosts and promotes tumor eradication by cancer vaccination. Proceedings of the National Academy of Sciences of the United States of America, 102(11), 4185-4190.

Dranoff, G. (2004). Cytokines in cancer pathogenesis and cancer therapy. Nature Reviews Cancer, 4(1), 11-22.

Dudek, A. M., Martin, S., Garg, A. D., et al. (2013). Immature, semi-mature, and fully mature dendritic cells: toward a DC-cancer cells interface that augments anticancer immunity. Frontiers in Immunology, 4(438), 1-14.

Dudley, M. E., Wunderlich, J. R., Robbins, P. F., et al. (2002). Cancer regression and autoimmunity in patients after clonal repopulation with antitumor lymphocytes. Science, 298(5594), 850-854.

Dunn, G. P., Bruce, A. T., Ikeda, H., et al. (2002). Cancer immunoediting: from immunosurveillance to tumor escape. Nature Immunology, 3(11), 991-998.

Dunn, G. P., Old, L. J., \& Schreiber, R. D. (2004). The three Es of cancer immunoediting. Annual Review of Immunology, 22, 329-360.

Dzivenu, O. K., O'Donnell-Tormey, J. (2003). Cancer and the immune system: the vital connection. Cancer Research Institute. Retrieved from:

http://www.cancerresearch.org/CRI/media/Content/Cancer\%20Immunotherapy/Cancerand-the-Immune-System-The-Vital-Connection.pdf

Frumento, G., Rotondo, R., Tonetti, M., et al. (2002). Tryptophan-derived catabolites are responsible for inhibition of $T$ and natural killer cell proliferation induced by indoleamine 2, 3-dioxygenase. The Journal of Experimental Medicine, 196(4), 459-468.

Gabrilovich, D. (2004). Mechanisms and functional significance of tumour-induced dendritic-cell defects. Nature Reviews Immunology, 4(12), 941-952.

Gabrilovich, D. I., \& Nagaraj, S. (2009). Myeloid-derived suppressor cells as regulators of the immune system. Nature Reviews Immunology, 9(3), 162-174.

Gajewski, T. F., Meng, Y., Blank, C., et al. (2006). Immune resistance orchestrated by the tumor microenvironment. Immunological Reviews, 213(1), 131-145. 
Ghiringhelli, F., Larmonier, N., Schmitt, E., et al. (2004). CD4+ CD25+ regulatory T cells suppress tumor immunity but are sensitive to cyclophosphamide which allows immunotherapy of established tumors to be curative. European Journal of Immunology, 34(2), 336-344.

Gieseke, F., Kruchen, A., Tzaribachev, N., et al. (2013). Proinflammatory stimuli induce galectin-9 in human mesenchymal stromal cells to suppress T-cell proliferation. European Journal of Immunology, 43(10), 2741-2749.

Goldberg, M. V., \& Drake, C. G. (2011). LAG-3 in cancer immunotherapy. Cancer Immunology and Immunotherapy (pp. 269-278). Springer Berlin Heidelberg.

Golden-Mason, L., Palmer, B. E., Kassam, N., et al. (2009). Negative immune regulator Tim-3 is overexpressed on T cells in hepatitis $C$ virus infection and its blockade rescues dysfunctional CD4+ and CD8+ T cells. Journal of Virology, 83(18), 9122-9130.

Gu, X. X. J., Yue, F. Y., Kovacs, C. M., et al. (2007). The role of cytokines which signal through the common $y$ chain cytokine receptor in the reversal of HIV specific CD4+ and CD8+ T cell anergy. PLoS One, 2(3), e300.

Guiducci, C., Vicari, A. P., Sangaletti, S., et al. (2005). Redirecting in vivo elicited tumor infiltrating macrophages and dendritic cells towards tumor rejection. Cancer Research, 65(8), 3437-3446.

Hastings, W. D., Anderson, D. E., Kassam, N., et al. (2009). TIM-3 is expressed on activated human CD4+ T cells and regulates Th1 and Th17 cytokines. European Journal of Immunology, 39(9), 2492-2501.

Haviv, I., Polyak, K., Qiu, W., et al. (2009). Origin of carcinoma associated fibroblasts. Cell Cycle, 8(4), 589-595.

Hodi, F. S., O'Day, S. J., McDermott, D. F., et al. (2010). Improved survival with ipilimumab in patients with metastatic melanoma. New England Journal of Medicine, 363(8), 711-723.

Hori, S., Nomura, T., \& Sakaguchi, S. (2003). Control of regulatory T cell development by the transcription factor Foxp3. Science, 299(5609), 1057-1061.

Huang, X., Bai, X., Cao, Y., et al. (2010). Lymphoma endothelium preferentially expresses Tim-3 and facilitates the progression of lymphoma by mediating immune evasion. The Journal of Experimental Medicine, 207(3), 505-520.

Igney, F. H., \& Krammer, P. H. (2002). Immune escape of tumors: apoptosis resistance and tumor counterattack. Journal of Leukocyte Biology, 71(6), 907-920. 
Infanger, D. W., Lynch, M. E., \& Fischbach, C. (2013). Engineered culture models for studies of tumor-microenvironment interactions. Annual Review of Biomedical Engineering, 15, 29-53.

Inoue, H., \& Tani, K. (2014). Multimodal immunogenic cancer cell death as a consequence of anticancer cytotoxic treatments. Cell Death \& Differentiation, 21(1), 3949.

Jajosky, A. N., Coad, J. E., Vos, J. A., et al. (2014). RepSox slows decay of CD34+ acute myeloid leukemia cells and decreases $\mathrm{T}$ cell immunoglobulin mucin-3 expression. Stem Cells Translational Medicine, 3(7), 836-848.

Jin, H. T., Anderson, A. C., Tan, W. G., et al. (2010). Cooperation of Tim-3 and PD-1 in CD8 T-cell exhaustion during chronic viral infection. Proceedings of the National Academy of Sciences, 107(33), 14733-14738.

Jinushi, M. (2012). Regulatory mechanisms of nucleic acid-mediated innate immune responses in the tumor microenvironment. Oncolmmunology, 1(9), 1632-1634.

Jones, R. B., Ndhlovu, L. C., Barbour, J. D., et al. (2008). Tim-3 expression defines a novel population of dysfunctional $\mathrm{T}$ cells with highly elevated frequencies in progressive HIV-1 infection. The Journal of Experimental Medicine, 205(12), 2763-2779.

Ju, Y., Hou, N., Zhang, X. N., et al. (2009). Blockade of Tim-3 pathway ameliorates interferon-gamma production from hepatic CD8+ T cells in a mouse model of hepatitis $B$ virus infection. Cellular and Molecular Immunology, 6(1), 35-43.

Kane, L. P. (2010). T cell Ig and mucin domain proteins and immunity. The Journal of Immunology, 184(6), 2743-2749.

Kerkar, S. P., \& Restifo, N. P. (2012). Cellular constituents of immune escape within the tumor microenvironment. Cancer Research, 72(13), 3125-3130.

Khaled, Y. S., Ammori, B. J., \& Elkord, E. (2013). Myeloid-derived suppressor cells in cancer: recent progress and prospects. Immunology and Cell Biology, 91(8), 493-502.

Kikushige, Y., \& Akashi, K. (2012). TIM-3 as a therapeutic target for malignant stem cells in acute myelogenous leukemia. Annals of the New York Academy of Sciences, 1266(1), 118-123.

Kikushige, Y., Shima, T., Takayanagi, S. I., et al. (2010). TIM-3 is a promising target to selectively kill acute myeloid leukemia stem cells. Cell Stem Cell, 7(6), 708-717.

Kim, J. S., Shin, D. C., Woo, M. Y., et al. (2012). T cell immunoglobulin mucin domain (TIM)-3 promoter activity in a human mast cell line. Immune Network, 12(5), 207-212. 
Kinjo, Y., Tupin, E., Wu, D., et al. (2006). Natural killer T cells recognize diacylglycerol antigens from pathogenic bacteria. Nature Immunology, 7(9), 978-986.

Kinjo, Y., Wu, D., Kim, G., et al. (2005). Recognition of bacterial glycosphingolipids by natural killer T cells. Nature, 434(7032), 520-525.

Kline, J., Brown, I. E., Zha, Y. Y., et al. (2008). Homeostatic proliferation plus regulatory T-cell depletion promotes potent rejection of B16 melanoma. Clinical Cancer Research, 14(10), 3156-3167.

Ko, K., Yamazaki, S., Nakamura, K., et al. (2005). Treatment of advanced tumors with agonistic anti-GITR mAb and its effects on tumor-infiltrating Foxp3+ CD25+ CD4+ regulatory T cells. The Journal of Experimental Medicine, 202(7), 885-891.

Kortylewski, M., Kujawski, M., Wang, T., et al. (2005). Inhibiting Stat3 signaling in the hematopoietic system elicits multicomponent antitumor immunity. Nature Medicine, 11(12), 1314-1321.

Kusmartsev, S., Cheng, F., Yu, B., et al. (2003). All-trans-retinoic acid eliminates immature myeloid cells from tumor-bearing mice and improves the effect of vaccination. Cancer Research, 63(15), 4441-4449.

Lathers, D. M., Clark, J. I., Achille, N. J., et al. (2004). Phase 1B study to improve immune responses in head and neck cancer patients using escalating doses of 25-hydroxyvitamin D3. Cancer Immunology, Immunotherapy, 53(5), 422-430.

Le Dieu, R., Taussig, D. C., Ramsay, A. G., et al. (2009). Peripheral blood T cells in acute myeloid leukemia (AML) patients at diagnosis have abnormal phenotype and genotype and form defective immune synapses with AML blasts. Blood, 114(18), 3909-3916.

Lion, E., Willemen, Y., Berneman, Z. N., et al. (2012). Natural killer cell immune escape in acute myeloid leukemia. Leukemia, 26(9), 2019-2026.

Liu, X., Shin, N., Koblish, H. K., et al. (2010). Selective inhibition of IDO1 effectively regulates mediators of antitumor immunity. Blood, 115(17), 3520-3530.

Liu, V. C., Wong, L. Y., Jang, T., et al. (2007). Tumor evasion of the immune system by converting CD4+ CD25- T cells into CD4+ CD25+ T regulatory cells: role of tumorderived TGF- $\beta$. The Journal of Immunology, 178(5), 2883-2892.

Lutsiak, M. C., Semnani, R. T., De Pascalis, R., et al. (2005). Inhibition of CD4+ 25+ T regulatory cell function implicated in enhanced immune response by low-dose cyclophosphamide. Blood, 105(7), 2862-2868. 
Lutz, M. B., \& Schuler, G. (2002). Immature, semi-mature and fully mature dendritic cells: which signals induce tolerance or immunity? Trends in Immunology, 23(9), 445-449.

Mattner, J., DeBord, K. L., Ismail, N., et al. (2005). Exogenous and endogenous glycolipid antigens activate NKT cells during microbial infections. Nature, 434(7032), 525-529.

Melani, C., Sangaletti, S., Barazzetta, F. M., et al. (2007). Amino-biphosphonatemediated MMP-9 inhibition breaks the tumor-bone marrow axis responsible for myeloidderived suppressor cell expansion and macrophage infiltration in tumor stroma. Cancer Research, 67(23), 11438-11446.

Mellman, I., Coukos, G., \& Dranoff, G. (2011). Cancer immunotherapy comes of age. Nature, 480(7378), 480-489.

Mengshol, J. A., Golden-Mason, L., Arikawa, T., et al. (2010). A crucial role for Kupffer cell-derived galectin-9 in regulation of T cell immunity in hepatitis C infection. PLoS One, 5(3), e9504.

Mitra, A. K., Zillhardt, M., Hua, Y., et al. (2012). MicroRNAs reprogram normal fibroblasts into cancer-associated fibroblasts in ovarian cancer. Cancer Discovery, 2(12), 11001108.

Mirza, N., Fishman, M., Fricke, I., et al. (2006). All-trans-retinoic acid improves differentiation of myeloid cells and immune response in cancer patients. Cancer Research, 66(18), 9299-9307.

Munn, D. H., Sharma, M. D., Lee, J. R., et al. (2002). Potential regulatory function of human dendritic cells expressing indoleamine 2, 3-dioxygenase. Science, 297(5588), 1867-1870.

Nakae, S., likura, M., Suto, H., et al. (2007). TIM-1 and TIM-3 enhancement of Th2 cytokine production by mast cells. Blood, 110(7), 2565-2568.

Ndhlovu, L. C., Lopez-Vergès, S., Barbour, J. D., et al. (2012). Tim-3 marks human natural killer cell maturation and suppresses cell-mediated cytotoxicity. Blood, 119(16), 3734-3743.

Östman, A., \& Augsten, M. (2009). Cancer-associated fibroblasts and tumor growthbystanders turning into key players. Current Opinion in Genetics \& Development, 19(1), 67-73.

Ostrand-Rosenberg, S., \& Sinha, P. (2009). Myeloid-derived suppressor cells: linking inflammation and cancer. The Journal of Immunology, 182(8), 4499-4506. 
Oviedo, N. J., \& Beane, W. S. (2009). Regeneration: The origin of cancer or a possible cure? Seminars in Cell \& Developmental Biology, 20(5), 557-564.

Pan, P. Y., Wang, G. X., Yin, B., et al. (2008). Reversion of immune tolerance in advanced malignancy: modulation of myeloid-derived suppressor cell development by blockade of stem-cell factor function. Blood, 111(1), 219-228.

Pardoll, D. M. (2012). The blockade of immune checkpoints in cancer immunotherapy. Nature Reviews Cancer, 12(4), 252-264.

Pinzon-Charry, A., Maxwell, T., \& Lopez, J. A. (2005). Dendritic cell dysfunction in cancer: a mechanism for immunosuppression. Immunology and Cell Biology, 83(5), 451-461.

Raber, P., Ochoa, A. C., \& Rodríguez, P. C. (2012). Metabolism of L-arginine by myeloidderived suppressor cells in cancer: mechanisms of $\mathrm{T}$ cell suppression and therapeutic perspectives. Immunological Investigations, 41(6-7), 614-634.

Raval, R. R., Sharabi, A. B., Walker, A. J., et al. (2014). Tumor immunology and cancer immunotherapy: summary of the 2013 SITC primer. Journal for ImmunoTherapy of Cancer, 2, 14.

Rech, A. J., \& Vonderheide, R. H. (2009). Clinical use of anti-CD25 antibody daclizumab to enhance immune responses to tumor antigen vaccination by targeting regulatory $T$ cells. Annals of the New York Academy of Sciences, 1174(1), 99-106.

Rech, A. J., Mick, R., Martin, S., et al. (2012). CD25 blockade depletes and selectively reprograms regulatory $T$ cells in concert with immunotherapy in cancer patients. Science Translational Medicine, 4(134), 134ra62.

Richards, J. O., Chang, X., Blaser, B. W., et al. (2006). Tumor growth impedes naturalkiller-cell maturation in the bone marrow. Blood, 108(1), 246-252.

Rolny, C., Mazzone, M., Tugues, S., et al. (2011). HRG inhibits tumor growth and metastasis by inducing macrophage polarization and vessel normalization through downregulation of PIGF. Cancer Cell, 19(1), 31-44.

Romagné, F., André, P., Spee, P., et al. (2009). Preclinical characterization of 1-7F9, a novel human anti-KIR receptor therapeutic antibody that augments natural killermediated killing of tumor cells. Blood, 114(13), 2667-2677.

Rosenberg, S. A., Restifo, N. P., Yang, J. C., Morgan, R. A., \& Dudley, M. E. (2008). Adoptive cell transfer: a clinical path to effective cancer immunotherapy. Nature Reviews Cancer, 8(4), 299-308. 
Sabatos, C. A., Chakravarti, S., Cha, E., et al. (2003). Interaction of Tim-3 and Tim-3 ligand regulates $\mathrm{T}$ helper type 1 responses and induction of peripheral tolerance. Nature Immunology, 4(11), 1102-1110.

Sada-Ovalle, I., Chávez-Galán, L., Torre-Bouscoulet, L., et al. (2012). The Tim3-galectin 9 pathway induces antibacterial activity in human macrophages infected with mycobacterium tuberculosis. The Journal of Immunology, 189(12), 5896-5902.

Sakhdari, A., Mujib, S., Vali, B., et al. (2012). Tim-3 negatively regulates cytotoxicity in exhausted CD8+ T cells in HIV infection. PloS One, 7(7), e40146.

Sakuishi, K., Apetoh, L., Sullivan, J. M., et al. (2010). Targeting Tim-3 and PD-1 pathways to reverse $\mathrm{T}$ cell exhaustion and restore anti-tumor immunity. The Journal of Experimental Medicine, 207(10), 2187-2194.

Sakuishi, K., Ngiow, S. F., Sullivan, J., et al. (2013). Tim-3+ FoxP3+ regulatory T cells are tissue specific promoters of T cell dysfunction in cancer. Oncoimmunology, 2(4), e23849.

Sánchez-Fueyo, A., Tian, J., Picarella, D., et al. (2003). Tim-3 inhibits T helper type 1mediated auto-and alloimmune responses and promotes immunological tolerance. Nature Immunology, 4(11), 1093-1101.

Seki, M., Oomizu, S., Sakata, K. M., et al. (2008). Galectin-9 suppresses the generation of Th17, promotes the induction of regulatory $\mathrm{T}$ cells, and regulates experimental autoimmune arthritis. Clinical Immunology, 127(1), 78-88.

Serafini, P., Meckel, K., Kelso, M., et al. (2006). Phosphodiesterase-5 inhibition augments endogenous antitumor immunity by reducing myeloid-derived suppressor cell function. The Journal of Experimental Medicine, 203(12), 2691-2702.

Shimizu, J., Yamazaki, S., \& Sakaguchi, S. (1999). Induction of tumor immunity by removing CD25+ CD4+ T cells: a common basis between tumor immunity and autoimmunity. The Journal of Immunology, 163(10), 5211-5218.

Sica, A., \& Bronte, V. (2007). Altered macrophage differentiation and immune dysfunction in tumor development. Journal of Clinical Investigation, 117(5), 1155-1166.

Sioud, M. (2011). New insights into mesenchymal stromal cell-mediated T-Cell suppression through galectins. Scandinavian Journal of Immunology, 73(2), 79-84.

Solinas, G., Germano, G., Mantovani, A., et al. (2009). Tumor-associated macrophages (TAM) as major players of the cancer-related inflammation. Journal of Leukocyte Biology, 86(5), 1065-1073. 
Spranger, S., \& Gajewski, T. (2013). Rational combinations of immunotherapeutics that target discrete pathways. Journal for ImmunoTherapy of Cancer, 1, 1-14.

Sugiyama, H., Inoue, K., Ogawa, H., et al. (1996). The expression of IL-6 and its related genes in acute leukemia. Leukemia \& Lymphoma, 21(1-2), 49-52.

Suzuki, E., Kapoor, V., Jassar, A. S., et al. (2005). Gemcitabine selectively eliminates splenic Gr-1+/CD11b+ myeloid suppressor cells in tumor-bearing animals and enhances antitumor immune activity. Clinical Cancer Research, 11(18), 6713-6721.

Szczepanski, M. J., Szajnik, M., Welsh, A., et al. (2010). Interleukin-15 enhances natural killer cell cytotoxicity in patients with acute myeloid leukemia by upregulating the activating NK cell receptors. Cancer Immunology, Immunotherapy, 59(1), 73-79.

Tang, X. (2013). Tumor-associated macrophages as potential diagnostic and prognostic biomarkers in breast cancer. Cancer Letters, 332(1), 3-10.

Tang, X., Mo, C., Wang, Y., et al. (2013). Anti-tumour strategies aiming to target tumourassociated macrophages. Immunology, 138(2), 93-104.

Teague, R. M., Sather, B. D., Sacks, J. A., et al. (2006). Interleukin-15 rescues tolerant CD8+ T cells for use in adoptive immunotherapy of established tumors. Nature Medicine, 12(3), 335-341.

Telang, S., Rasku, M., Clem, A., et al. (2011). Phase II trial of the regulatory T celldepleting agent, denileukin diftitox, in patients with unresectable stage IV melanoma. BMC Cancer, 11(1), 515.

Tesniere, A., Panaretakis, T., Kepp, O., et al. (2008). Molecular characteristics of immunogenic cancer cell death. Cell Death \& Differentiation, 15(1), 3-12.

Themeli, M., Kloss, C. C., Ciriello, G., et al. (2013). Generation of tumor-targeted human $\mathrm{T}$ lymphocytes from induced pluripotent stem cells for cancer therapy. Nature Biotechnology, 31(10), 928-933.

Topalian, S. L., Hodi, F. S., Brahmer, J. R., et al. (2012). Safety, activity, and immune correlates of anti-PD-1 antibody in cancer. New England Journal of Medicine, 366(26), 2443-2454.

Tuma, R. A., Giannino, R., Guirnalda, P., et al. (2002). Rescue of CD8 T cell-mediated antimicrobial immunity with a nonspecific inflammatory stimulus. Journal of Clinical Investigation, 110(10), 1493-1501. 
Uyttenhove, C., Pilotte, L., Théate, I., et al. (2003). Evidence for a tumoral immune resistance mechanism based on tryptophan degradation by indoleamine 2, 3dioxygenase.Nature Medicine, 9(10), 1269-1274.

van den Boorn, J. G., \& Hartmann, G. (2013). Turning tumors into vaccines: co-opting the innate immune system. Immunity, 39(1), 27-37.

Velardi, A., Ruggeri, L., Mancusi, A., et al. (2009). Natural killer cell allorecognition of missing self in allogeneic hematopoietic transplantation: a tool for immunotherapy of leukemia. Current Opinion in Immunology, 21(5), 525-530.

Vesely, M. D., Kershaw, M. H., Schreiber, R. D., et al. (2011). Natural innate and adaptive immunity to cancer. Annual Review of Immunology, 29, 235-271.

Vignali, D. A., Collison, L. W., \& Workman, C. J. (2008). How regulatory T cells work. Nature Reviews Immunology, 8(7), 523-532.

Vizcardo, R., Masuda, K., Yamada, D., et al. (2013). Regeneration of human tumor antigen-specific T cells from iPSCs derived from mature CD8+ T cells. Cell Stem Cell, 12(1), 31-36.

Wang, F., Xu, J., Liao, Y., et al. (2011). Tim-3 ligand galectin-9 reduces IL-17 level and accelerates Klebsiella pneumoniae infection. Cellular Immunology, 269(1), 22-28.

Watkins, S. K., Egilmez, N. K., Suttles, J., et al. (2007). IL-12 rapidly alters the functional profile of tumor-associated and tumor-infiltrating macrophages in vitro and in vivo. The Journal of Immunology, 178(3), 1357-1362.

Weinberg, A. D., Morris, N. P., Kovacsovics-Bankowski, M., et al. (2011). Science gone translational: the OX40 agonist story. Immunological Reviews, 244(1), 218-231.

Wherry, E. J. (2011). T cell exhaustion. Nature Immunology, 12(6), 492-499.

Wiener, Z., Kohalmi, B., Pocza, P., et al. (2006). TIM-3 is expressed in melanoma cells and is upregulated in TGF-beta stimulated mast cells. Journal of Investigative Dermatology, 127(4), 906-914.

Yang, Z. Z., Grote, D. M., Ziesmer, S. C., et al. (2012). IL-12 upregulates TIM-3 expression and induces $T$ cell exhaustion in patients with follicular $B$ cell non-Hodgkin lymphoma. The Journal of Clinical Investigation, 122(4), 1271.

Yang, X., Jiang, X., Chen, G., et al. (2013). T cell Ig mucin-3 promotes homeostasis of sepsis by negatively regulating the TLR response. The Journal of Immunology, 190(5), 2068-2079. 
Yao, S., Zhu, Y., \& Chen, L. (2013). Advances in targeting cell surface signalling molecules for immune modulation. Nature Reviews Drug Discovery, 12(2), 130-146.

Zhang, L., Chen, X., Liu, X., et al. (2013). CD40 ligation reverses T cell tolerance in acute myeloid leukemia. The Journal of Clinical Investigation, 123(5), 1999-2010.

Zhang, Y., Ma, C. J., Wang, J. M., et al. (2012). Tim-3 regulates pro-and anti-inflammatory cytokine expression in human CD14+ monocytes. Journal of Leukocyte Biology, 91(2), 189-196.

Zhou, Q., Munger, M. E., Veenstra, R. G., et al. (2011). Coexpression of Tim-3 and PD-1 identifies a CD8+ T-cell exhaustion phenotype in mice with disseminated acute myelogenous leukemia. Blood, 117(17), 4501-4510.

Zhu, C., Anderson, A. C., Schubart, A., et al. (2005). The Tim-3 ligand galectin-9 negatively regulates $T$ helper type 1 immunity. Nature Immunology, 6(12), 1245-1252.

Zitvogel, L., Apetoh, L., Ghiringhelli, F., et al. (2008). The anticancer immune response: indispensable for therapeutic success? The Journal of Clinical Investigation, 118(6), 1991-2001.

Zitvogel, L., \& Kroemer, G. (2012). Targeting PD-1/PD-L1 interactions for cancer immunotherapy. Oncoimmunology, 1(8), 1223-1225.

Zitvogel, L., Tesniere, A., \& Kroemer, G. (2006). Cancer despite immunosurveillance: immunoselection and immunosubversion. Nature Reviews Immunology, 6(10), 715-727.

Zou, W. (2006). Regulatory T cells, tumour immunity and immunotherapy. Nature Reviews Immunology, 6(4), 295-307. 


\section{Chapter IV}

Anti-cancer immunotherapy: strategies and recent successes 
Cancer immunotherapy is now a broad and rapidly expanding field (Raval, 2014). This chapter highlights recent technical advances and clinical successes achieved by new patient-specific and FDA-approved anti-cancer therapies, reviews AML immunotherapies in preclinical development, and discusses rational therapeutic combinations. Key features of effective anti-tumor immune responses involving innate and adaptive immune defenses are reviewed in Chapter III. Review articles are recommended that comprehensively address topics that are only briefly summarized in this dissertation.

\section{Introduction}

To save some cancer patients, combination strategies may be required that include immunologic components that can overcome cancer-cell immune-evasion and deathresistance, activate and repair immune cells, and neutralize immuno-suppressive factors in the tumor microenvironment (see Figure 4.1). Chapter II reviews strategies that increase the immunogenicity of cancer cells (making them more susceptible to immunologic attack), improve the presentation of leukemic antigens, overcome cancerinduced and immune-mediated death-resistance of cancer cells, and induce immunogenic tumor-cell death (ICD). Immunotherapies should (1) unleash pre-existing, tumor-reactive immune cells that are suppressed and/or (2) trigger de novo anti-tumor immune responses. Strategies to restore anti-tumor immune cells that have been silenced include removing the inhibitory "brakes" on exhausted immune cells (to restore their function) by blocking immune-checkpoint receptors like PD1 (Pardoll, 2012; Yao, 2013). Strategies to trigger de novo immune responses include inducing immunogenic tumor-cell death which can be considered a form of "vaccination in situ" (Tesniere, 2008; Inoue, 2014). Immunogenic - as opposed to tolerogenic - forms of cell death can release 
new, previously unexposed leukemic antigens or present previously non-immunogenic antigens in a new, immune-stimulatory context that is enhanced by "danger" signals. ICD can trigger antigen-presenting cells (APCs) to uptake and cross-present leukemia-cell antigens to $T$ cells and, thereby, stimulate anti-tumor immunity. Ideally, therapies should stimulate both the activation and effector phases of the innate and adaptive immune cascades, including both the cellular and humoral arms (Yao, 2013). While the activation phase of adaptive immune responses involves tumor antigen cross-presentation to $T$ cells in lymphoid organs, the effector phase involves tumor-cell lysis in the periphery. Chapter III reviews strategies to repair and activate antigen-presenting and immuneeffector cells as well as eliminate immuno-suppressive cells in the tumor microenvironment. This chapter reviews cancer immunotherapies involving antibodies, vaccines, adoptive transfer of immune cells, and leukemia-derived APCs. These strategies can be combined with approaches that enhance immune responses in nonspecific ways such as immunogenic chemotherapies (Obeid, 2007; Zitvogel, 2008; Vanneman, 2012). 


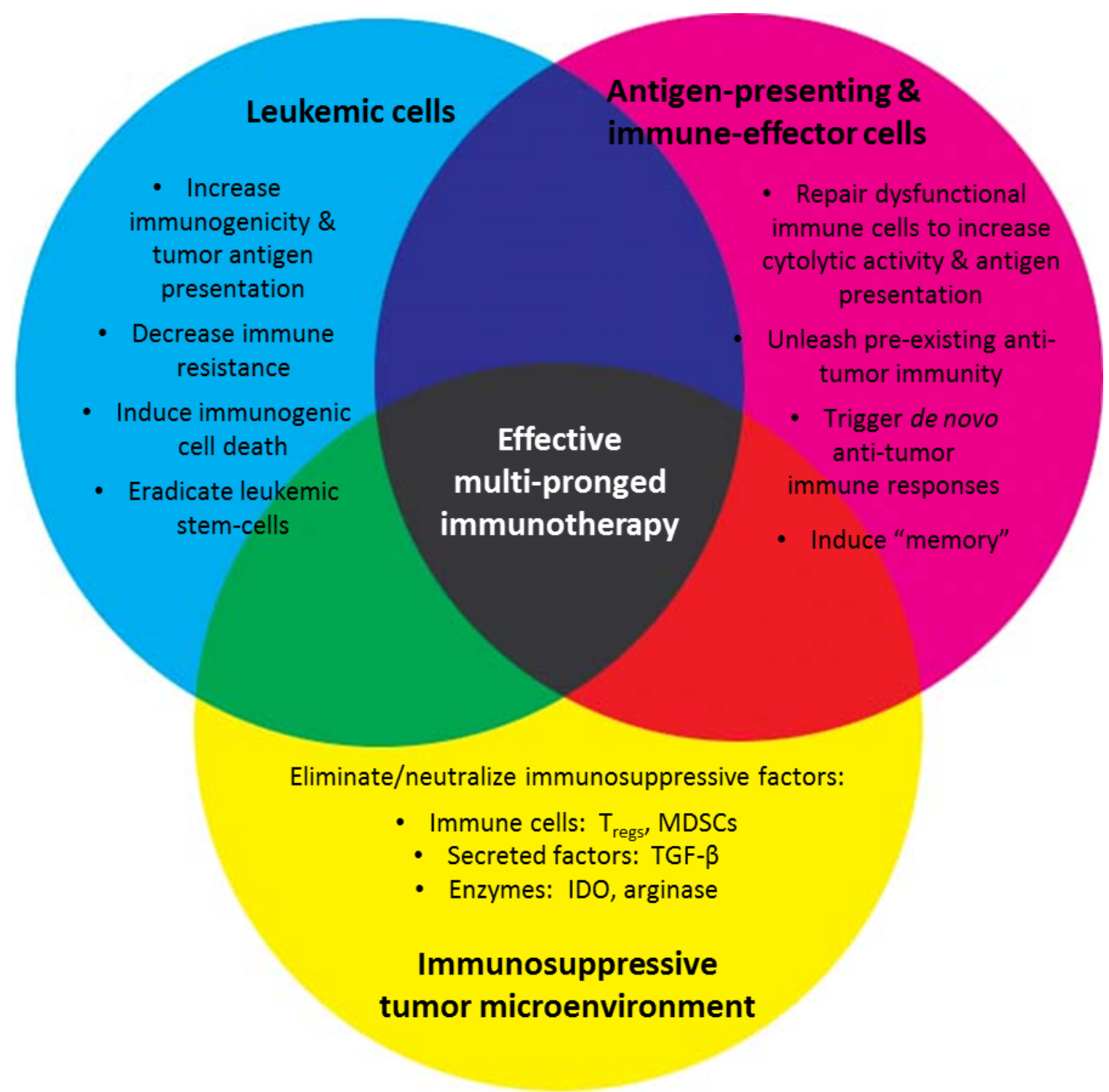

Figure 4.1. Three-pronged anti-cancer immunotherapeutic strategy. To achieve lasting anti-tumor immunity, targeting of (i) tumor cells, (ii) the immune system, and (iii) the immunosuppressive tumor microenvironment may be required. Tumor cells may need to be altered to increase antigen presentation and decrease death resistance. Dysfunctional antigen-presenting and immune-effector cells may need to be repaired to become activated, responsive, and functional. Finally, the tumor microenvironment may need to be targeted to neutralize immunosuppressive factors and eliminate immunosuppressive cell types. 


\section{Anti-cancer immunotherapeutic strategies}

\section{Adoptive transfer of immune-effector cells}

Studies of allogeneic hematopoietic stem cell transplantation (HSCT) show that donor T and NK cells can induce potent graft-versus-leukemia (GvL) immune responses that have cured some patients (Ruggeri, 2002; Bleakley, 2004). However, HSCT carries the risk of graft-versus host disease (GvHD). Using a patient's own NK or T cells ("autologous" cells) eliminates the risk of immune incompatibilities. Recently, cancer patients have experienced dramatic remissions after being treated with their own cancerreactive, tumor-infiltrating T cells that were isolated, expanded ex vivo, and re-infused as therapy (Tran, 2014). Alternatively, a patient's NK or T cells can be genetically engineered to express receptors that target (bind) antigens on the patient's tumor cells (Kalos, 2011; Porter, 2011). Immune cells to be used as therapy may also be genetically modified in other ways to increase their survival, proliferation, trafficking toward malignant cells, activity, and persistence (resistance to immune suppression) (Kershaw, 2013).

CAR-modified T cells: Of historic significance, adoptive transfer of autologous chimeric antigen receptor (CAR)-modified T cells induced rapid and sustained remissions in patients with advanced B cell leukemias (Brentjens, 2011; Brentjens, 2013; Kalos, 2011; Kockenderfer, 2012; Porter, 2011) and lymphomas (Kockenderfer, 2012). In a clinical trial, each chronic lymphocytic leukemia (CLL) patient's T cells were genetically engineered to express "second-generation" CARs composed of an extracellular CD19targeting domain fused with intracellular CD137 costimulatory and T-cell receptor $\zeta$ domains (Kalos, 2011; Porter, 2011). The antibody-binding domain directly recognizes antigens on the tumor-cell surface rather than peptide-MHC complexes, enabling T-cell 
activation without the need for antigen-presentation (Gross, 1989; Irving, 1991). Within CLL patients, CAR-modified T cells survived, persisted, killed $\sim 1,000$ cancer cells per effector, induced remission (>2 years now), and generated memory T cells (Kalos, 2011; Porter, 2011). Of note, tumor-cell death was so extensive, patients experienced "tumor lysis syndrome" and "cytokine release syndrome" (readily reversed using tocilizumab to block IL-6) along with aplasias of normal B-cells which were managed via immunoglobulin injections (Kalos, 2011; Porter, 2011).

$T$ cells "rejuvenated" through iPSC reprogramming: In patients with cancer (Vizcardo, 2013) and chronic HIV infection (Nishimura, 2013), reprogramming their exhausted $T$ cells to pluripotency - followed by re-differentiation back to $T$ cells rejuvenated their cytokine-secreting and cytolytic activities. "Rejuvenated" $\mathrm{T}$ cells retained their antigen-specificity and acquired high proliferative potential accompanied by elongated telomeres (Nishimura, 2013). Large numbers of functionally "rejuvenated" T cells may be further modified (via genetic engineering, for example) to express CARs prior to use as therapy (Themeli, 2013). Although using a patient's own (autologous) immune cells as therapy eliminates the risk of immune-incompatibilities (such as host rejection of foreign cells or GvHD), sufficient numbers of immune cells may not be available in severely immuno-compromised cancer patients. Therefore, researchers are exploring ways to generate large numbers of reprogrammed tumor-reactive $T$ cells that are universally tolerated by disrupting T-cell receptors or repressing human leukocyte antigens (HLA) (Themeli, 2013). Of note, reprogrammed T cells have not yet been evaluated in clinical trials. 


\section{LSC-targeted antibodies}

Treatment with antibodies directed against the antigens on AML LSCs (including antigens that emerge on highly evolved AML LSCs) - such as Tim-3 (Kikushige, 2010), CD47 (Majeti, 2009), CD44 (Jin, 2006), and interleukin (IL)-3 receptor a (IL3Ra or CD123) (Jordan, 2000; Jin, 2009) - has eliminated disease and extended survival in mice engrafted with AML. By promoting tumor-cell immune-escape (Jaiswal, 2009), several of these receptors may contribute to the poor immunogenicity of AML LSCs (Costello, 2000). For example, AML LSCs express CD47, a phagocyte "do not eat me" signal (Jaiswal, 2009), and CD47-targeting antibodies promote eradication of AML LSCs by facilitating their uptake and clearance by macrophages (Majeti, 2009). Gemtuzumab ozogamicin (Mylotarg), an anti-CD33 antibody fused to the toxin calicheamicin, is FDA-approved for AML patients with relapsed $\mathrm{CD}_{3} 3^{+}$disease (Larson, 2002). Antibodies may facilitate tumor-cell clearance by antibody-dependent cytotoxicity mediated by NK cells or by activation of the complement cascade. To enhance tumor-cell killing, antibodies may be conjugated with drugs, radioisotopes, cytokines, or toxins. Fortunately, leukemia-cell death, in turn, can stimulate anti-tumor immune responses by enhancing the uptake of leukemia antigens and their presentation to immune cells. 


\section{Immune-modulating antibodies}

Various co-stimulatory and co-inhibitory immune-checkpoint receptors are expressed by T cells and interact with their ligands on APCs and cancer cells (see Figure 4.2). Both (1) agonistic antibodies that activate costimulatory receptors such as CD28 and CD137/4-1BB (Melero, 2007) and (2) antagonistic antibodies that block critical coinhibitory receptors - such as Tim-3, programmed cell death protein 1 (PD-1), and cytotoxic T-lymphocyte-associated antigen 4 (CTLA-4) (Pardoll, 2012) - promote antitumor immunity (see Figure 4.3). Depending on the ligand and cell type(s) targeted, immune-modulating antibodies increase the cross-presentation of tumor antigens by DCs or other APCs in the lymph nodes and/or the killing of tumor cells by immune-effector (i.e. NK, T) cells in the peripheral tissues (see Figure 4.3). Of note, ipilimumab - an antibody that blocks CTLA-4 - is the first agent to ever improve the survival of patients with metastatic melanoma (Hodi, 2010). Adverse side effects of anti-CTLA-4 therapy include autoimmune reactions resulting from potent activation of not just tumor-reactive $T$ cells but self-reactive T cells as well (Hodi, 2010). CTLA-4 knock-out mice also suffer from severe autoimmune reactions (Tivol, 1995; Waterhouse, 1995). Co-blockade of the coinhibitory receptors Tim-3 and PD-1 improves anti-tumor immunity in mouse models of melanoma (Fourcade, 2010), supporting the rationale for combination therapies. As with traditional cancer therapies, immuno-modulating strategies can induce both beneficial and undesirable effects that must be carefully considered and anticipated. 


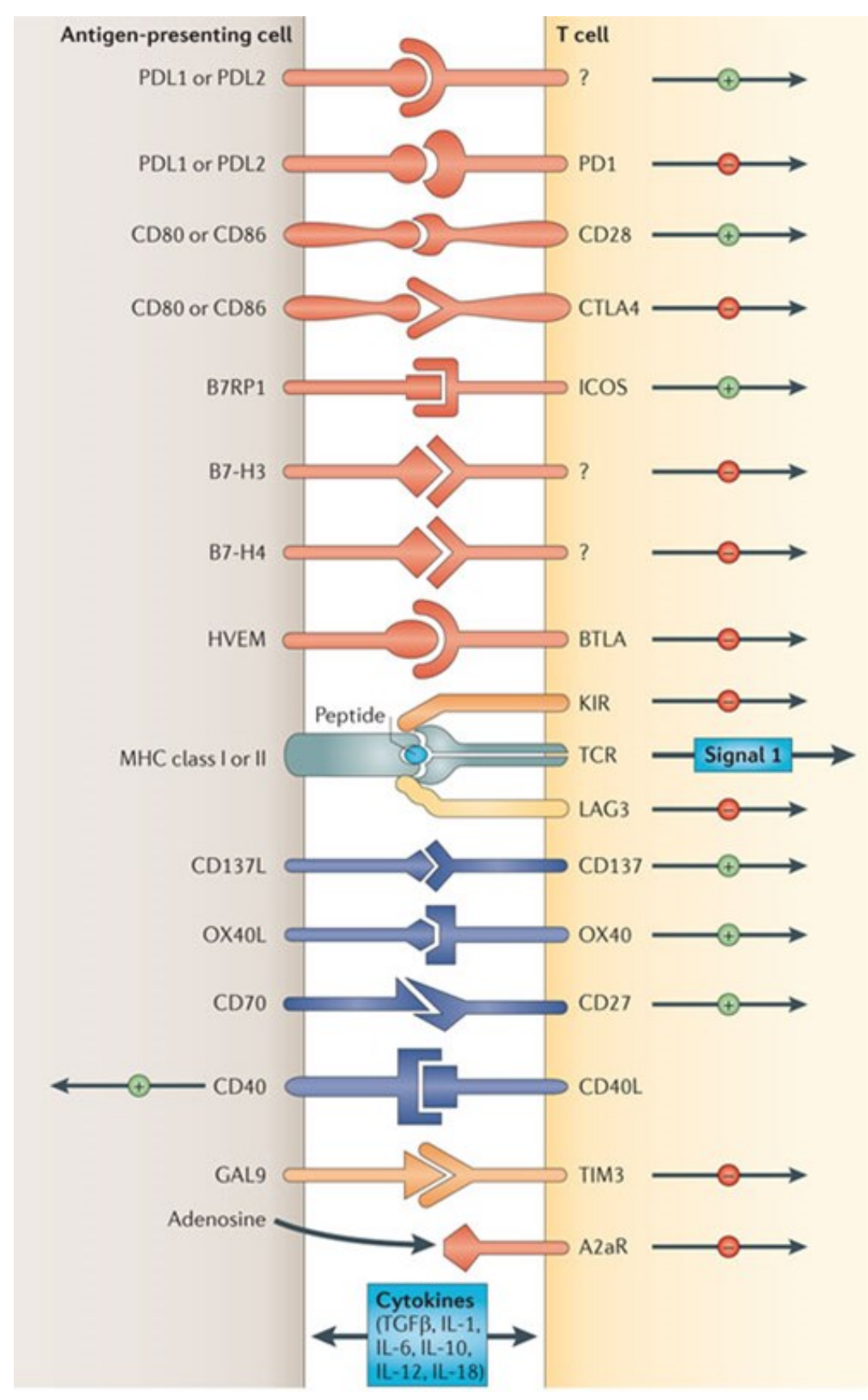

Figure 4.2. Immune checkpoint receptors and their ligands. Immunomodulatory cell surface receptors are emerging as important therapeutic targets for controlling cancer. Numerous co-inhibitory and co-stimulatory ligand-receptor interactions are known. The net effect on immune cell activation or suppression involves integration of all signals. Not only $T$ cells, but also cells of the innate immune system and tumor cells can express immune checkpoint receptors (i.e. Tim-3) as well as their ligands (i.e. PD1-ligand). Reproduced with permission from: Pardoll, D. M. (2012). The blockade of immune checkpoints in cancer immunotherapy. Nature Reviews Cancer, 12(4), 254. 
a Lymphoid organs: modulation of priming phase

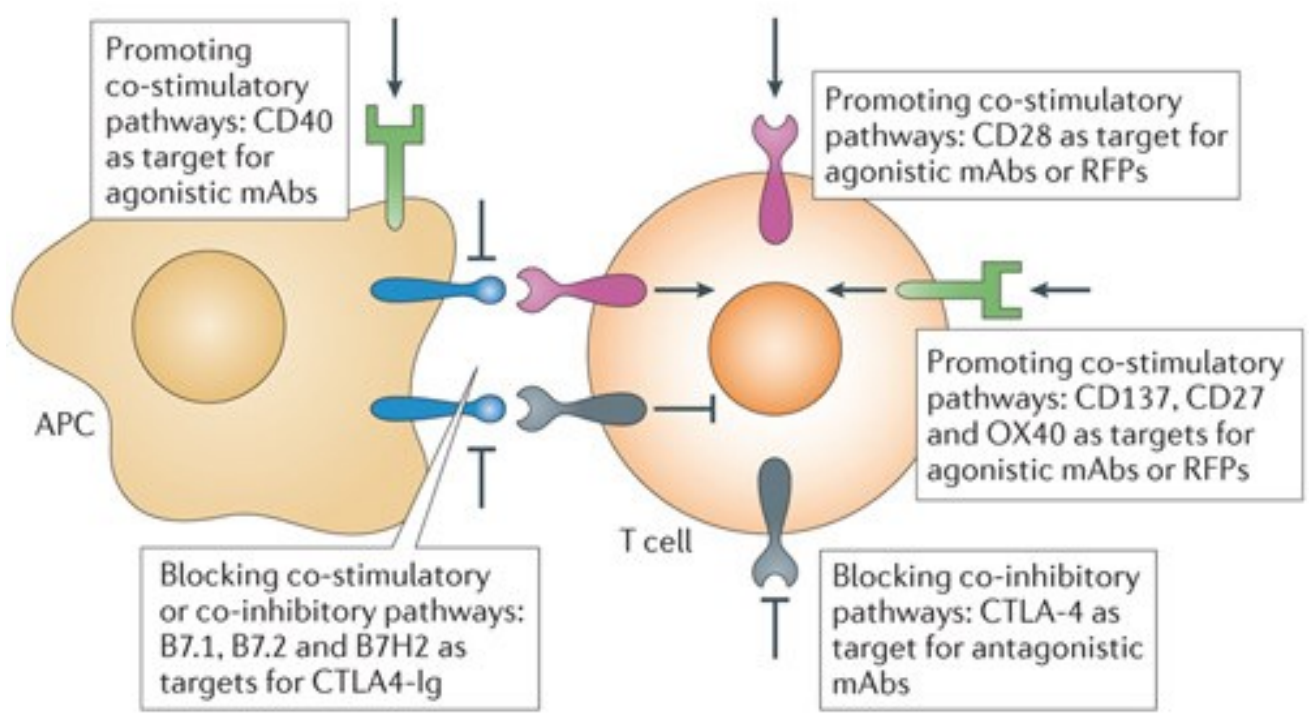

b Peripheral tissues: modulation of effector phase

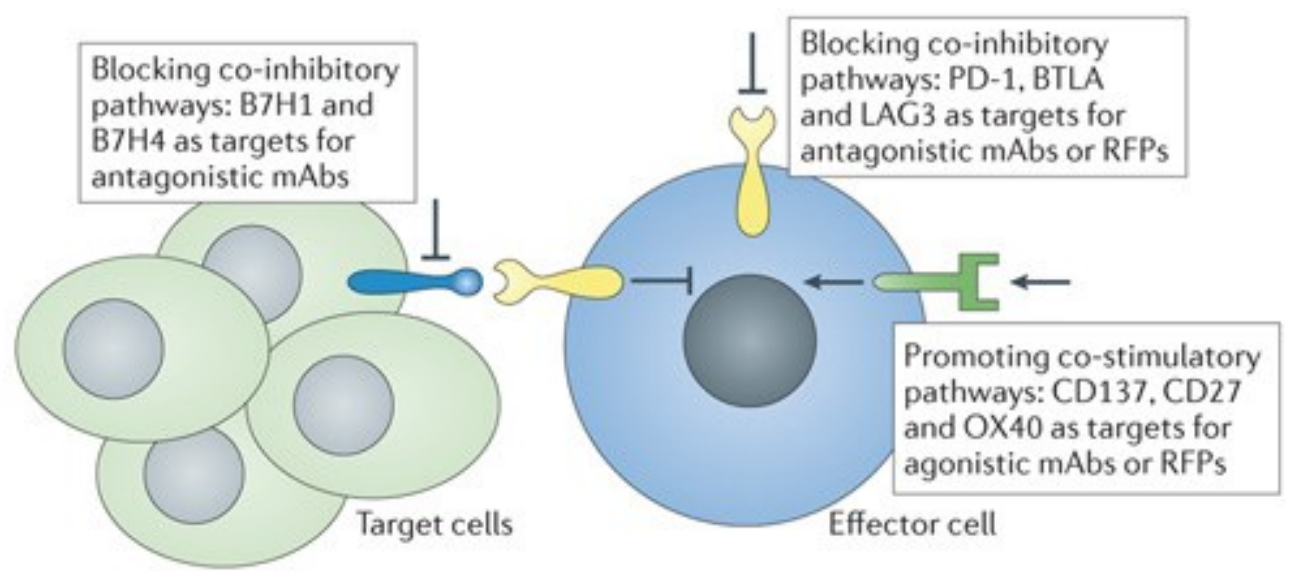

Figure 4.3. Targeting immunomodulatory receptors to improve anti-tumor immunity. Agonistic antibodies to co-stimulatory receptors and antagonistic (blocking) antibodies to co-inhibitory receptors can be used therapeutically to amplify host immune responses against tumors. Potential areas of therapeutic interventions include: ligandreceptor interactions between antigen-presenting cells (i.e. DCs) and T cells within lymph nodes during the "priming phase" (a) and ligand-receptor interactions between immune effector cells and tumor cell targets in the periphery during the "effector phase" (b). Reproduced with permission from: Yao, S., Zhu, Y., \& Chen, L. (2013). Advances in targeting cell surface signalling molecules for immune modulation. Nature Reviews Drug Discovery, 12(2), 135. 


\section{Vaccines}

Therapeutic cancer vaccines - used to treat patients after cancer is diagnosed may include whole tumor cells, tumor peptides, APCs, viruses, and immune-stimulating adjuvants (Schlom, 2012). Anti-leukemia cell-based vaccines include inactivated whole tumor cells that may be modified ex vivo to improve their antigen-presenting or immunostimulating capacity (Burkhardt, 2013). Utilizing whole tumor cells (as opposed to a limited number of peptides) may trigger immune responses against multiple leukemiaassociated antigens, reduce the likelihood of selecting for "escape variants," and eliminate the need to identify patient-specific immunogenic peptides in advance. To improve their immuno-stimulatory capacity, AML cells may be modified in vitro to secrete cytokines like GM-CSF (Ho, 2006; Ho, 2007) and IL-2 (Chan, 2006) or express costimulatory molecules like CD80 (Chan, 2006).

For peptide vaccines to be effective, the immunizing peptide must be recognized by the immune system and be able to stimulate anti-tumor immunity. The "ideal target" for an antigen-specific immunotherapy would (1) be expressed by leukemic but not normal cells, (2) be highly expressed by all leukemia cells, including LSCs, (3) be involved in leukemia development, maintenance, and progression, (4) be strongly immunogenic, and (5) when used clinically, be able to stimulate tumor-rejection (Cheever, 2009). Of note, AML vaccines designed to target leukemia-specific antigens - such as mutated nucleophosmin (Greiner, 2005) and leukemia-associated antigens like WT1 (Van Driessche, 2012), proteinase 3 (Rezvani, 2008), and RHAMM/CD168 (Greiner, 2005; Schmitt, 2008) - are currently under development or are being evaluated in clinical trials. 
Regarding APC-based vaccines, AML lysates or peptides can be loaded onto DCs by peptide pulsing, loading, or fusion with AML cells. AML cells can also be differentiated into DCs using cytokines or calcium ionophores to improve their antigen-presenting capabilities (Choudhury, 1999). Leukemic DCs maintain expression of leukemiaassociated antigens, and their therapeutic potential is being evaluated in clinical trials $(\mathrm{Li}$, 2004; Li, 2006; Hicks, 2003). However, if AML cells are converted into immature or IDOexpressing DCs, they can hinder anti-tumor immunity by promoting T-cell tolerance (Curti, 2012). Of course, vaccines can be combined with adjuvants like TLR ligands, cytokines, and co-stimulators to enhance immune activation.

Provenge (Sipuleucel-T), a patient-specific DC-based vaccine for advanced prostate cancer, is the first FDA-approved cellular immunotherapy (Kantoff, 2010). Provenge is prepared by culturing each patient's peripheral blood mononuclear cells with a fusion protein consisting of prostatic acid phosphatase (PAP) linked to granulocytemonocyte colony-stimulating factor (GM-CSF) (Kantoff, 2010). Although the precise mechanism of action is unknown, APCs (including DCs) are believed to ingest, process, and present portions of the GM-CSF protein to T cells to stimulate their anti-tumor activity. Provenge improved survival of prostate cancer patients by 4.1 months (Kantoff, 2010), but this survival benefit has been questioned due to possible flaws in the design of the clinical trial (Huber, 2012). 
Targeting the immuno-suppressive tumor microenvironment

While some therapies are designed to stimulate immune responses, others seek to reduce immuno-suppression. Strategies to inhibit immuno-suppressive cells, factors, and enzymes within the tumor microenvironment include (1) eliminating $T_{\text {regs, }}$ (2) blocking cytokines like TGF- $\beta$, and (3) inhibiting immuno-suppressive enzymes such as indoleamine 2,3-dioxygenase (IDO) (Löb, 2009; Qian, 2009) and arginase (Reisser, 2002). CD25-targeting antibodies eliminate $T_{\text {regs }}$ in the tumor microenvironment and show promise in the treatment of breast cancer (Rech, 2012). Of note, immunecheckpoint receptors can not only inhibit the activity of cytotoxic $T$ cells, they may also enhance the proliferation and activity of Tregs (Pardoll, 2012). Thus, the clinical benefits of immune-checkpoint-receptor inhibitors may be due not only to activation of cytotoxic T cells but also to inhibition of immuno-suppressive Tregs (Pardoll, 2012). Competitive inhibitors or "suicide substrates" have been designed to block the enzymatic activity of IDO (Löb, 2009; Qian, 2009) and arginase (Reisser, 2002) which inhibit T-cell activity by depleting local amino acids. Strategies to neutralize immuno-suppressive factors can act synergistically with immune-stimulating agendas since they affect different mechanisms.

Additional strategies

Immuno-therapeutic strategies reviewed elsewhere include the use of cytokines (i.e. IL-2) (Dranoff, 2004), oncolytic viruses (Chiocca, 2002), targeted anti-cancer therapies that enhance immune responses (Vanneman, 2012), and "conventional" radiotherapies and chemotherapies that induce ICD or modulate the immunogenicity of tumor cells (Kono, 2013; Inoue, 2014). 
Designing and engineering patient-specific immunotherapies for AML

To save the lives of some cancer patients, multi-pronged therapies may be needed that combine patient-specific immune strategies with "conventional" treatments. An immunologic component may be necessary to eliminate the stem-like, relapse-causing cancer cells and to provide long-term protection via "memory" immune-effector cells. The immunogenic death of mature "bulk" cancer cells induced by radiotherapies and some chemotherapies may act synergistically with immunotherapies.

Technically, the successful engineering of patient-specific immune strategies has been possible because better methods have been developed for maintaining and manipulating a patient's tumor and immune cells in vitro. Recently, two small molecules, StemRegenin1 (an aryl-hydrocarbon-receptor antagonist) and UM729 (a pyrimidol indole), were found to inhibit differentiation and maintain AML LSC activity in culture (Pabst, 2014). New approaches and tools are being identified and evaluated on an ongoing basis so patient-specific, anti-CSC immune therapies might, in the future, be developed soon after diagnosis - preferably without genetic engineering. Figure 4.4 provides an overview of diverse tools and conditions that may facilitate in vitro cellengineering. One premise of this project is that any cell-manipulation tasks needed to generate a desired immune therapy - even the de-differentiation of immune and tumor cells - can eventually be accomplished without genetic engineering via the skillful use of molecular tools and physical conditions (Hou, 2013). This optimism is based on the observation that normal cells of primitive animals spontaneously de-differentiate before their amputated limbs regenerate, and malignant human cells can also de-differentiate. That is, even profound cell changes can be induced by physiologic stresses, and these 
molecular mechanisms are likely to be clarified and (eventually) exploited in vitro for therapy-development purposes.

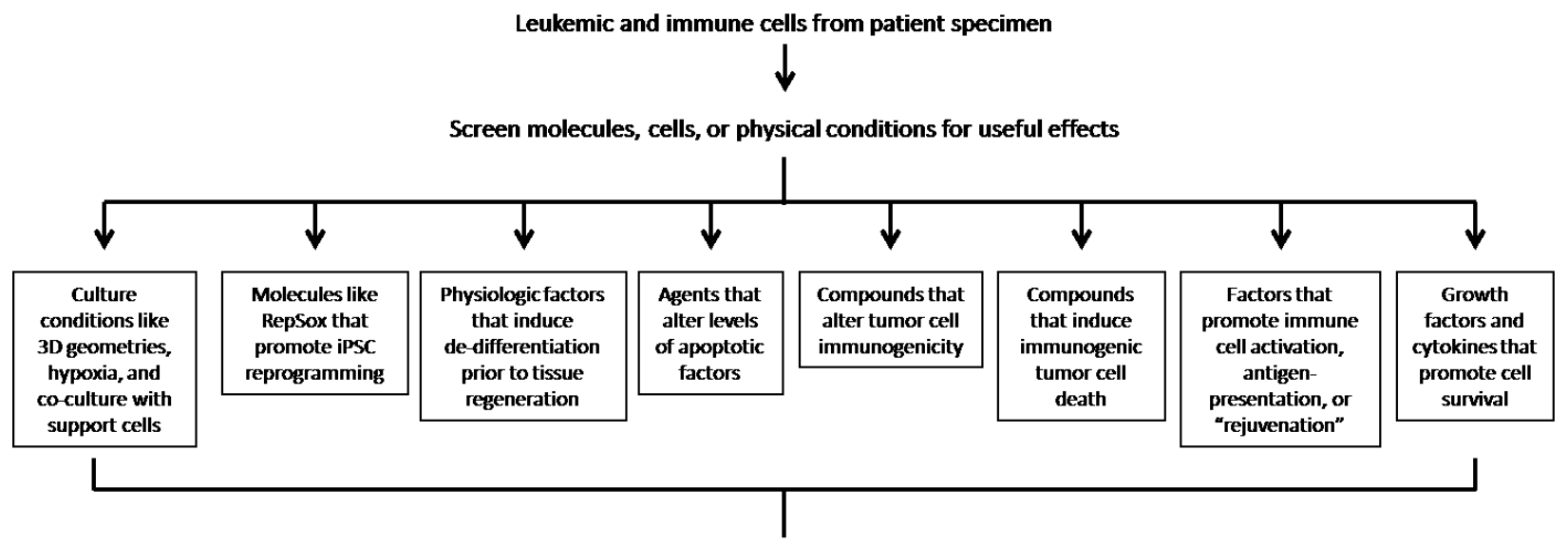

Evaluate effects on tumor cells, immune cells, and tumor cell-immune cell interactions in vitro

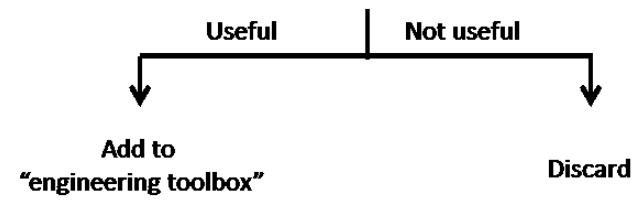

Figure 4.4. Potential "candidate tools" inspired by regeneration and cancer research that may facilitate in vitro engineering of cancer immunotherapies. 
Regarding antibody therapies, to develop and evaluate antibodies for an AML patient's genetically and immuno-phenotypically unique disease, it may be necessary to identify the specific tumor-associated surface antigens on that patient's stem-like AML cells. The engineering and preclinical evaluation of a patient-specific antibody strategy may require that adequate numbers of the patient's primitive leukemia cells be maintained in vitro long enough to complete all the required technical tasks (Pabst, 2014). Currently, finding tools and conditions that promote the survival and expansion of primary leukemia cells remains a priority (Pabst, 2014). Because specimens from some patients contain only small proportions of primitive AML cells, tools may be required to "convert" mature AML cells into stem-like AML cells (Noh, 2012) - see Figure 4.5. When working with the cells available in some patient specimens, de-differentiation may be needed. Fortunately, a variety of cell types can be de-differentiated in vitro using just a few defined factors (Takahashi, 2006; Takahashi, 2007; Hou, 2013). As outlined in Figure 4.4, tools and conditions that may improve survival of stem-like AML cells - or convert mature AML cells into more stem-like cells - include both naturally occurring physiologic factors and artificially synthesized compounds. New candidate tools and conditions for cellengineering are being identified as cell-culture methods improve and new insights are gained into the molecular mechanisms that induce cellular de-differentiation during tissue regeneration and chemical reprogramming. Perhaps, the potent chemicals used for 7-factor reprogramming to pluripotency (Hou, 2013) may be useful for engineering AML immune therapies. Of note, RepSox is one of the seven factors, and, conceivably, the other six chemicals may also help maintain or manipulate primitive AML cells in vitro in useful ways. Presumably, better ways to generate, or maintain, primitive AML cells in 
vitro can help bioengineers design antibodies that target/bind antigens on the stem-like AML cells responsible for disease-relapse.

Of note, because TGF- $\beta$ can increase cell-surface expression of Tim-3 (Wiener, 2006; Kim, 2012) - which is expressed by the highly evolved LSCs found in patients with advanced AML disease - factors like TGF- $\beta$ may be useful when there is a need to evaluate, in vitro, tumor-cell phenotypes in advanced stages of evolution. In general, AML-cell diversity is substantial, and patient-specific strategies may be necessary since each patient's LSC-associated antigens can have unique molecular and cytogenetic features at disease onset as well as after evolution-dependent phenotypes emerge as disease progresses. Since there is an evolving distribution of cancer cells within any given patient, presumably, any factor that can drive AML cells toward any of the CSC phenotypes (Noh, 2012) is potentially useful when engineering antibodies or cells for the purpose of targeting relapse-causing AML cells. 


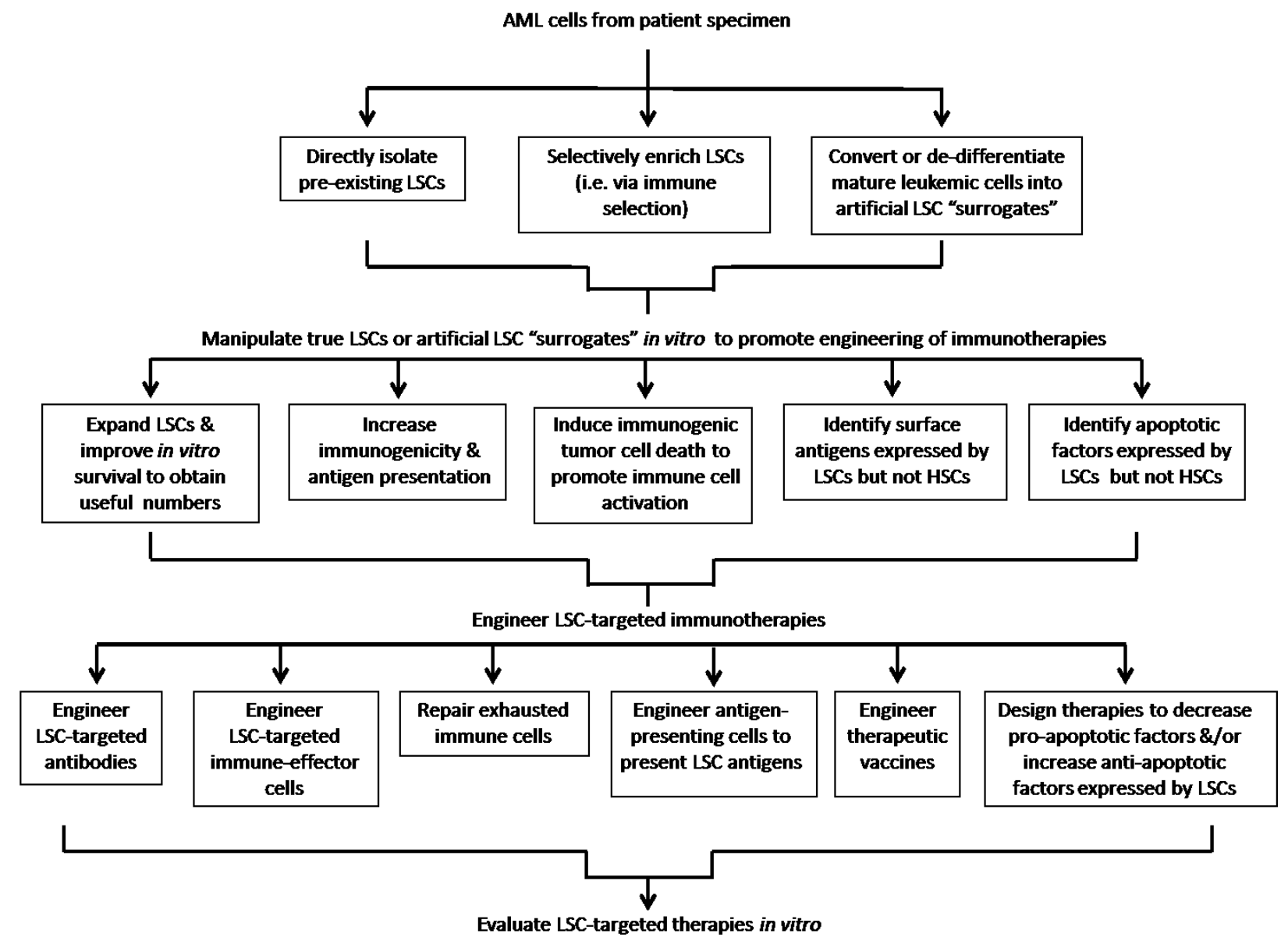

Figure 4.5. Potential ways pre-existing LSCs or in vitro-derived LSC "surrogates" may need to be manipulated for the design of LSC-targeted immunotherapies. Overview of ways to isolate, generate, and/or manipulate a patient's pre-existing LSCs or dedifferentiated LSC "surrogates" prior to engineering and evaluating LSC-targeted therapies.

The engineering of patient-specific immune strategies that involve antigenpresenting and immune-effector cells is likely to be more difficult than engineering antibodies - except, perhaps, in the special (and remarkable) context of the spontaneous activation of $ү \delta \bar{T}$-cells in co-culture systems. In general, immune-cell engineering may involve multiple cells (T cells, NK cells, and APCs as well as tumor cells) manipulated individually and during interactions between these cells (like antigen-presentation and 
immune-synapse formation). Fortunately, ex vivo cell-manipulations provide unlimited options and flexibility. For example, optimal in vitro antigen-presentation and immunecell activation may require using LSC-like cells that are less evolved than those that are used to engineer antibodies since the more evolved LSC phenotypes (that appear in advanced leukemias) may be less immunogenic (Costello, 1999; Costello, 2000; Kikushige; Majeti) and more immuno-suppressive than the less evolved LSCs. That is, different agendas may be facilitated by using cells having specific "task-friendly" phenotypes. Fortunately, factors like TGF- $\beta$ and RepSox can change the phenotypes of cancer cells into phenotypes that may reflect different stages of tumor-cell evolution. In general, cancer researchers are beginning to use in vitro cellular manipulations and observations to invent, develop, and evaluate autologous immune-cell therapies. Advancing these ambitious agendas seems feasible when considering, for example, that even the chemical reprogramming of mature cells to pluripotency has already been achieved (Hou, 2013). Also encouraging, "immune-selection pressures" can progressively "convert" populations of tumor cells into more stem-like populations (Noh, 2012). That is, using methods that have already developed, a spectrum of cancer cells can be generated whose cells exhibit different degrees of "stemness" (Noh, 2012). Similarly, a variety of partially reprogrammed intermediates expressing different degrees of lineage-commitment genes are generated during iPSC reprogramming (Hochedlinger, 2009; Mikkelsen, 2008). Because the underlying "immune-selection" mechanisms that drive cancer cells toward a stem-like phenotype (like the Nanog/Tcl1a/Akt pathway) are being identified (Noh, 2012; Li), it is possible that, when needed, chemical "conversion" of the malignant cells available in specimens into CSCs can be accomplished in vitro 
(Landsberg, 2012). Partial, chemical de-differentiation of cancer cells for therapydevelopment purposes seems feasible given that chemicals are able to reprogram mature, differentiated (albeit normal) cells all the way to pluripotency (Hou, 2013).

Although tumor-reactive immune cells are generated in cancer patients, they often become suppressed and dysfunctional through "exhaustion," anergy, or senescence (Wherry, 2011). T-cell exhaustion is a dysfunctional state in which T cells are unable to attack cancer cells and cannot be activated by tumor antigens (Sakuishi, 2010) - perhaps due to ineffective immunologic synapse formation. Before attempting to stimulate an AML patient's immune system, immune cells that are dysfunctional may need to be repaired so they can respond to immune-stimulating agents. In patients with cancer (Sakuishi, 2010) and chronic viral infections (Blank, 2007; Day, 2006; Jin, 2010), exhausted immune cells are often characterized by expression of immune-checkpoint receptors like Tim-3 (Jin, 2010) and PD-1 (Blank, 2007; Day, 2006; Jin, 2010). Of note, the "exhausted" T-cell clones from patients with cancer (Sakuishi, 2010) and chronic viral infections (Blank, 2007; Day, 2006; Jin, 2010) can, ex vivo, be restored to normal function - and expanded - by first reprogramming them into pluripotent cells and then re-differentiating them into CD8 ${ }^{+}$cytotoxic T cells (Vizcardo, 2013; Nishimura, 2013). In addition to reprogramming, blockade of the immune-checkpoint receptors Tim-3, PD-1, and CTLA-4 (when these receptors are expressed) also enhances anti-tumor immune-cell function (Fourcade, 2010; Hodi, 2010; Sakuishi, 2010).

As cancers progress, Tim-3 on the surfaces of a variety of different cells can increase and play key roles in multiple mechanisms that adversely affect a patient's ability to eliminate tumor cells. Remarkably, Tim-3 contributes to immune-cell dysfunction and 
cancer-cell immune-evasion when expressed by leukemic stem cells (Kikushige, 2010), endothelial cells (Huang, 2010), and cancer-impaired antigen-presenting (Chiba, 2012) and immune-effector (Ndhlovu, 2012) cells. Because Tim-3 impairs anti-tumor immunity when expressed by tumor cells, Tim-3 expression was evaluated on RepSox-exposed AML cells. Also, because reprogramming to pluripotency restores cancer-impaired immune cells (Vizcardo, 2013), it was reasoned that reprogramming tools (like RepSox) might (used alone or in combination with other factors) "convert" immune cells toward stem-like phenotypes and help restore their functionality. In general, "converting" either immune cells or tumor cells into more primitive phenotypes may be needed at different stages of $A M L$ therapy-engineering. In advanced AML disease, expression of Tim-3 on LSCs (Kikushige, 2010) and overexpression of Tim-3 on immune cells (Zhou, 2011) may help explain both the resistance of primitive AML cells to immune attack and the dysfunction of immune cells. However, in view of the diversity of AML cytogenetic and immunophenotypic subtypes, the surface antigens that impair immune responses may vary by patient, and patient-specific approaches may be needed despite the apparently frequent, and consistent, involvement of Tim-3.

The engineering of immunotherapies for AML patients may require not just improving in vitro cell-survival (Pabst, 2014) but a variety of other tasks needed to "invent," produce, and evaluate multi-pronged strategies. For example, studies in humans and animals suggest that the inherent death-resistance of primitive tumor cells contributes to their ability to resist immune-cell attack (Costello, 1999; Costello, 2000) and trigger disease-relapse. Thus, a necessary component of a multi-pronged immune strategy might involve administering a (safe) systemic therapeutic agent that directly reduces the 
death-resistance of stem-like malignant cells. Prior studies suggest that inhibiting antiapoptotic factors (like Mcl-1) - that are especially problematic (and elevated) in primitive, highly evolved malignant cells - can promote immune-mediated clearance of cancer cells and improve survival (Noh, 2012). Chemical inhibitors (Weyhenmeyer, 2012) and cellpenetrating proteins (CPPs) (Wang, 2012) that deliver agents like siRNAs or microRNAs inside death-resistant primitive AML cells may be used to reduce levels of anti-apoptotic factors and sensitize tumor cells to cytotoxic T-cell-mediated apoptosis. As a potential treatment for AML patients, a CPP-siRNA conjugate, for example, that can reduce levels of a problematic anti-apoptotic factor must be safe and selectively target the AML cells.

Although therapy-development options for AML patients are unlimited and setting priorities is challenging, researchers agree that a key goal is finding better ways to maintain and manipulate cancer cells in vitro (Pabst, 2014). Presumably, molecular tools will simplify and expedite in vitro cell engineering so immune therapies might be produced and administered soon after diagnosis. Advances in chemical reprogramming have identified promising candidate tools and methods (Hou, 2013) that seem useful for engineering patient-specific immune therapies. The value of manipulating, activating, and expanding immune cells in vitro has been demonstrated: Engineered immune cells have eliminated relapse-causing CSCs in human and animal trials as well as in pre-clinical studies in vitro.

Chapter $\mathbf{V}$ describes the therapeutic vision that guided this project. This vision emerged after conceptual frameworks for cancer and immunology were integrated with specific research findings and the conclusions drawn from clinical successes. Before designing experiments, it was also considered prudent to envision "worst-case scenarios" 
involving (1) leukemia cells that are highly evolved (immune-evasive and death-resistant), (2) dysfunctional immune cells, and (3) distorted cancer microenvironments that support malignant cells and impair immune responses. Technically, the worst possible patient specimen would contain none (or few) of the critically important cells: no CSCs (target cells), no activated immune-effector cells ( $T$ and NK cells), and no functional antigenpresenting cells. Thus, attention was directed to the potential need to complete multiple, complex in vitro cell-engineering tasks in order to generate patient-specific, multi-pronged immune therapies. This formidable, intimidating possibility, in turn, was juxtaposed with the dramatic cell-engineering advances already achieved by regeneration researchers which, in turn, directed attention to useful small-molecule tools like RepSox. In general, the actions of reprogramming tools suggest they may promote cell "stemness" (and the survival of primitive cells) via their effects on pluripotency genes. Thus, RepSox was screened for its ability to maintain primitive AML cells and/or alter Tim-3 expression (since Tim-3 - when expressed by primitive, highly evolved AML cells that trigger relapse inhibits immune-cell activation and disrupts immune synapses). 


\section{References}

Anderson, A. C. (2012). Tim-3, a negative regulator of anti-tumor immunity. Current Opinion in Immunology, 24(2), 213-216.

Blank, C., \& Mackensen, A. (2007). Contribution of the PD-L1/PD-1 pathway to T-cell exhaustion: an update on implications for chronic infections and tumor evasion. Cancer Immunology, Immunotherapy, 56(5), 739-745.

Bleakley, M., \& Riddell, S. R. (2004). Molecules and mechanisms of the graft-versusleukaemia effect. Nature Reviews Cancer, 4(5), 371-380.

Brentjens, R. J., Davila, M. L., Riviere, I., et al. (2013). CD19-targeted T cells rapidly induce molecular remissions in adults with chemotherapy-refractory acute lymphoblastic leukemia. Science Translational Medicine, 5(177), 177 ra38.

Brentjens, R. J., Rivière, I., Park, J. H., et al. (2011). Safety and persistence of adoptively transferred autologous CD19-targeted T cells in patients with relapsed or chemotherapy refractory B-cell leukemias. Blood, 118(18), 4817-4828.

Burkhardt, U. E., Hainz, U., Stevenson, K., et al. (2013). Autologous CLL cell vaccination early after transplant induces leukemia-specific $\mathrm{T}$ cells. The Journal of Clinical Investigation, 123(9), 3756-3765.

Chan, L., Hardwick, N. R., Guinn, B. A., et al. (2006). An immune edited tumour versus a tumour edited immune system: prospects for immune therapy of acute myeloid leukaemia. Cancer Immunology, Immunotherapy, 55(8), 1017-1024.

Cheever, M.A., Allison, J.P., Ferris, A. S., et al. (2009). The prioritization of cancer antigens: a national cancer institute pilot project for the acceleration of translational research. Clinical Cancer Research, 15(17), 5323-5337.

Chiba, S., Baghdadi, M., Akiba, H., et al. (2012). Tumor-infiltrating DCs suppress nucleic acid-mediated innate immune responses through interactions between the receptor TIM3 and the alarmin HMGB1. Nature Immunology, 13(9), 832-842.

Chiocca, E. A. (2002). Oncolytic viruses. Nature Reviews Cancer, 2(12), 938-950.

Choudhury, A., Liang, J. C., Thomas, E. K., et al. (1999). Dendritic cells derived in vitro from acute myelogenous leukemia cells stimulate autologous, antileukemic T-cell responses. Blood, 93(3), 780-786. 
Costello, R., Mallet, F., Chambost, H., et al. (1999). The immunophenotype of minimally differentiated acute myeloid leukemia (AML-M0): reduced immunogenicity and high frequency of CD34+/CD38-leukemic progenitors. Leukemia, 13(10), 1513-1518.

Costello, R. T., Mallet, F., Gaugler, B., et al. (2000). Human acute myeloid leukemia CD34+/CD38- progenitor cells have decreased sensitivity to chemotherapy and Fasinduced apoptosis, reduced immunogenicity, and impaired dendritic cell transformation capacities. Cancer Research, 60(16), 4403-4411.

Curti, A., Trabanelli, S., Onofri, C., et al. (2010). Indoleamine 2, 3-dioxygenaseexpressing leukemic dendritic cells impair a leukemia-specific immune response by inducing potent T regulatory cells. Haematologica, 95(12), 2022-2030.

Day, C. L., Kaufmann, D. E., Kiepiela, P., et al. (2006). PD-1 expression on HIV-specific T cells is associated with T-cell exhaustion and disease progression. Nature, 443(7109), 350-354.

Dranoff, G. (2004). Cytokines in cancer pathogenesis and cancer therapy. Nature Reviews Cancer, 4(1), 11-22.

Fourcade, J., Sun, Z., Benallaoua, M., et al. (2010). Upregulation of Tim-3 and PD-1 expression is associated with tumor antigen-specific CD8+ $\mathrm{T}$ cell dysfunction in melanoma patients. The Journal of Experimental Medicine, 207(10), 2175-2186.

Greiner, J., Li, L., Ringhoffer, M., et al. (2005). Identification and characterization of epitopes of the receptor for hyaluronic acid-mediated motility (RHAMM/CD168) recognized by CD8+ T cells of HLA-A2-positive patients with acute myeloid leukemia. Blood, 106(3), 938-945.

Greiner, J., Ono, Y., Hofmann, S., et al. (2012). Mutated regions of nucleophosmin 1 elicit both CD4+ and CD8+ T-cell responses in patients with acute myeloid leukemia. Blood, 120(6), 1282-1289.

Gross, G., Waks, T., \& Eshhar, Z. (1989). Expression of immunoglobulin-T-cell receptor chimeric molecules as functional receptors with antibody-type specificity. Proceedings of the National Academy of Sciences, 86(24), 10024-10028.

Hicks, C., Cheung, C., \& Lindeman, R. (2003). Restimulation of tumour-specific immunity in a patient with AML following injection with B7-1 positive autologous blasts. Leukemia Research, 27(11), 1051-1061.

Ho, V. T., Dranoff, G., Pasek, M., et al. (2006). GM-CSF secreting leukemia cell vaccinations after allogeneic non-myeloablative peripheral blood stem cell transplantation 
in patients with advanced myelodysplastic syndrome or refractory acute myeloid leukemia. Biology of Blood and Marrow Transplantation, 12(2), 17-18.

Hochedlinger, K., \& Plath, K. (2009). Epigenetic reprogramming and induced pluripotency. Development, 136(4), 509-523.

Hou, P., Li, Y., Zhang, X., et al. (2013). Pluripotent stem cells induced from mouse somatic cells by small-molecule compounds. Science, 341(6146), 651-654.

Huang, X., Bai, X., Cao, Y., et al. (2010). Lymphoma endothelium preferentially expresses Tim-3 and facilitates the progression of lymphoma by mediating immune evasion. The Journal of Experimental Medicine, 207(3), 505-520.

Huber, M. L., Haynes, L., Parker, C., et al. (2012). Interdisciplinary critique of sipuleucel$\mathrm{T}$ as immunotherapy in castration-resistant prostate cancer. Journal of the National Cancer Institute, 104(4), 273-279.

Inoue, H., \& Tani, K. (2014). Multimodal immunogenic cancer cell death as a consequence of anticancer cytotoxic treatments. Cell Death \& Differentiation, 21(1), 3949 .

Irving, B. A., \& Weiss, A. (1991). The cytoplasmic domain of the T cell receptor $\zeta$ chain is sufficient to couple to receptor-associated signal transduction pathways. Cell, 64(5), 891901.

Jaiswal, S., Jamieson, C. H., Pang, W. et al. (2009). CD47 is upregulated on circulating hematopoietic stem cells and leukemia cells to avoid phagocytosis. Cell, 138(2), 271-285.

Jin, H. T., Anderson, A. C., Tan, W. G., et al. (2010). Cooperation of Tim-3 and PD-1 in CD8 T-cell exhaustion during chronic viral infection. Proceedings of the National Academy of Sciences, 107(33), 14733-14738.

Jin, L., Hope, K. J., Zhai, Q., et al. (2006). Targeting of CD44 eradicates human acute myeloid leukemic stem cells. Nature Medicine, 12(10), 1167-1174.

Jin, L., Lee, E. M., Ramshaw, H. S., et al. (2009). Monoclonal antibody-mediated targeting of CD123, IL-3 receptor a chain, eliminates human acute myeloid leukemic stem cells. Cell Stem Cell, 5(1), 31-42.

Jordan, C. T., Upchurch, D., Szilvassy, S. J., et al. (2000). The interleukin-3 receptor alpha chain is a unique marker for human acute myelogenous leukemia stem cells. Leukemia, 14(10), 1777-1784.

Kalos, M., Levine, B. L., Porter, D. L., et al. (2011). T cells with chimeric antigen receptors have potent antitumor effects and can establish memory in patients with advanced leukemia. Science Translational Medicine, 3(95), 95ra73. 
Kantoff, P. W., Higano, C. S., Shore, N. D., et al. (2010). Sipuleucel-T immunotherapy for castration-resistant prostate cancer. New England Journal of Medicine, 363(5), 411-422.

Kikushige, Y., Shima, T., Takayanagi, S. I., et al. (2010). TIM-3 is a promising target to selectively kill acute myeloid leukemia stem cells. Cell Stem Cell, 7(6), 708-717.

Kim, J. S., Shin, D. C., Woo, M. Y., et al. (2012). T cell immunoglobulin mucin domain (TIM)-3 promoter activity in a human mast cell line. Immune Network, 12(5), 207-212.

Kochenderfer, J. N., Dudley, M. E., Feldman, S. A., et al. (2012). B-cell depletion and remissions of malignancy along with cytokine-associated toxicity in a clinical trial of antiCD19 chimeric-antigen-receptor-transduced T cells. Blood, 119(12), 2709-2720.

Kono, K., Mimura, K., \& Kiessling, R. (2013). Immunogenic tumor cell death induced by chemoradiotherapy: molecular mechanisms and a clinical translation. Cell Death \& Disease, 4(6), e688.

Landsberg, J., Kohlmeyer, J., Renn, M., et al. (2012). Melanomas resist T-cell therapy through inflammation-induced reversible dedifferentiation. Nature, 490(7420), 412-416.

Larson, R. A., Boogaerts, M., Etsey, E. et al. (2002). Antibody-targeted chemotherapy of older patients with acute myeloid leukemia in first relapse using Mylotarg (gemtuzumab ozogamicin). Leukemia, 16(9), 1627-1636.

Li, L., Giannopoulos, K., Reinhardt, P., et al. (2006). Immunotherapy for patients with acute myeloid leukemia using autologous dendritic cells generated from leukemic blasts. International Journal of Oncology, 28(4), 855-861.

Li, Y., \& Laterra, J. (2012). Cancer stem cells: distinct entities or dynamically regulated phenotypes? Cancer Research, 72(3), 576-580.

Li, L., Reinhardt, P., Schmitt, A., et al. (2005). Dendritic cells generated from acute myeloid leukemia (AML) blasts maintain the expression of immunogenic leukemia associated antigens. Cancer Immunology, Immunotherapy, 54(7), 685-693.

Löb, S., Königsrainer, A., Zieker, D., et al. (2009). IDO1 and IDO2 are expressed in human tumors: levo-but not dextro-1-methyl tryptophan inhibits tryptophan catabolism. Cancer Immunology, Immunotherapy, 58(1), 153-157.

Majeti, R., Chao, M. P., Alizadeh, A. A., et al. (2009). CD47 is an adverse prognostic factor and therapeutic antibody target on human acute myeloid leukemia stem cells. Cell, 138(2), 286-299. 
Melero, I., Hervas-Stubbs, S., Glennie, M., Pardoll, D. M., \& Chen, L. (2007). Immunostimulatory monoclonal antibodies for cancer therapy. Nature Reviews Cancer, 7(2), 95-106.

Mikkelsen, T. S., Hanna, J., Zhang, X., et al. (2008). Dissecting direct reprogramming through integrative genomic analysis. Nature, 454(7200), 49-55.

Ndhlovu, L. C., Lopez-Vergès, S., Barbour, J. D., et al. (2012). Tim-3 marks human natural killer cell maturation and suppresses cell-mediated cytotoxicity. Blood, 119(16), 3734-3743.

Nishimura, T., Kaneko, S., Kawana-Tachikawa, A., et al. (2013). Generation of rejuvenated antigen-specific $T$ cells by reprogramming to pluripotency and redifferentiation. Cell Stem Cell, 12(1), 114-126.

Noh, K. H., Kim, B. W., Song, K. H., et al. (2012). Nanog signaling in cancer promotes stem-like phenotype and immune evasion. The Journal of Clinical Investigation, 122(11), 4077-4093.

Obeid, M., Tesniere, A., Ghiringhelli, F., et al. (2007). Calreticulin exposure dictates the immunogenicity of cancer cell death. Nature Medicine, 13(1), 54-61.

Pabst, C., Krosl, J., Fares, I., et al. (2014). Identification of small molecules that support human leukemia stem cell activity ex vivo. Nature Methods, 11(4):436-42.

Pardoll, D. M. (2012). The blockade of immune checkpoints in cancer immunotherapy. Nature Reviews Cancer, 12(4), 252-264.

Porter, D. L., Levine, B. L., Kalos, M., et al. (2011). Chimeric antigen receptor-modified T cells in chronic lymphoid leukemia. New England Journal of Medicine, 365(8), 725-733.

Qian, F., Villella, J., Wallace, P. K., et al. (2009). Efficacy of levo-1-methyl tryptophan and dextro-1-methyl tryptophan in reversing indoleamine-2, 3-dioxygenase-mediated arrest of T-cell proliferation in human epithelial ovarian cancer. Cancer Research, 69(13), 54985504 .

Raval, R. R., Sharabi, A. B., Walker, A. J., et al. (2014). Tumor immunology and cancer immunotherapy: summary of the 2013 SITC primer. Journal for ImmunoTherapy of Cancer, 2(1), 1-11.

Rech, A. J., Mick, R., Martin, S., et al. (2012). CD25 blockade depletes and selectively reprograms regulatory $T$ cells in concert with immunotherapy in cancer patients. Science Translational Medicine, 4(134), 134ra62. 
Reisser, D., Onier-Cherix, N., \& Jeannin, J. F. (2002). Arginase activity is inhibited by LNAME, both in vitro and in vivo. Journal of Enzyme Inhibition and Medicinal Chemistry, 17(4), 267-270.

Rezvani, K., Yong, A. S., Mielke, S., et al. (2008). Leukemia-associated antigen-specific T-cell responses following combined PR1 and WT1 peptide vaccination in patients with myeloid malignancies. Blood, 111(1), 236-242.

Ruggeri, L., Capanni, M., Urbani, E., et al. (2002). Effectiveness of donor natural killer cell alloreactivity in mismatched hematopoietic transplants. Science, 295(5562), 20972100 .

Sakuishi, K., Apetoh, L., Sullivan, J. M., Blazar, B. R., Kuchroo, V. K., \& Anderson, A. C. (2010). Targeting Tim-3 and PD-1 pathways to reverse T cell exhaustion and restore antitumor immunity. The Journal of Experimental Medicine, 207(10), 2187-2194.

Schmitt, M., Schmitt, A., Rojewski, M. T., et al. (2008). RHAMM-R3 peptide vaccination in patients with acute myeloid leukemia, myelodysplastic syndrome, and multiple myeloma elicits immunologic and clinical responses. Blood, 111(3), 1357-1365.

Schlom, J. (2012). Therapeutic cancer vaccines: current status and moving forward. Journal of the National Cancer Institute, 104(8), 599-613.

Takahashi, K., \& Yamanaka, S. (2006). Induction of pluripotent stem cells from mouse embryonic and adult fibroblast cultures by defined factors. Cell, 126(4), 663-676.

Takahashi, K., Tanabe, K., Ohnuki, M., et al. (2007). Induction of pluripotent stem cells from adult human fibroblasts by defined factors. Cell, 131(5), 861-872.

Tivol, E. A., Borriello, F., Schweitzer, A. N., et al. (1995). Loss of CTLA-4 leads to massive lymphoproliferation and fatal multiorgan tissue destruction, revealing a critical negative regulatory role of CTLA-4. Immunity, 3(5), 541-547.

Tesniere, A., Panaretakis, T., Kepp, O., et al. (2008). Molecular characteristics of immunogenic cancer cell death. Cell Death \& Differentiation, 15(1), 3-12.

Themeli, M., Kloss, C. C., Ciriello, G., et al. (2013). Generation of tumor-targeted human $\mathrm{T}$ lymphocytes from induced pluripotent stem cells for cancer therapy. Nature Biotechnology, 31(10), 928-933.

Tran, E., Turcotte, S., Gros, A., et al. (2014). Cancer immunotherapy based on mutationspecific CD4+ T Cells in a patient with epithelial cancer. Science, 344(6184), 641-645. 
Van Driessche, A., Berneman, Z. N., \& Van Tendeloo, V. F. (2012). Active specific immunotherapy targeting the Wilms' tumor protein 1 (WT1) for patients with hematological malignancies and solid tumors: lessons from early clinical trials. The Oncologist, 17(2), 250-259.

Vanneman, M., \& Dranoff, G. (2012). Combining immunotherapy and targeted therapies in cancer treatment. Nature Reviews Cancer, 12(4), 237-251.

Vizcardo, R., Masuda, K., Yamada, D., et al. (2013). Regeneration of human tumor antigen-specific T cells from iPSCs derived from mature CD8+ T Cells. Cell Stem Cell, 12(1), 31-36.

Wang, H. Y., \& Wang, R. F. (2012). Enhancing cancer immunotherapy by intracellular delivery of cell-penetrating peptides and stimulation of pattern-recognition receptor signaling. Advances in Immunology, 114, 151-176.

Waterhouse, P., Penninger, J. M., Timms, E., et al. (1995). Lymphoproliferative disorders with early lethality in mice deficient in Ctla-4. Science, 270(5238), 985-988.

Weyhenmeyer, B., Murphy, A. C., Prehn, J. H., et al. (2012). Targeting the anti-apoptotic Bcl-2 family members for the treatment of cancer. Experimental Oncology, 34(3), 192199.

Wherry, E. J. (2011). T cell exhaustion. Nature Immunology, 12(6), 492-499.

Wiener, Z., Kohalmi, B., Pocza, P., et al. (2006). TIM-3 is expressed in melanoma cells and is upregulated in TGF- $\beta$ stimulated mast cells. Journal of Investigative Dermatology, 127(4), 906-914.

Yao, S., Zhu, Y., \& Chen, L. (2013). Advances in targeting cell surface signalling molecules for immune modulation. Nature Reviews Drug Discovery, 12(2), 130-146.

Zhang, Y., Ma, C. J., Wang, J. M., et al. (2012). Tim-3 regulates pro- and antiinflammatory cytokine expression in human CD14+ monocytes. Journal of Leukocyte Biology, 91(2), 189-196.

Zhou, Q., Munger, M. E., Veenstra, R. G., et al. (2011). Coexpression of Tim-3 and PD-1 identifies a CD8+ T-cell exhaustion phenotype in mice with disseminated acute myelogenous leukemia. Blood, 117(17), 4501-4510.

Zitvogel, L., Apetoh, L., Ghiringhelli, F., \& Kroemer, G. (2008). Immunological aspects of cancer chemotherapy. Nature Reviews Immunology, 8(1), 59-73. 
Zitvogel, L., Tesniere, A., \& Kroemer, G. (2006). Cancer despite immunosurveillance: immunoselection and immunosubversion. Nature Reviews Immunology, 6(10), 715-727. 


\section{Chapter V}

Guiding therapeutic vision 
An intentionally broad, and unassuming, therapeutic vision was used to set experimental priorities. This vision was inspired by advances in cancer immunotherapy and stem-cell technology (noted in Figure 5.1). Described here are key research and clinical findings and the underlying rationale for a multi-pronged, immune-based strategy.

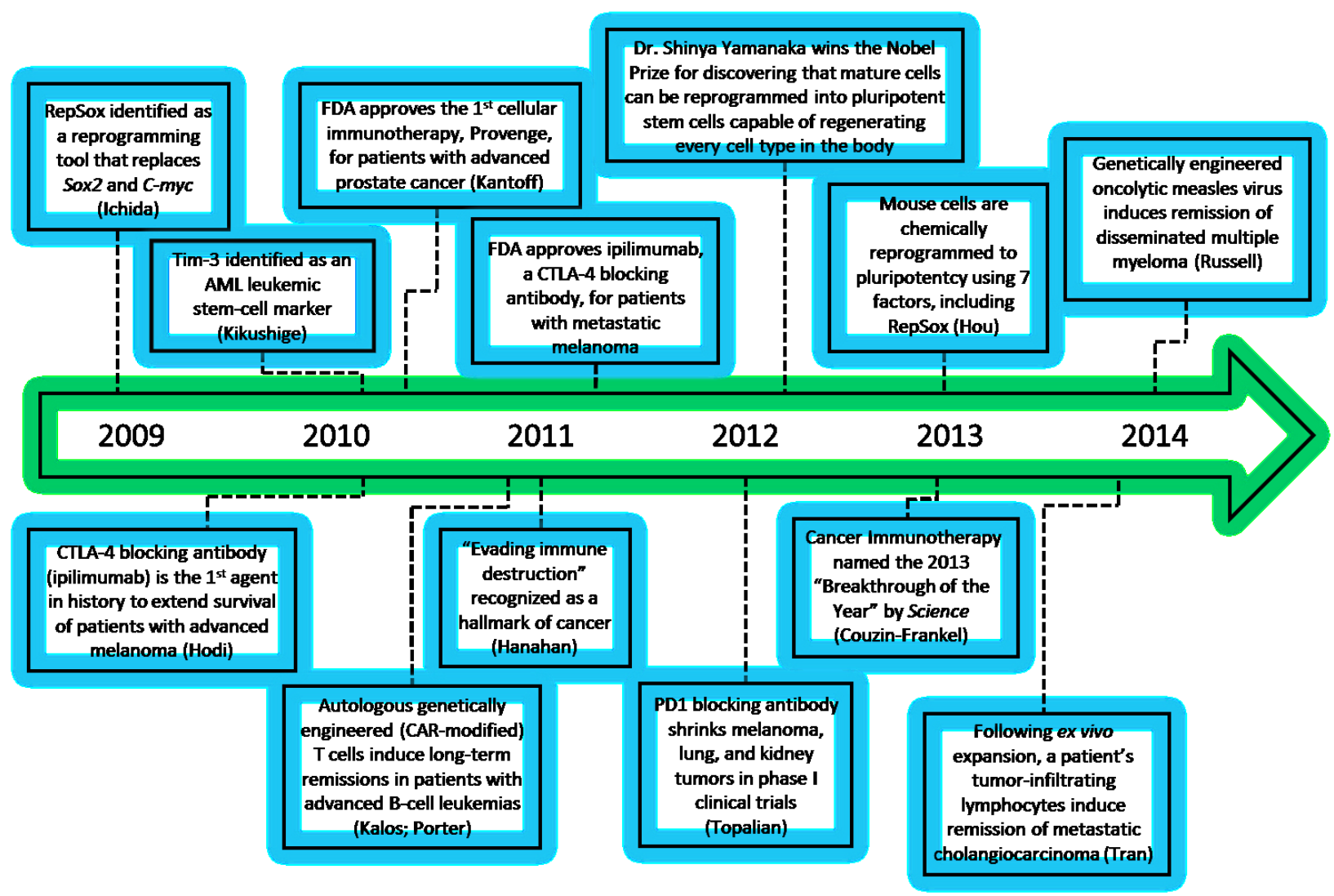

Figure 5.1. Timeline of significant events during thesis. 


\section{Key features of an ideal anti-cancer immunotherapeutic strategy and underlying rationale}

Utilize a multi-pronged approach

In order to cure leukemia, therapies may require overcoming multiple interrelated mechanisms of immune resistance by (1) increasing the poor immunogenicity of leukemia cells, (2) activating and repairing dysfunctional antigen-presenting and immune-effector cells, and (3) inhibiting immuno-suppressive elements of tumor microenvironments (outlined in Figure 4.1). Of note, perhaps some of these tasks are best visualized as cellmanipulations designed to improve the activation of immune cells in vitro by enhancing the immunologic synapse between leukemia and immune cells. A variety of therapeutic interventions involving activated immune cells, antibodies, and agents that increase tumor-cell immunogenicity and/or induce immunogenic tumor-cell death may be needed to generate an effective anti-tumor immune response. Therapies may unleash/reactivate pre-existing immune responses (i.e. tumor-reactive lymphocytes that have become suppressed) or induce new anti-tumor immune reactions (i.e. by releasing previously unexposed tumor antigens or by presenting antigens already being displayed in a new, pro-inflammatory context). The ultimate goal is to eradicate disease-sustaining LSCs and induce immunologic memory that protects against relapse. In clinical trials, skillfully designed immune therapies have eliminated quiescent, stem-like tumor cells that sustain disease and trigger relapse, and "memory" immune cells have been detected that should provide long-term protection by detecting, and responding to, the re-emergence of CSCs. 


\section{Personalize therapy for each cancer patient}

Patient-specific elements: Technical breakthroughs are suggesting new and diverse ways to repair a cancer patient's dysfunctional immune cells (Hodi, 2010; Kalos, 2011; Porter, 2011; Reiners, 2013). Remarkably, the anti-tumor function of cancerimpaired immune cells can be restored so these cells can be used therapeutically (see Figure 5.2). Defects in antigen-presentation by DCs can be overcome by ex vivo loading with tumor peptides (Kantoff, 2010). Cytotoxic killing and/or cytokine release by NK and T cells can be rescued by immune-checkpoint blockade (Hodi, 2010), reprogramming to pluripotency (Vizcardo, 2013; Themeli, 2013), and the physical targeting of immune cells against cancer cells using bispecific antibodies (Reiners, 2013). Fortunately, using the patient's own immune cells as therapy eliminates the risk of host rejection (since autologous immune cells are not "foreign") and graft-versus-host disease (GvHD). Especially encouraging, patient-specific anti-cancer immune therapies involving chimeric antigen receptor (CAR)-modified T cells have induced long-term remissions in patients with advanced leukemia who failed all prior therapies (Kalos, 2011; Porter, 2011). In addition, patient-specific DC vaccines for patients with advanced prostate cancer are now FDA-approved (Kantoff, 2010). 

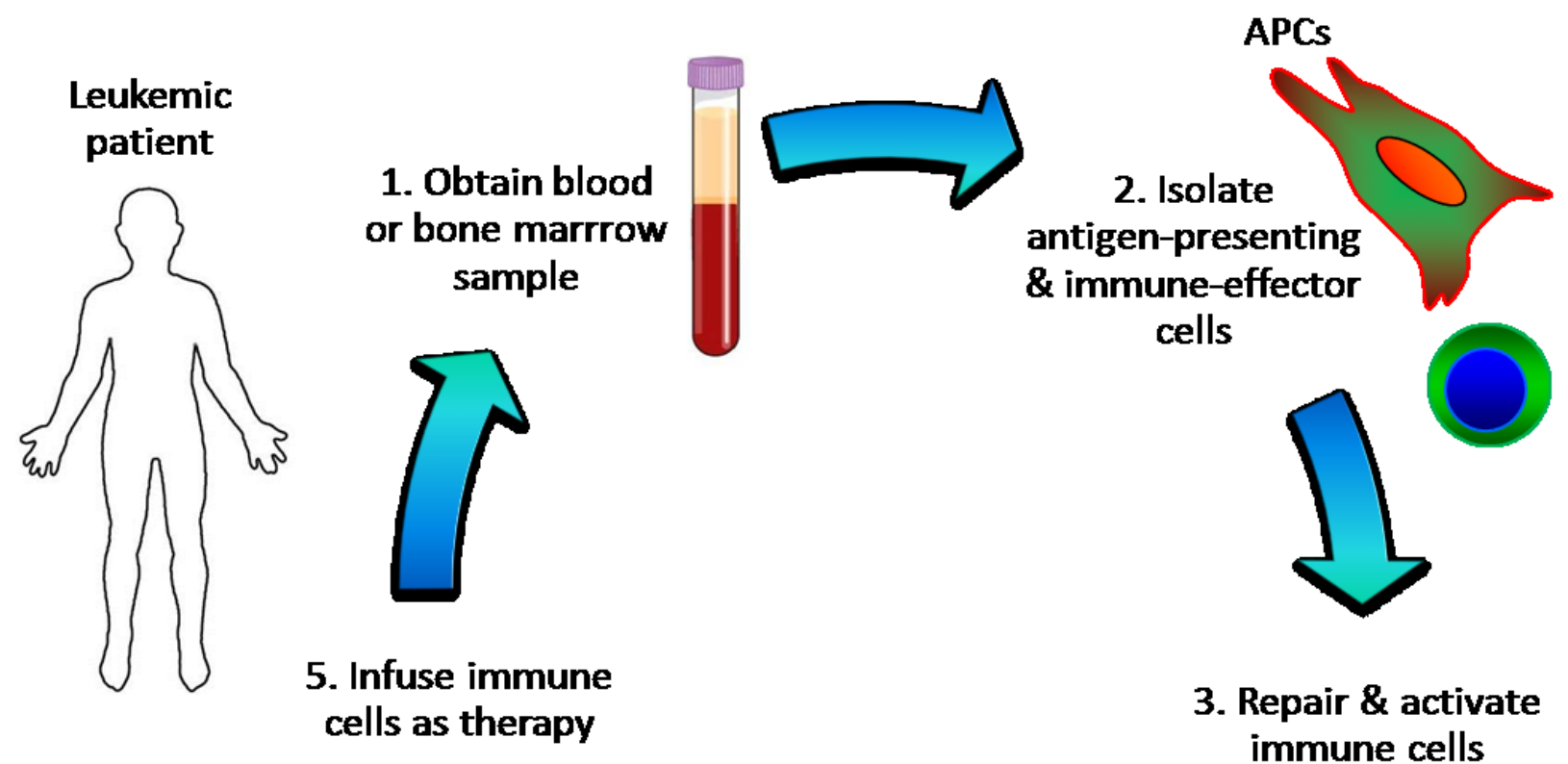

5. Infuse immune cells as therapy

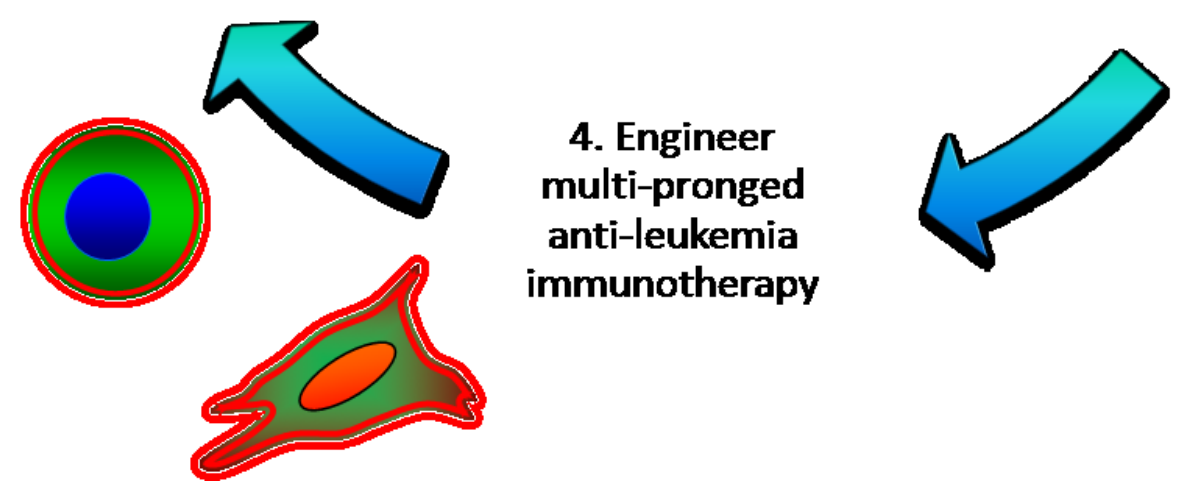

3. Repair $\&$ activate immune cells

Figure 5.2. Patient-specific cellular immunotherapy. Based on patient-to-patient differences in targetable AML antigens and immune cell dysfunctions, some components of an immune therapy may need to be patient-specific. Following isolation of an AML patient's immune cells (some or all of which may be dysfunctional), they can be repaired, activated, and/or engineered against each patient's genetically unique tumor prior to use as therapy. 
Personalized elements: Each patient's disease is genetically unique and has evolved in different settings. Tumor-cell surface-antigens, molecular defects, and mechanisms of immune resistance vary by patient as well as time of diagnosis. For example, cancer-related features can vary in terms of the specific immune-cell defects (i.e. anergy, exhaustion; expression of Tim-3, PD-1, or CTLA-4), immuno-suppressive cell types (i.e. Tregs, MDSCs, TAMs), and the presence, or functionality, of tumor-reactive immune cells. Ideally, choosing pre-existing - and/or engineering new - therapeutic antibodies, immunogenicity-altering compounds, inhibitors of anti-apoptotic factors, and immuno-modulatory agents (for in vivo or in vitro use) should be based on patient-specific technical insights.

In vitro cell-engineering using molecular tools

In some cases, leukapheresis specimens will contain all the cells needed to engineer and evaluate a patient-specific immunotherapy: the LSCs to be targeted, normal HSCs to be spared, and the immune-effector cells and/or APCs to be engineered before being administered therapeutically. Multiple tools (see Figure 4.4) may be needed to alter cancer cells (see Figure 4.5) and repair dysfunctional antigen-presenting and immune-effector cells. Fortunately, immune-effector cells can be activated ex vivo, away from immuno-suppressive tumor microenvironments. Small molecules and chemical engineering methods may simplify and expedite the development of patient-specific immunotherapies and, perhaps, completely eliminate the need for genetic engineering.

As noted, to develop patient-specific immunotherapies, engineering both tumor and immune cells may be necessary. Tumor cells may need to be altered to increase immunogenicity and/or maintain or induce "stemness" while immune cells may need to 
be repaired and activated. Altering cells by exposure to chemical compounds is simpler and faster than genetic engineering and avoids the fears and safety concerns associated with genetically modified cells. In vitro, primary cells can be exposed to environmental conditions and chemical factors that directly alter cells or that select for (via "survival of the fittest") a subset of cells of therapeutic interest. Although tumor and normal cells are different, tools used for tissue regeneration and the de-differentiation of normal cells might also predictably affect cancer cells. For example, tools that induce Nanog not only help reprogram normal cells into stem cells, they can also induce a "stem-like" phenotype in some cancer cells (Ichida, 2009; Noh, 2012). Furthermore, the effects of chemical compounds are often reversible, allowing greater flexibility and control over the order/sequence and duration of cellular alterations. To illustrate how cell-engineering tasks can vary by the therapeutic purpose, in order to enhance immune-cell activation in vitro, it may be best to increase the immunogenicity of CSCs by changing their phenotype to a less-evolved CSC phenotype. In contrast, when developing antibody therapies, in order to know what new antigens might be expressed by highly evolved CSCs, immuneselection or therapeutic pressures could be used to drive CSCs to more highly evolved CSC phenotypes. Considering the diversity of the cell contents in specimens from different leukemia patients, when only mature leukemia cells are present in a patient's specimen, de-differentiation of the available leukemia cells into LSC-like surrogates may be necessary in order to develop therapies that can target the relapse-causing LSCs.

Administering immunotherapies as soon as possible after diagnosis

Ideally, immune therapies should be administered as soon as possible after initial diagnosis since, via "immunoediting," CSCs can evolve into more problematic CSCs that, 
for example, might express Tim-3. That is, as cancers become more advanced, tumor cells become more immune- and death-resistant as the immune system selects for "immune-escape variants" (Dunn, 2002; Noh, 2012). Over time, immune cells become progressively more impaired and exhausted the longer they are exposed to immunosuppressive factors within tumor microenvironments such as soluble factors (like IL-10, and TGF- $\beta$ ), enzymes (like arginase, IDO), immuno-suppressive cells (like $\mathrm{T}_{\text {regs, }}$ MDSCs, TAMs), and immune-checkpoint receptors (like Tim-3, PD-1, CTLA-4). As malignant diseases progress, tumor microenvironments also become progressively more distorted as tumor cells disrupt local oxygen and nutrient levels and convert stromal cells into CAFs.

\section{Encouraging research findings relevant to the therapeutic vision}

Breakthroughs in the reprogramming of mature (normal) cells to pluripotency which resembles the de-differentiation that occurs during tissue regeneration in primitive animals - have identified molecular tools that seem useful for replicating complex in vivo processes (Feng, 2009; Ichida, 2009; Hou, 2013). Although the stimuli and cues that trigger or coordinate the cellular de-differentiation that precedes tissue regeneration are difficult to dissect and clarify in vivo, advances in iPSC reprogramming have identified potent molecular compounds through unbiased chemical screens (Ichida, 2009). RepSox, an inhibitor of TGF- $\beta$ receptor 1 , is a potent chemical reprogramming tool that replaces Sox2 and $M Y C$ - two of the four reprogramming factors that previously required viral delivery (Ichida, 2009). Later, a combination of seven small molecules was found to reprogram normal mouse cells to pluripotency without genetic engineering (Hou, 2013). Remarkably, in vitro reprogramming of cancer-exhausted $\mathrm{T}$ cells to pluripotency followed by re-differentiation into $\mathrm{T}$ cells - was found to restore their anti-tumor activity 
and increase their expansion potential, while retaining their antigen specificity (Vizcardo, 2013). These technical breakthroughs in cell reprogramming suggest that the cell transformations needed to generate anti-cancer therapies might (eventually) also be induced in vitro using chemical compounds (Hou, 2013).

Animal studies have confirmed how combinations of strategies can cooperate to more effectively eliminate cancer cells and prolong survival. In mice with colon cancer, survival was dramatically extended when an agent that inhibits the generation of an antiapoptotic factor was administered along with the antigen-specific T-cell therapy (Noh, 2012). Combining the co-blockade of multiple immune-checkpoint receptors with the activation of T-cell co-stimulatory receptors has also improved outcomes (Fourcade, 2010). Combining targeted anti-cancer agents with systemic agents that induce immunogenic cell death, such as radiotherapies and some chemotherapies (like anthracyclines), may also be useful. In addition, the CSC theory (discussed in Chapter I) and studies of MRD cells suggest CSCs must be targeted because they trigger relapses as well as initiate disease (Gerber, 2012). Thus, for AML patients, maintaining - or generating - their critical LSC-like cells in vitro can be a vital technical priority.

\section{Role and implications of the therapeutic vision for this project}

Encouraged by the cell-engineering successes of regeneration researchers, RepSox was screened for its ability to maintain and manipulate the primitive CD34 ${ }^{+} \mathrm{AML}$ cells that often contain the LSCs thought to trigger AML relapse. Of course, enthusiasm for this project increased when patient-specific immune therapies were found to save the lives of cancer patients (Kalos, 2011; Porter, 2011; Tran, 2014) and when new chemical tools and in vitro methods were developed that could manipulate primary cells in ways 
relevant for therapy-development (Hou, 2013; Pabst, 2014).

To plan experiments - and the collection and processing of AML specimens patient-specific anti-AML therapies considered promising were envisioned. Thinking "backwards" from an aggressive anti-LSC immune strategy was useful because attention was directed to technical obstacles and priorities. One basic challenge seemed to be the need to better maintain and manipulate a patient's AML cells in vitro. When considering the engineering of immune therapies, attention is immediately directed to a variety of technical tasks: ways to generate and evaluate therapies involving antibodies, activated immune-effector cells, antigen-presenting cells, and/or agents that can improve the immunogenicity or apoptotic susceptibility of tumor cells. To better activate immune cells, tools may be needed to render tumor cells more immunogenic - perhaps by inducing a less evolved CSC phenotype. In contrast, when investigating antigens on highly evolved CSCs, immune-selection and therapeutic pressures might be used to "convert" CSCs into more highly evolved CSCs that are less immunogenic due to the expression of antigens like Tim-3.

This study was influenced by the CSC theory, new immunologic concepts, recent research findings, and recent patient-specific immunotherapies engineered for leukemia (Kalos, 2011; Porter, 2011) and prostate cancer (Kantoff, 2010). Because patient-specific treatments may be needed to save the lives of some AML patients, AML cells from leukapheresis specimens were used instead of leukemic cell-lines despite the extra time and challenges associated with collecting, processing, and maintaining primary cells. Also, working with cells from diverse AML patients was considered more important than using a cell-line to expedite and simplify the experimental agenda. Thus, the basic 
challenge of maintaining and manipulating a patient's primitive cells had to be confronted as well as the challenges highlighted by the CSC theory. Although an AML patient's leukemic stem cells (LSCs) represent the critical target conceptually, in the context of constant tumor-cell "immunoediting," the identity of the most recently evolved CSCs is impossible to know with any confidence. Thus, the frustrating paradox: a patient's CSCs are of utmost importance - but, their most recent identity and locations are unknown. Also, the CSCs capable of inducing cancer relapses may be a distribution of diverse location-specific CSCs with phenotypes ranging from "minimally evolved" to "highly evolved." That is, CSCs may vary by location as they interact locally with different cancerdistorted immune cells and microenvironments. Immune-selection pressures in vivo "convert" CSCs into progressively more therapy-resistant and less immunogenic cells. This steady "conversion" of cancer cells (into cells that can engraft cancer with progressively greater efficiency) has been demonstrated in vitro as well as in animals (Noh, 2012). Thus, it seems prudent to assume it is impossible to know what are the most recently evolved (and most lethal) CSCs sequestered in protective niches and, therefore, the sooner immune therapies are administered, the better. However, although CSCs are constantly evolving in vivo, human and animal studies suggest immune therapies engineered in vitro can successfully eliminate relapse-causing cells (Kalos, 2011; Porter, 2011). That is, some leukemia patients have still not relapsed, and this success is consistent with evidence of long-term immunologic memory (Kalos, 2011; Porter, 2011). In one animal study, the observation and manipulation of immune and tumor cells in vitro inspired a 2-pronged immune strategy that was effective in vivo even though the tumor cells used to design and evaluate the immunotherapy were not 
"conventional CSCs," but stem-like surrogates (Noh, 2012). That is, to design, create, and preclinically test immunotherapies in vitro, it may not be necessary that the stem-like tumor cells used for therapy-development be identical to the most recently evolved CSCs in vivo. Overall, it seems prudent to assume that immune strategies engineered and administered soon after the onset of cancer are more likely to be effective by minimizing the duration of CSC evolution. It seems the CSC and immunoediting theories can provide practical guidance even though, paradoxically, the phenotype of a patient's most recently evolved CSCs may never be known with certainty.

By combining such considerations with what is known about AML disease, a multipronged therapeutic strategy was envisioned before specific experiments were planned. This vision identified a need for tools and methods that could better maintain primitive AML cells in vitro. Thus, RepSox and other factors were assessed for their ability to prolong survival of primitive AML CD $34^{+}$cells. As noted, RepSox had attracted attention because of its known actions on normal cells and its role as a potent reprogramming tool (Ichida, 2009; Hou, 2013). In retrospect, considering the actions of RepSox identified in prior studies, it is not surprising RepSox can slow the loss of primitive AML cells in vitro.

Expression of Tim-3 on AML cells was measured because the therapeutic vision suggested it is important to (1) understand, identify, and process stem-like AML cells [Tim-3 is considered a marker of (highly evolved) LSCs (Jan, 2011)] and (2) determine if Tim-3 can be reduced since Tim-3 may be responsible for tumor-cell immune evasion and distortion of immunologic synapses between LSCs and immune cells. If Tim-3 expression by tumor cells can be reduced, immune-cell activation in vitro might be enhanced.

Thus, the therapeutic vision and conceptual frameworks directed attention to 
specific experiments. Chapter VI describes the effects of RepSox on primary AML cells. When viewed in the context of (1) the relationship between TGF- $\beta$ and Tim-3 (Gellibert, 2004) and (2) the de-differentiation of mature cells to pluripotency (Ichida, 2009), RepSox was considered potentially useful for confronting two key obstacles: LSCs rapidly die or differentiate in culture (making them difficult to study and manipulate) and highly evolved LSCs that express Tim-3 are poorly immunogenic. In the study described in Chapter VI, RepSox is found to slow decay of $\mathrm{CD}_{3} 4^{+} \mathrm{AML}$ cells and accelerate loss of Tim-3, an immune-checkpoint receptor that impairs anti-tumor immunity and disrupts immunologic synapses. Thus, used as an in vitro cell-engineering tool, RepSox may facilitate the production of patient-specific immune therapies designed to target AML LSCs. 


\section{References}

Couzin-Frankel, J. (2013). Cancer immunotherapy. Science, 342(6165), 1432-1433.

Dunn, G. P., Bruce, A. T., Ikeda, H., et al. (2002). Cancer immunoediting: from immunosurveillance to tumor escape. Nature Immunology, 3(11), 991-998.

Feng, B., Ng, J. H., Heng, J. et al. (2009). Molecules that promote or enhance reprogramming of somatic cells to induced pluripotent stem cells. Cell Stem Cell, 4(4), 301-312.

Fourcade, J., Sun, Z., Benallaoua, M., et al. (2010). Upregulation of Tim-3 and PD-1 expression is associated with tumor antigen-specific CD8+ $\mathrm{T}$ cell dysfunction in melanoma patients. The Journal of Experimental Medicine, 207(10), 2175-2186.

Gellibert, F., Woolven, J., Fouchet, M. H., et al. (2004). Identification of 1, 5-naphthyridine derivatives as a novel series of potent and selective TGF- $\beta$ type I receptor inhibitors. Journal of Medicinal Chemistry, 47(18), 4494-4506.

Gerber, J. M., Smith, B. D., Ngwang, B., et al. (2012). A clinically relevant population of leukemic CD34+ CD38- cells in acute myeloid leukemia. Blood, 119(15), 3571-3577.

Hanahan, D., \& Weinberg, R. A. (2011). Hallmarks of cancer: the next generation. Cell, 144(5), 646-674.

Hodi, F. S., O'Day, S. J., McDermott, D. F., et al. (2010). Improved survival with ipilimumab in patients with metastatic melanoma. New England Journal of Medicine, 363(8), 711-723.

Hou, P., Li, Y., Zhang, X., et al. (2013). Pluripotent stem cells induced from mouse somatic cells by small-molecule compounds. Science, 341(6146), 651-654.

Ichida, J. K., Blanchard, J., Lam, K., et al. (2009). A small-molecule inhibitor of TGF- $\beta$ signaling replaces sox2 in reprogramming by inducing nanog. Cell Stem Cell, 5(5), 491503.

Jan, M., Chao, M. P., Cha, A. C., et al. (2011). Prospective separation of normal and leukemic stem cells based on differential expression of TIM3, a human acute myeloid leukemia stem cell marker. Proceedings of the National Academy of Sciences, 108(12), 5009-5014.

Kalos, M., Levine, B. L., Porter, D. L., et al. (2011). T cells with chimeric antigen receptors have potent antitumor effects and can establish memory in patients with advanced leukemia. Science Translational Medicine, 3(95), 95ra73-95ra73. 
Kantoff, P. W., Higano, C. S., Shore, N. D., et al. (2010). Sipuleucel-T immunotherapy for castration-resistant prostate cancer. New England Journal of Medicine, 363(5), 411-422.

Kenter, G. G., Welters, M. J., Valentijn, A. R. P., et al. (2009). Vaccination against HPV16 oncoproteins for vulvar intraepithelial neoplasia. New England Journal of Medicine, 361(19), 1838-1847.

Kikushige, Y., Shima, T., Takayanagi, S. I., et al. (2010). TIM-3 is a promising target to selectively kill acute myeloid leukemia stem cells. Cell Stem Cell, 7(6), 708-717.

Noh, K. H., Kim, B. W., Song, K. H., et al. (2012). Nanog signaling in cancer promotes stem-like phenotype and immune evasion. The Journal of Clinical Investigation, 122(11), 4077.

Pabst, C., Krosl, J., Fares, I., et al. (2014). Identification of small molecules that support human leukemia stem cell activity ex vivo. Nature Methods, 11(4):436-42.

Porter, D. L., Levine, B. L., Kalos, M., et al. (2011). Chimeric antigen receptor-modified T cells in chronic lymphoid leukemia. New England Journal of Medicine, 365(8), 725-733.

Reiners, K. S., Kessler, J., Sauer, M., et al. (2013). Rescue of impaired NK cell activity in hodgkin lymphoma with bispecific antibodies in vitro and in patients. Molecular Therapy, 21(4), 895-903.

Russell, S. J., Federspiel, M. J., Peng, K. W., et al. (2014). Remission of disseminated cancer after systemic oncolytic virotherapy. Mayo Clinic Proceedings, 89(7), 926-933.

Themeli, M., Kloss, C. C., Ciriello, G., et al. (2013). Generation of tumor-targeted human $\mathrm{T}$ lymphocytes from induced pluripotent stem cells for cancer therapy. Nature Biotechnology, 31(10), 928-933.

Topalian, S. L., Hodi, F. S., Brahmer, J. R., et al. (2012). Safety, activity, and immune correlates of anti-PD-1 antibody in cancer. New England Journal of Medicine, 366(26), 2443-2454.

Tran, E., Turcotte, S., Gros, A., et al. (2014). Cancer immunotherapy based on mutationspecific CD4+ T cells in a patient with epithelial cancer. Science, 344(6184), 641-645.

Vizcardo, R., Masuda, K., Yamada, D., et al. (2013). Regeneration of human tumor antigen-specific T cells from iPSCs derived from mature CD8+ T cells. Cell Stem Cell, 12(1), 31-36. 
Zhou, Q., Munger, M. E., Veenstra, R. G., et al. (2011). Coexpression of Tim-3 and PD-1 identifies a CD8+ T-cell exhaustion phenotype in mice with disseminated acute myelogenous leukemia. Blood, 117(17), 4501-4510. 


\section{Chapter VI}

RepSox Slows Decay of CD34 ${ }^{+}$Acute Myeloid Leukemia Cells and Decreases T Cell Immunoglobulin Mucin-3 (Tim-3) Expression

Audrey N. Jajosky, James E. Coad, Jeffrey A. Vos, Karen H. Martin, Jamie R. Senft, Sharon L. Wenger, and Laura F. Gibson 


\section{Abstract}

Despite initial response to therapy, most acute myeloid leukemia (AML) patients relapse. To eliminate relapse-causing leukemic stem/progenitor cells (LPCs), patient-specific immune therapies may be required. In vitro cellular engineering may require (1) increasing the "stemness" or immunogenicity of tumor cells and (2) activating or restoring cancer-impaired immune-effector and antigen-presenting cells. Leukapheresis samples provide the cells needed to engineer therapies: LPCs to be targeted, normal hematopoietic stem cells to be spared, and cancer-impaired immune cells to be repaired and activated. This study sought to advance development of LPC-targeted therapies by exploring non-genetic ways to slow the decay and increase the immunogenicity of primary $\mathrm{CD}^{+} 4^{+} \mathrm{AML}$ cells. $\mathrm{CD} 34^{+} \mathrm{AML}$ cells generally displayed greater colony-forming and aldehyde dehydrogenase (ALDH) activity than CD34- AML cells. Along with exposure to bone marrow stromal cells and low (1-5\%) oxygen, culture with RepSox (a reprogramming tool and TGF- $\beta R 1$ inhibitor) consistently slowed decline of CD34+ AML and myelodysplastic syndrome (MDS) cells. RepSox-treated AML cells displayed higher CD34, CXCL12, and MYC mRNA levels than DMSO-treated controls. RepSox also accelerated loss of T cell immunoglobulin mucin-3 (Tim-3), a receptor that impairs antitumor immunity, from the surface of AML/MDS cells. Our results suggest RepSox may reduce Tim-3 expression by inhibiting TGF- $\beta$ signaling and slow decay of CD34+ AML cells by increasing CXCL12 and MYC, two factors that inhibit AML cell differentiation. By prolonging survival of $\mathrm{CD} 34^{+} \mathrm{AML}$ cells and reducing Tim-3, RepSox may promote in vitro immune cell activation and advance development of LPC-targeted therapies. 


\section{Introduction}

Leukemic stem/progenitor cells (LPCs) are believed to sustain disease, persist after chemotherapy and radiation, and contribute to post-treatment relapses. In any given acute myeloid leukemia (AML) patient, these disease-causing cells may encompass a distribution of diverse immunophenotypes that evolves as disease progresses. Over time, leukemia disrupts the bone marrow (BM), suppresses the immune system, and evolves new "immune-escape" and "growth-advantaged" variants [1, 2]. AML LPCs are often distinctive at the molecular level because of high levels of $T$ cell immunoglobulin mucin-3 (Tim-3) [3, 4] and anti-apoptotic factors [5] as well as low immunogenicity [6]. In the setting of minimal residual disease (MRD), immunotherapy may be needed to eliminate quiescent LPCs spared by conventional therapies. Prompt treatment [7] with antibodies, inhibitors of anti-apoptotic factors, and/or immune cells that target LPCs while sparing normal hematopoietic stem cells (HSCs) - should improve AML patient survival, especially when combined with immune-modulating strategies and chemotherapies that cause immunogenic tumor cell death.

Regarding development of patient-specific LPC-targeted therapies, recent technical advances are encouraging. Notably, immune cell activity - impaired by leukemic disease states - can be restored in patients after their immune cells have been manipulated in vitro $[8,9]$. When treated with their own custom-engineered $\mathrm{T}$ cells, leukemia patients rapidly entered remission and remain disease-free due to generation of long-lived memory $T$ cells $[8,9]$. Because their $T$ cells were genetically engineered against all $\mathrm{CD} 19^{+}$cells, both normal and leukemic B cells were eliminated. Although tumor cells initially trigger an immune response [10], they suppress immune cell activity 
by engaging coinhibitory receptors including Tim-3, programmed cell death protein 1 (PD1), and cytotoxic T-lymphocyte-associated antigen 4 (CTLA-4) as well as by secreting immunosuppressive factors such as transforming growth factor beta (TGF- $\beta$ ) [11]. Fortunately, cancer-impaired immune cells can be repaired and activated in vitro [12] and in vivo - without genetic engineering - as demonstrated by potent stimulation of tumorreactive $T$ cells in metastatic melanoma patients treated with ipilimumab to block CTLA4 [13].

In the setting of AML, Glaser and colleagues [14] have shown that in vitro manipulation of AML cells provides relevant insights and predicts promising therapeutic effects in vivo. Furthermore, Noh and colleagues [15] greatly improved survival of mice with colon cancer using a two-pronged immune strategy inspired by in vitro study of tumor cells that were not conventional cancer stem cells (CSCs), but stem-like surrogates. By decreasing tumor cell resistance to immune attack, adding a Nanog inhibitor to antigenspecific cytotoxic T cell therapy markedly improved efficacy [15]. In the future, in vitro studies of a patient's tumor and immune cells may help predict the effectiveness of candidate therapies if the diversity of AML subtypes requires patient-specific treatments.

Although LPC surface antigens such as CD123 [16], CD47 [17], and Tim-3 [3, 4] are shared by subsets of AML patients, each patient's LPCs are genetically unique and no universal LPC markers have been identified. Thus, patient-specific therapies may be necessary, and some have saved the lives of leukemia patients with no other options [8, 9]. Engineering patient-specific immune therapies may require insight into how similar the primary cells cultured in vitro must be to the LPCs evolving in vivo in order to serve as relevant therapeutic targets. Presumably, when engineering antibodies and/or 
activated antigen-specific immune cells, the greater the similarity, the greater the likelihood of efficacy. Although LPCs are continually evolving in vivo, working with stemlike tumor cells (instead of true LPCs) in vitro may be adequate for developing immune therapies because of immunologic cross-reactivity.

Unfortunately, the same LPCs that are hard to eliminate in vivo (MRD cells) are difficult to maintain in vitro. In culture, LPCs tend to rapidly die or differentiate into more mature, less relevant cells with phenotypes that may differ from tumor cells that sustain disease and trigger relapse. For the culture of primitive cancer cells, prior technical advances provide guidance. Physiologic $\mathrm{BM}$ oxygen levels $\left(1-5 \% \mathrm{O}_{2}\right)$ [18], hematopoietic cytokines, and BM stromal cells improve survival of AML progenitors [19]. In mammosphere [20] and neurosphere [21] assays, 3-D architecture helps maintain and expand CSCs, illustrating the diversity of signals that influence CSCs.

To address the challenge of maintaining LPCs in vitro, we first screened nongenetic factors that might slow decay of $\mathrm{CD} 34^{+} \mathrm{AML}$ cells. In general, a patient's CD $34^{+}$ AML cells may include bona fide disease-causing LPCs [22], primitive AML cells that are similar enough to LPCs to be useful for engineering immune therapies, or AML cells that need to be reprogrammed toward a more stem-like phenotype in order to serve as relevant therapeutic targets $[15,23]$. Unfortunately, a patient's most recently evolved and lethal disease-causing LPCs may be impossible to identify. Despite an evolving therapeutic target, engineering of patient-specific immune therapies will likely advance by extending in vitro survival of primitive AML cells as well as normal immune cells and HSCs. Prolonging in vitro survival will facilitate exploring how to increase LPC immunogenicity, repair and activate immune cells, improve antigen presentation, and 
engineer LPC-targeted therapies. Fortunately, prior studies show in vitro results can predict in vivo effects despite the differences in these environments [13, 14].

To better maintain primary $\mathrm{CD} 34^{+} \mathrm{AML}$ cells, we screened factors including low $\mathrm{O}_{2}$, co-culture with BM stromal cells, and compounds that agglutinate cells, affect TGF$\beta$, or promote stem cell maintenance. Along with low $\mathrm{O}_{2}$ and co-culture with $\mathrm{BM}$ stromal cells, RepSox, a chemical reprogramming tool [24] and ATP-competitive inhibitor of TGF$\beta$ receptor 1 (TGF- $\beta$ R1) kinase [25], attracted our attention because it slowed decline of $\mathrm{CD}_{34}{ }^{+} \mathrm{AML}$ and myelodysplastic syndrome (MDS) cells in culture. Of clinical relevance, CD34 ${ }^{+} \mathrm{AML}$ cells often contain disease-causing LPCs that may trigger relapse [22]. 


\section{Materials and Methods}

\section{Human WBCs}

De-identified leukapheresis specimens were obtained from patients treated at the West Virginia University (WVU) Mary Babb Randolph Cancer Center in accordance with Institutional Review Board guidelines. WBCs were isolated by Ficoll-Paque Plus (Amersham Biosciences, Uppsala, Sweden) density gradient separation and cryopreserved $\left(5 \times 10^{6}\right.$ cells $\left./ \mathrm{mL}\right)$ in equal volumes of RPMI 1640 medium $/ 10 \%$ fetal bovine serum (FBS) and freezing solution ( $80 \% \mathrm{FBS} / 20 \%$ dimethyl sulfoxide or DMSO). $\mathrm{CD}^{+} 4^{+}$ cells isolated from the mobilized peripheral blood of healthy donors were purchased from AllCells (Emeryville, CA).

\section{Cell culture}

WBCs were cultured in RPMI 1640 medium (Mediatech, Manassas, VA) containing 2 mM L-glutamine (Mediatech), $100 \mathrm{U} / \mathrm{mL}$ penicillin, $0.1 \mathrm{mg} / \mathrm{mL}$ streptomycin (Sigma-Aldrich, St Louis, MO), and 10\% FBS (Hyclone, Logan, UT) or serum replacement 1 (Sigma) (also referred to as serum-free) as indicated. Co-cultures included BM stromal cells, adherent cells isolated from the BM of patients without evidence of hematologic disease. BM stromal cells constitutively express VCAM-1, fibronectin, and diverse cytokines that support hematopoietic progenitors, including IL-7 dependent pro-B cells [26]. Cells were cultured at $37^{\circ} \mathrm{C}$ in $6 \% \mathrm{CO}_{2}$ and $1-5 \%$ or $21 \% \mathrm{O}_{2}$ as noted.

\section{TGF- $\beta$ inhibition and neutralization}

TGF- $\beta$ inhibitors RepSox (E-616452 or SJN 2511; Sigma), SB431542 (Sigma), LY364947, and GW788388 (Tocris Bioscience, Ellisville, MO) were dissolved in DMSO 
and added once at initiation of culture. TGF- $\beta$ neutralizing antibody (anti-TGF- $\beta 1 / 2 / 3$ ) or matched mouse IgG1 isotype control antibody (R\&D systems, Minneapolis, MN) were reconstituted in PBS. Antibodies $(10 \mu \mathrm{g} / \mathrm{mL})$ were added at initiation of culture and subsequently every 3 days.

\section{Surface immunostaining}

WBCs were suspended in $100 \mu \mathrm{L}$ phosphate buffered saline (PBS)/1\% bovine serum albumin (BSA). Samples were blocked with $1 \mu \mathrm{g}$ human IgG (R\&D systems) for 20 minutes at $4^{\circ} \mathrm{C}$ and incubated with specific or isotype control antibodies for 20 minutes at $4^{\circ} \mathrm{C}$ in the dark. Samples were rinsed 3 times with PBS $/ 1 \%$ BSA and analyzed by flow cytometry within 4 hours of staining. Specific and matched isotype control antibodies directed against the following human antigens were used: CD34-PerCP (Becton

Dickinson, San Jose, CA) and mouse IgG1-PerCP (Dako, Carpinteria, CA); HLA-A/B/CPE and mouse IgG1,K-PE; CD47-FITC and mouse IgG1,K-FITC, CD227/MUC1-FITC and mouse IgG1,K-FITC (BD Biosciences, San Jose, CA); and Tim-3-PE and mouse IgG1,KPE (BioLegend, San Diego, CA). Of note, Aldefluor-stained cells were immunostained in Aldefluor assay buffer, which contains ABC-transport inhibitors, to prevent dye efflux.

\section{Isolation of RNA and RT-PCR}

RNA was isolated from AML cells using the RNeasy Mini Kit with on-column DNase I digestion (Qiagen, Valencia, CA). One-step RT-PCRs were performed in triplicate using $50 \mathrm{ng}$ of RNA per reaction with the QuantiTect SYBR Green RT-PCR kit (Qiagen) on an Applied Biosystems 7500 thermocycler (Foster City, CA). GUSB served as a loading control. Primers for human CD34, CXCL12, MYC, and HAVCR2 (encoding Tim-3) were purchased from Qiagen. GUSB primers (forward: AAACGATTGCAGGGTTTCAC, 
reverse: CTCTCGTCGGTGACTGTTCA) were synthesized by Invitrogen (Carlsbad, CA). Fold changes in relative gene expression were calculated using the $2^{-\Delta \Delta} C_{\top}$ method [27].

\section{Carboxyfluorescein diacetate, succinimidyl ester (CFSE) staining}

WBCs were labeled using the CellTrace CFSE cell proliferation kit (Molecular Probes, Eugene, OR) according to manufacturer's instructions. Briefly, WBCs were incubated with $1 \mu \mathrm{M}$ CFSE for 10 minutes at $37^{\circ} \mathrm{C}$. Staining was quenched using ice-cold media, and cells were rinsed and placed in culture. After six days, cells were analyzed by flow cytometry.

\section{Aldefluor assay}

Aldehyde dehydrogenase (ALDH) activity was measured using the Aldefluor assay according to manufacturer's instructions (Aldagen, Durham, NC). WBCs were suspended in Aldefluor assay buffer and incubated with Aldefluor reagent, a fluorescent substrate of ALDH. An aliquot of each sample was immediately mixed with diethylaminobenzaldehyde (DEAB), a specific inhibitor of $A L D H$, to serve as a negative control. $\mathrm{ALDH}^{+}$regions were drawn to exclude $>99 \%$ of DEAB-treated cells. Samples were incubated for 40 minutes at $37^{\circ} \mathrm{C}$ and analyzed by flow cytometry within 3 hours of labeling.

\section{Flow cytometric analysis}

Samples were analyzed by fluorescence-activated cell sorting (FACS) using a Becton Dickinson FACSCalibur. Data were analyzed using FCS Express software (De Novo Software, Los Angeles, CA). Dead cells, identified by propidium iodide uptake, were excluded from analysis. The Overton histogram subtraction method was used to calculate the percentage of stained (i.e. CD34 ${ }^{+}$) cells relative to matched isotype controls 
[28]. Normalized median fluorescence intensity (MFI) was calculated by subtracting the MFI of isotype control antibody-stained cells from the MFI of specific antibody-stained cells.

\section{Immunomagnetic sorting of CD34+ WBCs}

WBCs were co-cultured with $\mathrm{BM}$ stromal cells under $5 \% \mathrm{O}_{2}$ for $4-8$ hours after thawing. Following recovery, WBCs were labeled with magnetic beads conjugated to anti-CD34 antibody recognizing the QBEND/10 epitope (Miltenyi Biotec, Bergisch Gladbach, Germany). WBCs were separated using a Miltenyi Biotec autoMACS according to manufacturer's instructions. Purity of cellular fractions was evaluated by flow cytometry following immunostaining with anti-CD34 antibody recognizing the HPCA-2 epitope (Becton Dickinson).

\section{FACS of Tim $-3^{+}$WBCs}

WBCs were co-cultured with $\mathrm{BM}$ stromal cells under $5 \% \mathrm{O}_{2}$ for $4-8$ hours after thawing. Following recovery, WBCs were stained with anti-Tim-3-PE antibody (BioLegend) and sorted into Tim $-3^{+}$and Tim- $3^{-}$fractions (> $88 \%$ pure) using a Becton Dickinson FACSAria and FACSDiva 6.2 software. Tim- $3^{+}$cells were gated against matched isotype control antibody-stained cells. Purity of sorted fractions was evaluated by flow cytometry.

\section{Colony-forming assays}

WBCs were cultured in methylcellulose containing recombinant human stem cell factor (SCF), granulocyte-macrophage colony stimulating factor (GM-CSF), granulocyte colony stimulating factor (G-CSF), interleukin-3 (IL-3), and erythropoietin (Epo) (MethoCult Optimum, Stem Cell Technologies, Vancouver, British Columbia, Canada) according to manufacturer's instructions. Colony assays, performed in duplicate, were incubated at 
$37^{\circ} \mathrm{C}$ in $6 \% \mathrm{CO}_{2}$ and $21 \% \mathrm{O}_{2}$. Colonies were counted by light microscopy after $14-18$ days of culture.

\section{Diff-Quik staining}

To evaluate morphology, WBCs were stained using the Diff-Quik Stain Set (Siemens, Newark, DE) according to manufacturer's instructions. Briefly, slides were sequentially dipped in methanol fixative, a solution of Eosin $\mathrm{Y}$, and a solution containing methylene blue and Azure A. Slides were rinsed with deionized water, allowed to dry, and imaged by light microscopy under oil immersion.

\section{Fluorescence in situ hybridization (FISH)}

WVU Hospital Cytogenetics Laboratory analyzed 100-150 interphase cells for $t(8 ; 21)$, $\mathrm{t}(15 ; 17)$, inv(16), del(6q23), and 11q23 chromosomal abnormalities by FISH. Probes were designed by Abbott Molecular (Des Plaines, IL).

\section{Statistical analysis}

Differences between groups were compared using paired Student $t$ tests (2-tailed) and considered statistically significant when $P<0.05$. Values are displayed as the mean \pm standard error (SE). With the exception of $\mathrm{FISH}$, all data are representative of $\geq 2$ independent experiments. 


\section{Results}

CD34 ${ }^{+}$AML/MDS cells have greater colony-forming and ALDH activity than CD34cells

Leukapheresis specimens (Table S1) from AML/MDS patients (P1-8) displayed variable CD34 expression (Figure 1A). To identify LPC-enriched fractions, we compared the morphology, colony-forming potential, and ALDH activity of $\mathrm{CD}^{-} 4^{+}$and CD34- cells. Following immunomagnetic sorting, CD34+ fractions were $75-99 \%$ pure (Figure S1) and 99-100\% leukemic by FISH (Figure 1E). CD34+ AML cells (P1) exhibited rounder nuclei and a higher nuclear-to-cytoplasmic ratio than CD34- AML cells (Figure 1B). CD34 ${ }^{+}$ AML/MDS cells generated 35- to 65-fold more colonies (Figure 1C) and generally displayed greater ALDH activity (Figure 1D; Figure S2) than CD34- cells. Of interest, the relapsed AML patient (P2) had the highest proportion (85\%) of $C D 34^{+}$cells with $A L D H$ activity. Colonies generated by $\mathrm{CD} 34^{+}$cells were confirmed to be of leukemic origin by FISH (Figure 1F). In culture, CD34+AML/MDS cells gave rise to CD34- cells, while CD34cells remained CD34- (Figure S3). $\quad \mathrm{CD}^{-} 4^{+}$cells also expanded via self-renewal upon stimulation with SCF, G-CSF, and IL-3 (Figure S4A). In summary, AML/MDS progenitors are enriched within $\mathrm{CD} 34^{+}$fractions of leukapheresis specimens.

\section{RepSox, low $\mathrm{O}_{2}$, and co-culture with $\mathrm{BM}$ stromal cells maintain $\mathrm{CD} 34^{+} \mathrm{AML}$ cells}

Serum-free medium (Figure 2A), co-culture with BM stromal cells (Figure 2B), and low (1$5 \%) \mathrm{O}_{2}$ (Figure 2C) more effectively maintained CD34+ $\mathrm{AML}$ cells than serum-containing medium, culture without $\mathrm{BM}$ stromal cells, and high $(21 \%) \mathrm{O}_{2}$. These conditions mimic in vitro assays designed to support LPCs [19] and the BM niche where LPCs reside in vivo 
$[18,29]$. To generate 3-D spheroids, leukemic cells were co-cultured with BM stromal cells or osteoblasts on low attachment plates (Figure S5).

Under all culture conditions examined, RepSox maintained higher proportions of $\mathrm{CD}^{+}{ }^{+}$cells than DMSO (Figure 2A-C). As expected, RepSox inhibited TGF- $\beta$-induced phosphorylation of Smad2/3 (Figure S6). Similar proportions of $\mathrm{CD}_{3} 4^{+}$cells were maintained in media and DMSO; therefore, only DMSO controls are shown. In summary, optimal conditions for maintaining CD34 ${ }^{+} \mathrm{AML}$ cells include $1-5 \% \mathrm{O}_{2}$, co-culture with BM stromal cells, and exposure to RepSox.

\section{RepSox maintains CD34 ${ }^{+} \mathrm{AML}$ cells in a concentration-dependent manner}

Increasing concentrations of RepSox maintained higher proportions of CD34+ $\mathrm{AML}$ cells (1.6-fold and 2.3-fold increase in the proportion of CD34 ${ }^{+}$cells, $2 \mu \mathrm{M}$ and $16 \mu \mathrm{M}$ RepSox

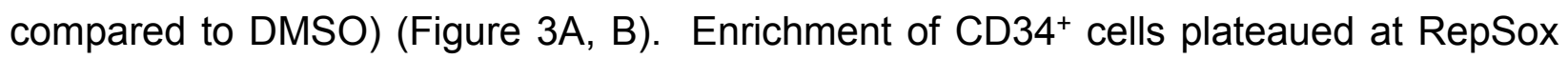
concentrations greater than $16 \mu \mathrm{M}$ (data not shown). Effects on CD34 expression were also concentration-dependent (3.0-fold and 12.5-fold increase in CD34 MFI, $2 \mu \mathrm{M}$ and $16 \mu \mathrm{M}$ RepSox compared to DMSO) (Figure 3A, C).

\section{RepSox slows decay of CD34+ AML/MDS cells}

Next we investigated the effects of RepSox on diverse AML/MDS specimens. For consistency, all specimens were cultured with $16 \mu \mathrm{M}$ RepSox, 10\% FBS, BM stromal cells, and $5 \% \mathrm{O}_{2}$. Inclusion of serum improved viability even though serum-free conditions more effectively maintained the CD $34^{+}$subset of cells.

In culture, the proportion of $\mathrm{CD} 34^{+}$AML/MDS cells declined as cells divided asymmetrically and/or differentiated. Culture with RepSox slowed the mean decline in the proportion of $\mathrm{CD}_{3} 4^{+}$cells (33\% and $54 \%$ mean decline, RepSox and DMSO, P1-7) 
(Figure 4A, B). That is, RepSox maintained 1.2 to 3.0 -fold higher proportions of CD34 ${ }^{+}$ cells than DMSO. RepSox-treated CD34 ${ }^{+}$cells were confirmed to be of leukemic origin by FISH (Figure 4C). CD34 expression was also 1.9 to 6.5-fold higher on RepSox-treated cells than DMSO-treated controls (CD34 MFls of 30 and 9, RepSox and DMSO, P1-7) (Figure 4A). RepSox did not induce CD34 expression on CD34- AML cells (Figure S3). Unlike RepSox, exposure to the TGF- $\beta$ inhibitors SB431542, LY364947, and GW788388 (Figure S7A) as well as TGF- $\beta$ neutralizing antibody (data not shown) did not slow decay of CD34+ AML cells. In contrast to AML/MDS cells, RepSox did not slow decay of CD34+ cells from healthy donors (Figure 4A, B).

We investigated whether RepSox slows decay of CD34+ AML/MDS cells by slowing their rate of asymmetric division and/or by inhibiting differentiation. RepSoxtreated AML cells retained greater CFSE fluorescence (Figure 4D) and incorporated less EdU (Figure S8) than DMSO-treated controls, consistent with a slower rate of proliferation. Upon stimulation with IL-3, SCF, and G-CSF, RepSox-treated CD $34^{+}$cells expanded as effectively as DMSO-treated controls (Figure S4A). We also evaluated how RepSox may affect differentiation of $\mathrm{CD} 34^{+}$cells into CD34- $\mathrm{AML}$ cells. When cultured with DMSO, the proportion of $\mathrm{CD} 34^{+} \mathrm{AML}$ cells (P1) decreased as the proportion of CD34CD33-CD14- cells increased (data not shown). In contrast, RepSox maintained a high proportion of $\mathrm{CD}_{3} 4^{+}$cells, and no increase in the proportion of CD34-CD33-CD14- cells was observed (data not shown). 


\section{RepSox-treated AML/MDS cells display higher CD34, CXCL12, and MYC mRNA levels and similar ALDH activity compared to DMSO-treated controls}

Both c-Myc and C-X-C motif chemokine 12 (CXCL12)/stromal cell-derived factor 1 (SDF-1) may inhibit differentiation of AML cells [30, 31, 32, 33]. RepSox-treated AML cells displayed higher CD34, CXCL12, and MYC mRNA levels than DMSO-treated controls (Figure 5A). CXCL12 and MYC mRNA levels were substantially higher following exposure to RepSox than the TGF- $\beta$ inhibitors SB431542 and LY364947 (Figure S7C).

ALDH is involved in chemotherapy resistance [34] and normal HSC self-renewal [35]. In AML patients, the subset of $\mathrm{CD} 34^{+}$cells with ALDH activity is enriched in longterm culture-initiating cells [36]. Furthermore, persistence of $C D 34^{+} \mathrm{CD} 38-\mathrm{ALDH}{ }^{\text {int }} \mathrm{AML}$ cells after induction chemotherapy is associated with disease relapse [37]. Given this clinical relevance, we explored whether RepSox-treated CD34+ cells retain ALDH activity. Similar proportions of RepSox- and DMSO-treated CD34+ cells (gated as shown in Figure S9) displayed ALDH activity (Figure 5B), demonstrating RepSox equally slows decay of $\mathrm{ALDH}^{+}$and $\mathrm{ALDH}{ }^{-}$subsets of $\mathrm{CD} 34^{+}$cells. Overall, a subset of $\mathrm{CD} 34^{+}$cells with $\mathrm{ALDH}$ activity is maintained in our culture system. The observed pattern of ALDH activity is characteristic of leukemic rather than normal stem/progenitor cells [38].

\section{RepSox reversibly suppresses AML colony-forming activity}

In addition to CD34 expression and ALDH activity, we compared the colony-forming activity (CFA) of RepSox- and DMSO-treated AML/MDS cells. Normal and AML CD34+ cells generated fewer colonies after 6-day culture with RepSox than with DMSO (Figure 6A). RepSox did not alter the low CFA of CD34- AML/MDS cells (data not shown). CFA of normal and AML CD34+ cells was reduced with RepSox compared to DMSO included 
in the colony-forming assay (Figure 6B). In contrast, CFA of CD34 ${ }^{+} \mathrm{MDS}$ cells was higher with RepSox compared to DMSO included in the colony-forming assay (Figure 6B).

Potential reasons for the reduced CFA of AML cells after RepSox exposure include death, differentiation, or arrest of colony-forming cells in a quiescent stem-like state. To rule out RepSox-induced death or differentiation of colony-forming cells, we measured CFA following RepSox removal. Removal of RepSox partially rescued AML CFA (Figure $6 C)$.

\section{RepSox decreases Tim-3 expression on AML/MDS cells}

Increasing tumor cell immunogenicity is one strategy to improve immune cell activation [6]. Therefore, we investigated whether RepSox alters expression of immunomodulatory receptors including Tim-3, CD47, human leukocyte antigen (HLA), and mucin-1 (MUC1) on AML/MDS cells. HAVCR2 (encoding Tim-3) mRNA levels were consistently reduced in RepSox-treated AML cells compared to DMSO-treated controls (Figure 7A). A smaller proportion of RepSox-treated CD34 ${ }^{+}$cells expressed Tim-3, a negative regulator of antitumor immunity [39], than DMSO-treated CD $34^{+}$cells (mean $27 \%$ and $53 \%$ Tim- $3^{+}$cells, RepSox and DMSO, P2-3) (Figure 7B). To determine whether RepSox promoted selective survival of Tim- $3^{-}$cells or decreased Tim-3 expression, Tim- $3^{+}$and $\mathrm{Tim}-3^{-}$ fractions were separately exposed to RepSox. Tim $-3^{+}$cells converted to Tim-3- cells in vitro (Figure 7C). RepSox accelerated loss of Tim-3 from the surface of FACS-purified Tim- $3^{+}$AML/MDS cells ( $64 \%$ and $43 \%$ mean decline in Tim $-3^{+}$cells, RepSox and DMSO, P2-3 and P8) (Figure $7 \mathrm{C}$ ). A greater proportion of MDS cells expressed Tim-3 following removal from RepSox (Figure S10A) compared to continuous culture with RepSox (Figure S10B), suggesting Tim-3 effects are partially reversible. Exposure to both RepSox and 
the structurally distinct TGF- $\beta$ inhibitor SB431542 [40] accelerated loss of Tim-3 from the surface of AML/MDS cells ( $81 \%$ and $53 \%$ mean decline in Tim- $3^{+}$cells, SB431542 and DMSO, P3 and P8) without altering viability relative to controls (Figure S11). Expression of CD47 and HLA-A/B/C was not affected by RepSox, while MUC1, an antigen contributing to the poor immunogenicity of AML LPCs [41], was not highly expressed by AML/MDS cells (Figure S12). 


\section{Discussion}

Engineering patient-specific anti-cancer immune therapies will advance as in vitro techniques improve. Because primitive relapse-causing tumor cells must be targeted, a basic challenge is reducing the in vitro death and differentiation of cancer progenitors. LPCs must be maintained in culture long enough to study their molecular characteristics, activate immune cells, and/or evaluate potential therapies. Currently, key technical obstacles are being identified and addressed. Glettig and Kaplan have explored ways to better maintain $\mathrm{CD} 34^{+}$hematopoietic progenitors in vitro using co-culture with adipocytes [42]. Kellner and colleagues report their "most significant problem" is isolating multiple myeloma stem cells so they can be studied [43]. If relevant stem-like tumor cells can be adequately maintained in vitro, another challenge may be increasing low tumor cell immunogenicity that impedes antigen presentation and immune cell activation. In the setting of AML, abnormal Tim-3 expression on tumor cells and dysregulated antigenpresenting and immune-effector cells is an especially attractive target because this one receptor impairs antigen presentation and promotes both immune suppression and immune evasion $[44,45,46,47]$. Thus, Tim-3 should be considered a potentially serious obstacle when engineering immune therapies in vitro.

In light of these technical challenges, the ability of RepSox to prolong $\mathrm{CD} 34^{+}$cell survival and decrease Tim-3 expression seems useful for engineering anti-LPC immune therapies. RepSox is a chemical reprogramming tool with actions linked to maintaining stemness and inhibiting differentiation: two actions relevant for maintaining CD34+ $\mathrm{AML}$ cells in culture $[24,48]$. In mouse embryonic fibroblasts (MEFs), RepSox increased mRNA levels of components of the Wnt, Notch, and Hedgehog signaling pathways that 
promote pluripotency, self-renewal, and stem cell maintenance [48]. Also, as a TGF- $\beta$ inhibitor, RepSox may mitigate immunosuppressive effects induced by TGF- $\beta$ during cancer progression [49]. Thus, RepSox may be a valuable tool for manipulating cancer and immune cells because its actions on both normal and cancer cells are potent and consistent [24, 48].

In addition to their poor survival and low immunogenicity, patients' tumor cells may not be stem-like enough to adequately represent the critical in vivo target: the most recently evolved LPCs capable of triggering relapse. Thus, primary cells may need to be reprogrammed toward more stem-like phenotypes in order to serve as relevant therapeutic targets. Fortunately, technical breakthroughs are encouraging. The same factors that dedifferentiate normal cells may also dedifferentiate tumor cells $[15,23,50]$. Noh converted tumor cells into a more stem-like phenotype in vitro after identifying a mechanism by which immune-selection pressures drove this conversion in vivo [15]. Furthermore, mouse cells were chemically reprogrammed to pluripotency using seven small molecules [51].

In this study, the TGF- $\beta$ inhibitors RepSox and SB431542 reduced Tim-3 expression on AML cells. However, only RepSox-treated AML cells displayed substantially increased levels of CXCL12 and MYC mRNA, and only RepSox slowed decay of CD34 ${ }^{+} \mathrm{AML}$ cells. Slowing decay of $\mathrm{CD} 34^{+}$cells is consistent with prior RepSox findings: RepSox replaces c-Myc during reprogramming [24], RepSox upregulates CXCL12 and MYC in MEFs [48], and both c-Myc and CXCL12 promote tumor cell survival [32, 52, 53, 54]. Furthermore, c-Myc is downregulated during AML cell differentiation [31], and exposing AML cells to a c-Myc inhibitor induces differentiation [33]. 
The CXCL12/CXCR4 and/or CXCL12/CXCR7 axes promote survival of LPCs and normal CD $34^{+}$cells $[55,56]$. Activation of the CXCL12/CXCR4 axis is thought to inhibit AML cell differentiation because inhibition of CXCR4 by AMD3100 induces AML cells to differentiate [32]. Overall, CXCL12 promotes survival of leukemic progenitors both in vitro and in vivo $[57,58]$. However, the implications of cell surface and intracellular receptor levels are still being clarified. In the case of CXCR7, the CXCL12/CXCR7 axis promotes HSC survival even when CXCR7 surface expression is scarce [56]. Conceivably, when used as a potential CXCL12 upregulator, RepSox might help clarify the role of intracellular CXCL12 levels. Of note, the balance of CXCL12- and TGF- $\beta$-activated pathways affects $\mathrm{CD}_{3} 4^{+}$cell cycle status [59]. Therefore, as both a TGF- $\beta$ inhibitor and an apparent CXCL12 upregulator, RepSox seems useful for investigating the balance between quiescence and cell cycling.

RepSox may slow decay of CD34+ AML cells by increasing c-Myc which inhibits differentiation. c-Myc blocks differentiation of AML cell lines by increasing microRNA-17 and microRNA-20a levels which, in turn, decrease p21 and STAT3 [60]. RepSox may increase c-Myc by upregulating CXCL12 which signals via CXCR4 to activate NF-KB [61]. NF-KB can increase expression of microRNA binding protein Lin28 [62] which inhibits maturation of the let-7 family of microRNAs $[62,63]$. Because let-7 microRNAs repress translation of MYC mRNA, reduced let-7 may lead to increased c-Myc expression [63, 64]. Furthermore, RepSox-treated MEFs expressed higher mRNA levels of Wnt signaling components $F Z D 1, F Z D 4, F Z D 9$, and $L E F 1$ than DMSO-treated controls [48]. Because MYC is a target of Wnt signaling [65], RepSox may upregulate MYC via Wnt activation. Upregulation of MYC by $\mathrm{Wnt} / \beta$-catenin signaling has been reported independently by 
Zhang [66] and He [65], and He highlighted the T cell factor-4 binding sites in the MYC promoter. CXCL12 exposure also increased mRNA levels of the Wnt target genes CTNNB1 (encoding $\beta$-catenin), CCND1 (encoding cyclin-D1), and MYC (encoding c-Myc) in the AML cell line HL60 [67].

In contrast to the "CD34+ effect" unique to RepSox, Tim-3 reduction on AML/MDS cells was reproduced by the structurally distinct TGF- $\beta$ inhibitor SB431542. Thus, TGF$\beta$ inhibition may reduce Tim-3 levels, a mechanism supported by reports that TGF- $\beta$ induces Tim-3 expression on mast cells $[68,69]$. Of note, the ALDH activity of RepSoxtreated $\mathrm{CD} 34^{+} \mathrm{AML}$ cells did not change, despite reduced Tim- 3 expression, suggesting these cells were still stem-like. Although Tim-3 is an AML LPC marker, reduced Tim-3 expression may not diminish the disease-initiating or relapse-causing potential of LPCs. Indeed, RepSox-treated AML cells engrafted the BM of NOD-scid IL2Rgammanull mice (data not shown).

Tim-3 may be a critical obstacle when engineering AML immunotherapies because it impairs anti-tumor immunity [39]. By increasing Tim-3 expression, tumor microenvironments restrain the anti-tumor activity of dendritic [70], NK [71], T-lymphocytic [39], and monocytic [72] cells. In turn, blocking Tim-3 signaling with antibodies restores the cytotoxicity of $\mathrm{CD}^{+} \mathrm{T}$ cells and improves anti-tumor immunity $[12,73,74]$. Conceivably, reducing Tim-3 expression on AML cells might increase their immunogenicity because Tim-3 binds to, and suppresses, $T_{H}$ cells when expressed by endothelial cells [47].

Immunotherapies involving antibodies, activated immune cells, and/or inhibitors of anti-apoptotic factors (responsible for tumor cell immune resistance) may be required to 
eliminate LPCs that trigger AML relapse. Although a cancer patient's immune-effector and antigen-presenting cells are not malignant, their cancer-induced dysregulations may need to be reversed. Reprogramming exhausted $T$ cells to pluripotency reactivates their function, increases their proliferative capacity, and preserves their antigen-specificity upon subsequent re-differentiation [75, 76]. Because immune cell suppression and tumor cell immune-evasion worsen over time, immune therapies may need to be administered shortly after diagnosis to be effective. Thus, the ability to quickly manipulate a patient's diagnostic cells could be an advantage. Like N-propionylmannosamine [77], resiquimod [78], and the MUC1 inhibitor GO-203 [41], RepSox is a chemical that alters leukemia cells in vitro within a week. Prolonging survival of a patient's CD34 ${ }^{+}$cells in culture by days may be sufficient to chemically engineer cells. Exposing cells to small molecules like RepSox is less labor-intensive than genetic engineering and effects may be reversible. Of note, culture with a leukemic patient's BM stromal cells, rather than normal BM stromal cells, may further enhance maintenance of that patient's CD34 ${ }^{+} \mathrm{AML}$ cells because cancer-associated fibroblasts have evolved to support tumor growth in vivo $[79,80]$. The ability to quickly and reversibly alter the primary cells of AML patients in vitro may be valuable if sequential cellular manipulations prove useful for therapy development. 


\section{Conclusion}

RepSox may be useful for developing immunotherapies as a cell culture additive (to slow decay of $\mathrm{CD}_{3} 4^{+} \mathrm{AML}$ cells which often contain LPCs) and/or as a cell-engineering tool (to decrease Tim-3 expression). Because Tim-3 expression on the tumor and immune cells of cancer patients may adversely affect tumor cell immunogenicity, immune cell activation, and antigen presentation, RepSox may improve the anti-tumor actions of antigen-presenting and immune-effector cells that become dysregulated in cancer patients. Since the effects of RepSox seem potent and predictable, RepSox warrants consideration for manipulating the tumor and immune cells of patients with other cancers. Because molecular tools can chemically reprogram cells, RepSox and other molecules might eliminate the need for genetic engineering when developing patient-specific immune therapies. Characterizing the actions of RepSox and other tools may promote development of immunotherapies by simplifying in vitro engineering of tumor, antigenpresenting, and immune-effector cells. 


\section{Acknowledgements}

This work and LFG was supported by the National Institutes of Health (NIH) grants RO1 HL056888 and RO1 CA134573, NIGMS P30 GM103488, NIGMS CTR-IDeA U54GM104942, the Alexander B. Osborn Hematopoietic Malignancy and Transplantation Program, and the West Virginia Research Trust Fund. AJ was supported by a National Cancer Institute (NCl) pre-doctoral fellowship F31 CA159805 and an $\mathrm{NCl}$ supplement R01 CA134573-02S1. We thank the West Virginia University (WVU) Tissue Bank, WVU Biospecimen Processing Core, and patients and clinicians at the Mary Babb Randolph Cancer Center for donating leukapheresis specimens. We gratefully acknowledge Dr. Kathleen Brundage for expertise related to flow cytometry experiments performed in the WVU Flow Cytometry Core Facility, supported in part by an $\mathrm{NIH}$ equipment grant RR020866, NIGMS P30 GM103488, and INBRE RCP11011809. We thank the WVU Microscope Imaging Facility, which has been supported by the Mary Babb Randolph Cancer Center and NIH grants P20 RR016440, P30 RR032138/GM103488 and P20 RR016477 and the WVU non-linear optical microscopy laboratory supported by the WVU

Center for Neuroscience and CoBRE grant P30 R031155. We thank the Gibson lab and Drs. Ryan and Jessica Jajosky for their insights and advice.

Correspondence: Laura F. Gibson, P.O. Box 9300, Mary Babb Randolph Cancer Center,

1 Medical Center Drive, Morgantown, WV 26506; email: Igibson@hsc.wvu.edu; and Audrey Jajosky, P.O. Box 9300, Mary Babb Randolph Cancer Center, 1 Medical Center Drive, Morgantown, WV 26506; email: ajajosky@gmail.com 


\section{Figure legends}

Figure 1. Enrichment of colony-forming and $\mathrm{ALDH}^{+} \mathrm{AML}$ and MDS cells within CD34 $^{+}$fractions of leukapheresis specimens. (A) Flow cytometric analysis of CD34 surface expression (open histograms) on WBCs isolated from leukapheresis specimens. Solid histograms display isotype controls. (B) Diff-Quik staining of $\mathrm{CD}_{3} 4^{+}$and $\mathrm{CD} 34^{-}$cells (P1). (C) Colony-forming activity of $\mathrm{CD} 34^{+}$and $\mathrm{CD} 34^{-}$cells (mean \pm high and low counts from duplicate assays). ${ }^{*} \mathrm{P}<0.05,108 \pm 29$ and $3 \pm 1$, mean colonies generated by $\mathrm{CD}^{+} 4^{+}$and CD34 cells (P1-4). (D) Summary of the ALDH activity of CD34 ${ }^{+}$and CD34 cells from the representative experiment shown in Figure S2 (mean difference was NS). (E, F) FISH for leukemic alterations in $\mathrm{CD}^{+} 4^{+}$and $\mathrm{CD} 34^{-}$cells isolated from leukapheresis specimens as well as colonies generated by $\mathrm{CD}^{+} 4^{+}$cells. Abbreviations: NS, not statistically significant; FISH, fluorescence in situ hybridization.

Figure 2. RepSox, low $\mathrm{O}_{2}$, and co-culture with BM stromal cells maintain CD34+ AML cells. Flow cytometric analysis of CD34 surface expression (open histograms) on AML cells (P1) after 10-day culture with $16 \mu \mathrm{M}$ RepSox or DMSO (vehicle control) in the following conditions: (A) with 10\% FBS or serum replacement (off stroma, $1 \% \mathrm{O}_{2}$ ), (B) on or off BM stromal cells (serum-free, $1 \% \mathrm{O}_{2}$ ), and (C) under $1 \%$ or $21 \% \mathrm{O}_{2}$ (on stroma, serum-free). Solid histograms display isotype controls.

Figure 3. RepSox maintains $\mathrm{CD}^{+} 4^{+} \mathrm{AML}$ cells in a concentration-dependent manner. (A) Flow cytometric analysis of CD34 surface expression (open histograms) on AML cells (P1) after 10-day culture with 2-16 $\mu \mathrm{M}$ RepSox (on stroma, serum-free, 5\% O2) 
or DMSO (vehicle control). Solid histograms display isotype controls. Summary of the (B) proportion of $\mathrm{CD}_{4} 4^{+}$cells and (C) CD34 MFI of RepSox-treated cells relative to DMSO-treated controls from the representative experiment shown in part A.

Figure 4. RepSox slows decay of $\mathrm{CD}^{+} 4^{+} \mathrm{AML}$ and MDS cells. (A) Flow cytometric analysis of CD34 surface expression (open histograms) on normal donor (ND) and AML/MDS CD34+ cells after 6-day culture with $16 \mu \mathrm{M}$ RepSox or DMSO (on stroma, $10 \%$ FBS, $5 \% \mathrm{O}_{2}$ ). Solid histograms display isotype controls. (B) Summary of the decline in proportions of $\mathrm{CD} 34^{+}$cells from the representative experiment shown in part $A .{ }^{*} \mathrm{P}<$ $0.005,33 \pm 7 \%$ and $54 \pm 7 \%$, mean decline in CD34 ${ }^{+}$cells following RepSox and DMSO exposure (P1-7). (C) FISH for leukemic alterations in $\mathrm{CD}^{+} 4^{+}$cells (P1) purified by sorting after 6-day exposure to RepSox and DMSO. (D) CFSE staining profiles of RepSoxtreated (open histograms) and DMSO-treated (solid histograms) cells.

Figure 5. RepSox-treated cells have increased CD34, CXCL12, and MYC mRNA levels and similar ALDH activity compared to DMSO-treated controls. AML/MDS cells were cultured with $16 \mu \mathrm{M}$ RepSox or DMSO for 6 days (on stroma, $10 \%$ FBS, $5 \%$ $\mathrm{O}_{2}$ ). (A) qRT-PCR analysis of $C D 34\left({ }^{*} \mathrm{P}<0.05\right), \mathrm{CXCL} 12\left({ }^{*} \mathrm{P}<0.05\right)$, and $M Y C\left({ }^{*} \mathrm{P}<\right.$ 0.005) mRNA levels within RepSox-treated cells relative to DMSO-treated controls. (B) ALDH activity of RepSox- and DMSO-treated CD34+ AML/MDS cells measured by flow cytometry (mean difference was NS). $\mathrm{ALDH}^{+}$regions were drawn to exclude $>99 \%$ of DEAB-treated (negative control) cells. Abbreviations: ALDH, aldehyde dehydrogenase; NS, not statistically significant.

Figure 6. RepSox reversibly suppresses AML colony-forming activity. (A) CFA of $\mathrm{AML}$ and normal donor (ND) CD34+ cells after 6-day culture with $16 \mu \mathrm{M}$ RepSox or DMSO 
(on stroma, $10 \%$ FBS, $5 \% \mathrm{O}_{2}$ ). Mean difference between CFA of RepSox- and DMSOtreated AML cells was NS. (B) CFA of AML/MDS and ND CD $34^{+}$cells grown with $16 \mu \mathrm{M}$ RepSox or DMSO included in the colony-forming assay (added once during initiation). Mean difference between CFA of RepSox- and DMSO-treated AML cells was NS. (C) CFA of AML cells (P2) following continuous culture with RepSox for 6 days and removal of RepSox after 3 days. Bar graphs display mean colonies \pm high and low counts from duplicate assays. Abbreviations: CFA, colony-forming activity; CFU, colony-forming units; NS, not statistically significant.

Figure 7. RepSox decreases Tim-3 expression on AML and MDS cells. AML/MDS cells were cultured with $16 \mu \mathrm{M}$ RepSox or DMSO for 6 days (on stroma, $10 \%$ FBS, $5 \%$ $\mathrm{O}_{2}$ ). (A) qRT-PCR analysis of HAVCR2 (encoding Tim-3) mRNA levels within RepSoxtreated cells relative to DMSO-treated controls ( $\left.{ }^{*} \mathrm{P}<0.005\right)$. (B) Flow cytometric analysis of Tim-3 surface expression (open histograms) on RepSox- and DMSO-treated CD34 ${ }^{+}$ cells (mean difference was NS). Solid histograms display isotype controls. (C) Flow cytometric analysis of Tim-3 surface expression (open histograms) following culture of FACS-purified Tim- $3^{+}$AML/MDS cells with $16 \mu \mathrm{M}$ RepSox or DMSO for 6 days (on stroma, $10 \% \mathrm{FBS}, 5 \% \mathrm{O}_{2}$ ) (mean difference was NS). Solid histograms display isotype controls. Abbreviations: NS, not statistically significant. 
Figures

A
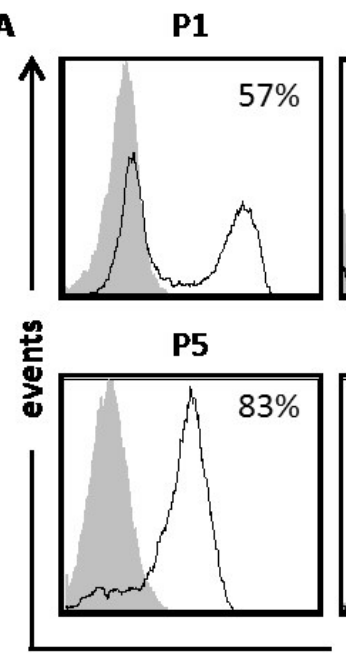

CD34 (log fluorescence intensity)

C

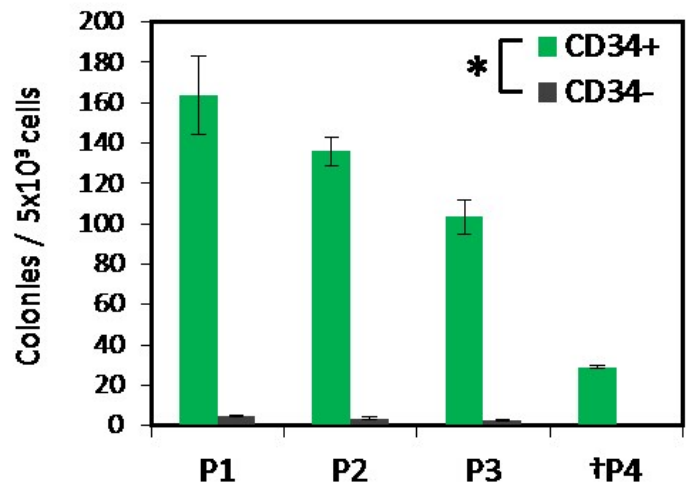

P3

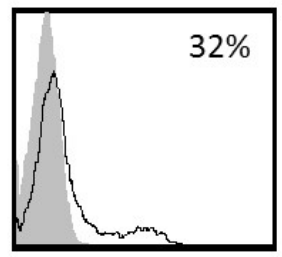

P6

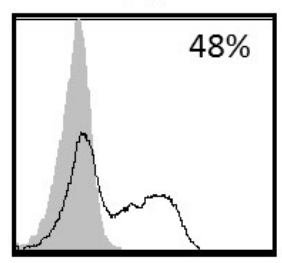

P7
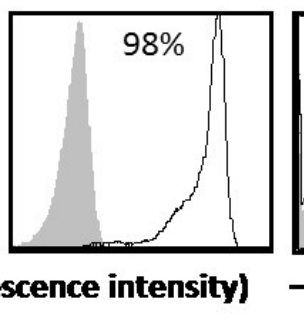

D

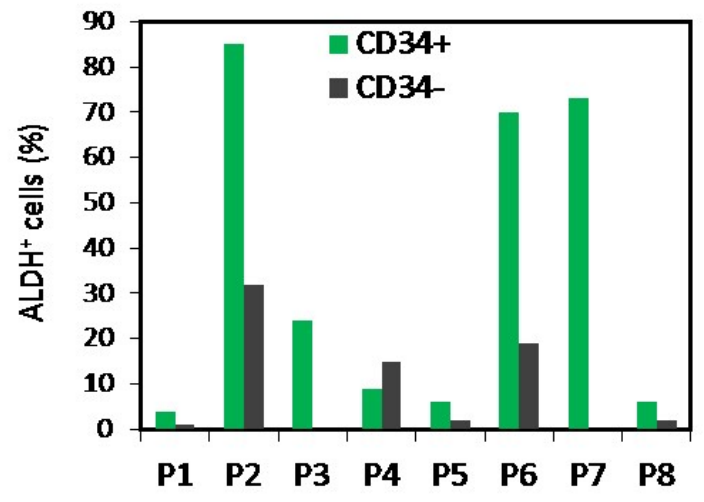

$\mathbf{E}$

\begin{tabular}{|c|c|c|}
\hline & $\begin{array}{c}\text { P1 cells with } \\
11 q 23 \\
\text { rearrangement }\end{array}$ & $\begin{array}{c}\text { P3 cells with } \\
6 q 21 \text { deletion }\end{array}$ \\
\hline${\mathrm{CD} 34^{+}}^{+}$ & $99 \%(99 / 100)$ & $100 \%(100 / 100)$ \\
\hline $\mathrm{CD} 34^{-}$ & $90 \%(90 / 100)$ & $89 \%(89 / 100)$ \\
\hline $\begin{array}{c}\text { CD34 } \\
\text { colonies }\end{array}$ & $96 \%(192 / 200)$ & $99.5 \%(199 / 200)$ \\
\hline
\end{tabular}

F $\quad \mathrm{CD}^{+}$colonies with 11q23 translocation (P1)

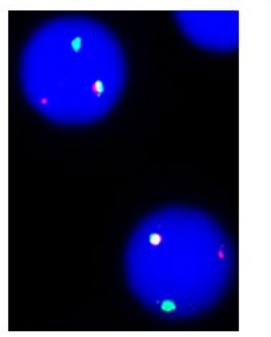

B CD34+ cells

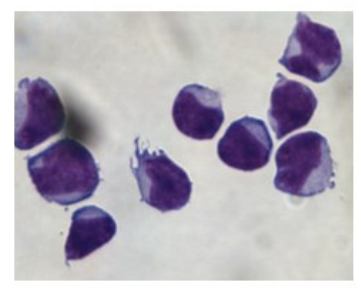

CD34 cells
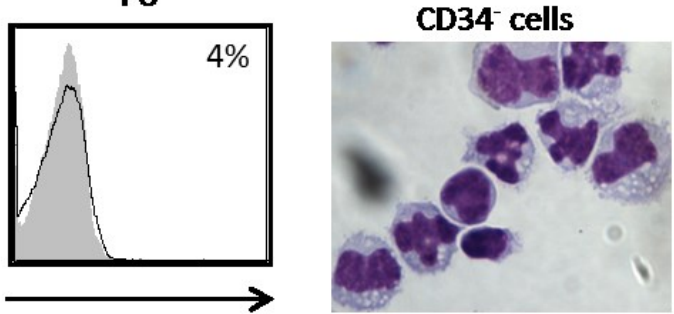

Figure 1. Enrichment of colony-forming and $A L D H^{+} A M L$ and MDS cells within $\mathrm{CD}^{+}{ }^{+}$fractions of leukapheresis specimens. 


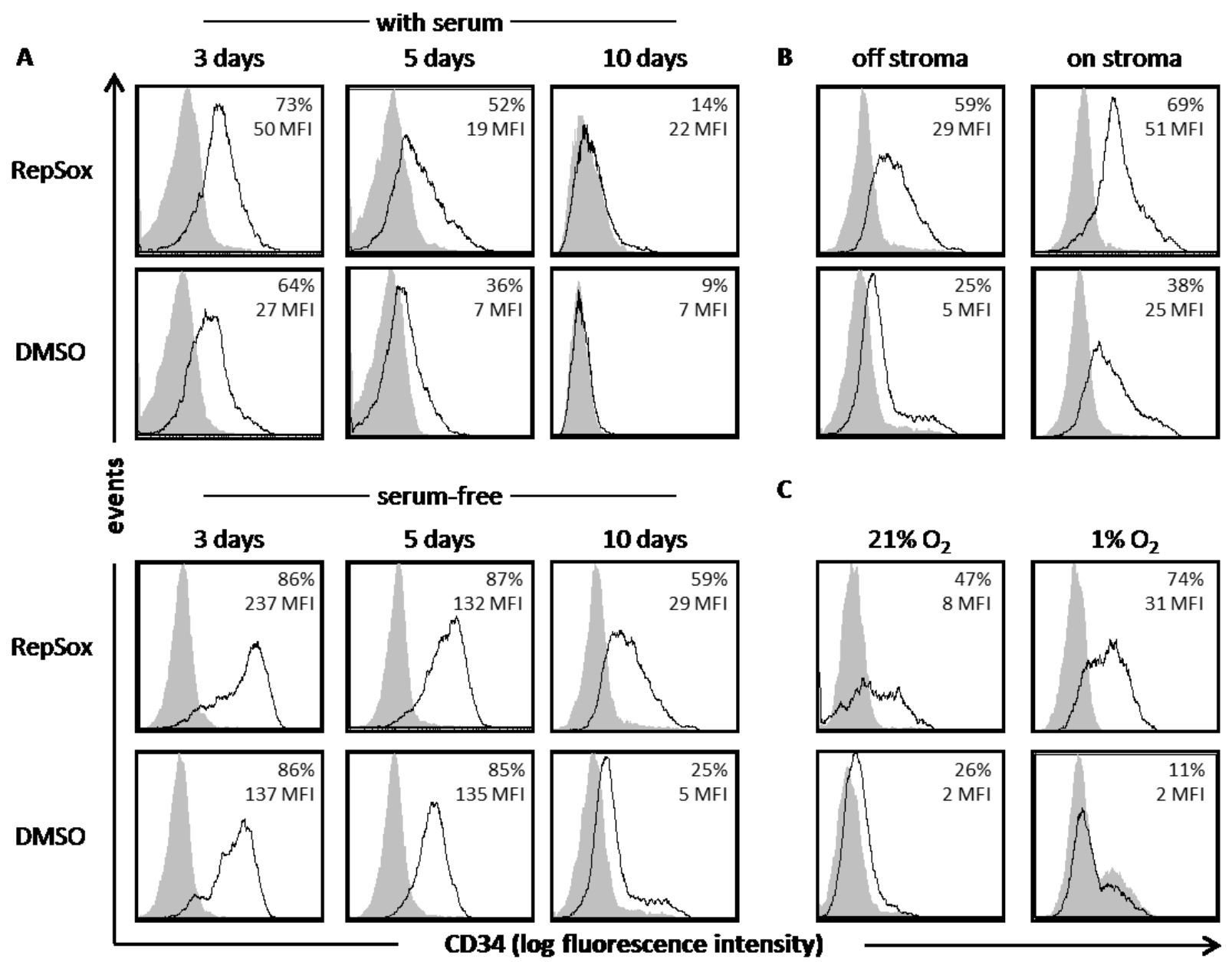

Figure 2. RepSox, low $\mathrm{O}_{2}$, and co-culture with $\mathrm{BM}$ stromal cells maintain $\mathrm{CD} 34^{+} \mathrm{AML}$ cells. 


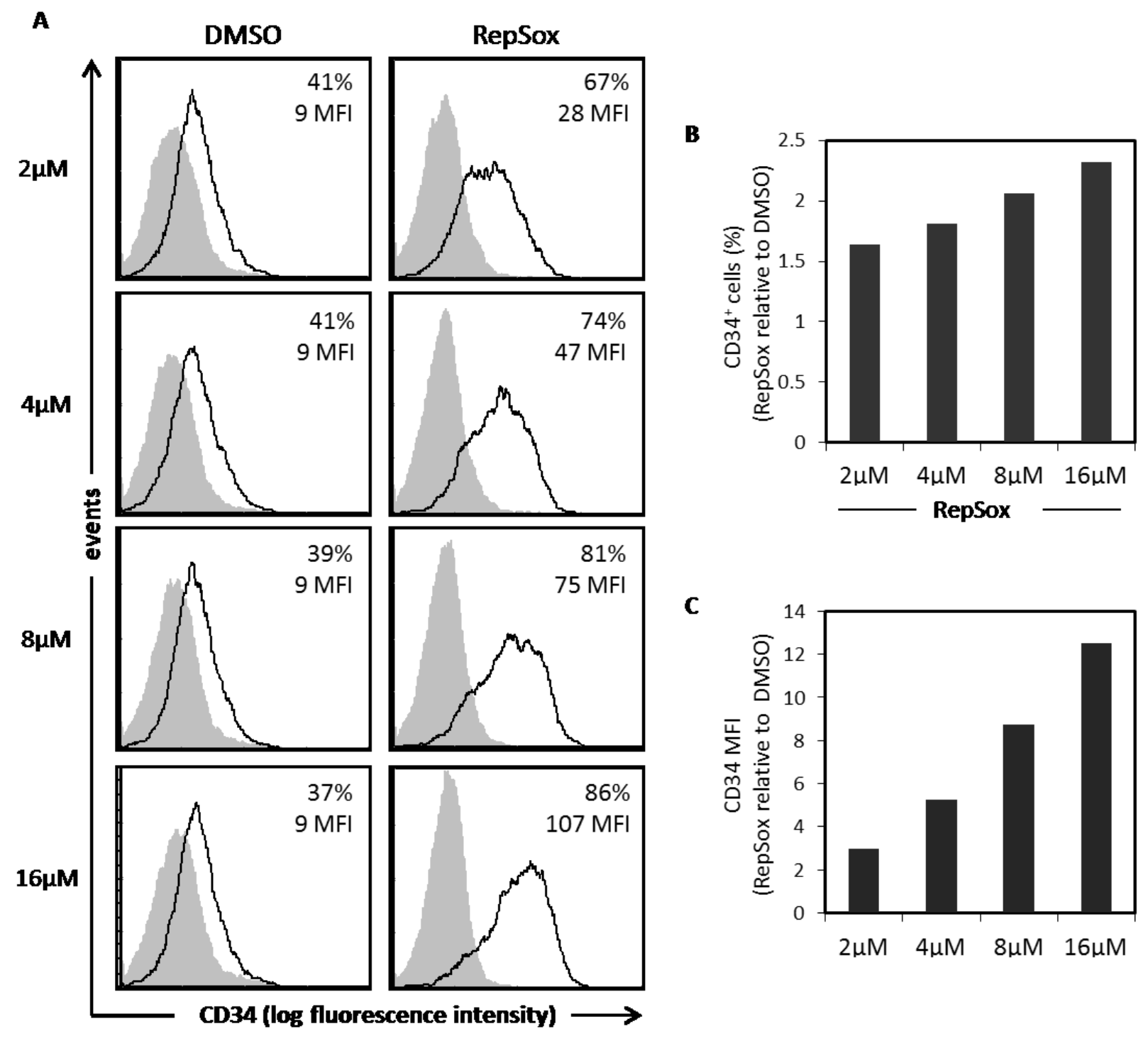

Figure 3. RepSox maintains $\mathrm{CD} 34^{+} \mathrm{AML}$ cells in a concentration-dependent manner. 


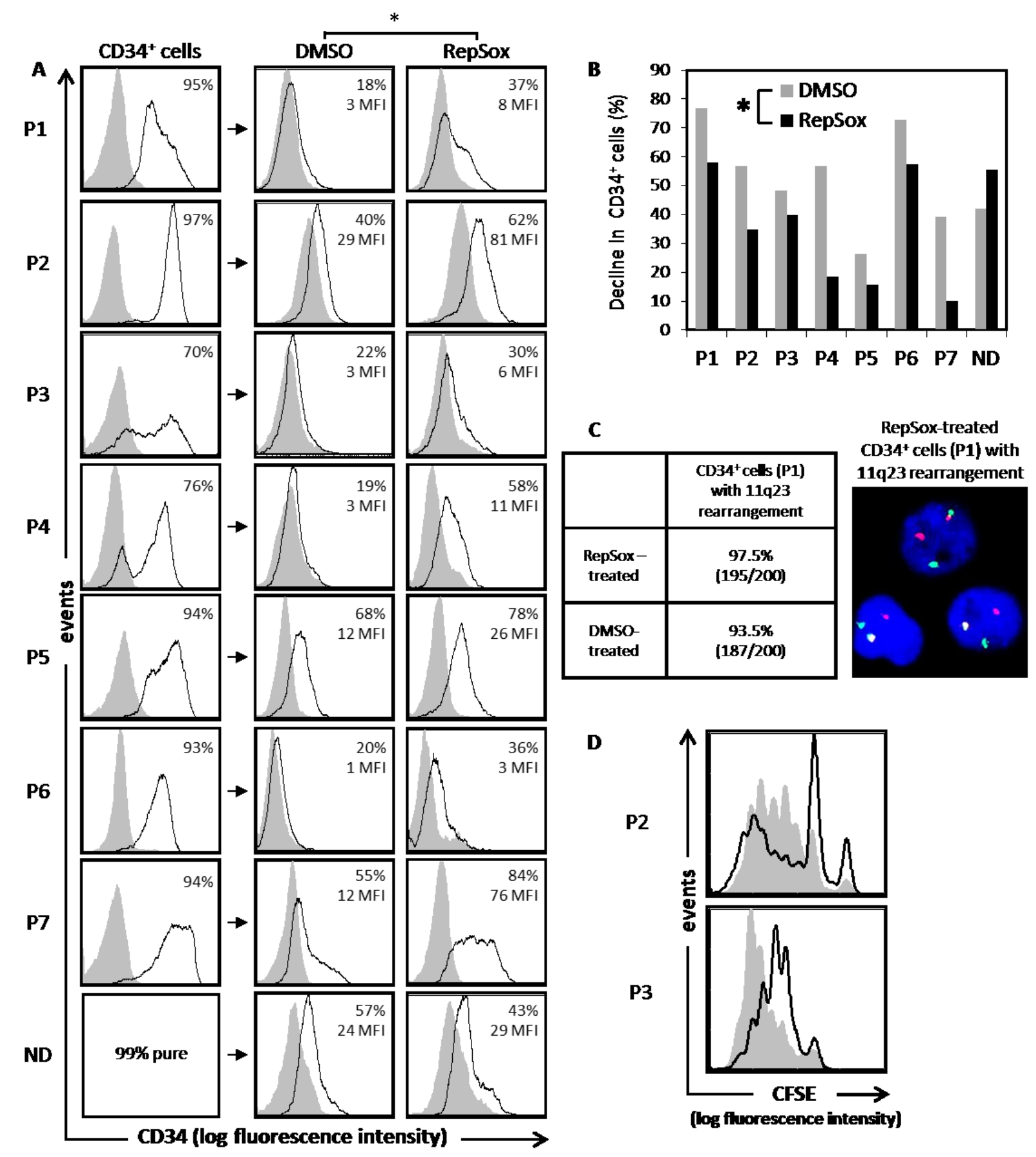

Figure 4. RepSox slows decay of CD34+ $A M L$ and MDS cells. 

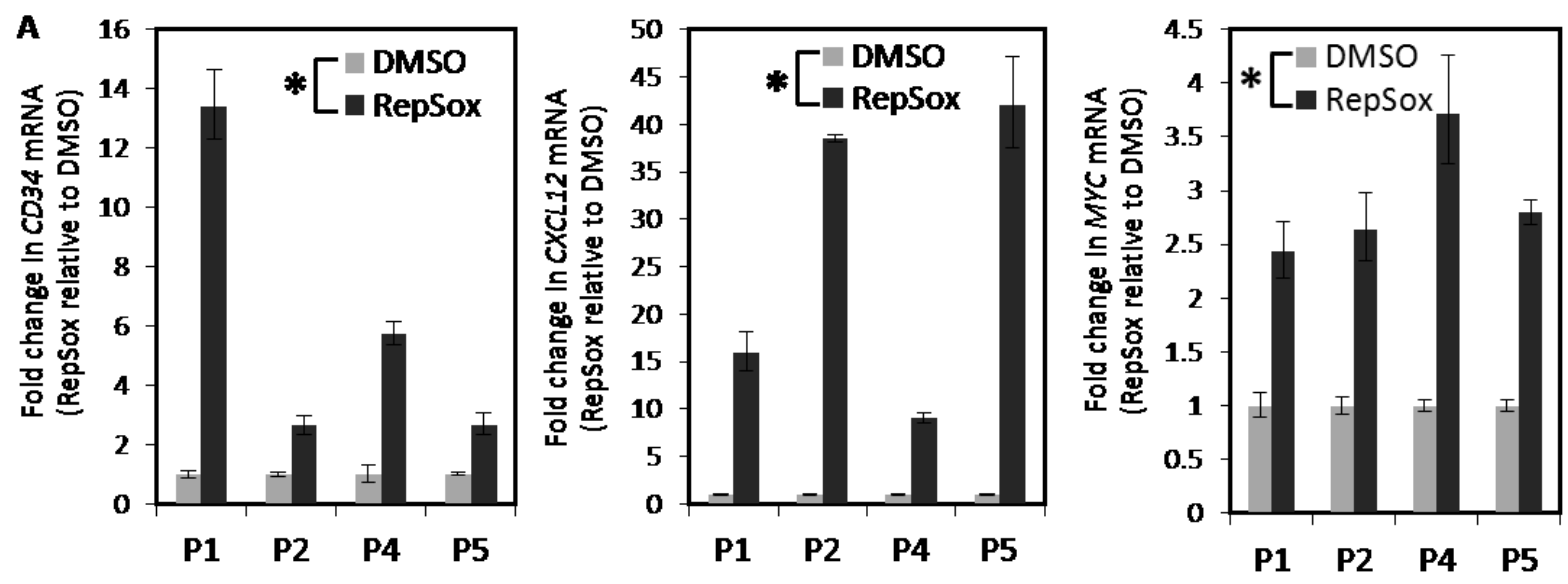

B

P2 CD34+ cells (gated)

P3 CD34+ cells (gated)

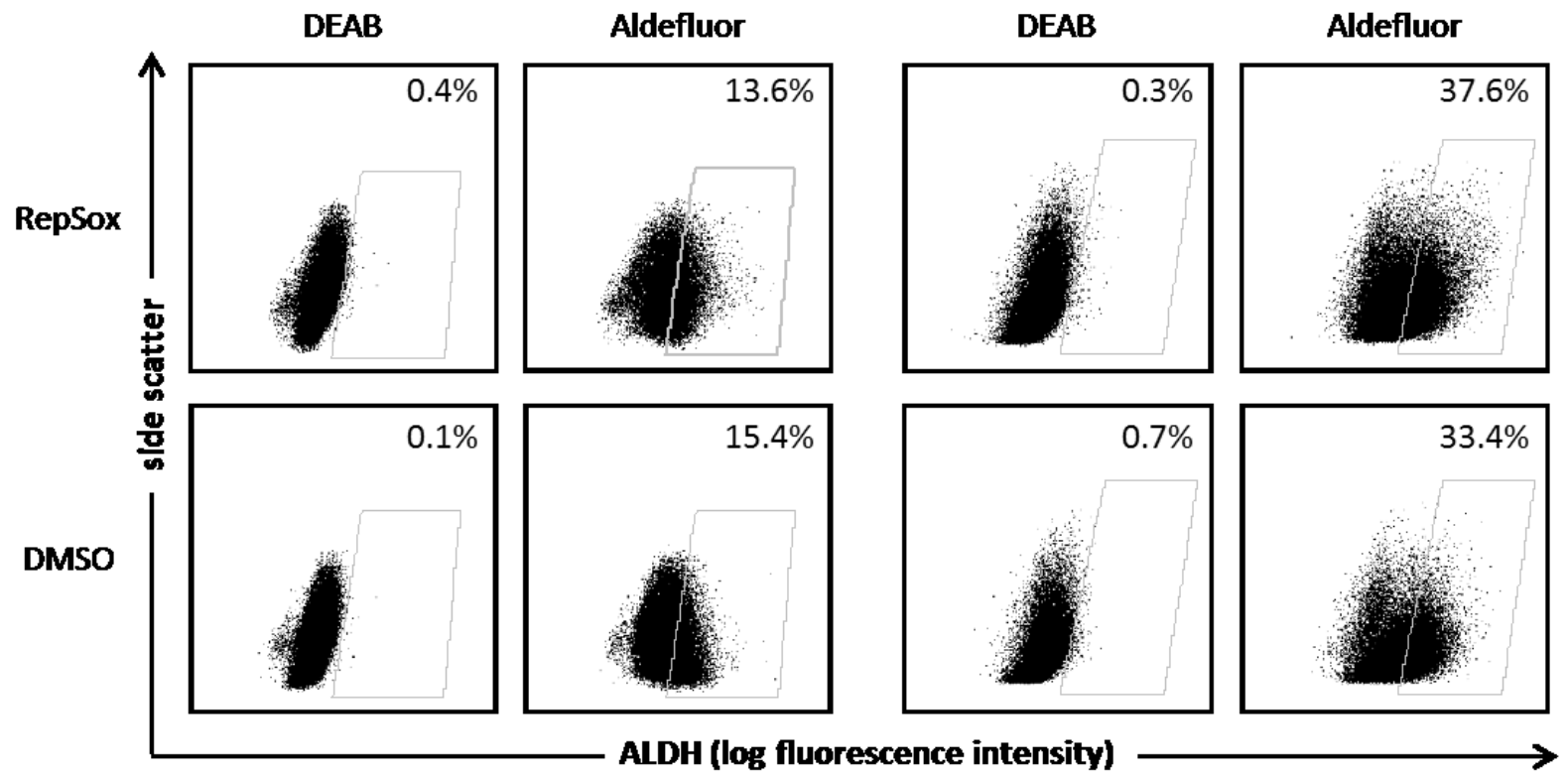

Figure 5. RepSox-treated cells have increased CD34, CXCL12, and MYC mRNA levels and similar ALDH activity compared to DMSO-treated controls. 

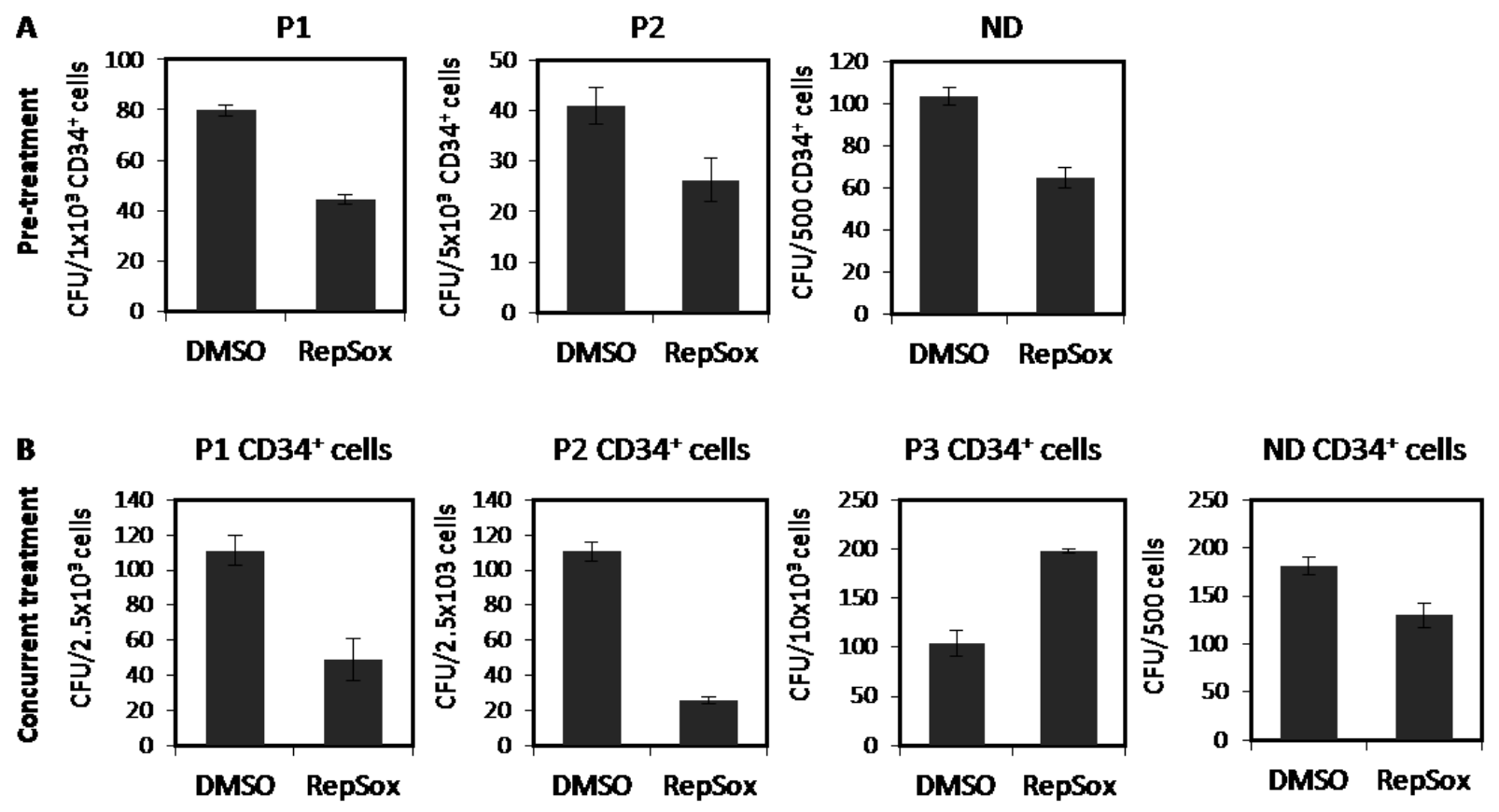

C

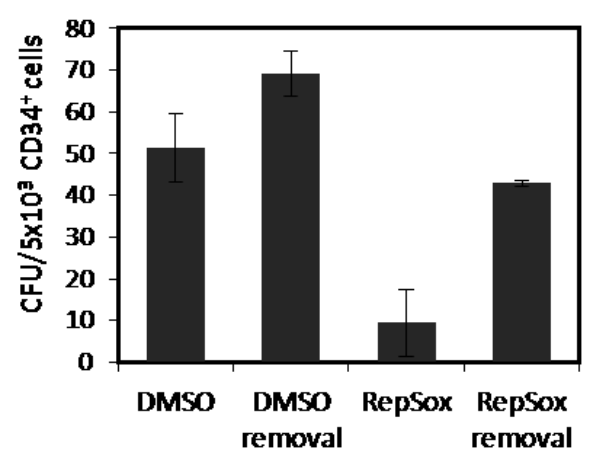

Experimental design

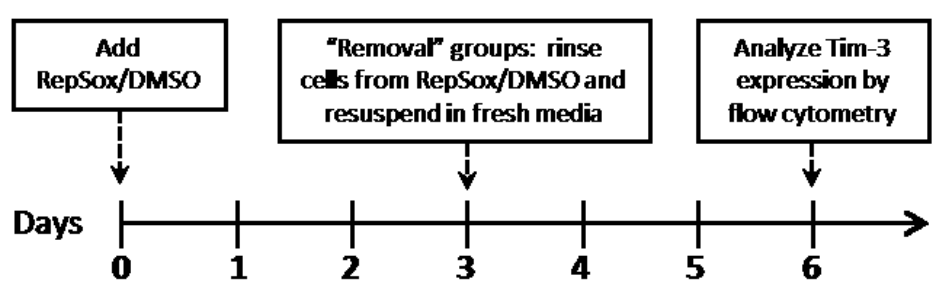

Figure 6. RepSox reversibly suppresses AML colony-forming activity. 

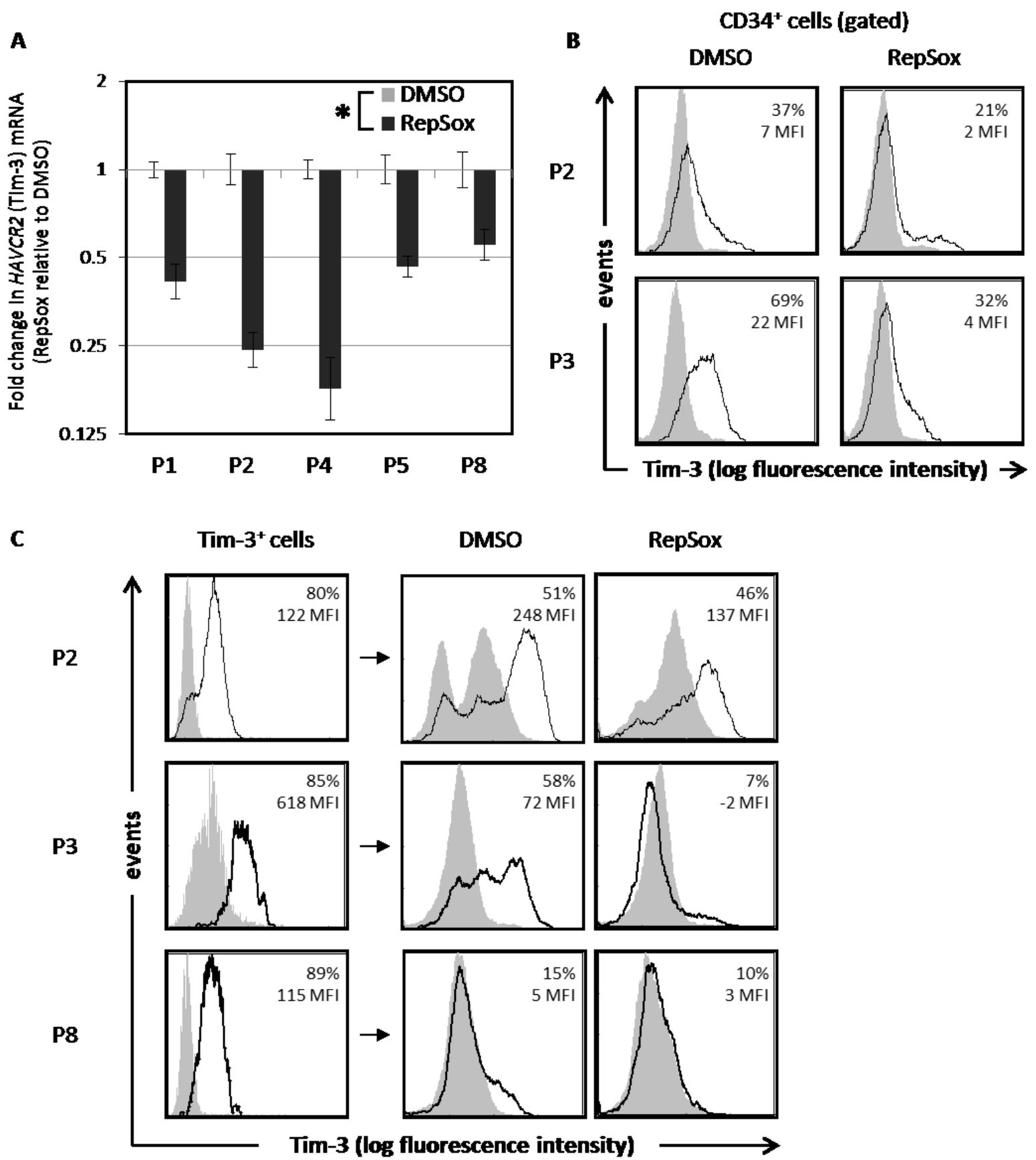

Figure 7. RepSox decreases Tim-3 expression on AML and MDS cells. 


\section{Supplemental Methods}

\section{Phospho-Smad 2/3 intracellular staining}

WBCs were serum-starved for 4 hours in RPMI 1640 medium with serum replacement 1

(Sigma) and subsequently exposed to $10 \mathrm{ng} / \mathrm{mL}$ TGF- $\beta$ (human platelet-derived, R\&D systems) for 30 minutes with $16 \mu \mathrm{M}$ RepSox or DMSO. Following stimulation, cells were fixed in $2 \%$ paraformaldehyde for 10 minutes at room temperature (RT) and chilled on ice for 1 minute. WBCs were permeabilized in cold $90 \%$ methanol for 30 minutes on ice and stored at $-20^{\circ} \mathrm{C}$. Prior to intracellular staining, samples were rinsed with $\mathrm{PBS} / 1 \% \mathrm{BSA}$. Samples were blocked with $1 \mu \mathrm{g}$ human IgG (R\&D systems) for 30 minutes at $4^{\circ} \mathrm{C}$ and incubated with anti-phospho-Smad-2(S465/467)/3(S423/425) or matched rabbit IgG isotype control antibody (Cell Signaling Technologies, Danvers, MA) for 1 hour at RT. Samples were rinsed with PBS/1\% BSA and incubated with AlexaFluor-488 donkey antirabbit secondary antibody (Molecular Probes) for 1 hour at RT. Cells were rinsed and analyzed by flow cytometry.

\section{Western blot analysis}

Total cellular protein $(25 \mu \mathrm{g} / \mathrm{sample})$ was separated over $4-20 \%$ gradient polyacrylamide gels (Bio-Rad, Hercules, CA). Proteins were transferred to nitrocellulose membranes (Whatman, Dassel, Germany) and blocked for 60 minutes in TBS/0.1\% Tween-20 (TBST) containing 5\% non-fat dry milk. Blots were incubated with a 1:1,000 dilution of antiphospho-Smad2 (Ser465/467) antibody (Cell Signaling) in PBS $/ 5 \%$ BSA overnight at $4^{\circ} \mathrm{C}$ with gentle rocking. Blots were rinsed 3 times with TBS-T for 5 minutes and incubated with a 1:5000 dilution of HRP-linked anti-rabbit IgG (Cell Signaling). Blots were rinsed with TBS-T 3 times for 5 minutes, incubated with Immobilon chemiluminescent HRP 
substrate (Millipore, Billerica, MA), and developed. MagicMark molecular weight standard (Bio-Rad) was used to estimate protein size. GAPDH served as a loading control.

\section{Growing and imaging 3-D leukemia spheroids}

Leukemia cells and BM osteoblasts were labeled with CellTracker green CMFDA (Molecular Probes) and CellTrace Far Red DDAO-SE (Molecular Probes) fluorescent probes and co-cultured on ultra-low attachment plates (Corning, Corning, NY). For 3-D

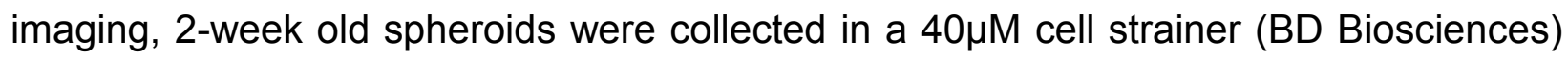
and imaged by 2-photon microscopy using a 60X water-dipping objective. Images were deconvoluted using AutoQuant software (Media Cybernetics, Inc., Warrendale, PA) and imported into NIS-Elements AR imaging software (Nikon) for 3-D viewing.

\section{WBC expansion}

WBCs were co-cultured with BM stromal cells in RPMI 1640 medium supplemented with serum replacement 1 (Sigma) for 10 days. To expand cells, recombinant human IL-3 (20 $\mathrm{ng} / \mathrm{mL})$, G-CSF $(20 \mathrm{ng} / \mathrm{mL})$, and SCF $(100 \mathrm{ng} / \mathrm{mL})$ were added at the initiation of culture and 5 days later.

\section{5-ethynyl-2'-deoxyuridine (EdU) staining}

To measure cell proliferation, the Click-iT EdU Alexa Fluor 488 Flow Cytometry Assay Kit (Molecular Probes, Eugene, OR, USA) was used according to the manufacturer's instructions. WBCs were co-cultured with BM stromal cells for 3 days, $1 \mu \mathrm{M}$ EdU (a nucleoside analog to thymidine) was added, and cells were cultured for another 3 days. Cells were fixed with $4 \%$ paraformaldehyde and permeablized with saponin. EdU incorporation into DNA was detected by a copper catalyzed covalent reaction. 
Surface immunostaining and flow cytometric analysis for RepSox differentiation experiment

Samples were stained according to standard whole-blood lysis procedure using the Becton Dickinson lyse-wash method. Monoclonal antibodies directed against the following human antigens were used: CD19-PE (4G7), CD33-PerCP-Cy5.5 (P67.7), CD3-APC (SK7), CD34-PE-Cy7 (8G12), CD45-APC-Cy7, CD8-FITC (SK1), CD4-PE (DK3), CD3-PerCP-Cy5.5 (SK7), CD14-APC (MфPg), and CD56-PE-Cy7 (NCAM16.2) (Becton Dickinson). The following 3-tube panel was prepared for each sample: isotype controls and CD45 (tube 1), CD19/CD33/CD3/CD34/CD45 (tube 2), and CD8/CD4/CD3/CD14/CD56/CD45 (tube 3). Samples were analyzed by FACS using a Becton Dickinson FACSCanto II. A minimum of 30,000 events were acquired and data were analyzed using FCSExpress software (De Novo Software). Initial gating was based on forward scatter versus side scatter dot plots to exclude dead cells and debris. Thresholds for positivity were set to exclude $>99 \%$ of isotype control antibody-stained cells. Positive events were back-gated to ensure that they constituted a discrete population on CD45 versus side scatter dot plots to confirm specificity of antigen binding. 


\section{Supplemental Table and Figure Legends}

Table S1. Characteristics of leukapheresis specimens. AML specimens were evaluated for $\mathrm{t}(8 ; 21), \mathrm{t}(15 ; 17)$, inv(16), and 11q23 alterations, while the MDS/MPS specimen was tested for $4 q 12,5 q 32,8 p 12$, and $t(9 ; 22)$ chromosomal abnormalities by fluorescence in situ hybridization (FISH). All samples were screened for FLT3-ITD, NPM1, and CEBPA mutations by polymerase chain reaction (PCR).

a Stage of disease at the time of leukapheresis collection.

${ }^{\mathrm{b}}$ Measurements obtained prior to leukapheresis.

c Information not available.

Abbreviations: WBC, white blood cell; MLL, mixed-lineage leukemia; ELL, elongation factor RNA polymerase II; MDS, myelodysplastic syndrome; MPS, myeloproliferative syndrome; del, deletion; inv, inversion; FLT3, fms-related tyrosine kinase 3; ITD, internal tandem duplication; NPM1, nucleophosmin; and CEBPA, CCAAT/enhancer binding protein $\alpha$.

Figure S1. Purity of CD $34^{+}$and CD34- fractions prior to measuring colony-forming activity. AML/MDS leukapheresis specimens were immunomagnetically sorted into $\mathrm{CD} 4^{+}$and CD34- subsets. Purity of fractions was evaluated by flow cytometry after CD34 surface staining (open histograms). Solid histograms display isotype controls.

Figure S2. CD34 ${ }^{+} \mathrm{AML}$ and MDS cells generally display greater ALDH activity than CD34- cells. ALDH activity of $\mathrm{CD} 34^{+}$and $\mathrm{CD} 34^{-}$cells (selected by gating against isotype controls) measured by flow cytometry. $\mathrm{ALDH}^{+}$regions were drawn to exclude $>99 \%$ of DEAB-treated (negative control) cells. 
Figure S3. RepSox does not induce CD34 expression on CD34- AML cells. Purified CD34- AML cells were cultured with $16 \mu \mathrm{M}$ RepSox or DMSO (on stroma, $10 \%$ FBS, $5 \%$ $\mathrm{O}_{2}$ ). After 6 days, CD34 surface expression (open histograms) was evaluated by flow cytometry. Solid histograms display isotype controls.

Figure S4. RepSox-treated CD $34^{+}$cells expand as effectively as DMSO controls upon cytokine stimulation. AML cells $(\mathrm{P} 1)$ were cultured with $16 \mu \mathrm{M}$ RepSox or DMSO (on stroma, serum-free, $5 \% \mathrm{O}_{2}$ ). Recombinant human IL-3 $(20 \mathrm{ng} / \mathrm{mL})$, G-CSF (20 $\mathrm{ng} / \mathrm{mL})$, and SCF (100 ng/mL) were added at days 0 and 5. After 10 days, (A) WBCs were counted (bar graphs display mean counts \pm SE from 2 independent experiments) and (B) CD34 expression (open histograms) was evaluated by flow cytometry. Solid histograms display isotype controls.

Figure S5. Generation of 3-D spheroids containing leukemic cells and osteoblasts. Primary acute lymphoblastic leukemia (ALL) cells (green) and BM osteoblasts (red) were labeled with fluorescent probes and co-cultured on low attachment plates to generate 3D spheroids. Spheroids were imaged by 2-photon microscopy (600X magnification) after two weeks.

Figure S6. RepSox inhibits TGF- $\beta$-induced phosphorylation of Smad2/3. AML cells (P1) were serum-starved for 4 hours and cultured (A) \pm TGF- $\beta$ (10 $\mathrm{ng} / \mathrm{mL})$ for 30 minutes and (B) with $16 \mu \mathrm{M}$ RepSox or DMSO (vehicle control) for 30 minutes prior to TGF- $\beta$ (10 $\mathrm{ng} / \mathrm{mL}$ ) exposure for 30 minutes. Phosphorylated Smad-2/3 expression was evaluated by flow cytometry. (C) Representative immunoblot of AML cells (P6) serum-starved for 4 
hours then cultured with $16 \mu \mathrm{M}$ RepSox or DMSO for 30 minutes prior to TGF- $\beta(10 \mathrm{ng} / \mathrm{mL})$ exposure for 30 minutes. D, DMSO; RS, RepSox.

Figure S7. Unlike RepSox, additional TGF- $\beta$ inhibitors do not slow decay of CD34 ${ }^{+}$ AML cells. (A) CD34 ${ }^{+}$AML cells (P1) were cultured with TGF- $\beta$ inhibitors (LY364947, GW788388, SB431542) or DMSO (vehicle control) for 6 days (on stroma, 10\% FBS, 5\%

$\mathrm{O}_{2}$ ). CD34 surface expression (open histograms) was evaluated by flow cytometry. Solid histograms display isotype controls. (B) Chemical and physical structures of TGF- $\beta$ inhibitors evaluated. (C) qRT-PCR analysis of MYC and CXCL12 mRNA levels following 48-hour exposure of AML cells (P6 and P8) to various TGF- $\beta$ inhibitors.

Figure S8. RepSox-treated CD34+ cells proliferate more slowly than DMSO controls. $\mathrm{CD}_{3} 4^{+} \mathrm{AML}$ cells were cultured (on stroma, $10 \% \mathrm{FBS}, 5 \% \mathrm{O}_{2}$ ) with $16 \mu \mathrm{M}$ RepSox or DMSO with and without EdU (added after 3 days). After a total of 6 days in culture, EdU incorporation (open histograms) by $\mathrm{CD} 34^{+}$cells (selected by gating) was evaluated by flow cytometry. Solid histograms display cells cultured without EdU.

Figure S9. Gating strategy used to select CD34 ${ }^{+}$cells. To select $\mathrm{CD} 34^{+}$cells, regions were drawn on side scatter versus CD34 fluorescence dot plots to include anti-CD34 antibody-stained cells, but exclude $>98 \%$ of isotype control antibody-stained cells. Regions were converted to gates for subsequent analyses of CD $34^{+}$cells.

Figure S10. Increased proportion of $\mathrm{Tim}^{-3^{+}}$cells following removal of RepSox. Flow cytometric analysis of Tim-3 surface expression (open histograms) on MDS (P3) cells cultured with $16 \mu \mathrm{M}$ RepSox or DMSO (on stroma, 10\% FBS, $5 \% \mathrm{O}_{2}$ ): 
continuously for 6 days and (B) intermittently for 3 days prior to rinsing and culturing in fresh media for 3 additional days. Solid histograms display isotype controls.

Figure S11. TGF- $\beta$ inhibitors RepSox and SB431542 reduce Tim-3 expression on AML and MDS cells. FACS-purified Tim- $3^{+}$AML/MDS cells (open histograms) were cultured with $16 \mu \mathrm{M}$ RepSox, $2 \mu \mathrm{M}$ SB431542, or DMSO (vehicle control) for 6 days (on stroma, $10 \% \mathrm{FBS}, 5 \% \mathrm{O}_{2}$ ). Solid histograms display isotype controls. Surface expression of Tim-3 and CD3 (T cell marker) was evaluated by flow cytometry.

Figure S12. RepSox did not drastically alter CD47, HLA-A/B/C, or MUC1 expression on CD34 ${ }^{+}$AML and MDS cells. AML (P2) and MDS (P3) cells were cultured with $16 \mu M$ RepSox or DMSO for 6 days (on stroma, $10 \%$ FBS, $5 \% \mathrm{O}_{2}$ ). Surface expression of Tim3, CD47, HLA-A/B/C, and MUC1 (open histograms) on CD34+ cells (selected by gating) was evaluated by flow cytometry. Solid histograms display isotype controls. 
Supplemental Table and Figures

\begin{tabular}{|c|c|c|c|c|c|c|c|}
\hline $\begin{array}{l}\text { Patient } \\
\text { (P) }\end{array}$ & $\begin{array}{l}\text { Age, } \\
\text { Gender }\end{array}$ & Diagnosis & Status ${ }^{\mathbf{a}}$ & $\begin{array}{l}\text { Molecular and } \\
\text { cytogenetic } \\
\text { abnormalities }\end{array}$ & $\begin{array}{c}\text { Peripheral WBC } \\
\text { count }^{\mathbf{b}} \\
\left(1 \times 10^{3} \text { cells/} / \mu l\right)\end{array}$ & $\begin{array}{c}\text { Blasts }(\boldsymbol{\%}) \text { in } \\
\text { peripheral blood, } \\
\text { bone marrowr }\end{array}$ & 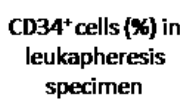 \\
\hline P1 & $24, M$ & $\begin{array}{l}\text { Acute myeloid } \\
\text { leukemia with } \\
11 \mathrm{q} 23 \text { (MW) } \\
\text { abnormalities }\end{array}$ & Diagnosis & $\begin{array}{c}\text { t(11;19)(q23;p13.1), } \\
\text { MU-EU }\end{array}$ & 52 & $55 \%, 76 \%$ & $57 \%$ \\
\hline $\mathbf{P 2}$ & $48, M$ & $\begin{array}{c}\text { Acute } \\
\text { myelomonocytic } \\
\text { leukemia(M4) }\end{array}$ & Relapse & none detected by FISH & 102 & $17 \%, 40 \%$ & $32 \%$ \\
\hline P3 & $66, M$ & $\begin{array}{c}\text { MDS/MPS, } \\
\text { unclassifiable in } \\
\text { accelerated phase }\end{array}$ & Diagnosis & $\begin{array}{l}t(5 ; 13)(q 13 ; q 12) \\
\text { del(6)(q21) }\end{array}$ & 234 & $12 \%, 13 \%$ & $54 \%$ \\
\hline P4 & $45, F$ & $\begin{array}{c}\text { Acute } \\
\text { monoblastic/ } \\
\text { monocytic } \\
\text { leukemia(M5) }\end{array}$ & Diagnosis & $\begin{array}{c}\text { Inv(16), } \\
\text { FLT3 D835 variant+ }\end{array}$ & 133 & $23 \%, 69 \%$ & $21 \%$ \\
\hline P5 & - & $\begin{array}{l}\text { Acute myeloid } \\
\text { leukemia }\end{array}$ & - & $\begin{array}{l}\text { t(8;21), } \\
\text { AML-ETO }\end{array}$ & - & - & $83 \%$ \\
\hline P6 & $59, F$ & $\begin{array}{c}\text { Acute } \\
\text { myelomonocytic } \\
\text { leukemia(M4) }\end{array}$ & Diagnosis & $\begin{array}{l}\text { FLT3-ITD+, } \\
\text { NPM1+ }\end{array}$ & 45 & $59 \%, 84 \%$ & $48 \%$ \\
\hline P7 & - & $\begin{array}{l}\text { Acute myeloid } \\
\text { leukemia }\end{array}$ & - & none detected by FISH & - & - & 98\% \\
\hline P8 & $71, F$ & $\begin{array}{l}\text { Acute myeloid } \\
\text { leukemia with } \\
\text { monocytic } \\
\text { differentiation } \\
\text { (M5) }\end{array}$ & Diagnosis & $\begin{array}{l}\text { FLT3-ITD+, } \\
\text { NPM1+ }\end{array}$ & 119 & $37 \%, 90 \%$ & $4 \%$ \\
\hline
\end{tabular}

Table S1. Characteristics of leukapheresis specimens. 


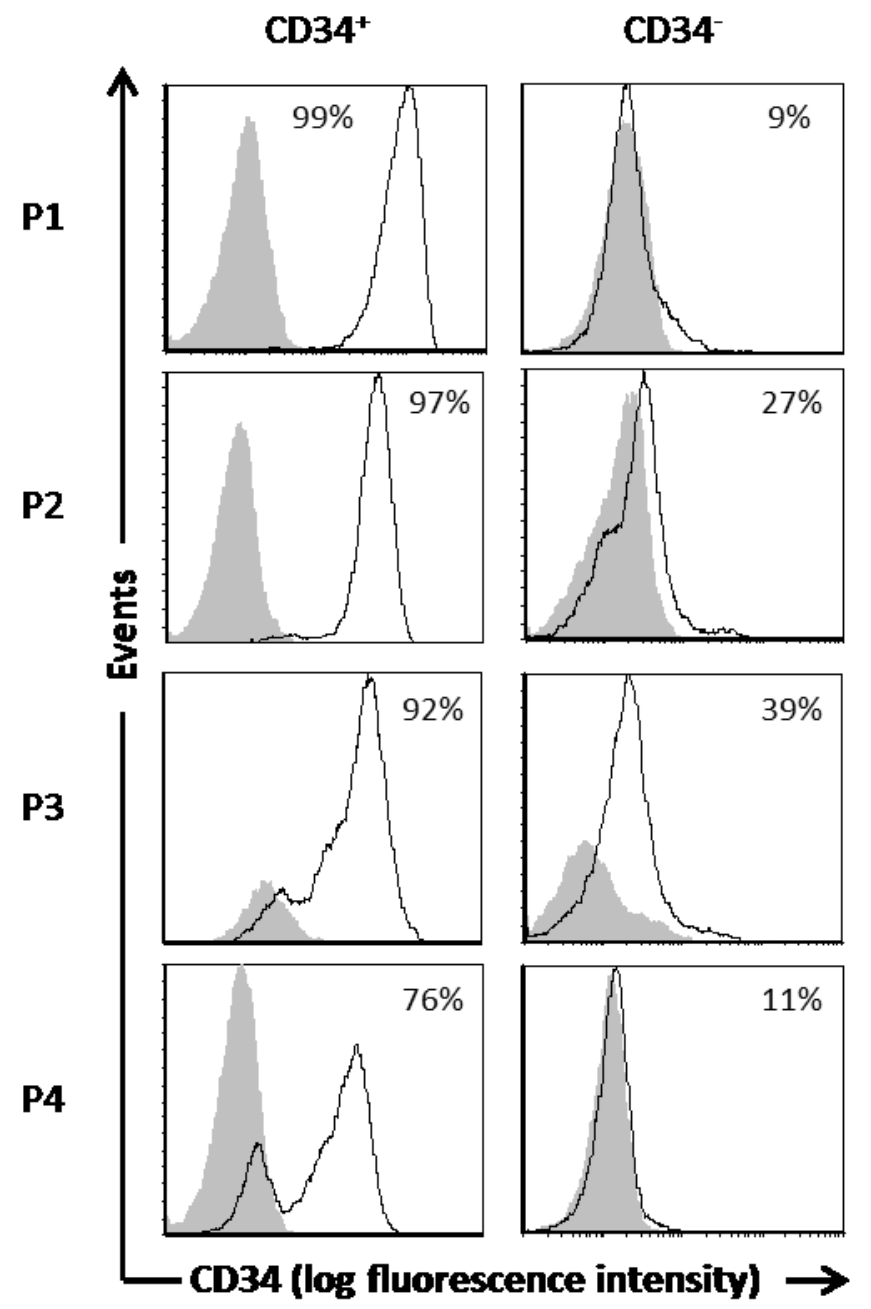

Figure S1. Purity of CD34 ${ }^{+}$and CD34- fractions prior to measuring colony-forming activity. 


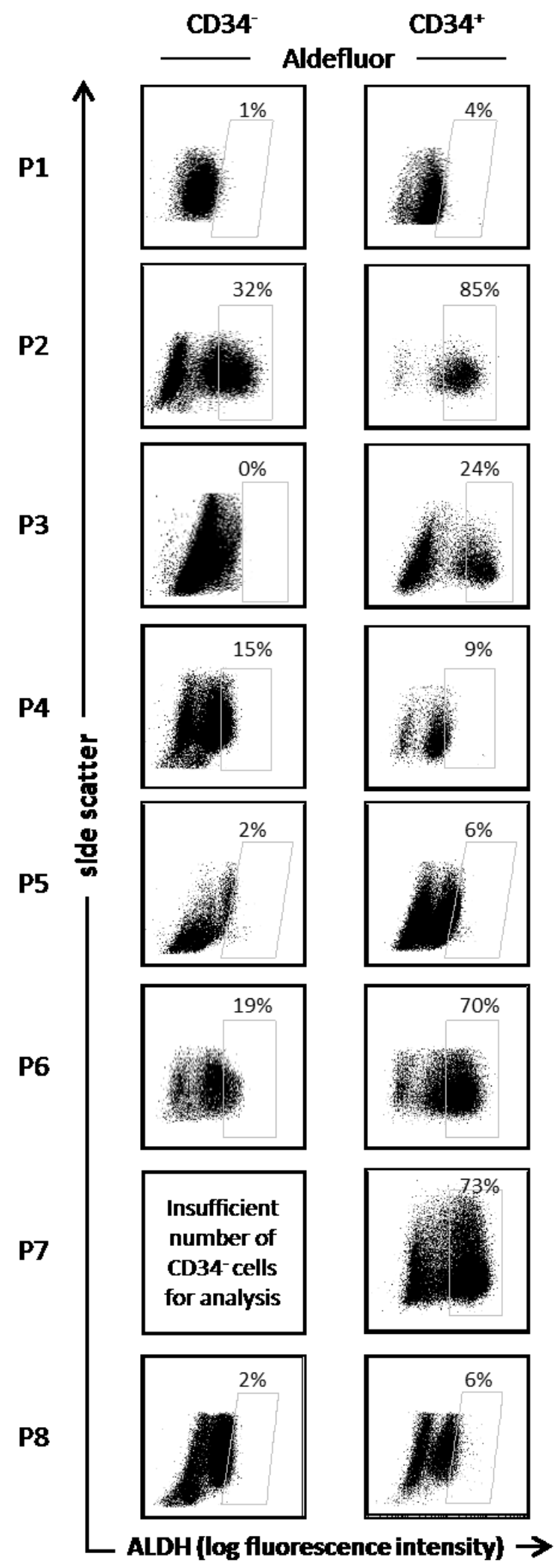

Figure S2. CD34 ${ }^{+} \mathrm{AML}$ and MDS cells generally display greater ALDH activity than CD34- cells. 


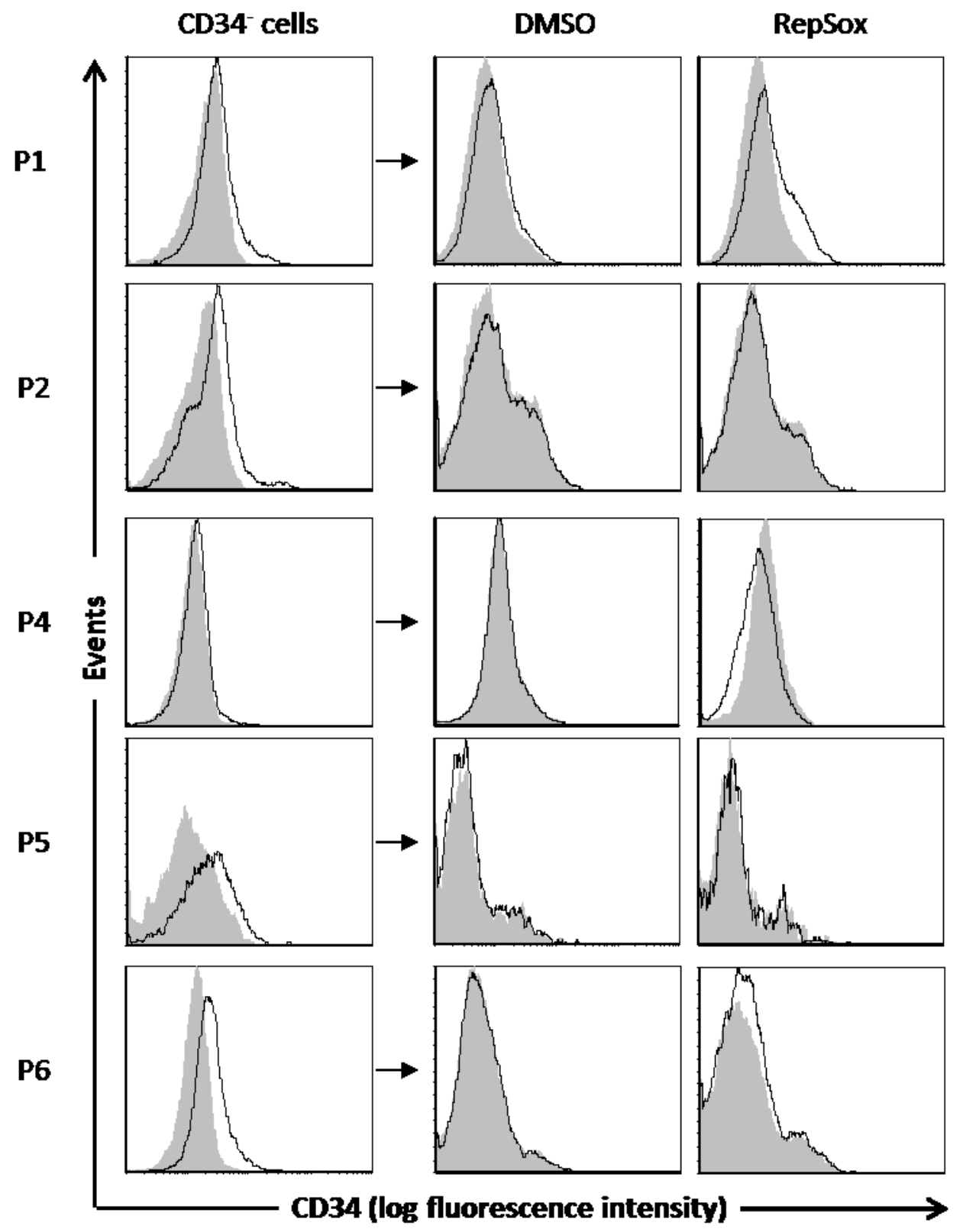

Figure S3. RepSox does not induce CD34 expression on CD34- AML cells. 
A

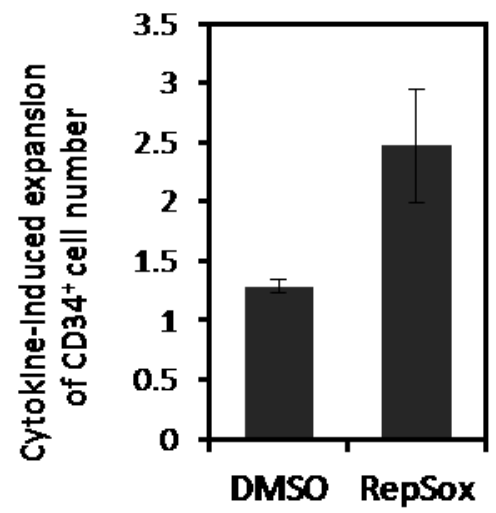

B

- cytokines

+ cytokines

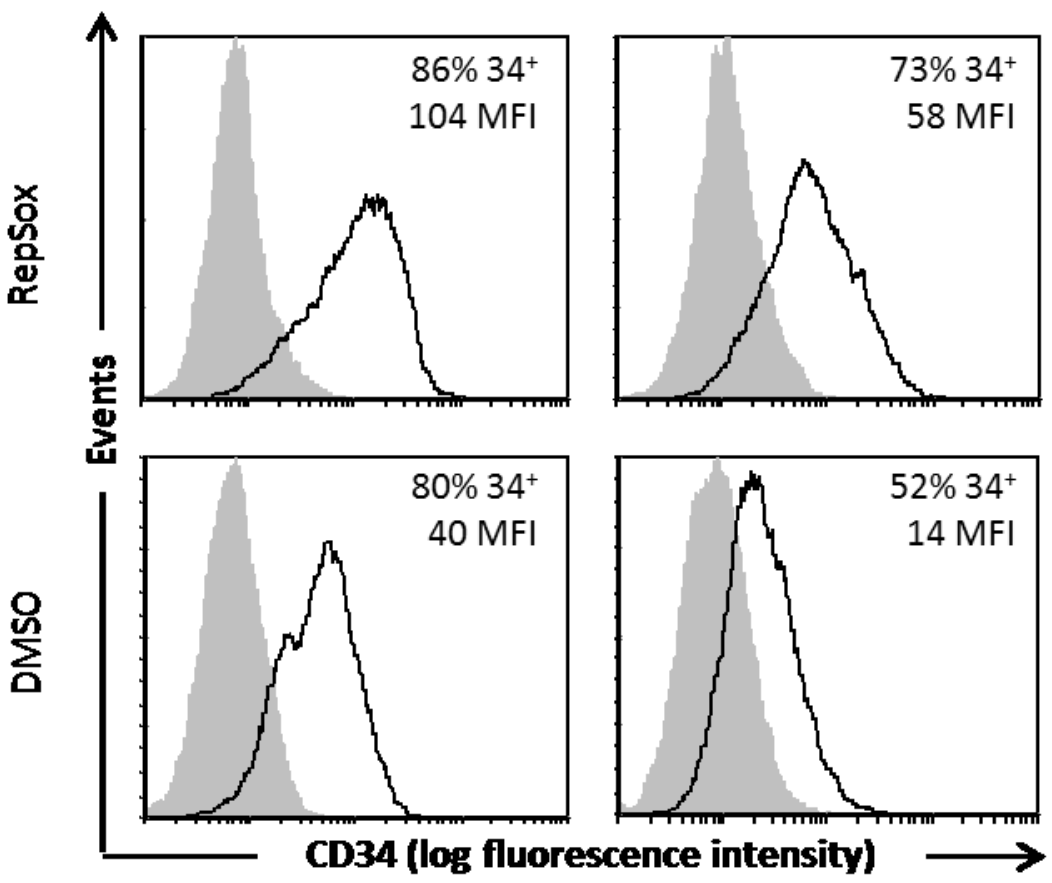

Figure S4. RepSox-treated CD34+ cells expand as effectively as DMSO controls upon cytokine stimulation. 


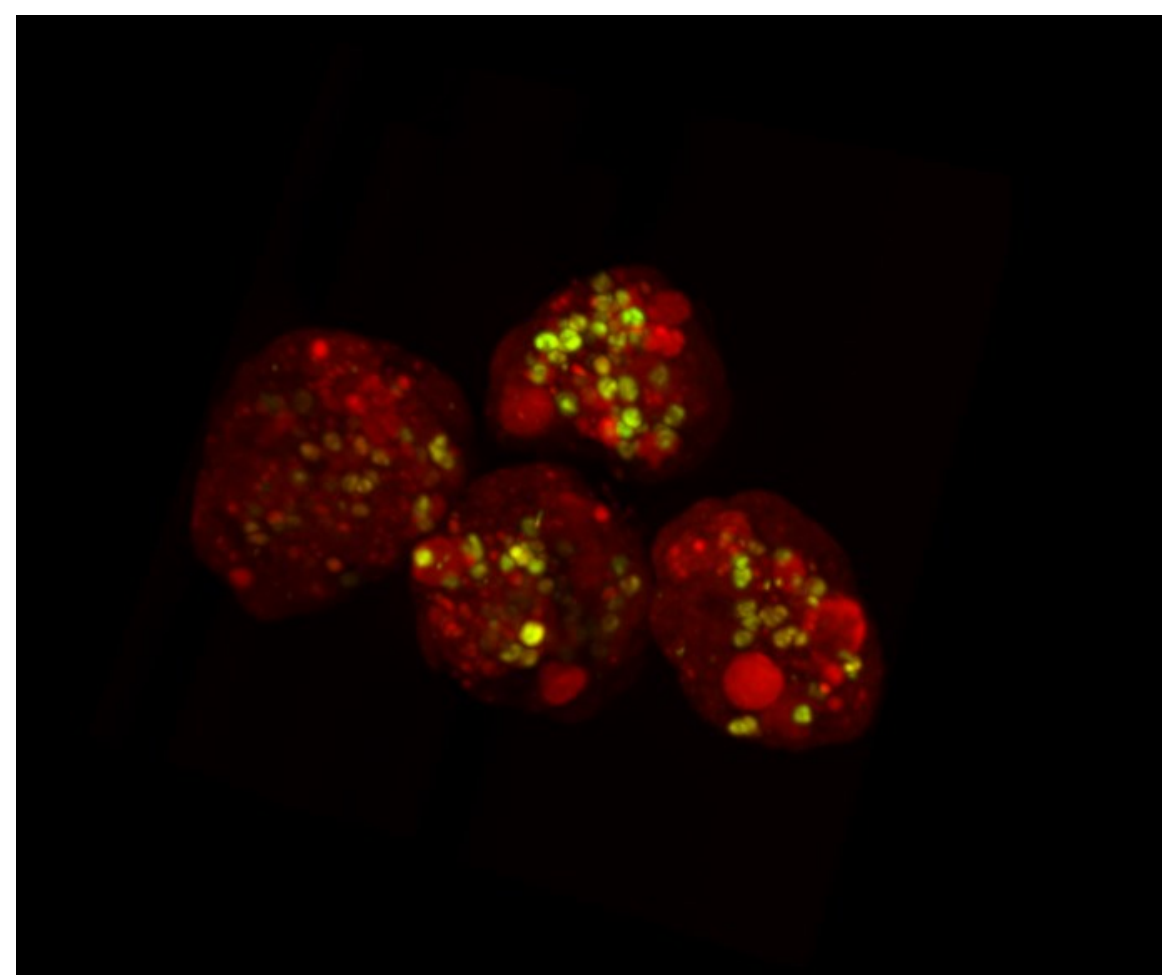

Figure S5. Generation of 3-D spheroids containing leukemic cells and osteoblasts. 

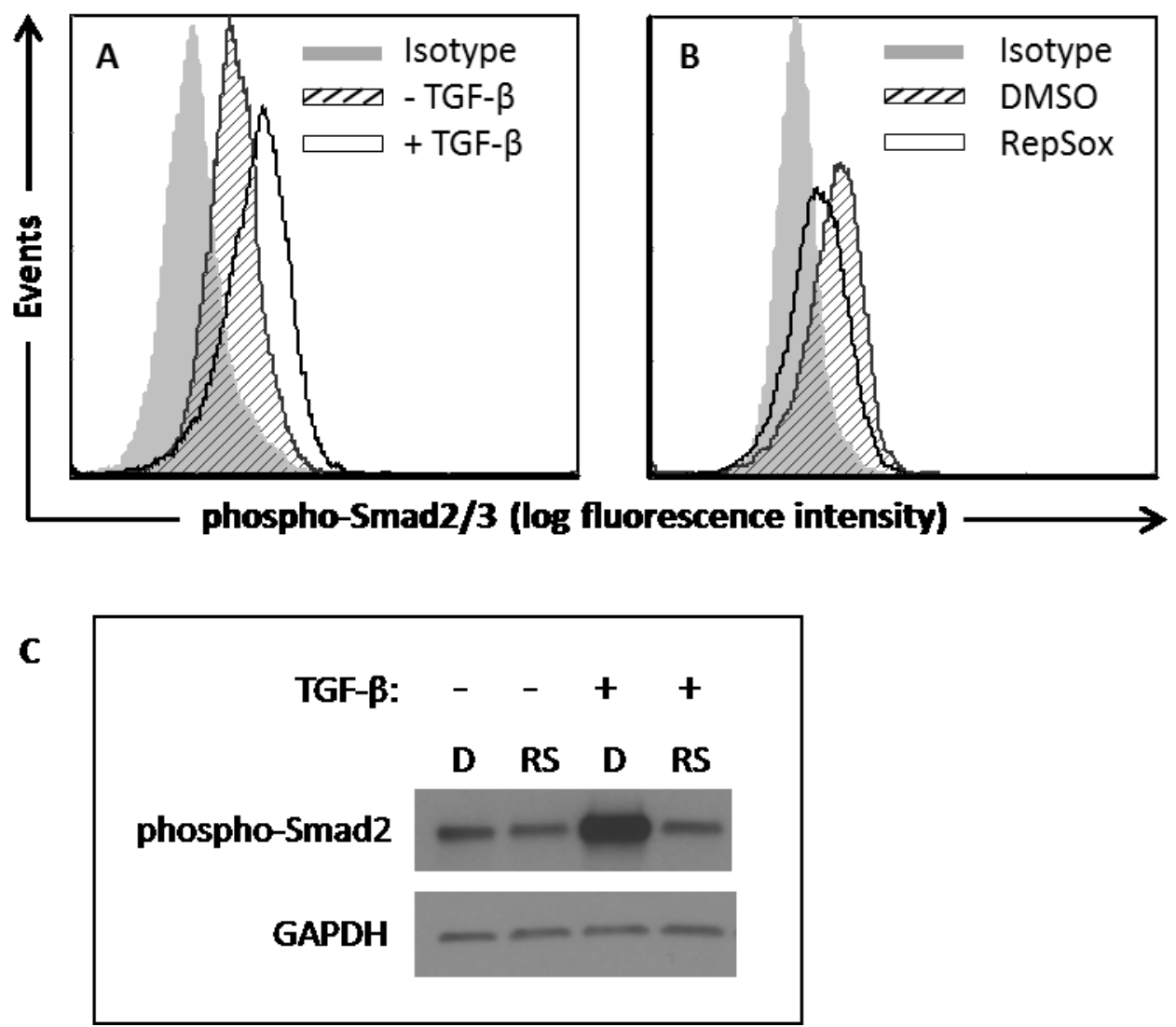

Figure S6. RepSox inhibits TGF- $\beta$-induced phosphorylation of Smad2/3. 
A

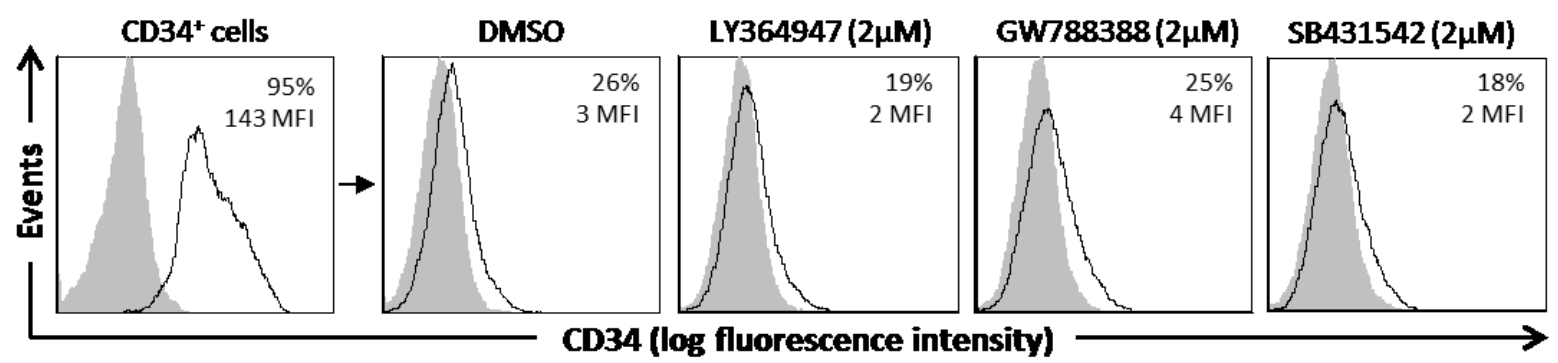

B RepSox<smiles>Cc1cccc(-c2n[nH]cc2-c2ccc3ncccc3n2)n1</smiles>

$\mathrm{C}_{17} \mathrm{H}_{13} \mathbf{N}_{5}$
LY364947

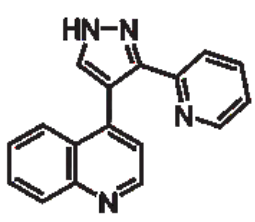

$\mathrm{C}_{17} \mathrm{H}_{12} \mathrm{~N}_{4}$
GW788388

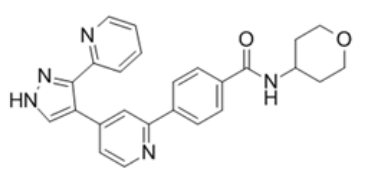

$\mathrm{C}_{25} \mathrm{H}_{23} \mathrm{~N}_{5} \mathrm{O}_{2}$

SB431542

C
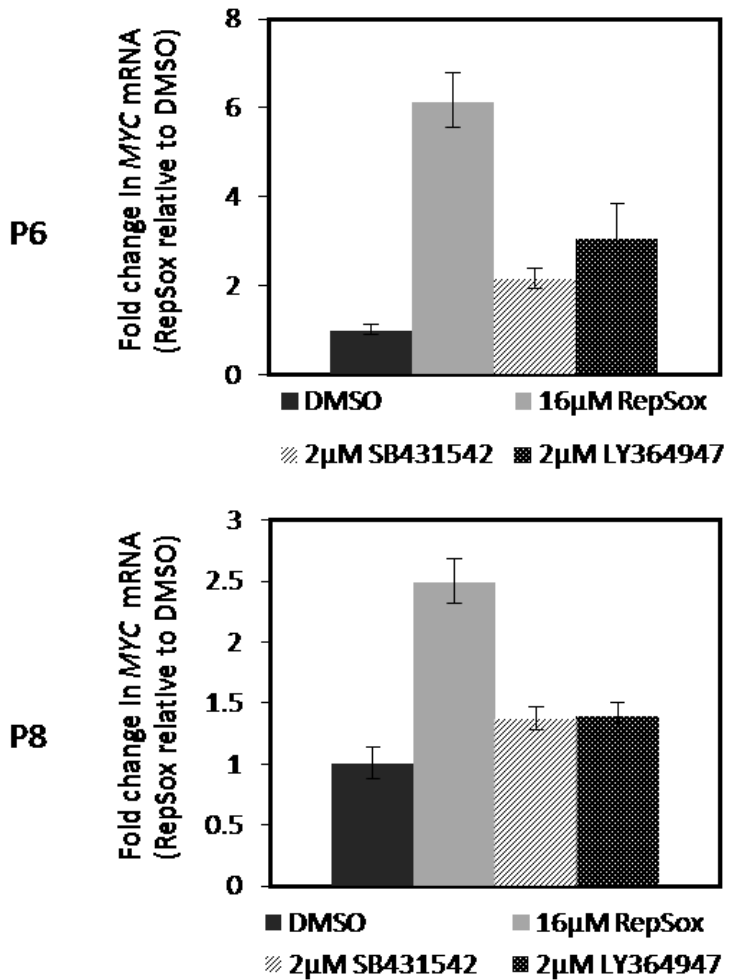
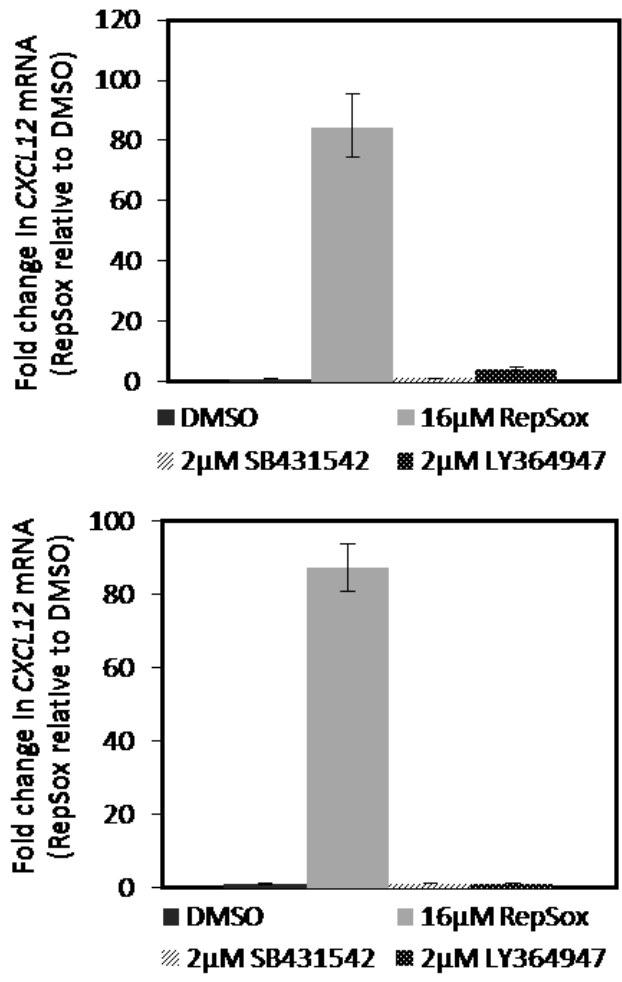

Figure S7. Unlike RepSox, additional TGF- $\beta$ inhibitors do not slow decay of CD34 ${ }^{+}$ AML cells. 


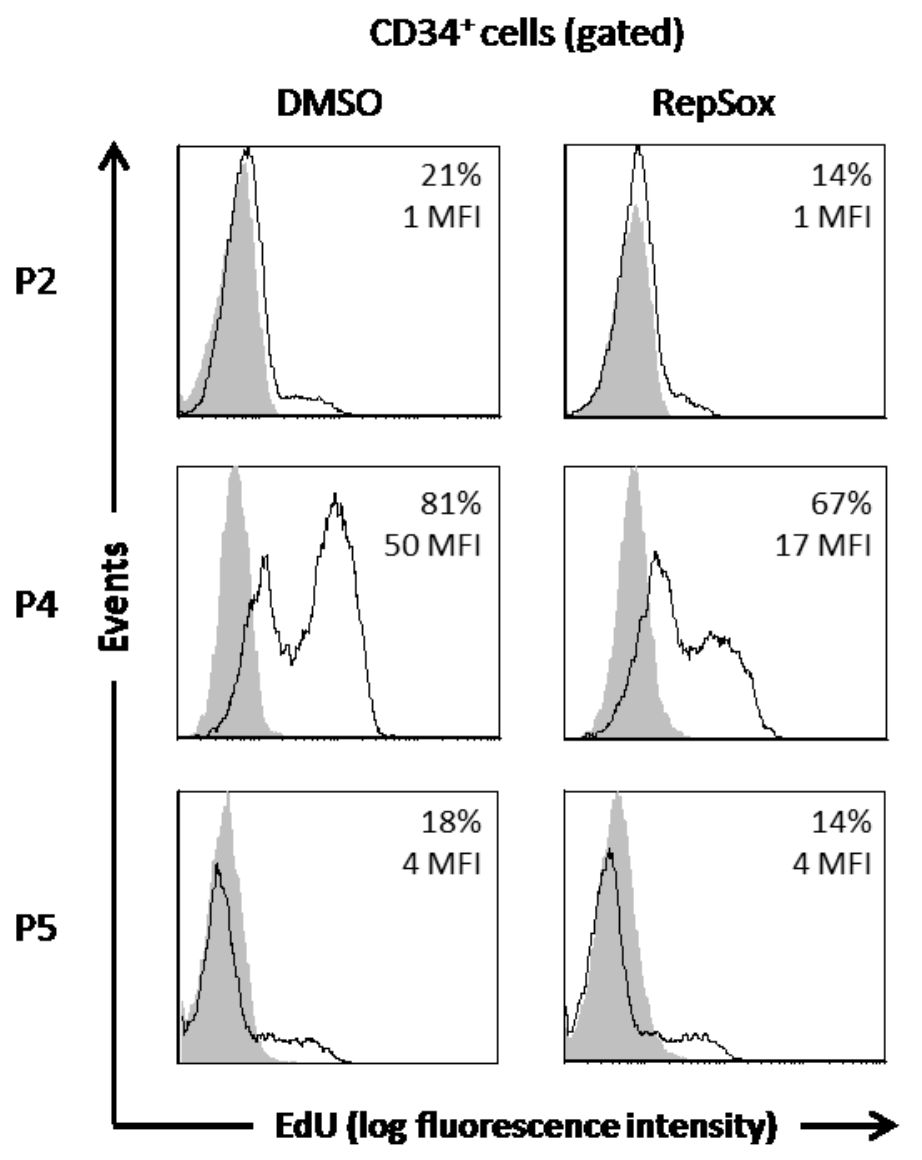

Figure S8. RepSox-treated CD34+ cells proliferate more slowly than DMSO controls. 


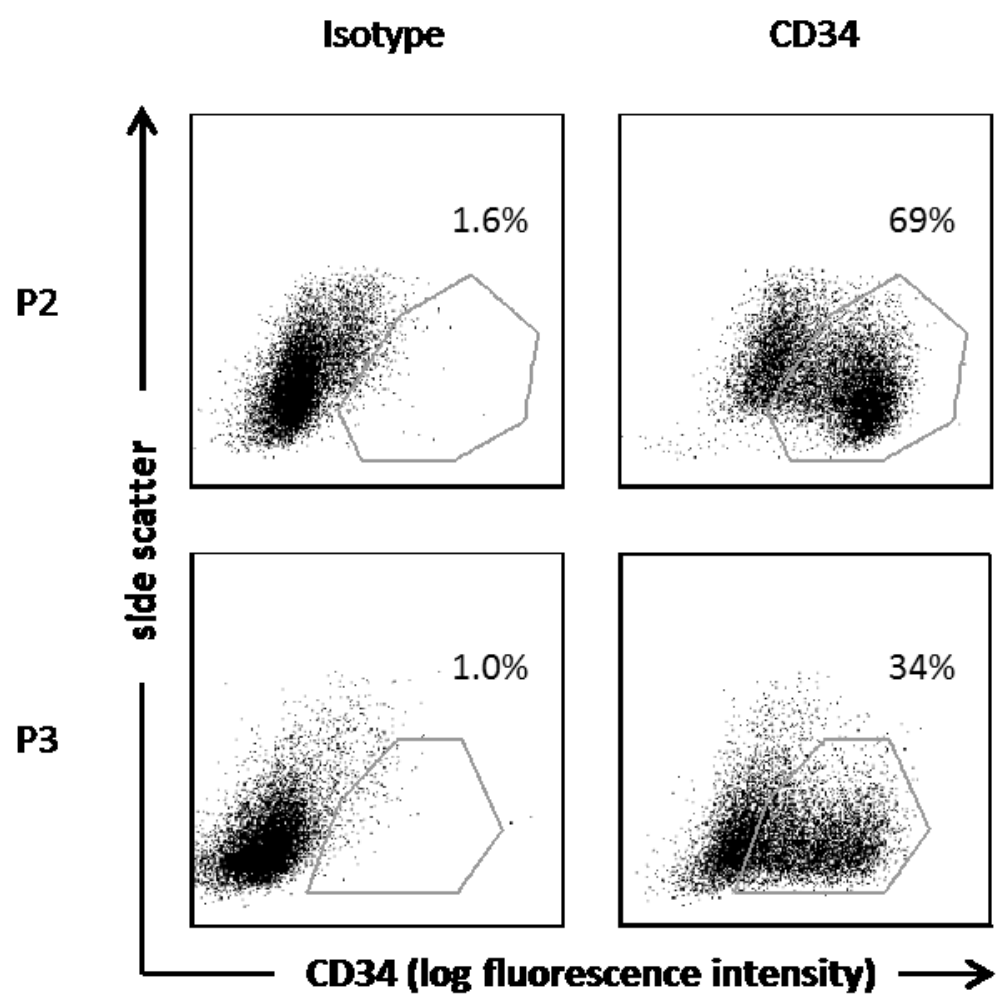

Figure S9. Gating strategy used to select CD $34^{+}$cells. 


\section{Experimental design}

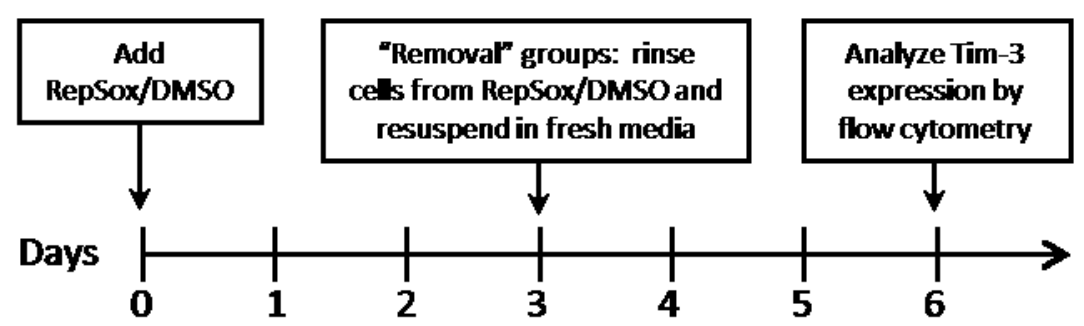

A

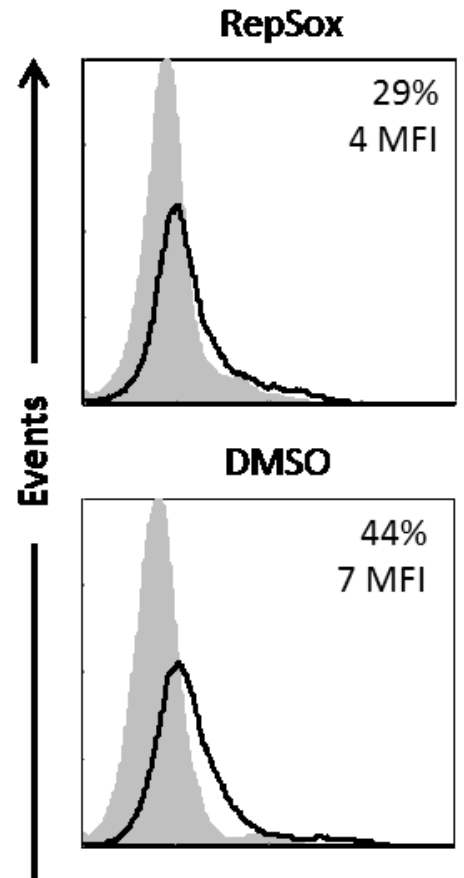

B

RepSox removal

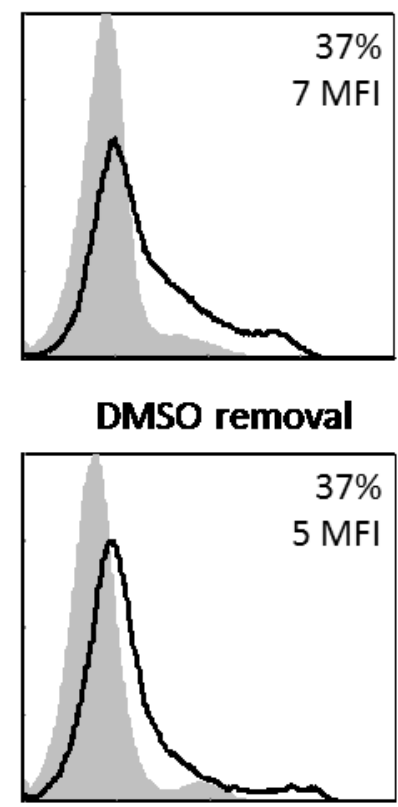

Tim-3 (log fluorescence intensity)

Figure S10. Increased proportion of Tim $-3^{+}$cells following removal of RepSox. 


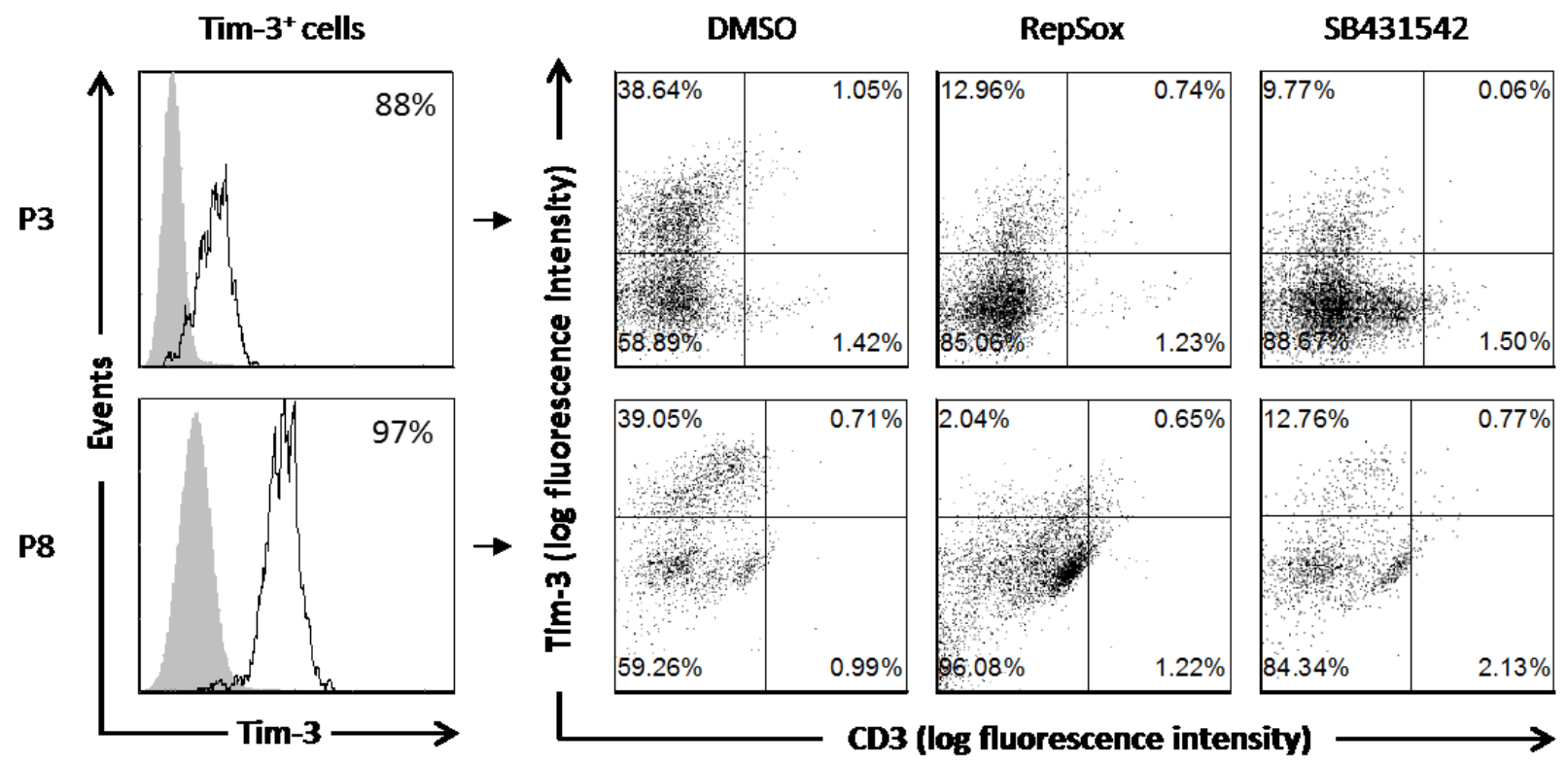

Figure S11. TGF- $\beta$ inhibitors RepSox and SB431542 reduce Tim-3 expression on AML and MDS cells. 


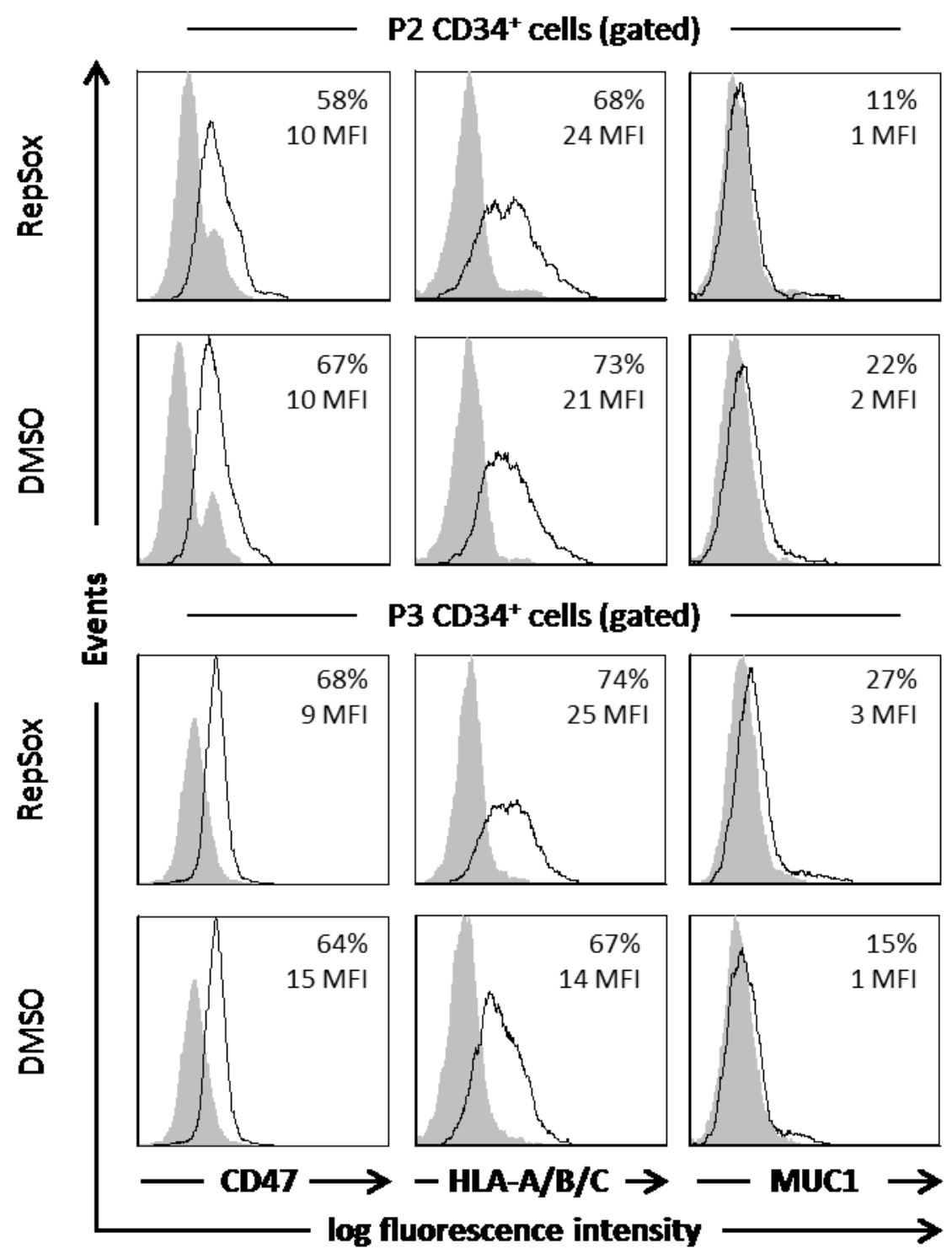

Figure S12. RepSox did not drastically alter CD47, HLA-A/B/C, or MUC1 expression on CD34 ${ }^{+}$AML and MDS cells. 


\section{References}

1 Dunn GP, Bruce AT, Ikeda $\mathrm{H}$, et al. Cancer immunoediting: from immunosurveillance to tumor escape. Nat Immunol 2002;3:991-998.

2 Zitvogel L, Tesniere A, Kroemer G. Cancer despite immunosurveillance: immunoselection and immunosubversion. Nat Rev Immunol 2006;6:715-727.

3 Jan M, Chao MP, Cha AC, et al. Prospective separation of normal and leukemic stem cells based on differential expression of TIM3, a human acute myeloid leukemia stem cell marker. Proc Natl Acad Sci USA 2011;108:5009-5014.

4 Kikushige $\mathrm{Y}$, Shima T, Takayanagi S, et al. TIM-3 is a promising target to selectively kill acute myeloid leukemia stem cells. Cell Stem Cell 2010;7:708-717.

5 van Stijn, A, van der Pol, MA, Kok, A, et al. Differences between the CD34+ and CD34blast compartments in apoptosis resistance in acute myeloid leukemia.Haematologica 2003;88: 497-508.

6 Costello RT, Mallet F, Gaugler B, et al. Human acute myeloid leukemia CD34+/CD38progenitor cells have decreased sensitivity to chemotherapy and Fas-induced apoptosis, reduced immunogenicity, and impaired dendritic cell transformation capacities. Cancer Res 2000;60:4403-4411.

7 Blankenstein T, Coulie PG, Gilboa E, et al. The determinants of tumour immunogenicity. Nat Rev Cancer 2012;12:307-313.

8 Kalos M, Levine BL, Porter DL, et al. T cells with chimeric antigen receptors have potent antitumor effects and can establish memory in patients with advanced leukemia. Sci Transl Med 2011;3:95ra73.

9 Brentjens RJ, Davila ML, Riviere I, et al. CD19-Targeted T Cells Rapidly Induce Molecular Remissions in Adults with Chemotherapy-Refractory Acute Lymphoblastic Leukemia. Sci Transl Med 2013;5:177ra38.

10 Khong HT, Restifo NP. Natural selection of tumor variants in the generation of "tumor escape" phenotypes. Nat Immunol 2002;3:999-1005.

11 Gajewski TF, Meng Y, Blank C, et al. Immune resistance orchestrated by the tumor microenvironment. Immunol Rev 2006;213:131-145.

12 Fourcade J, Sun Z, Benallaoua M, et al. Upregulation of Tim-3 and PD-1 expression is associated with tumor antigen-specific CD8+ T cell dysfunction in melanoma patients. J Exp Med 2010;207:2175-2186. 
13 Hodi FS, O'Day SJ, McDermott DF, et al. Improved survival with ipilimumab in patients with metastatic melanoma. N Engl J Med 2010;363:711-723.

14 Glaser, SP, Lee, EF, Trounson, E, et al. Anti-apoptotic Mcl-1 is essential for the development and sustained growth of acute myeloid leukemia. Genes Dev 2012,26:120125.

15 Noh KH, Kim BW, Song K, et al. Nanog signaling in cancer promotes stem-like phenotype and immune evasion. J Clin Invest 2012; 122:4077-4093.

16 Jordan CT, Upchurch D, Szilvassy SJ, et al. The interleukin-3 receptor alpha chain is a unique marker for human acute myelogenous leukemia stem cells. Leukemia 2000;14:1777-1784.

17 Majeti R, Chao MP, Alizadeh AA, et al. CD47 is an adverse prognostic factor and therapeutic antibody target on human acute myeloid leukemia stem cells. Cell 2009;138:286-299.

18 Spencer, JA, Ferraro, F, Roussakis, E, et al. Direct measurement of local oxygen concentration in the bone marrow of live animals. Nature 2014 [Epub ahead of print].

19 Ailles LE, Gerhard B, Hogge DE. Detection and characterization of primitive malignant and normal progenitors in patients with acute myelogenous leukemia using long-term coculture with supportive feeder layers and cytokines. Blood 1997;90:2555-2564.

20 Dontu G, Al-Hajj M, Abdallah WM, et al. Stem cells in normal breast development and breast cancer. Cell Prolif 2003;36 Suppl 1:59-72.

21 Reynolds BA, Weiss S. Generation of neurons and astrocytes from isolated cells of the adult mammalian central nervous system. Science 1992;255:1707-1710.

22 Eppert K, Takenaka K, Lechman ER, et al. Stem cell gene expression programs influence clinical outcome in human leukemia. Nat Med 2011;17:1086-1093.

$23 \mathrm{Noh}, \mathrm{KH}$, Lee, YH, Jeon, JH, et al. Cancer vaccination drives Nanog-dependent evolution of tumor cells toward an immune-resistant and stem-like phenotype. Cancer Res 2012;72:1717-1727.

24 Ichida JK, Blanchard J, Lam K, et al. A small-molecule inhibitor of tgf-Beta signaling replaces sox2 in reprogramming by inducing nanog. Cell Stem Cell 2009;5:491-503.

25 Gellibert F, Woolven J, Fouchet $\mathrm{MH}$, et al. Identification of 1,5-naphthyridine derivatives as a novel series of potent and selective TGF- $\beta$ type I receptor inhibitors. J Med Chem 2004;47:4494-4506. 
26 Hall BM, Fortney JE, Taylor L, et al. Stromal cells expressing elevated VCAM-1 enhance survival of B lineage tumor cells. Cancer Lett 2004;207:229-39.

27 Livark, KJ, and Schmittgen, TD. Analysis of relative gene expression data using realtime quantitative PCR and the 2 (-Delta Delta C (T)) method. Methods 2011;25:402-408.

28 Overton WR. Modified histogram subtraction technique for analysis of flow cytometry data. Cytometry 1988;9:619-626.

29 Eliasson P, Jonsson Jl. The hematopoietic stem cell niche: low in oxygen but a nice place to be. J Cell Physiol 2010;222:17-22.

30 Leon, J, Ferrandiz, N, Acosta, JC, et al. Inhibition of cell differentiation: a critical mechanism for MYC-mediated carcinogenesis? Cell Cycle 2009;8:1148-1157.

31 Salvatori, B, losue, I, Damas, ND, et al. Critical role of c-Myc in acute myeloid leukemia involving direct regulation of miR-26a and histone methyltransferase EZH2.Genes Cancer 2011;2:585-592.

32 Tavor, S, Eisenbach, M, Jacob-Hirsch, J, et al. The CXCR4 antagonist AMD3100 impairs survival of human AML cells and induces their differentiation. Leukemia 2008;22:2151-5158.

33 Huang, MJ, Cheng, YC, Liu, CR, et al. A small-molecule c-Myc inhibitor, 10058-F4, induces cell-cycle arrest, apoptosis, and myeloid differentiation of human acute myeloid leukemia. Exp Hematol 2006;34:1480-1489.

34 Hilton J. Role of aldehyde dehydrogenase in cyclophosphamide-resistant L1210 leukemia. Cancer Res 1984;44:5156-5160.

35 Chute JP, Muramoto GG, Whitesides J, et al. Inhibition of aldehyde dehydrogenase and retinoid signaling induces the expansion of human hematopoietic stem cells. Proc Natl Acad Sci USA 2006;103:11707-11712.

36 Ran D, Schubert M, Pietsch L, et al. Aldehyde dehydrogenase activity among primary leukemia cells is associated with stem cell features and correlates with adverse clinical outcomes. Exp Hematol 2009;37:1423-1434.

37 Gerber JM, Smith BD, Ngwang B, et al. A clinically relevant population of leukemic CD34(+)CD38(-) cells in acute myeloid leukemia. Blood 2012; 119:3571-3577. 
38 Pearce DJ, Taussig D, Simpson C, et al. Characterization of cells with a high aldehyde dehydrogenase activity from cord blood and acute myeloid leukemia samples. Stem Cells 2005;23:752-760.

39 Anderson AC. Tim-3, a negative regulator of anti-tumor immunity. Curr Opin Immunol 2012;24:213-216.

40 Inman, GJ, Nicolás FJ, Callahan JF, et al. SB-431542 is a potent and specific inhibitor of transforming growth factor-beta superfamily type I activin receptor-like kinase (ALK) receptors ALK4, ALK5, and ALK7. Mol Pharmacol 2002;62:65-74.

41 Rosenblatt J, Stroopinsky D, Luptakova K, et al. Muc1 inhibition reverses the poor immunogenicity of leukemia stem cells rendering them susceptible to immunotherapy. Biol Blood Marrow Tr 2012;18:S319.

42 Gletting DL, Kaplan DL. Extending hematopoietic stem cell survival in vitro with adipocytes. Biores Open Access 2013;2:179-185.

43 Kellner, J, Liu, B, Kang, Y, et al. Fact or fiction-identifying the elusive multiple myeloma stem cell. J Hematol Oncol 2013;6:1-10.

44 Hastings, WD, Anderson, DE, Kassam, N, et al. TIM-3 is expressed on activated human CD4+ $\mathrm{T}$ cells and regulates Th1 and Th17 cytokines. Eur $\mathrm{J}$ Immunol 2009;39:2492-2501.

45 McMahan, RH, Golden-Mason, L, Nishimura, MI, et al. Tim-3 expression on PD-1+ $\mathrm{HCV}$-specific human CTLs is associated with viral persistence, and its blockade restores hepatocyte-directed in vitro cytotoxicity. J Clin Invest 2010;120:4546.

46 Tang, D, \& Lotze, MT. Tumor immunity times out: TIM-3 and HMGB1.Nat Immunol 2012;13: 808-810.

47 Huang X, Bai X, Cao Y, et al. Lymphoma endothelium preferentially expresses Tim-3 and facilitates the progression of lymphoma by mediating immune evasion. J Exp Med 2010;207:505-520.

48 Larsen, DM. Evaluation of the TGF- $\beta$ Inhibitor RepSox on the Expression of Pluripotency Pathways in Murine and Bovine Cells [master's thesis]. Logan, UT: Utah State University; 2013. 
49 Wahl, SM, Swisher, J, McCartney-Francis, N, et al. TGF- $\beta$ : the perpetrator of immune suppression by regulatory T cells and suicidal T cells. J Leukoc Biol 2004;76:15-24.

50 Silva, J, Nichols, J, Theunissen, TW, et al. Nanog is the gateway to the pluripotent ground state. Cell 2009;138: 722-737.

$51 \mathrm{Hou}, \mathrm{P}, \mathrm{Li}, \mathrm{Y}$, Zhang, X, et al. Pluripotent stem cells induced from mouse somatic cells by small-molecule compounds. Science 2013;341:651-654.

52 Nair, R, Roden, DL, Teo, WS, et al. c-Myc and Her2 cooperate to drive a stem-like phenotype with poor prognosis in breast cancer. Oncogene 2013 [Epub ahead of print].

53 Burns, JM, Summers, BC, Wang, Y, et al. A novel chemokine receptor for SDF-1 and I-TAC involved in cell survival, cell adhesion, and tumor development. J Exp Med 2006;203:2201-2213.

54 Broxmeyer, HE, Kohli, L, Kim, CH, et al. Stromal cell-derived factor-1/CXCL12 directly enhances survival/antiapoptosis of myeloid progenitor cells through CXCR4 and Gai proteins and enhances engraftment of competitive, repopulating stem cells. J Leukoc Biol 2003;5:630-638.

55 Civini, S, Jin, P, Ren, J, et al. Leukemia cells induce changes in human bone marrow stromal cells. J Transl Med 2013;11:298.

56 Torossian, F, Anginot, A, Chabanon, A, et al. CXCR7 participates in CXCL12-induced CD34+ cell cycling through $\beta$-arrestin-dependent Akt activation. Blood 2014;123:191202.

$57 \mathrm{Kim}, \mathrm{HY}$, Oh, YS, Song, IC, et al. Endogenous stromal cell-derived factor-1 (CXCL12) supports autonomous growth of acute myeloid leukemia cells. Leuk Res 2013;37:566572.

58 Tavor, S, Petit, I, Porozov, S, et al. CXCR4 regulates migration and development of human acute myelogenous leukemia stem cells in transplanted NOD/SCID mice. Cancer Res 2004;64:2817-2824.

59 Chabanon, A, Desterke, C, Rodenburger, E, et al. A cross-talk between stromal cellderived factor- 1 and transforming growth factor- $\beta$ controls the quiescence/cycling switch of $\mathrm{CD} 34+$ progenitors through foxO3 and mammalian target of rapamycin. Stem Cells 2008;26:3150-3161.

$60 \mathrm{He}, \mathrm{M}$, Wang, QY, Yin, QQ, Tang, et al. HIF-1a downregulates miR-17/20a directly targeting p21 and STAT3: a role in myeloid leukemic cell differentiation. Cell Death Differ 2013;20: 408-418. 
61 Rehman, AO, and Wang, CY. CXCL12/SDF-1 $\alpha$ activates NF-kB and promotes oral cancer invasion through the Carma3/Bcl10/Malt1 complex. Int J Oral Sci 2009;1:105-118.

62 Hoesel, B, and Schmid, JA. The complexity of NF-kappaB signaling in inflammation and cancer. Mol Cancer 2013;12:1186.

63 Boyerinas, B., Park, S. M., Hau, A., et al. The role of let-7 in cell differentiation and cancer. Endocr Relat Cancer 2010;17:F19-F36.

64 Sampson, VB, Rong, NH, Han, J, et al. MicroRNA let-7a down-regulates MYC and reverts MYC-induced growth in Burkitt lymphoma cells. Cancer research 2007;67:97629770.

$65 \mathrm{He}, \mathrm{TC}$, Sparks, AB, Rago, C, et al. Identification of c-MYC as a target of the APC pathway. Science 1998;281:1509-1512.

66 Zhang, S, Li, Y, Wu, Y, et al. Wnt/B-catenin signaling pathway upregulates c-Myc expression to promote cell proliferation of P19 teratocarcinoma cells. Anat Rec (Hoboken) 2012;295:2104-2113.

67 Zheng, F, Li, H, Du, W, \& Huang, S. Role of hERG1 K+ channels in leukemia cells as a positive regulator in SDF-1a-induced proliferation. Hematology;16:177-184.

68 Wiener Z, Kohalmi B, Pocza P, et al. TIM-3 is expressed in melanoma cells and is upregulated in TGF- $\beta$ stimulated mast cells. J Invest Dermatol 2007;127:906-914.

69 Kim JS, Shin DC, Woo MY, et al. T cell immunoglobulin mucin domain (TIM)-3 promoter activity in a human mast cell line. Immune Netw 2012;12:207-212.

70 Chiba S, Baghdadi M, Akiba $\mathrm{H}$, et al. Tumor-infiltrating DCs suppress nucleic acidmediated innate immune responses through interactions between the receptor TIM-3 and the alarmin HMGB1. Nat Immunol 2012;13:832-842.

71 Ndhlovu LC, Lopez-Verges S, Barbour JD, et al. Tim-3 marks human natural killer cell maturation and suppresses cell-mediated cytotoxicity. Blood 2012;119:3734-3743.

72 Zhang Y, Ma CJ, Wang JM, et al. Tim-3 regulates pro- and anti-inflammatory cytokine expression in human CD14+ monocytes. J Leukoc Biol 2012;91:189-196.

73 Sakuishi K, Apetoh L, Sullivan JM, et al. Targeting Tim-3 and PD-1 pathways to reverse $T$ cell exhaustion and restore anti-tumor immunity. J Exp Med 2010;207:21872194. 
74 Ngiow SF, von SB, Akiba $\mathrm{H}$, et al. Anti-TIM3 antibody promotes T cell IFN-gammamediated antitumor immunity and suppresses established tumors. Cancer Res 2011;71:3540-3551.

75 Nishimura T, Kaneko S, Kawana-Tachikawa A, et al. Generation of rejuvenated antigen-specific $T$ cells by reprogramming to pluripotency and redifferentiation. Cell Stem Cell 2013;12:114-126.

76 Vizcardo, R, Masuda, K, Yamada, D, et al. Regeneration of human tumor antigenspecific T cells from iPSCs derived from mature CD8 ${ }^{+}$T cells. Cell Stem Cell 2013;12:3136.

77 Liu T, Guo Z, Yang Q, et al. Biochemical engineering of surface alpha 2-8 polysialic acid for immunotargeting tumor cells. J Biol Chem 2000;275:32832-32836.

78 Smits EL, Cools N, Lion E, et al. The Toll-like receptor $7 / 8$ agonist resiquimod greatly increases the immunostimulatory capacity of human acute myeloid leukemia cells. Cancer Immunol Immunother 2010;59:35-46.

79 Xing, F, Saidou, J, and Watabe, K. Cancer associated fibroblasts (CAFs) in tumor microenvironment. Front Biosci (Landmark Ed) 2009;15:166-179.

80 Cirri, P, and Chiarugi, P. Cancer associated fibroblasts: the dark side of the coin. Am J Cancer Res 2011;1:482-497. 
Chapter VII

\author{
Discussion
}




\section{Purpose}

The goal of this dissertation is to give readers interested in anti-cancer immune therapies an overview of historical events, conceptual frameworks, therapeutic options, technical challenges, and research opportunities. Dramatic advances in cell-engineering and reprogramming suggest patient-specific anti-CSC immunotherapies can now be developed even when CSCs in specimens are rare or missing. In general, therapeutic options are unlimited. Perhaps this dissertation can help students choose specific research agendas from the vast array of exciting opportunities.

Because immune therapies engineered in vitro have saved the lives of patients with a deadly leukemia, cancer researchers are now extremely optimistic. Historically, the evolution of anti-cancer strategies has been remarkable. Cancer researchers have shifted their attention from the chemical warfare agents of World War II to limb regeneration in salamanders, stem-cell technologies, and the engineering of immune cells as conceptual frameworks for cancer and immunology have evolved. This is truly an exciting time. Just during the course of this project, achievements (see Figure 5.1) have been profound. A Nobel Prize was awarded for clarifying how mature cells can be reprogrammed into pluripotent stem cells capable of regenerating any kind of specialized cell (Takahashi, 2006; Takahashi, 2007). Regarding cancer immune therapies, the FDA has approved Provenge, the first patient-specific cellular immunotherapy, to treat patients with advanced prostate cancer (Kantoff, 2010) and ipilimumab, an antibody that blocks the immune-checkpoint receptor CTLA-4, for the treatment of patients with metastatic melanoma (Hodi, 2010). The lives of leukemia and lymphoma patients have been saved by infusing their own $\mathrm{T}$ cells after these cells were genetically engineered in vitro to specifically attack their cancer cells (Kalos, 2011; Porter, 2011; Kochenderfer, 2012; 
Brentjens, 2013; Grupp, 2013). Major investments in cancer immunotherapy are being announced, including the National Cancer Institute (NCl)'s launch of the Cancer Immmunotherapy Trials Network (http://www.citninfo.org/). These kinds of achievements prompted Science to designate cancer immunotherapy as the 2013 "Breakthrough of the Year" (Couzin-Frankel, 2013).

Recently, the skillful use of RepSox and six other biologically potent chemicals was able to reprogram mouse fibroblasts to pluripotency without genetic engineering (Hou, 2013). This groundbreaking advance highlights the fantastic potential of in vitro cell manipulations and cell-fate control. Of note, the in vitro chemical reprogramming of cells may involve the same de-differentiation mechanisms conserved during evolution to generate and protect the primitive (normal) cells needed for tissue regeneration. These mechanisms, unfortunately, might be exploited to sustain populations of malignant cells when cancer cells are stressed. For example, Sall4 (Hou, 2013) - and possibly other genes activated during 7-factor chemical reprogramming of (normal) mouse cells to pluripotency - are activated during limb regeneration in salamanders (Neff, 2011). Furthermore, the same four embryonic transcription factors that reprogram mouse cells (Takahashi, 2006) also reprogram human cells (Takahashi, 2007). Diverse stimuli and environmental conditions can activate the same genes and signaling pathways, even across species, suggesting convergence on a few key mechanisms maintained over the course of evolution. Fortunately, critical molecular mechanisms are being clarified, and potent cell-engineering methods are being developed. As conceptual frameworks improve and additional mechanisms are being discovered, new therapeutic options are being identified which, in turn, are directing attention to specific technical obstacles that 
need to be overcome to engineer these therapies. Although these technical challenges can seem overwhelming, it is reassuring to know immune therapies are now saving the lives of cancer patients suffering from diseases recently considered "incurable" (Kalos, 2011; Porter, 2011; Kochenderfer, 2012; Brentjens, 2013; Grupp, 2013; Tran, 2014). Frustrated cancer researchers never abandoned their pursuit of immunologic strategies: More than 100 years ago they knew immune responses could (apparently randomly) eliminate tumors (Coley, 1893), and now they are rationally designing immune therapies that are predictably curing cancer patients.

\section{Rationale for studying RepSox}

Regeneration research prompted the decision to study how RepSox might affect AML cells. RepSox, an inhibitor of TGF- $\beta$ receptor 1 (aka ALK5) (Gellibert, 2004), was found to promote the reprogramming of normal cells to pluripotency by replacing two of the four critical factors previously identified: Sox2 and c-Myc (Ichida, 2009). In the context of the CSC theory, this observation seemed relevant for engineering patientspecific anti-cancer therapies. How might RepSox - a potent "reprogramming tool" - alter

malignant cells? Fortunately, when a patient's AML cells were exposed to RepSox in vitro, two effects were noted that could be useful for developing immuno-therapies: The decline in the proportion of CD34+ cells is slowed, and the decline in Tim-3 surface expression is accelerated (Jajosky, 2014). Thus, molecular tools that alter normal cells (Feng, 2009; Hou, 2013) may also alter tumor cells - and in ways potentially useful for engineering immune therapies.

Perhaps students will find it informative to review the rationale for considering the potential relevance of chemical "reprogramming tools" for anti-cancer therapy- 
development. In general, primary cancer cells tend to die or differentiate quickly in vitro. The ways RepSox affects normal cells (Ichida, 2009; Larsen, 2013) suggested RepSox might promote survival of primitive AML cells. That is, the reprogramming-associated effects of molecular tools on normal cells might predict their effects on tumor cells. Primitive tumor cells are critical according to the "CSC theory" which suggests stem-like cancer cells must be eliminated - in addition to the more mature and prevalent "bulk" tumor cells - to prevent disease-relapse. Thus, maintaining primitive tumor cells in vitro long enough to design, engineer, and test therapies was considered a vital technical goal. Given the ability of RepSox to promote cellular reprogramming, RepSox was evaluated as a "candidate tool" for AML therapy-development (Ichida, 2009). After exposing AML cells from leukapheresis specimens to RepSox, two effects were chosen for initial study: the survival of the CD34+ subset of AML cells and AML-cell expression of Tim-3.

AML stem cells (that can sustain disease and trigger relapse in patients - and engraft disease in immuno-deficient mice) are often enriched within the CD34 ${ }^{+}$subset (Bonnett, 1997; Eppert, 2011). That is, the CD34+ subset of AML cells in a patient's specimen generally contains the primitive LSCs that must be targeted to prevent relapse. Unfortunately, LSCs are difficult to study because they tend to rapidly die or differentiate in culture. However, the actions of RepSox suggested RepSox, even when used alone, might inhibit differentiation and maintain "stemness" (Ichida, 2009; Larsen, 2013). Thus, RepSox might facilitate engineering anti-AML immune therapies by helping to maintain primitive AML cells in vitro long enough so they can be used to identify therapeutic targets or evaluate immune-cell activation, antigen presentation, and candidate therapies. 
When expressed on the surface of cancer cells, the immune-checkpoint receptor Tim-3 can promote tumor-cell immune evasion. When expressed on the surfaces of the non-malignant antigen-presenting and immune-effector cells of cancer patients, Tim-3 induces immune tolerance, and Tim-3 levels can increase on these non-malignant cells as they interact with tumor cells, stromal cells, immuno-suppressive factors, metabolic dysregulations, and other components of cancer microenvironments (Huang, 2010; Baghdadi, 2013). In general, Tim-3 expression on cancer cells (Kikushige, 2010; Jan, 2011; Kikushige, 2012), tumor-associated endothelial cells (Huang, 2010), and antigenpresenting (Chiba, 2012) and immune-effector (Anderson, 2012) cells inhibits anti-tumor immune responses in patients with many types of cancers (see Tables 7.1 and 7.2). 


\begin{tabular}{|c|c|c|}
\hline Cancer type & Findings & References \\
\hline $\begin{array}{l}\text { Myelodysplastic } \\
\text { syndrome (MDS) }\end{array}$ & $\begin{array}{l}\text { Tim-3 is expressed by CD34+CD38 MDS cells; Tim- } 3 \text { is upregulated during } \\
\text { progression of MDS into AML }\end{array}$ & Yuda, 2013 \\
\hline $\begin{array}{l}\text { Acute myeloid leukemia } \\
\text { (AML) }\end{array}$ & $\begin{array}{l}\text { Tim-3 is expressed by AML LSCs (all subtypes except M3), but not normal } \\
\text { HSCs; Tim- } 3^{+} \text {, but not Tim-3 AML cells generated disease in } \\
\text { immunodeficient mice; Tim-3 may promote AML cell survival (by increasing } \\
\text { Mcl-1), immune evasion, and disease progression }\end{array}$ & $\begin{array}{l}\text { Kikushige, 2010; Jan, 2011; } \\
\text { Kikushige, 2012; } \\
\text { Yuda, 2013 }\end{array}$ \\
\hline $\begin{array}{l}\text { Chronic myeloid } \\
\text { leukemia (CML) and } \\
\text { chronic myelomonocytic } \\
\text { leukemia (CMML) }\end{array}$ & Tim-3 is expressed by primary $C M L$ and $C M M L$ cells & Yuda, 2013 \\
\hline Melanoma & $\begin{array}{l}\text { Tim-3 is over-expressed on melanoma cells and cell lines relative to normal } \\
\text { melanocytes; mast cells surrounding melanoma tumors also express Tim-3 }\end{array}$ & Wiener, 2007 \\
\hline Lung cancer & $\begin{array}{l}\text { Tim- } 3 \text { is expressed by non-small cell lung cancer cells ( } 26 / 30 \text { patients); } \\
\text { patients with Tim- } 3^{+} \text {tumors died sooner than patients with Tim- } 3^{-} \text {tumors }\end{array}$ & Zhuang, 2012 \\
\hline Cervical cancer & $\begin{array}{l}\text { Tim-3 is more highly expressed in patients with cervical cancer than } \\
\text { patients with cervical intraepithelial neoplasia and chronic cervicitis; high } \\
\text { Tim-3 expression correlated with greater metastasis, higher tumor grade, } \\
\text { and shorter overall survival }\end{array}$ & Cao, 2013 \\
\hline Osteosarcoma & $\begin{array}{c}\text { Tim-3+ tumor cells generally co-expressed EMT markers, such as Slug, Snail, } \\
\text { and Smad }\end{array}$ & Shang, 2013 \\
\hline $\begin{array}{l}\text { Various cancers of } \\
\text { histiocytic and dendritic } \\
\text { cell origin }\end{array}$ & $\begin{array}{l}\text { Tim-3 is expressed by neoplastic cells from patients with juvenile } \\
\text { xanthogranuloma (3/3), histiocytic sarcoma }(6 / 6) \text {, interdigitating dendritic } \\
\text { cell sarcoma (3/3), follicular dendritic cell sarcoma (1/4), langerhans cell } \\
\text { histiocytosis }(6 / 6) \text {, acute monocytic leukemia }(6 / 6) \text {, and hematodermic } \\
\text { tumors }(5 / 5)\end{array}$ & Dorfman, 2010 \\
\hline
\end{tabular}

Table 7.1. Human cancers that express Tim-3. 


\begin{tabular}{|c|c|c|}
\hline Disease State & Tim-3* Immune or Stromal Cells \& Key Findings & References \\
\hline \multicolumn{3}{|l|}{ Cancer } \\
\hline Melanoma & $\begin{array}{l}\text { NK cells; Higher Tim-3 expression on NK cells from melanoma patients than } \\
\text { NK cells from healthy controls; Tim- } 3^{+} \text {NK cells from melanoma patients were } \\
\text { functionally exhausted (impaired cytokine production, proliferation, and } \\
\text { cytotoxicity), and Tim-3 blockade improved NK cell cytotoxicity against } \\
\text { melanoma cell lines }\end{array}$ & Da Silva, 2014 \\
\hline $\begin{array}{l}\text { Acute myeloid leukemia } \\
\qquad \text { (AML) }\end{array}$ & $\begin{array}{l}\mathrm{CDB}^{+} \text {cytotoxic T cells; mice with AML develop functionally exhausted } \mathrm{CDB}^{+} \mathrm{T} \\
\text { cells that co-express Tim-3 and PD-1 }\end{array}$ & Zhou, 2011 \\
\hline $\begin{array}{l}\text { Follicular B cell non-Hodgkin } \\
\qquad \text { lymphoma }\end{array}$ & $\begin{array}{c}\text { T cells; IL-12 induces Tim-3 expression on T cells and promotes T cell } \\
\text { exhaustion }\end{array}$ & Yang, 2012 \\
\hline Lymphoma & $\begin{array}{l}\text { Endothelial cells dissected from human lymphomas; Tim- } 3^{+} \text {endothelial cells } \\
\text { suppressed activation of CD4+ helper } T \text { cells by activating the IL-6-STAT3 } \\
\text { pathway and inhibiting } T_{h} 1 \text { polarization }\end{array}$ & Huang, 2010 \\
\hline Melanoma & $\begin{array}{l}\text { Endothelial cells; melanoma cell-secreted TLR4 ligand induced endothelial } \\
\text { cells to express Tim-3; binding of melanoma cells to Tim- } 3^{+} \text {endothelial cells } \\
\text { promoted tumor cell proliferation and survival }\end{array}$ & Wu, 2010 \\
\hline \multicolumn{3}{|l|}{ Chronic Viral Infection } \\
\hline HIV & NK cells, T cells, and NKT cells: Tim- $3^{+}$immune cells were dysfunctional & $\begin{array}{l}\text { Jones, 2008; } \\
\text { Finney, } 2013\end{array}$ \\
\hline Hepatitis B & $\begin{array}{l}\text { NK cells: Tim-3+ NK cells were dysfunctional, and Tim-3 blockade improved } \\
\text { NK cell cytotoxicity and IFN- } \gamma \text { production }\end{array}$ & Ju, 2010 \\
\hline Hepatitis C & $\begin{array}{l}\text { CD8+ cytotoxic T cells: Tim- } 3^{+} \mathrm{T} \text { cells were dysfunctional and associated with } \\
\text { viral persistence and disease progression; Tim-3 blockade restored T cell } \\
\text { functions, including cytotoxicity against virally-infected hepatocytes }\end{array}$ & $\begin{array}{l}\text { Golden-Mason, 2009; } \\
\text { McMahan, 2010; } \\
\text { Vali, } 2010\end{array}$ \\
\hline
\end{tabular}

Table 7.2. Tim-3-expressing immune and stromal cells in the settings of cancer and chronic viral infections. 
Tim-3 expression is linked to AML LSCs (Kikushige, 2010; Kikushige, 2012) and is a useful marker for distinguishing Tim- $3^{+}$LSCs to be targeted from the Tim-3- HSCs (normal cells) to be spared (Jan, 2011). Tim-3 also highlights a potential confusion that can emerge concerning tumor-cell evolution and plasticity. Most often, CSCs are viewed as being able to give rise to more mature tumor cells. However, it has been found that tumor cells can evolve - even de-differentiate - into more stem-like cells in reaction to stresses like chronic inflammation (Landsberg, 2012) and immune-selection pressures (Noh, 2012b). These same stresses also seem to trigger the de-differentiation of normal cells that precedes the regeneration of tissues in primitive animals after injuries like amputations. Tumor cells have been found to acquire more stem-like phenotypes following serial rounds of "immune selection" in vivo (Lin, 2007; Noh, 2012a) and in vitro (Noh, 2012b). The acquisition of CSC-like characteristics induced by immune selection was replicated in vitro by transfection with Nanog (Noh, 2012b). That is, artificially induced Nanog signaling mimics the signaling induced when pathways are activated in tumor cells when they are attacked by immune cells - signaling that drives the tumor cells toward a stem-like, immune-resistant state (Noh, 2012b). Regarding leukemia cells, Tim-3 expression by AML cells may distort immunologic synapses between immune and AML cells, suppress immune-cell activation, promote immune evasion, contribute to leukemogenesis, and may identify a highly evolved LSC phenotype that can emerge in advanced disease (Kikushige, 2010; Kikushige, 2012). Thus, Tim-3 can be an important immuno-therapeutic target on both (malignant) AML cells and normal, but functionally impaired, immune cells. Thus, molecular tools like RepSox that reduce Tim-3 surface levels may be valuable for engineering immune therapies. 


\section{History of leukemia therapy}

Hippocrates used arsenic to treat leukemia in ancient Greece; its use continued until the $20^{\text {th }}$ century when it was replaced by radiation therapy. More than 100 years ago it was noted that the cancers of some patients spontaneously disappeared, and this regression of tumors seemed to be linked to fever-inducing infections. Thus, robust immune responses - induced by bacterial infections - were considered responsible for these spontaneous cures. Although radiation could improve the condition of leukemia patients, it caused leukemia in the radiologists who treated them. In 1913, the American Society for the Control of Cancer was founded to educate the public. Malignant diseases were so lethal and feared, cancer diagnoses were often withheld, denied, or ignored (like the taboo now linked to ebola diagnoses in some nations). In the 1930s, the Women's Field Army of volunteers initiated "a war on cancer." Their poster included the "Sword of Hope" - a symbol which remains part of the logo of the American Cancer Society. The handle of the sword consists of two intertwined serpents that symbolize medicine and science: healing and creativity. Regarding chemotherapy, soldiers exposed to the chemical warfare agent "mustard gas" during World War II were found to have low white blood cell counts. That observation essentially invented modern chemotherapy. Study of nitrogen mustard revealed it preferentially attacked rapidly dividing cells, including malignant cells.

In the 1940s, the chemical aminopterin was discovered in leukemia patients experiencing remission. So, Boston's Dr. Farber decided to use it therapeutically. Aminopterin is related to folic acid and blocks DNA replication in tumor cells. By 1950, Hitchings and Elion had carefully designed the compound 6-mercaptopurin specifically to 
disrupt DNA synthesis. Although this drug often induced remissions, leukemia patients later relapsed and died. While these classic studies and some early agents are still important, better anti-leukemia treatments are needed to prevent relapse and death. Although many leukemia drugs with diverse mechanisms of action are now available, the survival of residual LSCs that trigger relapse remains a challenge.

The potential of immunologic strategies was demonstrated when some leukemia patients were cured after being treated with monoclonal antibodies or bone-marrow cells from a donor who was not an identical twin of the patient (Thomas, 1999). In the case of allogeneic bone marrow transplants, a donor's genetically distinct immune cells can induce a graft-versus-leukemia immune reaction that blocks re-emergence of the host's LSCs (Thomas, 1999). Elimination of tumor cells by (activated) immune cells is the same mechanism that had been proposed to explain the spontaneous cancer regressions observed in the $19^{\text {th }}$ century (Coley, 1893).

Currently, AML relapses are still lethal, but researchers seem confident they will be able to predictably cure even patients with advanced disease using rationally designed, multi-pronged therapies that include a patient-specific immune strategy. In vitro cell-engineering techniques that exploit advances in cell reprogramming (Hou, 2013; Rais, 2013) and methods that repair dysfunctional antigen-presenting and immuneeffector cells can now generate immune cells that can be used therapeutically (following activation and expansion) (Hodi, 2010; Vizcardo, 2013; Themeli, 2013). Technical breakthroughs and new insights into cancer immunology are rapidly advancing cell engineering (Hou, 2013; Kalos, 2011; Porter, 2011) and the design of immuno-therapies (Hodi, 2010; Tran, 2014). As needed, even in the most problematic situations, these cell- 
engineering advances should enable researchers to manipulate mature cancer cells in routine specimens to generate the "CSC surrogates" that may be needed to develop patient-specific immune therapies. Cancer-induced pluripotent stem cells (iPSCs) have been generated from primary chronic myeloid leukemia cells (Kumano, 2012). Oct-4 has been shown to de-differentiate melanoma cells into CSC-like cells displaying reduced expression of melanocyte antigens, enhanced tumor-spheroid generation in vitro, and increased tumorigenicity in mice (Kumar, 2012). The successful reprogramming of tumor cells has been reassuring since patient-specific LSC-targeted immunotherapies may be needed to save the lives of some patients and engineering these therapies may require generating LSCs in vitro. That is, because some leukemia specimens contain few or no LSCs, a patient's mature leukemia cells may need to be de-differentiated into LSC-like cells. Thus, this project was influenced by the cell-repgrogramming breakthroughs achieved by tissue-regeneration researchers. Also of great interest, cell-culture advances had demonstrated how tumor microenvironments (Kinugasa, 2014) and bone-marrow factors, such as hypoxia (Yoshida, 2009; $\mathrm{Ng}, 2014$ ), bone-marrow stromal cells (Sutherland, 1990; Ailles, 1997), and adipocytes (Gletting, 2013), can help maintain stem and progenitor cells. However, cell-culture advances have not yet been fully exploited by, for example, integrating them with new reprogramming methods and molecular tools (Feng, 2009). The therapeutic vision for this project revealed how therapy-development may require not only the manipulation of primitive leukemia cells (to, for example, enhance immunogenicity and immune synapses in vitro) but also the manipulation of nonmalignant, but cancer-impaired, immune cells (to, for example, reverse defects responsible for immune tolerance). That is, reprogramming may be necessary when 
engineering therapies for some patients. Tumor cells may need to be reprogrammed into relevant, LSC-like surrogates (that can be studied, manipulated, and serve as targets in vitro), and dysfunctional immune cells may need to be reprogrammed (to repair them before they are activated and expanded). A possible cell-engineering strategy might involve repairing a leukemia patient's immune cells to restore cytolytic and/or antigenpresentation function (via reprogramming), activating effector immune cells against AML LSC antigens in co-culture, and then expanding these tumor-targeted immune cells before infusing them as therapy.

Collectively, experimental findings and conceptual frameworks for cancer and immunology have directed the attention to the potential value of de-differentiation and cell engineering. The technical obstacles encountered when with working with primary tumor cells in vitro have prompted cancer researchers to consider how regeneration scientists have successfully de-differentiated mature (normal) cells into pluripotent cells. To develop effective immune therapies for some leukemia patients, we may need to maintain - or generate - LSCs to design patient-specific anti-LSC therapies. That is, in response to the CSC theory and new data, attention has shifted from chemical warfare agents that target rapidly dividing bulk tumor cells to the stem cells and molecules involved in the regeneration of tissues in primitive animals. Thus, although pursuing different agendas, cancer and regeneration researchers now share a strong interest in de-differentiation.

Historically, advances in the conceptual frameworks for cancer seem to have driven progress. However, current cancer-immunology concepts and theories are so reliably predicting outcomes, clinical successes now seem to depend on technical advances. That is, current concepts seem adequate and comprehensive. For example, 
recent cancer "cures" induced by engineered T cells are validating the critical importance of "memory" T cells - as predicted. Researchers are envisioning effective therapies but need the methods and tools to engineer these therapies. Progress now seems to be "driven" by the methodical development of new techniques in research labs.

Finally, in retrospect, the history of cancer research is truly admirable and inspiring. It is comforting and reassuring to realize that efforts were not wasted: Many treatments that originally seemed like disappointing failures were actually impressive, rational agendas that are currently being reconsidered. Treatment "failures" have been informative. We now realize that in the context of "immunoediting" and "tumor-cell plasticity" almost any therapeutic strategy has the potential to make matters worse. To be effective long-term, anti-cancer therapies must be aggressive enough to eliminate a broad spectrum of tumor cells so that (1) CSCs do not have the opportunity to evolve into more lethal and death-resistant CSCs and (2) mature ("bulk") tumor cells do not have the opportunity to de-differentiate into CSCs. Thus, treatments initially considered ineffective may prove to be useful in the future as components of broader, multi-pronged strategies. Of note, many "conventional" or "non-immunologic" treatment options can induce beneficial immunologic effects. For example, physical removal of malignant cells via surgery or leukapheresis can reduce tumor-cell-induced immune-suppression. Radiation and some chemotherapies not only reduce the number of mature tumor cells but induce "immunogenic tumor-cell death" (Inoue, 2014; Ma, 2013) that can stimulate anti-cancer immune responses. Thus, multi-pronged anti-cancer therapies are attractive because, by exploiting the unique actions of diverse agents, they may be able to eliminate both mature and stem-like tumor cells. Of note, it is generally believed that patient-specific 
immune strategies may be required to eliminate a patient's CSCs. That is, CSCs are so death-resistant, they can survive even when exposed to radiation and chemotherapeutic drugs. Overall, the history of cancer research is truly inspiring: Each valiant effort has been informative, and past "failures" are being reconsidered for their potential value in the context of more comprehensive conceptual frameworks and multi-pronged strategies.

\section{Conceptual frameworks}

As conceptual frameworks for cancer, immunology, and tissue regeneration have evolved, themes and "lessons" have emerged that can provide guidance. For example, advances in diverse fields should be closely monitored so opportunities are not overlooked. As noted, in vitro de-differentiation - pioneered by regeneration researchers - may prove useful for engineering anti-cancer immunotherapies. De-differentiating a patient's tumor cells and non-malignant (but dysfunctional) antigen-presenting and immune-effector cells (Vizcardo, 2013; Themeli, 2013) may sometimes be required to engineer effective therapies. Interestingly, in humans, malignant cells may be the only cells that spontaneously de-differentiate - a mechanism that helps cancer cells survive when subjected to therapeutic or immunologic attack (Landsberg, 2012; Reiman, 2010).

Certain genes and signaling pathways seem especially critical for the generation, or survival, of normal stem cells and CSCs - and markedly different factors can induce activation of these genes and signaling pathways. Cell de-differentiation during in vitro reprogramming and amputation-induced limb regeneration in vivo are linked to the Wnt, Notch, and Hedgehog signaling pathways as well as activation of the Sall4 gene (Neff, 2011; Hou, 2013). Surface antigens like Tim-3 can be highly expressed on diverse cell types - including tumor (Wiener, 2007; Dorfman, 2010; Kikushige, 2010; Kikushige, 2012; 
Cao, 2013; Shang, 2013), endothelial (Huang, 2010), antigen-presenting cells (Chiba, 2012), and immune-effector cells (Anderson, 2012) in patients with a variety of cancers (see Tables 7.1 and 7.2). Furthermore, CAFs may also actively suppress immune cells (Silzle, 2004; Gieseke, 2013) as shown in Figure 3.6. Tim-3 is also expressed by functionally "exhausted" immune cells in patients with chronic viral infections (see Table 7.2) (Jones, 2008; Golden-Mason, 2009; Ju, 2010; McMahan, 2010; Vali, 2010; Finney, 2013). Thus, Tim-3 modulation may be a therapeutic strategy that may be useful for a variety of cancers and (chronic) viral diseases.

As emphasized previously, the most lethal tumor cells are stem-like cells that are hard to kill in vivo - but are hard to keep alive in vitro. Cancer cells that robustly grow in culture (that are like "HeLa cells") are extremely rare. Thus, researchers are working to find simple ways to maintain, or generate, a patient's lethal tumor stem cells in vitro in order to design, and test, strategies that can target them. That is, developing better in vitro ways to maintain a patient's CSCs - or generate CSC-like surrogates when necessary - is a major goal. Of note, in response to stress, normal stem and progenitor cells are protected by inflammation- and regeneration-related mechanisms that have been conserved during evolution. These same mechanisms are thought to protect primitive malignant cells that are exposed to chronic inflammation or therapeutic attack (Landsberg, 2013), and may provide guidance for maintaining primitive tumor cells in vitro. Of note, cancer's "unholy trinity" refers to three environmental conditions that support cancer cells: chronic inflammation, cytokines, and the transcription factor STAT3 (Li, 2011). These factors are thought to sustain the CSCs that maintain disease and trigger relapse following treatments that predominantly target bulk tumor cells. As the 
underlying mechanisms by which the "unholy trinity" (Li, 2011) maintains CSCs are clarified, they may be exploited to improve cell-culture techniques and guide the engineering of CSC-targeted immune therapies. However, the relationship between tumor cells and inflammation may be context-dependent. Chemotherapies that induce ICD are considered valuable components of anti-cancer therapies by inducing inflammation-related anti-cancer immune responses (Inoue, 2014; Ma, 2013).

Although finding better ways to maintain or generate a patient's stem-like cancer cells is still a challenge, recent successes with patient-specific anti-cancer immune therapies have validated current conceptual frameworks, and dramatic advances in cell engineering suggest all technical obstacles can eventually be overcome. Patients have achieved long-lasting remissions following adoptive transfer of their own T cells that have been engineered to express tumor-targeted chimeric-antigen receptors (CARs) ex vivo (Kalos, 2011; Porter, 2011; Kochenderfer, 2012; Brentjens, 2013). Following isolation, expansion, and reinfusion of cancer-reactive tumor-infiltrating $T$ cells, tumors regressed in a patient with metastatic cholangiocarcinoma (Tran, 2014). Immune-checkpoint blockade of CTLA4 stimulated immune responses and improved survival of patients with metastatic melanoma (Hodi, 2010). A genetically engineered oncolytic (cancer-lysing) strain of measles virus eliminated tumors in a patient with advanced "incurable" multiple myeloma (Russell, 2014). Although a patient's immune cells can become impaired ("exhausted" or unresponsive) as a cancer progresses, functionality can be restored after de-differentiating these cells (Vizcardo, 2013; Themeli, 2013; Nishimura, 2013), modulating immunoreceptors to unleash anti-tumor activity (Hodi, 2010), or manipulating the cells in other ways described in Chapter IV. Following reprogramming to pluripotency 
and re-differentiation, T cells were functionally "rejuvenated" and acquired elongated telomeres along with the capacity for significant expansion (Vizcardo, 2013; Themeli, 2013; Nishimura, 2013). Although conceptual frameworks highlight the complexity of malignant disease and the diverse dysregulations of local tumor microenvironments and the global immune system, dramatic clinical and cell-engineering successes have been achieved.

Also encouraging, in vitro techniques are effectively completing more tasks. Highly controlled in vitro conditions provide the opportunity to manipulate cells in diverse ways not possible in vivo where cancer-distorted microenvironments suppress anti-tumor immune responses via recruitment of myeloid-derived suppressor cells, release of soluble factors, and upregulation of inhibitory immune-checkpoint receptors that induce immune tolerance (Rabinovich, 2007). The in vitro evaluation and manipulation of cells in different contexts can be highly informative and inspire new treatment strategies. Methods for testing candidate anti-cancer therapies in vitro have correctly predicted their success in animals and humans (Peng, 2001; Carpenito, 2009; Milone, 2009; Noh, 2012b; Grosso, 2013). Also encouraging, although a patient's CSCs may be rare and elusive, in vitro engineered stem-like tumor cells that are not "bona fide CSCs" may be similar enough to the CSCs in vivo that they can serve as relevant therapeutic targets by virtue of immunologic cross-reactivity (Noh, 2012b). 
Worst-case clinical scenario: advanced disease with highly evolved, deathresistant, and treatment-refractory cancer cells

Before planning experiments, it was considered prudent to consider the "worstcase clinical scenario" when envisioning the engineering of a patient-specific anti-cancer immune therapy. What cell types might need to be altered, repaired, or activated? What cell manipulations and which methods might be required? The latest conceptual frameworks for cancer and immunology propose an evolving distribution of cancer cells ranging from death-resistant stem-like cells to their more mature progeny as well as an evolving distribution of dysfunctional antigen-presenting (Gabrilovich, 2004) and immuneeffector cells (Radoja, 2000; Frey, 2008; Lion, 2012; Stringaris, 2014). That is, both tumor and immune cells evolve as malignant disease advances, and the immune system becomes progressively dysregulated via a combination of tumor-cell immune evasion and immune-system tolerance that prevents adequate clearance of cancer cells (Kim, 2006). In highly evolved cancers, this emergence of multiple immunologic dysfunctions may require a multi-pronged therapy that combines patient-specific immune strategies with "conventional treatments." That is, a personalized approach may be required to effectively target each patient's unique immune-cell dysfunctions and CSC antigens. The "Immunoscore" cancer classification system proposes carefully examining the immune infiltrate within a patient's tumor to predict outcomes and guide therapeutic decisions (Galon, 2012; Fridman, 2012). As conceptual frameworks have improved, they have identified new mechanisms that explain how non-malignant antigen-presenting and immune-effector cells become dysfunctional in cancer (Radoja, 2000; Gabrilovich, 2004; Frey, 2008; Lion, 2012; Stringaris, 2014). Remarkably, dysfunctional immune cells can 
be "repaired" (Reiners, 2013; Vizcardo, 2013), reactivated (Hodi, 2010), and skillfully engineered (Themeli, 2013; Kalos, 2011; Porter, 2011) in vitro so they can be used therapeutically. For example, a melanoma patient's exhausted, tumor-infiltrating CD8 ${ }^{+}$ cytotoxic $T$ cells were functionally "rejuvenated" by first reprogramming them to pluripotency and then re-differentiating them back into T cells (Vizcardo, 2013; Themeli, 2013). The successful generation of normal HSCs from iPSCs (Lim, 2013; Suzuki, 2013; Amabile, 2013) suggests a cancer patient's non-malignant blood cells can be used to repopulate a patient's bone marrow with normal HSCs as needed. Of note, the latest findings and conceptual frameworks for cancer and immunology not only direct attention to the critical importance of the non-malignant cells of the immune system, but they help explain how these cancer-impaired cells can be repaired via de-differentiation (Vizcardo, 2013; Themeli, 2013). In general, as conceptual frameworks have advanced, they have identified new therapeutic options along with the technical tasks these strategies require. As noted, a "worst-case clinical scenario" (an advanced, highly evolved, and treatmentrefractory malignant disease) suggests a cancer patient's antigen-presenting and immune-effector cells may need to be reprogrammed to restore functionality (such as cytolytic activity and cytokine production) using techniques pioneered by regeneration researchers (Vizcardo, 2013; Themeli, 2013; Nishimura, 2013).

\section{Worst-case leukemia specimen: LSCs are rare and immunoevasive and the immune cells are dysfunctional}

In general, the blood or bone-marrow specimens taken from leukemia patients who are not severely immuno-compromised should contain the tumor and immune cells that are needed to engineer a patient-specific immunotherapy. However, it seems prudent to 
consider "the worst-case cancer specimen" and the challenges associated with maintaining primary cells ex vivo. Researchers should be aware, for example, that marked changes in the phenotypes of AML cells can occur as soon as the AML cells leave the bone marrow. Exposure to oxygen concentrations that are higher than the hypoxic levels in the bone marrow can reduce expression of CXCR4 and activate the AhR pathway. This may explain why the maintenance of primitive AML cells in vitro is improved by RepSox which may, conceivably (at least in some cells), induce CXCR4 expression via CXCL12 upregulation or via AhR pathway antagonism. In some studies, activation of the CXCL12/CXCR4 axis has been found to activate the PI3K/Akt "survival pathway" and to affect the AhR pathway. In general, the effects of the AhR pathway are cell-specific. Regarding AML, inhibition of the AhR pathway has been shown to improve the survival of primitive AML cells in vitro (Pabst, 2014).

Regarding the specimens from different AML patients, there is marked variation in the proportions and functionality of various immune cells as well as the proportions and phenotypes of CSCs. The proportions of LSCs, normal HSCs, antigen-presenting cells, immune-effector cells, and CAFs vary by AML subtype and stage, and by treatment history (Sarry, 2011; Wiseman, 2013; Kasper, 1999). Ideal specimens will contain adequate numbers of the CSCs to be targeted, normal HSCs to be spared, and functional APCs and tumor-reactive immune-effector cells - that is, cells that can simplify the engineering of a patient-specific therapy. In contrast, problematic specimens may contain few or no stem-like tumor cells (and immune cells that are highly dysfunctional). In that case, mature tumor cells may need to be de-differentiated into tumor cells that are sufficiently stem-like (Noh, 2012b; Kumar, 2012) to serve as in vitro surrogate targets for 
CSCs when engineering and evaluating candidate therapies. Figure 7.1 and Table 7.3 outline strategies and conditions that trigger cell de-differentiation. Immune-selection pressures (Reiman, 2010; Noh, 2012b); tumor-microenvironment conditions such as hypoxia (Sahlgren, 2008), CXCL12, and inflammation (Landsberg, 2012); and exposure to chemotherapy and radiation (Saxena, 2011; Sun, 2012; Chang, 2013) may drive tumor cells to undergo epithelial-to-mesenchymal transition (EMT) and/or acquire stem-like and immune- and death-resistant phenotypes. As immunogenic tumor cells are eliminated by the immune system, immune- and death-resistant cancer cells ("escape variants") can emerge and become predominant via natural selection (Dunn, 2002). Thus, tumor cells may need to be manipulated in vitro to increase their immunogenicity (to facilitate immune-cell activation in vitro) or modified in vivo using a (safe, non-toxic) systemic agent to decrease their death-resistance so they are susceptible to immunologic attack in vivo. 


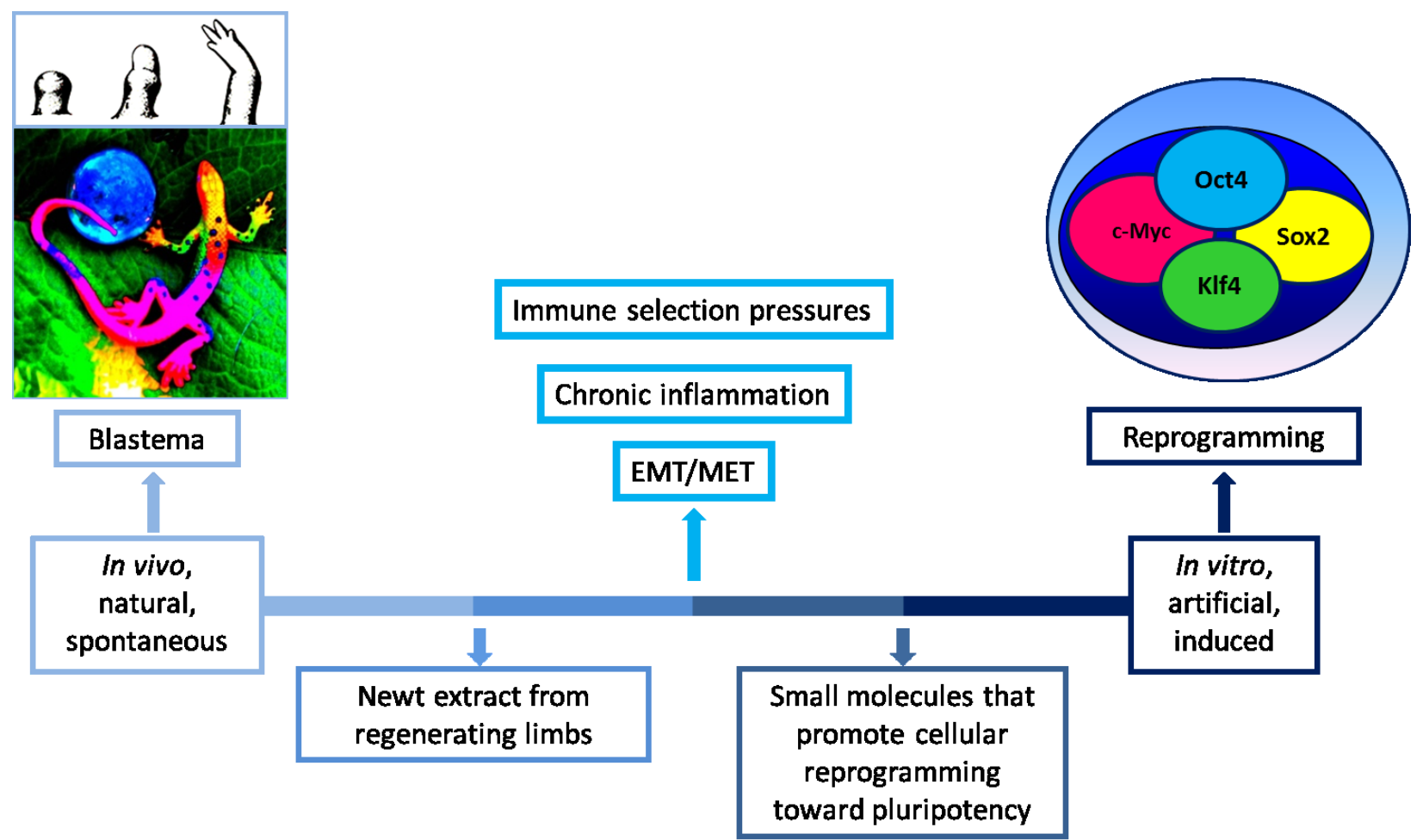

Figure 7.1. Spectrum of ways to induce de-differentiation. 


\begin{tabular}{|c|c|c|c|}
\hline & $\begin{array}{l}\text { De-differentiated or "converted" } \\
\text { cell types }\end{array}$ & Factors involved & $\begin{array}{l}\text { Degree of de-differentiation } \\
\text { or "conversion" }\end{array}$ \\
\hline $\begin{array}{c}\text { Regeneration post-injury } \\
\text { or amputation in primitive } \\
\text { animals }\end{array}$ & $\begin{array}{l}\text { Muscle (Nacu, 2014; Echeverri, } \\
\text { 2001), skin (Satoh, 2008), Schwann } \\
\text { cells (Tweedell, 2010), cartilage } \\
\text { (Tweedell, 2010), bone (Sousa, } \\
\text { 2011) }\end{array}$ & $\begin{array}{l}\text { Sall4 gene and other factors } \\
\text { linked to inflammation and } \\
\text { wound healing (Neff, 2011; } \\
\text { Monaghan, 2012) }\end{array}$ & $\begin{array}{l}\text { Partial reprogramming into } \\
\text { restricted progenitors } \\
\text { (Kragl, 2009; Stewart, 2012) }\end{array}$ \\
\hline $\begin{array}{l}\text { Immune-selection } \\
\text { pressures }\end{array}$ & $\begin{array}{l}\text { Cervical and colon cancer cells } \\
\text { (Noh, 2012) }\end{array}$ & $\begin{array}{l}\text { Activated Nanog signaling } \\
\text { increases the anti-apoptotic } \\
\text { factor Mcl-1 and resistance to } \\
\text { T cell-mediated death } \\
\text { (Noh, 2012) }\end{array}$ & $\begin{array}{l}\text { Conversion or evolution } \\
\text { toward stem-like, immune- } \\
\text { resistant tumor cells } \\
\text { (Noh, 2012) }\end{array}$ \\
\hline Chronic inflammation & $\begin{array}{l}\text { Melanoma cells } \\
\text { (Landsberg, 2012) }\end{array}$ & $\begin{array}{c}\text { TNF- } \alpha \\
\text { (Landsberg, 2012) }\end{array}$ & $\begin{array}{l}\text { Partial de-differentiation into } \\
\text { tumor stem-like cells } \\
\text { (Landsberg, 2012) }\end{array}$ \\
\hline $\begin{array}{l}\text { In vitro induced } \\
\text { pluripotent stem cell } \\
\text { (iPSC) reprogramming }\end{array}$ & $\begin{array}{l}\text { Many cell types including human } \\
\text { skin fibroblasts (Takahashi, 2007) } \\
\text { and blood cells (Loh, 2009) }\end{array}$ & $\begin{array}{l}\text { Oct4, Sox2, Klf4, and c-Myc } \\
\text { transcription factors } \\
\text { (Takahashi, 2007); small } \\
\text { molecules like RepSox (Feng, } \\
\text { 2009; Ichida, 2009; Hou, } \\
\text { 2013); Sall4 (Tsubooka, 2009) }\end{array}$ & $\begin{array}{l}\text { A small fraction of cells are } \\
\text { completely reprogrammed into } \\
\text { pluripotent stem cells; most } \\
\text { cells become partially } \\
\text { reprogrammed intermediates } \\
\text { (Hanna, 2010) }\end{array}$ \\
\hline $\begin{array}{l}\text { Epithelial-to- } \\
\text { mesenchymal transition } \\
\text { (EMT) }\end{array}$ & $\begin{array}{l}\text { Cancer cells that undergo EMT } \\
\text { acquire stem cell properties } \\
\text { (Mani, 2008) }\end{array}$ & $\begin{array}{l}\text { A variety of factors including } \\
\text { TGF- } \beta \text {, hypoxia, Snail, ZEB, and } \\
\text { immune-selection pressures } \\
\text { (Kalluri, 2009; Lamouille, 2014) }\end{array}$ & $\begin{array}{l}\text { Partial conversion into stem- } \\
\text { like tumor cells (Mani, 2008) }\end{array}$ \\
\hline
\end{tabular}

Table 7.3. Potential ways to induce cellular de-differentiation. 
As noted previously, when a patient-specific immune therapy is considered the best strategy, de-differentiation may be needed to repair a patient's defective immune cells (as well as generate CSC-like cells that can serve as surrogate targets in vitro). Immune-cell function might also be enhanced by increasing mTOR activity (via upregulation of the PI3K/Akt pathway) since mTOR has been found to improve immunecell function (Schmitz, 2008; Powell, 2012). Of note, RepSox can upregulate CXCL12, and CXCL12 can activate the PI3K/Akt/mTOR axis (Chen, 2012).

When a problematic cancer specimen contains few or no CSCs, more mature (more differentiated) tumor cells may need to be reprogrammed into stem-like cells. However, it is not clear how similar the CSC-like surrogates (to be used in vitro) must be to the actual disease-sustaining CSCs in vivo. Presumably, the more closely the CSClike cells used in vitro resemble the CSCs in vivo, the more relevant these cells will be when serving as surrogate targets for therapy-development. Of note, Noh found that a spectrum of stem-like cells having different degrees of "stemness" shared the same Nanog-mediated death-resistance to T-cell attack - and the greater their "stemness," the greater their death-resistance (Noh, 2012b). That is, although the "stemness" of these CSC-like cells varied, they shared the same therapeutic vulnerability as the most stemlike CSC. Furthermore, the less stem-like tumor cells could be made more stem-like (and more resistant to immune-cell attack) either through serial rounds of in vitro immuneselection pressure (via exposure to activated immune cells) or, more directly, by transfection with Nanog (Noh, 2012b). These observations suggest that the patient's most evolved and lethal CSCs may not be needed to design effective immune therapies - stem-like surrogates engineered in vitro may suffice. 
In general, if the reprogramming of cancer cells is needed (when only mature cancer cells are available in a specimen), the goal is to de-differentiate them into stemlike (CSC-like) cancer cells that adequately resemble the CSCs that can sustain disease and cause relapse. That is, the goal is not to de-differentiate mature cancer cells all the way to pluripotency. When salamanders regenerate tissues, the de-differentiation of cells is "partial" or "limited" in that the stem-like cells generated (to form the blastema) are "restricted progenitors" - not pluripotent stem cells (Christen, 2010; Garza-Garcia, 2010). Table 7.4 compares the partial, spontaneous (natural) de-differentiation of normal cells that occurs in vivo during regeneration with "full" de-differentiation ("artificially") induced in vitro by regeneration researchers when they generate pluripotent stem cells. Also notable, when human cancer cells are stressed - such as when exposed to toxic chemotherapeutic agents - they can de-differentiate into more death-resistant, stem-like phenotypes. That is, both normal cells (in primitive animals) and malignant cells (in humans) can spontaneously (and "partially") de-differentiate in vivo.

When considering conceptual frameworks and "worst-case scenarios" relevant for therapy-development, the potential need for, and value of, de-differentiating a patient's malignant cells and/or cancer-impaired immune cells in vitro becomes apparent. As noted, spontaneous cellular de-differentiation in primitive animals can generate critically important (normal) stem-like cells via mechanisms that can be exploited by human cancer cells to generate death-resistant CSCs. That is, de-differentiation is exploited by human tumor cells stressed by immunologic or therapeutic attack (cancer-cell immunoediting as a survival mechanism) and by normal cells stressed by injury (tissue regeneration as a survival mechanism). As noted, for in vitro therapy-development, de-differentiating both 
(malignant) tumor and (non-malignant) immune cells of a cancer patient may be required when dealing with problematic clinical scenarios and specimens. When the potential value of de-differentiation for therapy-development was considered, attention was directed to how a reprogramming tool like RepSox (Ichida, 2009) might alter AML cells.

\begin{tabular}{|c|c|}
\hline Regeneration in primitive animals & $\begin{array}{c}\text { Cellular reprogramming to } \\
\text { pluripotency }\end{array}$ \\
\hline in vivo & in vitro \\
\hline natural, spontaneous & artificial, induced \\
\hline no genetic manipulation & genetic or chemical engineering \\
\hline many unknown factors & few defined factors \\
\hline $\begin{array}{c}\text { partial reprogramming into restricted } \\
\text { progenitors }\end{array}$ & $\begin{array}{l}\text { complete reprogramming into } \\
\text { pluripotent stem cells }\end{array}$ \\
\hline cancer resistance & cancer risk \\
\hline
\end{tabular}

Table 7.4. Comparison of de-differentiation processes: the two extremes. 


\section{Most complex, unassuming therapeutic strategy}

In general, for patients with advanced cancers, complex, multi-pronged therapies may be needed that combine "conventional treatments" with immunologic strategies. Multi-pronged therapies might involve combinations of radio- and chemo-therapies, antibodies, activated antigen-specific anti-CSC immune cells, immune-stimulating agents, inhibitors of anti-apoptotic factor transcription, and/or many other potentially useful factors. These combination therapies will seek to target the entire spectrum of tumor cell phenotypes (in multiple ways) and immuno-suppressive microenvironments for problematic patients with advanced cancers (see Figure 4.1). Of course, physically removing tumor cells (via surgery or leukapheresis) can reduce immune suppression as well as reduce mechanical and/or hemodynamic problems caused by the presence of large numbers of malignant cells. Creating the patient-specific, anti-CSC immunologic components of multi-pronged strategies is a formidable challenge that may require extensive in vitro cell engineering that alters tumor cells and repairs, activates, and expands cancer-impaired immune cells. In the case of leukemias like AML, large numbers of tumor cells can be removed by leukapheresis which reduces the immunosuppression induced by these malignant cells. Chemotherapies that induce ICD should be beneficial (Zitvogel, 2008; Hodge, 2013; Ma, 2013; Inoue, 2014) via immune stimulation. Regarding cell engineering, developing immune strategies that can eliminate relapse-causing LSCs may require manipulating a patient's cells in different ways during different phases of therapy-development. In the most problematic leukemia scenario, none of the available malignant cells will be primitive enough to adequately represent the lethal disease-sustaining LSCs, and specimens will not contain sufficient numbers of 
normal HSCs. Because immune therapies with "immunological memory" must not destroy normal HSCs, LSCs and normal HSCs need to be compared and the status of each should be monitored when evaluating candidate therapies preclinically in vitro. These tasks require the ability to maintain pre-existing - or generate de novo (malignant) CSCs and (normal) HSCs which may be the most challenging in vitro agendas. Fortunately, advances in cell engineering suggest this is feasible. A remarkable example is a process by which cancer-impaired $\mathrm{T}$ cells are functionally "rejuvenated" via reprogramming and then expanded (Vizcardo, 2013; Themeli, 2013). Also, antigen-presenting cells have been successfully repaired and loaded with tumor antigens so they can be used therapeutically (Smits, 2009; Kantoff, 2010). In general, antibodies can be designed once suitable tumor-specific antigens have been identified. As emphasized, recent technical advances suggest that even the most challenging tasks related to immunotherapy-development can eventually be completed.

Despite dramatic advances in the engineering of therapeutic immune cells, studies suggest immune therapies may be inadequate when primitive tumor cells have evolved into extremely death-resistant cells. In that case, systemic agents may need to be administered that target the specific pro-survival mechanisms that protect these highly evolved CSCs (Igney, 2002; Vanneman, 2012). The death-resistance of tumor cells may involve changes in cell-surface antigens or changes in the intracellular transcription of critical molecules. For example, some death-resistant melanoma cells express CTLA-4. Thus, systemic administration of anti-CTLA- 4 antibodies may increase the susceptibility of these cancer cells to immunologic attack - as well as enhance the function of immune cells that express CTLA-4 (Laurent, 2013). Regarding the transcription problems inside 
cancer cells that increase their death-resistance, systemic agents can reduce the intracellular transcription of those anti-apoptotic factors that block immune-mediated killing, especially the killing of highly evolved CSCs (Vanneman, 2012). Another option is to use systemic agents that increase tumor-cell transcription of proapoptotic factors or factors like RIG-I that promote immune-mediated killing of tumor cells. Fortunately, processes like oligonucleotide production are highly advanced and can be used to produce agents that can be administered systemically to modulate the transcription of tumor-cell factors like RIG-I as well as antiapoptotic and proapoptotic factors. Cancer research now includes the study of intracellular mechanisms and transcription, and such studies are now explaining the anti-cancer actions of some agents for the first time. For example, one herb-derived anti-cancer chemical has been found to modulate transcription of the anti-apoptotic factor XIAP. Some malignant cells are death-resistant via XIAP overexpression, and by down-regulating XIAP, this herb isolate was able to promote the apoptosis of human cancer cells (Fang, 2012). In general, new insights have been gained regarding how herb-derived chemicals improve anti-cancer immunity or reduce tumor-cell death-resistance via studies that assessed cytokine production, immune-cell development, or the transcription of antiapoptotic or proapoptotic factors (Cheng, 2010; Peng, 2013). Herb-derived chemicals that favorably modulate key transcription mechanisms in tumor cells are candidates for systemic use since they have been used safely for centuries. In addition to the targeting of immune and tumor cells, agents have been evaluated that can be administered systemically to counteract a variety of immuno-suppressive factors within the tumor microenvironment (van den Boorn, 2013). 
Obviously, the expanding number of systemic options should promote the development of effective multi-pronged therapies.

Considering "worst-case scenarios" and the possible need for complex therapies helped guide this project. Current conceptual frameworks, past treatment failures, and the immunoediting and CSC theories identified potential therapy-development challenges in advance. For example, "working backwards" from a patient-specific therapeutic vision directed attention to the critical importance of maintaining and manipulating primitive tumor cells. CSCs available in specimens need to be maintained. If only mature cancer cells are available, they may need to be converted to CSC-like cells via chemical or genetic engineering or by in vitro use of immune-selection (Noh, 2012b) or chemotherapeutic pressures. Presumably, partial reprogramming of tumor cells is always an option. Furthermore, some specific genetic-engineering tasks might be among the options: for example, Nanog transfection - in the case of some cancers - might render tumor cells more stem-like by inducing the same cell changes induced by immune-cell attack (Noh, 2012b). In general, complex in vivo processes that protect and maintain normal and malignant stem cells are being replicated in vitro via skillful use of new cellengineering and cell-culture techniques. Learning how to generate and nurture the most lethal tumor cells may be necessary (so they can be studied) in order to find ways to kill them. Thus, factors and conditions considered "detrimental" because they sustain tumor cells in vivo are useful (for therapy-development) if they are also able to maintain cancer cells in vitro. For example, because "the unholy trinity" - STAT3, cytokines, and chronic inflammation (Li, 2011) - supports primitive tumor cells in vivo, these "unholy" factors may provide clues for how to maintain tumor cells effectively in vitro. Also, the same injury- 
induced and inflammation-related mechanisms that activate the genes and signaling pathways that protect the (normal) primitive cells (needed for regeneration after injury) may also generate and protect stem-like tumor cells in vitro. Thus, tumor necrosis factor (TNF)- $\alpha$ and other pro-inflammatory factors within tumor microenvironments that protect melanoma (and other types of tumor cells) from immune-cell attack by inducing their dedifferentiation (Landsberg, 2012) may prove to be useful for in vitro cell engineering.

In general, a cancer patients cells may need to be expanded in order to have adequate numbers of cells for in vitro studies and for the evaluation of candidate therapies preclinically. When engineered T cells, NK cells, and/or dendritic cells are to be infused as therapy, having sufficient numbers of these cells may require expanding these cell populations in vitro (Tran, 2014). In general, when considering multi-pronged immune therapies, both the technical challenges and therapeutic opportunities are numerous.

\section{Future directions related to RepSox}

Exploring how RepSox may affect immune-cell activation and Tim-3 expression

As emphasized, the actions of RepSox suggest this reprogramming tool might facilitate the engineering of patient-specific anti-cancer immune therapies. Questions to be answered are straightforward: How does RepSox affect Tim-3 expression on a cancer patient's non-malignant - but cancer-impaired - dendritic, NK, and T cells? Can reducing Tim-3 on AML cells improve immune-cell activation such as the spontaneous activation of $ү \delta T$ cells via co-culture of these cells? If so, by virtue of immunologic cross-reactivity and the existence of multiple tumor-cell antigens, will immune cells activated against RepSox-treated AML cells (whose Tim-3 expression has been eliminated) also be reactive against (lyse) the original AML progenitors (that were not exposed to RepSox)? 
Can RepSox help restore the function of cancer-impaired antigen-presenting and immune-effector cells?

How RepSox affects cancer-impaired immune cells, antigen presentation, and immune-cell activation warrants study for two reasons: (1) combined with other tools, RepSox can induce reprogramming (a process that can be used to restore the function of immune cells) and (2) used alone, RepSox can reduce expression of Tim-3 (a surface receptor that can disrupt the function of immune cells and inhibit immune-cell activation via distortion of immune synapses). As emphasized previously, the function of "exhausted" T cells from cancer patients can be restored ("rejuvenated") in vitro following reprogramming to pluripotency (Vizcardo, 2013; Themeli, 2013). Conceivably, combinations of RepSox and other chemicals could be used instead of genetic engineering to improve immune-effector-cell activity and antigen presentation just as combinations of RepSox and other chemical tools can replace genetic engineering when generating pluripotent stem cells (Hou, 2013). Perhaps "7-factor chemical reprogramming" (that fully de-differentiates normal cells) may provide guidance as to how RepSox and other chemicals can be used to alter a cancer patient's malignant cells and non-malignant immune cells in ways useful for therapy-development (Hou, 2013). It seems reasonable to conclude that since chemical engineering (Hou, 2013) and genetic engineering (Takahashi, 2007) have successfully de-differentiated (normal) cells to pluripotency, it should be possible to complete any desired in vitro cell-manipulation task. That is, since full reprogramming to pluripotency can be induced in vitro, what cellengineering task is not feasible? To promote cancer therapy-development, RepSox might be combined with some of the same factors that are already being used by regeneration 
researchers to manipulate normal cells (Feng, 2009). In general, new culture methods and research findings have identified a variety of potentially useful factors: the chemical reprogramming tools and "boosters" already being used with RepSox (Feng, 2009; Hou, 2013), physical conditions like hypoxia (Yoshida, 2009) and 3-D geometries, and cytokines and growth factors as well as a variety of support cells including adipocytes, osteoblasts, stromal cells, and even CAFs. Guidance is being provided by the steady flow of new advances. Although the possible combinations may seem overwhelming and unlimited, the opportunities are also numerous.

In this project, the effects of RepSox - and other reprogramming tools (Feng, 2009; Hou, 2013) - on antigen-presenting and immune-effector cells and on immune-cell activation were not studied, but warrant investigation. In vitro ways to measure immunecell activation are reviewed in Table 7.5. When evaluating how well immune cells react against tumor cells in vitro, adding chemotherapeutic agents like anthracyclines that induce "immunogenic" tumor cell death (Zitvogel, 2008; Ma, 2013; Inoue, 2014) might indicate whether some "conventional" anti-cancer drugs can further activate the immune system and work synergistically with immune strategies. 


\begin{tabular}{|c|c|}
\hline Immune Cell Activity or Marker & Methods of Evaluation \\
\hline Proliferation & $\begin{array}{c}\text { 3H-thymidine incorporation } \\
\text { CFSE dilution }\end{array}$ \\
\hline Cytokine production & $\begin{array}{c}\text { ELISA } \\
\text { Intracellular cytokine staining }\end{array}$ \\
\hline Expression of activation markers & T cell activation markers: CD107a, 0X40, 41-BB \\
\hline Cytotoxicity & $\begin{array}{c}\text { Immune-effector (NK or T) cell killing or lysis of } \\
\text { tumor cells; measure tumor cell viability or } \\
\text { their release of fluorescent dyes or radioactive } \\
\text { substrates like }{ }^{51} \text { Chromium }\end{array}$ \\
\hline Epitope specificity & Multimeric MHC-peptide complexes \\
\hline
\end{tabular}

Table 7.5. Measures of immune cell activation. 
Regarding Tim-3, functionally exhausted Tim- $3^{+}$immune cells can (eventually) appear in advanced malignant diseases and chronic viral infections. Impaired Tim-3expressing immune cells are associated with many cancers including melanoma (da Silva, 2014), AML (Zhou, 2011), and follicular B-cell non-Hodgkin's lymphoma (Yang, 2012). Exhausted Tim $-3^{+}$immune cells have been found in patients with chronic viral infections such as HIV (Jones, 2008; Finney, 2013), hepatitis B (Ju, 2010), and hepatitis C (Golden-Mason, 2009; McMahan, 2010; Vali, 2010). Of note, when expressed by endothelial cells isolated from human lymphoma tumors, Tim-3 was shown to suppress $\mathrm{CD}^{+}$helper T-cell activation by stimulating the IL-6/STAT3 pathway (Huang, 2010). Mesenchymal stem cells expressing galectin-9 (a Tim-3 ligand) have been shown to suppress T-cell proliferation (Gieseke; 2013). In general, Tim-3 signaling within tumor microenvironments is believed to promote tumor progression by suppressing the activity of infiltrating immune cells. That is, the Tim-3 receptor is not simply a marker of impaired ("exhausted") immune cells, but directly suppresses their activity. Because Tim-3 expression is a conserved molecular defect on the immune cells of patients with a variety of cancers and chronic infections, the ability of RepSox to reduce Tim-3 receptor levels in vitro (and, thereby, potentially restore and activate dysfunctional immune cells) may help researchers engineer therapies against a variety of diseases.

Exploring how RepSox may maintain primitive (normal) hematopoietic stem cells

Chapter VI discusses mechanisms that might explain how RepSox slows decay of $\mathrm{CD} 34^{+}$AML cells via its actions on c-Myc and CXCL12 (see Figure 7.2). Of note, CXCL12 is also known as "stromal cell-derived factor 1" (SDF-1). Considering the actions of RepSox on TGF- $\beta$ signaling and CXCL12, RepSox might also help maintain primitive 
(normal) hematopoietic stem cells in specially designed culture systems. For example, regarding the method developed by Khan, et al (Ali, 2014) - that is able to maintain and expand HSCs/HPCs in vitro - perhaps RepSox could replace use of "hKirre stromal-cell engineering" in their co-culture system. Why consider incorporating RepSox? RepSox seems potentially useful because Khan proposed that $h$ Kirre may promote the stemness and multipotency of umbilical cord blood cells (when co-cultured with stromal cells) via two hKirre-induced effects: promotion of CXCL12 and inhibition of TGF- $\beta$. Because RepSox can increase CXCL12 (Larsen, 2013; Jajosky, 2014) and inhibit TGF- $\beta$ signaling (Gellibert, 2004), RepSox might replace the hKirre manipulation of stromal cells in their co-culture system and, thereby, help maintain and expand HSCs/HPCs more easily.

Regarding RepSox-related mechanisms, exposing cells to RepSox in vitro might via CXCL12 upregulation - inhibit the death and differentiation of stem-like cells (that occurs spontaneously ex vivo) by inhibiting the AhR pathway. Of note, hypoxic microenvironments can preferentially sustain stem-like cells in vivo. Perhaps RepSox (via CXCL12) can enhance hypoxia inducible factor (HIF) activity in vitro, and increased HIF activity, in turn, has been shown to inhibit the AhR pathway. Of note, CXCL12 is a "hypoxia-inducible gene," and the CXCL12 promoter contains hypoxia inducible factor- $1 \alpha$ (HIF-1a) binding sites. Also, CXCL12 has been found to activate the PI3K/Akt pathway, and PI3K/Akt signaling contributes to activation of HIF-1a in the context of hypoxia. Research findings suggest CXCL12, HIF, and the AhR pathway are closely related. 


\section{Accelerated loss of Tim-3: \\ $\downarrow$ TGF- $\beta$ signaling}

Slowed decay of CD34+ AML cells:

个 CXCL12/SDF-1

$\downarrow$

$\uparrow$ Wnt signaling and/or $\uparrow$ Lin28 $\rightarrow \downarrow$ Let-7 family of microRNAs

$\downarrow$

个 c-Myc inhibits AML cell differentiation

$\downarrow$

$\uparrow$ microRNA-17, $\uparrow$ microRNA-20a

Figure 7.2: Potential mechanisms of RepSox action on AML cells. 
Of note, scientists feel "the balance" of CXCL12 and TGF- $\beta$ (or the "cross-talk" between these factors) controls a "cell-fate switch" that determines whether cells remain stem-like or differentiate. Researchers are interested in the mechanisms for cell-cycle regulation and self-renewal (how "the balance of cycling/quiescence" is controlled) because a more comprehensive understanding of these mechanisms will advance cell engineering (Lataillade, 2002; Chabanon, 2008). The existence of this 2-factor "cell-fate switch" is suggested by experimental observations (Chabanon, 2008) and may explain why RepSox is proving to be an especially useful and potent cell-engineering tool. Perhaps RepSox is unique: RepSox not only inhibits TGF- $\beta$, RepSox upregulates CXCL12 in (normal) mouse embryonic fibroblasts (Larsen, 2013) and in (malignant) AML cells (Jajosky, 2014). Of note, in this AML study, other TGF- $\beta$ inhibitors did not increase CXCL12 (Jajosky, 2014). By increasing CXCL12 mRNA levels, RepSox may be a unique TGF- $\beta$ inhibitor that can serve as a useful, and convenient, "CXCL12 research tool" that might replace, in some situations, use of expensive recombinant CXCL12 (aka SDF-1).

\section{Clarifying how RepSox affects CXCL12 and c-Myc}

One specific, potentially informative RepSox-related study could assess the effects of exposing AML cells to a combination of RepSox and the CXCR4 antagonist "AMD3100" for less than 5 days. Of note, short-term exposure (less than 5-days) to AMD3100 may be critical clinically. A "5-day cut-off" for AMD3100-exposed leukemia cells was identified by Kim, et al who observed two very different net effects induced by AMD3100 in vitro depending on whether the duration of AMD3100 exposure was greater than 5-days or less than 5-days (Kim, 2011). Of note, AMD3100 can be used clinically to mobilize primitive (normal or leukemic) blood cells from the bone marrow into the peripheral blood 
(Liles, 2003; Broxmeyer, 2005). AMD3100 reduces the ability of the CXCL12/CXCR4 axis to chemotactically retain primitive blood cells within the bone marrow where CXCL12secreting stromal cells attract CXCR4-expressing cells. AMD3100 reduces CXCL12 binding to CXCR4 by competitively binding to the CXCR4 surface receptor (Donzella, 1998; Rosenkilde, 2004). Thus, AMD3100 reduces the effects of CXCL12 mediated by the CXCL12/CXCR4 axis and is considered a CXCR4 antagonist, although it has some partial agonist activity in vitro (Zhang, 2002). However, a second CXCL12 receptor, CXCR7, has recently been discovered, and AMD3100 also binds to this receptor - but at a separate allosteric site not used by CXCL12 (Kalatskaya, 2009). The net effect is that during the first 5-days of exposure, AMD3100 (an "antagonist") may actually enhance the CXCL12-related effects induced by RepSox because AMD3100 can independently (noncompetitively) activate the CXCL12/CXCR7 axis. If activation of the CXCL12/CXCR7 axis (vs. the CXCL12/CXCR4 axis) is the key mechanism by which stem-cell maintenance and self-renewal are promoted, then AMD3100 might - during the first 5-days of combined exposure - enhance (instead of reduce) the ability of RepSox to maintain primitive AML cells. Of note, the ability of AMD3100 to potentially promote the maintenance and proliferation of primitive leukemia cells (via CXCL12/CXCR7 axis activation) is an issue - a warning - prudently raised by Kim et al because AMD3100 is used clinically to mobilize leukemia cells. They are concerned because AMD3100 has been assumed to be safe when viewed as a CXCL12 antagonist - before CXCR7, AMD3100-CXCR7 binding, and the actions of the CXCL12/CXCR7 axis had been identified (Kalatskaya, 2009). Based on their insights into CXCR7, Kim, et al have cautioned that use of AMD3100 could, conceivably, promote the maintenance or 
proliferation of primitive myeloid leukemia cells (Kim, 2011). If the addition of AMD3100 were to further enhance RepSox-induced maintenance of $\mathrm{CD} 34^{+} \mathrm{AML}$ cells (during the first 5 days of combined exposure), this would suggest that promoting CXCL12 in AML cells (or in both the stromal and AML cells) helps maintain primitive AML cells in vitro via the CXCL12/CXCR7 axis (since AMD3100 inhibits the CXCL12/CXCR4 axis). Of note, studies of normal endothelial cells suggest CXCL12 may maintain normal CD34 ${ }^{+}$ hematopoietic stem/progenitor cells via the CXCL12/CXCR7 axis (Torossian, 2014). Thus, RepSox may promote $\mathrm{AML} \mathrm{CD}_{3} 4^{+}$cell survival in part, or entirely, via the CXCL12/CXCR7 axis. The extent to which a patient's CD34 ${ }^{+}$AML cells express CXCR4 and/or CXCR7 may determine how well RepSox slows the decay of these AML cells. Exposing AML cells to combinations of AMD3100 and RepSox could clarify this issue.

To explain how the effects of RepSox on AML cells may be related to c-Myc, the CXCL12/CXCR4 axis, or the CXCL12/CXCR7 axis, normal stromal cells (from a healthy donor) and an AML patient's leukemia cells and CAFs can be exposed to combinations of (1) RepSox and c-Myc inhibitors such as 10058-F4 (Huang, 2006) and (2) RepSox and CXCR4 and CXCR7 agonists and antagonists. Since these research tools are available, it should be possible for interested researchers to clarify, in detail, RepSox-induced effects that are due to changes in CXCL12 and/or c-Myc.

RepSox studies may also be able to clarify how CAFs support tumor cells. Of note, Kojima, et al identified CXCL12 and TGF- $\beta$ as two factors critical for the evolution of stromal cells into tumor-promoting CAFs (Kojima, 2010): the same two factors believed to control the "cell-fate switch" (Chabanon, 2008). Activation of autocrine TGF- $\beta$ and CXCL12 signaling promotes conversion of stromal cells into tumor-promoting CAFs 
(Chabanon, 2008). Regarding fibroblasts from healthy animals (fibroblasts not exposed to cancer microenvironments), RepSox upregulates CXCL12 in mouse embryonic fibroblasts (Larsen, 2013) and inhibits TGF- $\beta$ signaling (Gellibert, 2004; Ichida, 2009). Thus, a potentially informative in vitro study might involve comparing how three different populations of stromal cells can support a patient's AML cells in vitro: (1) the canceraltered CAFs obtained from the (same) AML patient, (2) normal stromal cells (from a healthy source) that had been exposed to RepSox, and (3) normal stromal cells that had not been exposed to RepSox. In general, the actions of TGF- $\beta$ on stromal cells and cancer cells are complex and time-dependent. As noted by Caestecker, et al, during cancer progression, tumor cells often escape from the anti-proliferative effects of TGF- $\beta$ through acquired mutations or dysregulation of the TGF- $\beta$ signaling pathway (de Caestecker, 2000). Furthermore, Kojima, et al noted that some interactions between TGF- $\beta$ and CAFs involve the CXCL12/CXCR4 axis: In stromal fibroblasts, TGF- $\beta$ promotes TGF- $\beta$ and CXCL12 autocrine signaling loops (by promoting TGF- $\beta$ and CXCL12 production and inducing CXCR4 expression) which reciprocally cross-stimulate each other in a positive feedback manner to maintain the CAF phenotype (Kojima, 2010). Thus, experiments that compare how tumor cells are influenced by CAFs, normal stromal cells, and RepSox-treated stromal cells - and that skillfully exploit CXCR4 and CXCR7 antagonists, blocking antibodies, inhibitors, and other tools - may clarify how support cells promote the survival of CSCs. Learning how RepSox affects support cells may be as valuable as learning how RepSox can alter a cancer patient's tumor and immune cells. The evolution of support cells in cancer patients warrants study because 
microenvironments protect and sustain the primitive tumor cells that trigger cancer relapses.

Investigating how RepSox might help generate anti-AML immune-cell therapies

Since RepSox maintains primitive AML cells, upregulates CXCL12, and decreases

Tim-3, RepSox may promote the activation of immune cells by leukemia-cell antigens that could, under the proper circumstances, directly bind to immune-cell receptors. This seems feasible when the immunologic synapse between immune cells and AML cells is visualized in the context of prior research findings. One way to help readers visualize how RepSox may enhance the direct synapse between AML and immune cells involves the method developed by Gertner-Dardenne and colleagues (Gertner-Dardenne, 2012). In their co-culture system (which includes a "TCR agonist"), a patient's AML cells directly present antigens to immune cells, and this spontaneously activates the patient's $ү \delta \bar{T}$ cells against "tumor-associated" AML antigens (Gertner-Dardenne, 2012). This spontaneous activation of $\mathrm{T}$ cells triggered by the co-culture of $\mathrm{AML}$ and immune cells highlights the structure of the immunologic synapse. AML surface-antigens bind to (and activate) T-cell receptors like TCR and DNAM1 (Gertner-Dardenne, 2012). In the context of this AMLcell/immune-cell synapse, the actions of RepSox seem especially relevant and potentially useful. First, RepSox could promote the activation of immune cells against critically important stem-cell antigens by helping to maintain (in vitro) primitive CD34 ${ }^{+} \mathrm{AML}$ cells that are more likely to display LSC antigens than more mature AML cells. For those immune cells expressing the CXCR4 receptor, RepSox could enhance the physical aggregation of AML cells and immune cells via upregulation of AML-cell CXCL12 (Smith, 2013). That is, RepSox might promote chemoattraction and increase the number of 
immunologic synapses that form during co-culture of AML and immune cells. By decreasing Tim-3 expression, RepSox could reduce the distortion and dysfunction of the immunologic synapse that results when the Tim-3 receptor is present (Clayton, 2014).

Of note, it seems prudent to address a potential confusion that can emerge when Tim-3 is simply called an "LSC antigen." It is suggested here that Tim-3 on leukemia cells should, instead, be viewed as an "antigen on highly evolved LSCs." That is, LSCs that originally did not express Tim-3 may eventually display Tim-3 after leukemic disease has advanced and LSCs have evolved into more problematic and death-resistant LSCs. By simply referring to Tim-3 as an "LSC antigen," some AML cells that do not express Tim-3 might not be viewed as LSCs even though they are LSCs that have not yet evolved substantially and represent "early versions" of the original (disease-initiating) LSCs. It is assumed here that LSCs, like immune cells, evolve as malignant disease progresses, and that the more highly evolved LSCs can display Tim-3 (Zhou, 2011). Of note, the RepSox-treated AML cells whose Tim-3 expression was eliminated in this study still exhibited aldehyde dehydrogenase activity. If it is assumed that only Tim-3-expressing AML cells should be used to activate immune cells (by assuming only these AML cells are LSCs and only these cells express relevant stem-like tumor antigens), this could be counter-productive since Tim-3 can disrupt the immunologic synapse and, thereby, prevent in vitro immune-cell activation (Clayton, 2014).

Also for clarity, it is assumed here that the expression of AML cell-surface antigens is, in general, influenced by both the differentiation and evolution of AML cells. Noh and colleagues showed how cancer cells can change in response to "immune-selection 
pressures" and that a range of tumor cells with different phenotypes - reflecting different stages of evolution - could engraft disease in mice (Noh, 2012).

Regarding Tim-3 on tumor cells, Tim-3 has been linked to advanced stages of cancer and poor patient prognosis (Zhuang, 2012). This suggests Tim-3 expression emerges via tumor-cell immunoediting (cell plasticity and evolution) as malignant disease progresses (Zhou, 2011). Tim-3 receptors, in turn, can disrupt the immunologic synapse (Clayton, 2014), and this may explain how the more highly evolved cancer cells are better able to evade immune-cell attack.

The RepSox-related research option suggested here assumes that critical tumorassociated antigens on stem-like RepSox-exposed AML cells (that do not display Tim-3 surface receptors) can activate immune cells against primitive relapse-causing AML cells because expression of some LSC antigens should be unrelated to Tim-3 expression. Thus, exposing AML cells to RepSox may enhance immune-cell activation (by reducing AML-cell Tim-3 expression) as well as the aggregation (chemoattraction) of $A M L$ and CXCR4-expressing immune cells (by upregulating CXCL12). Of note, it is generally agreed that (1) the immunologic synapse is disrupted by Tim-3 and (2) chemoattraction involving CXCR4-expressing cells is enhanced by CXCL12 (Clayton, 2014; Kumar, 2006; Braza, 2010). Thus, RepSox may simultaneously enhance immune-cell activation and increase the number of immunologic synapses in co-culture systems (Smith, 2013).

As a specific example, RepSox might help generate therapeutic immune cells when incorporated into the co-culture system developed by Gertner-Dardenne and colleagues (Gertner-Dardenne, 2012). When their method is used to co-culture a patient's AML and immune cells - which exploits the "TCR agonist" bromohydrin pyrophosphate 
(BrHPP) - AML cells present AML-associated antigens to $\mathrm{Y} 9 \delta 2 \mathrm{~T}$ cells and spontaneously activate them (Gertner-Dardenne, 2012). F1F0-ATPase, PVR (CD155), and Nectin-2 (CD112) are some of the AML surface antigens thought to bind to, and activate, the yסTCR or DNAM1 (CD226) receptors on the T cells (Gertner-Dardenne, 2012; Wen-Li, 2012; Scotet, 2005). After expanding and infusing these activated $ү 9 \delta 2 \mathrm{~T}$ cells, survival improved in an animal model of AML (Gertner-Dardenne, 2012). Especially encouraging, these activated T cells had "memory" features which, potentially, can very effectively prevent cancer relapses (Gertner-Dardenne, 2012).

In the study by Gertner-Dardenne and colleagues, the phenotypic profile of the AML blasts was not specified. Thus, it is not known whether the patient-derived AML cells used to present tumor antigens to the immune cells were mature or stem-like. It seems prudent to assume that the phenotype of the AML cells can affect T-cell activation. That is, it seems reasonable to expect that the specific AML cell antigens presented to immune cells (to activate them) will depend on how stem-like the AML cells are. Presumably, the more stem-like the AML cells are, the more likely the activated immune cells will be reactive against those stem-like AML cells (the "minimal residual disease" cells) that can trigger disease-relapse. Thus, it seems prudent to exploit methods that can isolate, generate, and/or maintain stem-like AML cells so LSC-like AML cells are used to present antigens when activating immune cells. By first helping to maintain primitive $\mathrm{CD} 34^{+} \mathrm{AML}$ cells, RepSox should increase the likelihood that LSC antigens are presented to immune cells (by the RepSox-treated AML cells) when the immune cells are activated during coculture. That is, RepSox might enhance the $ү 9 \delta 2 \mathrm{~T}$-cell activation method of GertnerDardenne by supplying LSC-like AML cells for co-culture with the patient's immune cells. 
Remarkably, the $\mathrm{y} 9 \delta 2 \mathrm{~T}$ cells activated by AML-cell/immune-cell co-culture have the potential, in turn, to present antigens to, and activate, $\alpha \beta$ T cells (Brandes, 2009; Anderson, 2012). Exploring this technique for activating $\alpha \beta T$ cells - as well as other ways to activate NK cells and other $ү \delta$ T cells (not just $ү 9 \delta 2$ T cells) - seems especially appropriate for anti-AML therapy-development since AML cells can directly activate $\mathrm{\gamma} 9 \delta 2$ T cells. If RepSox can enhance $y 9 \delta 2$ T-cell activation, this may, in turn, facilitate the activation of other immune cells in subsequent phases of therapy-development.

Regarding molecular mechanisms, the "tumor-associated antigens" on AML cells thought to spontaneously activate $\mathrm{y} 9 \delta 2 \mathrm{~T}$ cells include Nectin, PVR, and F1F0-ATPase. Of course, when AML cells spontaneously activate $\gamma 9 \delta 2 \mathrm{~T}$ cells, other unknown AML cellsurface antigens may be involved. By eliminating synapse-disruption by Tim-3, perhaps RepSox can enable AML cells to more directly present AML antigens (like Nectin, PVR, and F1F0-ATPase) to receptors on immune cells.

Overall, it seems reasonable to investigate how RepSox might enhance the spontaneous activation of immune cells by AML cells in co-culture systems. Exploring diverse AML co-culture options might identify ways that a broad spectrum of $\alpha \beta$ T cells, NK cells, and other $ү \delta T$ cells (not just $ү 9 \delta 2$ T cells) can be activated against AML cells. As noted, just as AML cells serve as antigen-presenting cells to activate immune cells, Y9ס2 T cells activated by AML cells can, in turn, serve as antigen-presenting cells that can activate $\alpha \beta$ T cells (Deniger, 2013). Perhaps combinations of activated $\gamma 9 \delta 2$ T cells, AML cells, and chemical factors can be found that are able to activate a broad range of immune cells for therapeutic purposes (a spectrum of NK, $\gamma \delta T$ cells, and $\alpha \beta$ T cells) without, for example, using genetic engineering to insert chimeric antigen receptors 
(CARs). If genetic engineering cannot be avoided, it is reassuring that Deniger and colleagues have successfully activated polyclonal $ү \delta ~ T$ cells with broad anti-tumor capability using CAR technology and "artificial" antigen-presenting cells (Deniger, 2013). However, in view of the great diversity of AML cells among patients, co-culture methods that can exploit spontaneous immune-cell activation may eliminate the need to identify, in advance, specific AML "tumor-associated" surface-antigens - a task required when, for example, deciding what CAR insertions are to be genetically engineered.

To summarize this research option, the unique actions of RepSox might help generate anti-AML ү9ס2 T-cell therapies by, first, maintaining LSC-like AML cells that could be used to present stem-like AML antigens in co-culture systems that activate a patient's y9ס2 T cells. Then, either simultaneously or in a separate in vitro process, RepSox might improve y9ס2 T-cell activation when a patient's immune and (LSC-like) AML cells are co-cultured. That is, since RepSox maintains primitive CD34 ${ }^{+} \mathrm{AML}$ cells (that may display LSC-like tumor antigens), reduces Tim-3 expression (which disrupts immunologic synapses), and upregulates CXCL12 (that may increase chemoattraction and the number of immunologic synapses), RepSox may promote the co-culture activation of $ү 9 \delta 2 \mathrm{~T}$ cells against those primitive AML cells that trigger AML relapse. RepSox may be especially useful in advanced AML disease when LSCs have evolved to express Tim-3 cell-surface receptors which, in turn, disrupt immunologic synapses and inhibit immune-cell activation. 


\section{Future directions not involving RepSox}

Students should enjoy and embrace the improvements in conceptual frameworks for cancer, immunology, and tissue regeneration as well as the steady clarification of molecular mechanisms and signaling pathways. This progress is inspiring innovative therapeutic strategies which, in turn, are identifying key technical challenges and exciting research options. Immunology and regeneration research are directing attention to new tools and methods that can be used to engineer immunotherapies in vitro.

"7-factor" (chemical) reprogramming to pluripotency directs attention to the six other reprogramming factors that were used with RepSox (Hou, 2013). Perhaps these molecules can alter tumor-cell survival, differentiation status, immunogenicity, or other cellular features in ways useful for therapy-development. Like RepSox, three other chemicals were described as being especially useful: CHIR99021 (a glycogen synthase kinase 3 [GSK3- $\beta$ ] inhibitor), Forskolin (a cAMP agonist), and 3-deazaneplanocin A (“DZNep"; an S-adenosyl-homocysteine hydrolase inhibitor) (Hou, 2013). Perhaps combining one or more of these tools with RepSox would enhance the ability of RepSox to maintain the survival of primary $\mathrm{CD} 34^{+} \mathrm{AML}$ cells. The remaining three factors included valproic acid (a histone deacetylase [HDAC] inhibitor), tranylcypromine (a monoamine oxidase inhibitor), and "2i" treatment involving dual inhibition of mitogen-activated protein kinase signaling and GSK3- $\beta$. "Reprogramming boosters" were also identified including D4476 (an inhibitor of casein kinase I), 2-methyl-5-hydroxytryptamine (closely related to the neurotransmitter serotonin), basic fibroblast growth factor, prostaglandin E2, SRT1720 (a sirtuin 1 activator), and sodium butyrate (a HDAC inhibitor) (Hou; 2013). In general, because these agents and other molecular tools (Feng, 2009) promote cellular 
reprogramming (like RepSox), they may also alter the tumor and immune cells of AML patients in ways that can facilitate therapy-development.

Perhaps co-culturing a leukemia patient's tumor cells with support cells such as adipocytes, osteoblasts, stromal cells, or the patient's own CAFs would be helpful - or use of a 3-D cell-culture system. A trial-and-error approach may be unavoidable when trying to identify combinations of factors that might better maintain or alter the cells of AML patients. However, insights into signaling pathways and molecular mechanisms may predict worthwhile combinations. Regarding the activation of immune cells by tumor cells in vitro, adding chemotherapeutic agents like anthracyclines that induce immunogenic tumor-cell death (Inoue, 2014) may demonstrate whether or not some "conventional drugs" can enhance immune responses and work synergistically with immunologic strategies. In general, promising research options are unlimited due to the dramatic advances generated by cancer and regeneration research. When evaluating cell-engineering tools, effects will depend on the concentrations and combinations of factors and whether they are used simultaneously or sequentially. The steady discovery of new and exciting tools means setting priorities will be challenging. The ability to more predictably alter tumor and immune cells is increasing as evidenced by the impressive, rational design of "7-factor chemical de-differentiation" (Hou, 2013) along with reprogramming efficiencies near $100 \%$ (Rais, 2013). Because mature cells have been chemically reprogrammed to pluripotency (Hou, 2013), any desired in vitro cell manipulation now seems feasible. Still, despite dramatic advances, a basic challenge is likely to persist for years: the development of simple techniques that can maintain - or generate as needed - a patient's CSCs in vitro for therapy-development purposes. 


\section{Practical considerations for students}

In addition to describing specific ways RepSox might be used for in vitro cell engineering, therapy-development, and the clarification of mechanisms, this dissertation seeks to provide a useful overview. Although interactions between cancer cells and the immune system are numerous, complex, and even intimidating, students should appreciate how each newly identified mechanism provides an opportunity for intervention and therapeutic synergy.

When planning to engineer anti-cancer immune therapies in vitro, it seems worthwhile to consider those in vivo mechanisms that very effectively support tumor cells. In general, in vivo cancer microenvironments promote the maintenance and proliferation of CSCs while inhibiting the activation of immune cells. In contrast, in vitro conditions are "the reverse" in that it is difficult to maintain stem-like cancer cells ex vivo but effective immune-cell activation is possible in the absence of the immunologic restraints that exist in vivo. Understanding these differences can provide guidance for generating and/or maintaining (in vitro) the stem-like cancer cells that can serve as relevant "target cells" when designing antibody or immune-cell therapies.

Considering conceptual frameworks - like the CSC and immunoediting theories is also worthwhile. They explain why the immune-evasive and immune-suppressing CSCs that trigger relapse must be eliminated (as well as the more mature and prevalent "bulk" tumor cells) and that, because CSCs evolve, the sooner the better. To cure some patients, their own immune cells may need to be activated against CSC antigens and administered along with other personalized and conventional therapeutic strategies such as antibodies, immuno-modulating agents, surgery, chemotherapies, etc. 
Another worthwhile exercise is visualizing how the immunologic synapse is involved in immune-cell activation. When a cancer is highly advanced and a patient's specimens lack CSCs and immune cells that are functional ("reactive"), generating immune cells in vitro that are reactive against CSC antigens may be difficult. In this problematic scenario, it can be instructive to visualize how RepSox might enhance immunologic synapses in the specific context of co-culture systems that use a patient's AML cells to activate $y 9 \delta 2 \mathrm{~T}$ cells. The actions of RepSox suggest RepSox could facilitate in vitro activation of $\gamma 9 \delta 2$ cells against AML LSCs by enhancing (1) chemoattraction, (2) presentation of LSC antigens, and/or (3) activation of immune cells (by eliminating the adverse influence of Tim-3). That is, RepSox might enhance immunologic-synapse formation between AML and $ү \delta$ T cells. RepSox seems especially useful because AML cells directly present antigens to, and activate, $ү \delta \mathrm{T}$ cells. Thus, because RepSox (alone or in combination with other culture conditions and factors) may help maintain, or generate, primitive AML cells, RepSox may, thereby, help supply the primitive AML cells that can present (to immune cells) the LSC antigens that need to be targeted to prevent relapse. That is, by helping to provide LSC-like AML cells, RepSox may help activate immune cells so they are reactive against relapse-causing AML cells. During co-culture, RepSox may promote immunologic synapse formation and immune-cell activation by upregulating CXCL12 and/or by decreasing Tim-3 expression. Perhaps RepSox may enhance immune-cell activation against LSC antigens when incorporated into the coculture method developed by Gertner-Dardenne, et al (Gertner-Dardenne, 2012).

In addition to considering ways to improve the activation of T cells by AML cells, students should consider other challenges. It seems prudent to anticipate "worst-case 
scenarios" involving (1) tumor cells that are highly evolved and immune-resistant, (2) antigen-presenting and immune-effector cells that are markedly dysfunctional, and (3) specimens that may not contain adequate numbers of HSCs or primitive tumor cells. Fortunately, many new methods are becoming available, but students may still need to develop their own special techniques to accommodate the novel therapies they envision.

In the case of leukemia, for example, students may need to consider how they can best:

1. Obtain useful patient specimens that, ideally, contain the cells needed to engineer immunotherapies: primitive tumor cells to be targeted, normal HSCs to be spared, and dysfunctional antigen-presenting and immune-effector cells to be repaired. Leukapheresis specimens, obtained from patients with high white blood cell counts, contain billions of cells that are often discarded as waste. Aldefluor staining is a simple technique that distinguishes normal (ALDH ${ }^{\text {bright }}$ ) from AML (ALDH $\left.{ }^{\text {intermediate }}\right)$ stem/progenitor cells based on their unique staining patterns (Pearce, 2005; Gerber, 2012).

2. Maintain and manipulate tumor, antigen-presenting, and immune-effector cells as well as isolate normal HSCs for comparison purposes. The bone marrow stromal cells that have evolved in a cancer patient (CAFs) may help to maintain that patient's tumor and immune cells in vitro.

3. Develop co-culture methods that use primitive leukemia cells to activate immune cells so these immune cells are reactive against the stem-like leukemia cells that can trigger disease-relapse. 
4. Identify those anti-apoptotic factors in a patient's primitive tumor cells that inhibit tumor-cell killing when a candidate immune-cell therapy is tested in vitro.

5. Identify therapeutic agents (such as small molecules or oligonucleotides) that could safely be administered to patients to inhibit transcription of problematic antiapoptotic factors (or promote transcription of apoptotic factors) within tumor cells without harming normal HSCs.

6. Repair, activate, and/or expand cancer-impaired antigen-presenting and/or immune-effector cells (by, for example) reprogramming these cells, altering cellsurface antigens, enhancing tumor-antigen presentation, co-culturing immune and cancer cells with "TCR agonists" or via other strategies reviewed in Chapter IV.

7. Compare leukemia cells and normal HSCs to identify tumor-specific (or LSCspecific) cell-surface antigens or even notable intracellular differences.

8. Determine if some immune cells in a patient's specimen already react against tumor antigens. If so, select, or develop, a way to expand these cells. If reactive immune cells are not available, engineer (repair, activate, and expand) immune cells to be reactive against a suitable tumor-specific antigen.

9. Modulate tumor cells so they are more immunogenic or, more generally, improve immunologic-synapse formation in vitro to promote antigen-presentation and the activation of immune cells.

10. Identify how tumor cells impair immune-cell activation and acquire death-resistant phenotypes when attacked by immune cells or antibodies. 
11. Render tumor cells more stem-like in vitro, if needed, so they can serve as suitable in vitro therapeutic targets (by resembling the disease-sustaining LSCs that reside in vivo). This conversion into stem-like tumor cells may require chemical or genetic engineering or exposure to stresses involving serial rounds of immune-selection or chemotherapeutic "pressure."

12. Design multi-pronged immune therapies: combinations of antibodies, activated immune cells, agents that render tumor cells less death-resistant, factors that enhance immune responses (such as cytokines like IL-2), cytotoxic agents that induce immunogenic tumor cell death, and/or agents that mitigate the adverse impact of cancer-supporting microenvironmental cells and molecular factors that emerge as malignant disease progresses.

13. Evaluate candidate immune therapies pre-clinically in vitro.

Finally, students may find it instructive - and motivating - to consider how some therapeutic options that have already been developed might be combined to create rational, multi-pronged anti-cancer therapies. As noted previously, anti-cancer options are diverse and range from traditional in vivo tasks (the physical removal of malignant cells or systemic modulation of tumor cells, support cells, and the immune system) to new, more technically complex in vitro tasks (such as the skillful engineering of a patient's immune cells). For a blood cancer like AML, bulk tumor-cell removal is accomplished by leukapheresis which reduces the immune suppression induced by malignant cells. "Conventional chemotherapeutic drugs" that induce ICD (cytarabine, daunorubicin, 
mitomycin, etc.) not only kill cancer cells, but also enhance anti-cancer immune responses and promote tumor-cell apoptosis (Fredly, 2011). Cytokines like IL-2 can be administered systemically to enhance immunologic responses, and the impact of IL-2 may be enhanced by histamine dihydrochloride (Romero, 2009). Regarding immune-cell strategies, a patient's ү9ס2 T cells can be directly activated against a patient's stem-like AML cells via a co-culture system that exploits a TCR agonist (Gertner-Dardenne, 2012) - an in vitro process that might be enhanced by RepSox. After expansion, these $T$ cells could serve as the anti-LSC component of a multi-pronged therapy and provide long-term protection by functioning as "memory effector" immune cells. Ideally, NK cells, DCs and a broad spectrum of $\gamma \delta$ and $\alpha \beta$ T cells would be exploited. Regarding the deathresistance of stem-like cancer cells, primitive tumor cells might be rendered more susceptible to immune-cell attack by administering systemic agents that reduce the levels of key anti-apoptotic factors. For example, the antisense oligonucleotide "AEG35156" has been shown to reduce the levels of anti-apoptotic factor XIAP and, thereby, promote apoptosis of AML stem cells (Carter, 2011). Regarding systemic immune suppression induced by $T_{\text {regs, }}$ IL-2 diphtheria toxin fusion protein has promoted clearance of AML cells via depletion of Tregs (Bachanova, 2014). Regarding MDSCs, the agent "sunitinib," a receptor kinase inhibitor, has, in some cancer patients, enhanced the effectiveness of immune-based strategies and reversed immune suppression by reducing the numbers of MDSCs as well as Tregs (Ozao-Choy, 2009). An anti-CTLA-4 antibody (MDX-010) improved the ability of AML-derived DCs to activate $T$ cells against AML cells in an autologous culture system (Zhong, 2006). Envisioning such options illustrates how the complexity of malignant disease provides many opportunities for intervention. 


\section{Summary}

The interactions between cancer cells and the immune system are complex and fascinating. Remarkably, valuable insights into cancer and immunology have been prompted by very diverse and unusual observations. For example, infections, chemical warfare agents, and limb regeneration in salamanders have suggested that immune stimulation, immunogenic tumor-cell death, and cellular de-differentiation are biological phenomena worth considering when treating cancer patients. Perhaps some will find it counter-intuitive that the non-malignant cells of cancer patients are attracting intense attention: current therapeutic cell-engineering agendas involve (non-malignant) antigenpresenting and immune-effector cells, and researchers are also identifying the critical roles played by CAFs and other micorenvironmental factors that so effectively support malignant cells. Immune-cell therapies can be created in vitro using genetic engineering techniques (like CAR insertion) or co-culture methods that promote the spontaneous activation of immune cells by cancer cells. Multi-pronged therapies may be required that can simultaneously exploit diverse ways to promote the killing of tumor cells, enhance the immune system, or reverse the cancer-promoting features of cancer microenvironments.

More than 100 years after spontaneous tumor regressions were linked to concomitant infections, cancer researchers are now rationally engineering therapies that are predictably saving the lives of cancer patients (Kalos, 2011; Porter, 2011). Successful therapies include immune-based components (passively administered or actively induced) that eliminates those problematic, primitive cancer cells that can trigger relapse. Despite the complexity of malignant diseases, this is an historic era of cancer cures. Fantastic advances in cell engineering have facilitated the development of life-saving 
therapies. Given this historic context, students should feel fortunate. Students should confidently envision innovative therapies, eagerly confront technical challenges, and creatively exploit new tools and methods.

\section{References}

Ailles, L. E., Gerhard, B., \& Hogge, D. E. (1997). Detection and characterization of primitive malignant and normal progenitors in patients with acute myelogenous leukemia using long-term coculture with supportive feeder layers and cytokines. Blood, 90(7), 25552564.

Anderson, J., Gustafsson, K., Himoudi, N., et al. (2012). Licensing of үסT cells for professional antigen presentation: A new role for antibodies in regulation of antitumor immune responses. Oncoimmunology, 1(9), 1652.

Ali, I., Jiao, W., Wang, Y., et al. (2014). Ex Vivo Expansion of Functional Human UCBHSCs/HPCs by Coculture with AFT024-hkirre Cells. BioMed Research International, 2014.

Amabile, G., Welner, R. S., Nombela-Arrieta, C., et al. (2013). In vivo generation of transplantable human hematopoietic cells from induced pluripotent stem cells. Blood, 121(8), 1255-1264.

Anderson, A. C. (2012). Tim-3, a negative regulator of anti-tumor immunity. Current Opinion in Immunology, 24(2), 213-216.

Bachanova, V., Cooley, S., Defor, T. E., et al. (2014). Clearance of acute myeloid leukemia by haploidentical natural killer cells is improved using IL-2 diphtheria toxin fusion protein. Blood, 123(25), 3855-3863.

Baghdadi, M., \& Jinushi, M. (2013). The impact of the TIM gene family on tumor immunity and immunosuppression. Cellular \& Molecular Immunology. 11(1), 41-48.

Bonnet, D., \& Dick, J. E. (1997). Human acute myeloid leukemia is organized as a hierarchy that originates from a primitive hematopoietic cell. Nature Medicine, 3(7), 730737.

Brandes, M., Willimann, K., Bioley, G., et al. (2009). Cross-presenting human $ү \delta \bar{T}$ cells induce robust CD8+ $\alpha \beta \mathrm{T}$ cell responses. Proceedings of the National Academy of Sciences, 106(7), 2307-2312. 


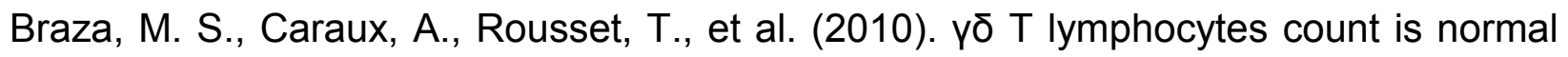
and expandable in peripheral blood of patients with follicular lymphoma, whereas it is decreased in tumor lymph nodes compared with inflammatory lymph nodes. The Journal of Immunology, 184(1), 134-140.

Broxmeyer, H. E., Orschell, C. M., Clapp, D. W., et al. (2005). Rapid mobilization of murine and human hematopoietic stem and progenitor cells with AMD3100, a CXCR4 antagonist. The Journal of Experimental Medicine, 201(8), 1307-1318.

Cao, Y., Zhou, X., Huang, X., et al. (2013). Tim-3 expression in cervical cancer promotes tumor metastasis. PloS One, 8(1), e53834.

Carpenito, C., Milone, M. C., Hassan, R., et al. (2009). Control of large, established tumor xenografts with genetically retargeted human T cells containing CD28 and CD137 domains. Proceedings of the National Academy of Sciences, 106(9), 3360-3365.

Carter, B. Z., Mak, D. H., Morris, S. J., et al. (2011). XIAP antisense oligonucleotide (AEG35156) achieves target knockdown and induces apoptosis preferentially in CD34+ 38- cells in a phase 1/2 study of patients with relapsed/refractory AML. Apoptosis, 16(1), 67-74.

Chabanon, A., Desterke, C., Rodenburger, E., et al. (2008). A Cross-Talk Between Stromal Cell-Derived Factor-1 and Transforming Growth Factor- $\beta$ Controls the Quiescence/Cycling Switch of CD34+ Progenitors Through FoxO3 and Mammalian Target of Rapamycin. Stem Cells, 26(12), 3150-3161.

Chang, L., Graham, P. H., Hao, J., et al. (2013). Acquisition of epithelial-mesenchymal transition and cancer stem cell phenotypes is associated with activation of the $\mathrm{PI} 3 \mathrm{~K} / \mathrm{Akt} / \mathrm{mTOR}$ pathway in prostate cancer radioresistance. Cell Death \& Disease, 4(10), e875.

Chen, G., Chen, S. M., Wang, X., et al. (2012). Inhibition of chemokine (CXC motif) ligand 12/chemokine (CXC motif) receptor 4 axis (CXCL12/CXCR4)-mediated cell migration by targeting mammalian target of rapamycin (mTOR) pathway in human gastric carcinoma cells. Journal of Biological Chemistry, 287(15), 12132-12141.

Cheng, C., \& Su, C. (2010). Tanshinone IIA inhibits Hep-J5 cells by increasing calreticulin, caspase 12 and GADD153 protein expression. International Journal of Molecular Medicine, 26(3), 379-385.

Chiba, S., Baghdadi, M., Akiba, H., et al. (2012). Tumor-infiltrating DCs suppress nucleic acid-mediated innate immune responses through interactions between the receptor TIM3 and the alarmin HMGB1. Nature Immunology, 13(9), 832-842. 
Christen, B., Robles, V., Raya, M., et al. (2010). Regeneration and reprogramming compared. BMC Biology, 8(1), 5.

Clayton, K. L., Haaland, M. S., Douglas-Vail, M. B., et al. (2014). T cell ig and mucin domain-containing protein 3 is recruited to the immune synapse, disrupts stable synapse formation, and associates with receptor phosphatases. The Journal of Immunology, 192(2), 782-791.

Coley, W. B. (1893). The treatment of malignant tumors by repeated inoculations of erysipelas: with a report of ten original cases. The American Journal of the Medical Sciences, 105(5), 487-510.

Couzin-Frankel, J. (2013). Cancer immunotherapy. Science, 342(6165), 1432-1433.

da Silva, I. P., Gallois, A., Baranda, S. J., et al. (2014). Reversal of NK cell exhaustion in advanced melanoma by Tim-3 blockade. Cancer Immunology Research, 2(5), 410-422.

de Caestecker, M. P., Piek, E., \& Roberts, A. B. (2000). Role of transforming growth factor- $\beta$ signaling in cancer. Journal of the National Cancer Institute, 92(17), 1388-1402.

Delgado-Martín, C., Escribano, C., Pablos, J. L., et al. (2011). Chemokine CXCL12 uses CXCR4 and a signaling core formed by bifunctional Akt, extracellular signal-regulated kinase (ERK) 1/2, and mammalian target of rapamycin complex 1 (mTORC1) proteins to control chemotaxis and survival simultaneously in mature dendritic cells. Journal of Biological Chemistry, 286(43), 37222-37236.

Deniger, D. C., Switzer, K., Mi, T., et al. (2013). Bispecific T-cells expressing polyclonal repertoire of endogenous $ү \delta$ T-cell receptors and introduced CD19-specific chimeric antigen receptor. Molecular Therapy, 21(3), 638-647.

Donzella, G. A., Schols, D., Lin, S. W., et al. (1998). AMD3100, a small molecule inhibitor of HIV-1 entry via the CXCR4 co-receptor. Nature medicine, 4(1), 72-77.

Dorfman, D. M., Hornick, J. L., Shahsafaei, A., et al. (2010). The phosphatidylserine receptors, $T$ cell immunoglobulin mucin proteins 3 and 4, are markers of histiocytic sarcoma and other histiocytic and dendritic cell neoplasms. Human Pathology, 41(10), 1486-1494.

Dubrovska, A., Hartung, A., Bouchez, L. C., et al. (2012). CXCR4 activation maintains a stem cell population in tamoxifen-resistant breast cancer cells through $A h R$ signalling.British Journal of Cancer, 107(1), 43-52.

Dunn, G. P., Bruce, A. T., Ikeda, H., et al. (2002). Cancer immunoediting: from immunosurveillance to tumor escape. Nature Immunology, 3(11), 991-998. 
Echeverri, K., Clarke, J. D., \& Tanaka, E. M. (2001). In vivo imaging indicates muscle fiber dedifferentiation is a major contributor to the regenerating tail blastema. Developmental Biology, 236(1), 151-164.

Eppert, K., Takenaka, K., Lechman, E. R., et al. (2011). Stem cell gene expression programs influence clinical outcome in human leukemia. Nature Medicine, 17(9), 10861093.

Fang, Y., Yu, Y., Hou, Q., et al. (2012). The Chinese herb isolate isorhapontigenin induces apoptosis in human cancer cells by down-regulating overexpression of antiapoptotic protein XIAP. Journal of Biological Chemistry, 287(42), 35234-35243.

Feng, B., Ng, J. H., Heng, J. et al. (2009). Molecules that promote or enhance reprogramming of somatic cells to induced pluripotent stem cells. Cell Stem Cell, 4(4), 301-312.

Finney, C. A., Ayi, K., Wasmuth, J. D., et al. (2013). HIV Infection Deregulates Tim-3 Expression on Innate Cells: Combination Antiretroviral Therapy Results in Partial Restoration. Journal of Acquired Immune Deficiency Syndromes, 63(2), 161-167.

Fredly, H., Ersvær, E., Gjertsen, B. T., et al. (2011). Immunogenic apoptosis in human acute myeloid leukemia (AML): primary human AML cells expose calreticulin and release heat shock protein (HSP) 70 and HSP90 during apoptosis. Oncology Reports, 25(6), 1549-1556.

Frey, A. B., \& Monu, N. (2008). Signaling defects in anti-tumor T cells. Immunological Reviews, 222(1), 192-205.

Fridman, W. H., Pagès, F., Sautès-Fridman, C., \& Galon, J. (2012). The immune contexture in human tumours: impact on clinical outcome. Nature Reviews Cancer, 12(4), 298-306.

Gabrilovich, D. (2004). Mechanisms and functional significance of tumour-induced dendritic-cell defects. Nature Reviews Immunology, 4(12), 941-952.

Galon, J., Pagès F., Marincola, F. M., et al. (2012). Cancer classification using the Immunoscore: a worldwide task force. Journal of Translational Medicine, 10, 1-9.

Garza-Garcia, A. A., Driscoll, P. C., \& Brockes, J. P. (2010). Evidence for the local evolution of mechanisms underlying limb regeneration in salamanders. Integrative and Comparative Biology, 50(4), 528-535. 
Gellibert, F., Woolven, J., Fouchet, M. H., et al. (2004). Identification of 1, 5-naphthyridine derivatives as a novel series of potent and selective TGF- $\beta$ type I receptor inhibitors. Journal of Medicinal Chemistry, 47(18), 4494-4506.

Gerber, J. M., Smith, B. D., Ngwang, B., et al. (2012). A clinically relevant population of leukemic CD34+ CD38- cells in acute myeloid leukemia. Blood, 119(15), 3571-3577.

Gertner-Dardenne, J., Castellano, R., Mamessier, E., et al. (2012). Human Vy9Vס2 T cells specifically recognize and kill acute myeloid leukemic blasts. The Journal of Immunology, 188(9), 4701-4708.

Gieseke, F., Kruchen, A., Tzaribachev, N., et al. (2013). Proinflammatory stimuli induce galectin-9 in human mesenchymal stromal cells to suppress T-cell proliferation. European Journal of Immunology, 43(10), 2741-2749.

Glettig, D. L., \& Kaplan, D. L. (2013). Extending human hematopoietic stem cell survival in vitro with adipocytes. BioResearch Open Access, 2(3), 179-185.

Golden-Mason, L., Palmer, B. E., Kassam, N., et al. (2009). Negative immune regulator Tim-3 is overexpressed on T cells in hepatitis $C$ virus infection and its blockade rescues dysfunctional CD4+ and CD8+ T cells. Journal of Virology, 83(18), 9122-9130.

Grosso, J. F., \& Jure-Kunkel, M. N. (2013). CTLA-4 blockade in tumor models: an overview of preclinical and translational research. Cancer Immunity, 13, 1-14.

Grupp, S. A., Kalos, M., Barrett, D., et al. (2013). Chimeric antigen receptor-Modified T cells for acute lymphoid leukemia. New England Journal of Medicine, 368(16), 15091518.

Hanna, J. H., Saha, K., \& Jaenisch, R. (2010). Pluripotency and cellular reprogramming: facts, hypotheses, unresolved issues. Cell, 143(4), 508-525.

Hodge, J. W., Garnett, C. T., Farsaci, B., et al. (2013). Chemotherapy-induced immunogenic modulation of tumor cells enhances killing by cytotoxic $\mathrm{T}$ lymphocytes and is distinct from immunogenic cell death. International Journal of Cancer, 133(3), 624-636.

Hodi, F. S., O'Day, S. J., McDermott, D. F., et al. (2010). Improved survival with ipilimumab in patients with metastatic melanoma. New England Journal of Medicine, 363(8), 711-723.

Hou, P., Li, Y., Zhang, X., et al. (2013). Pluripotent stem cells induced from mouse somatic cells by small-molecule compounds. Science, 341(6146), 651-654. 
Huang, X., Bai, X., Cao, Y., et al. (2010). Lymphoma endothelium preferentially expresses Tim-3 and facilitates the progression of lymphoma by mediating immune evasion. The Journal of Experimental Medicine, 207(3), 505-520.

Huang, M. J., Cheng, Y. C., Liu, C. R., et al. (2006). A small-molecule c-Myc inhibitor, 10058-F4, induces cell-cycle arrest, apoptosis, and myeloid differentiation of human acute myeloid leukemia. Experimental Hematology, 34(11), 1480-1489.

Ichida, J. K., Blanchard, J., Lam, K., et al. (2009). A small-molecule inhibitor of TGF- $\beta$ signaling replaces sox2 in reprogramming by inducing nanog. Cell Stem Cell, 5(5), 491503.

Igney, F. H., \& Krammer, P. H. (2002). Immune escape of tumors: apoptosis resistance and tumor counterattack. Journal of Leukocyte Biology, 71(6), 907-920.

Inoue, H., \& Tani, K. (2014). Multimodal immunogenic cancer cell death as a consequence of anticancer cytotoxic treatments. Cell Death \& Differentiation, 21(1), 3949.

Jajosky, A. N., Coad, J. E., Vos, J. A., et al. (2014). RepSox slows decay of CD34+ acute myeloid leukemia cells and decreases $T$ cell immunoglobulin mucin-3 expression. Stem Cells Translational Medicine, 3(7), 836-848.

Jan, M., Chao, M. P., Cha, A. C., et al. (2011). Prospective separation of normal and leukemic stem cells based on differential expression of TIM3, a human acute myeloid leukemia stem cell marker. Proceedings of the National Academy of Sciences, 108(12), 5009-5014.

Jones, R. B., Ndhlovu, L. C., Barbour, J. D., et al. (2008). Tim-3 expression defines a novel population of dysfunctional $\mathrm{T}$ cells with highly elevated frequencies in progressive HIV-1 infection. The Journal of Experimental Medicine, 205(12), 2763-2779.

Ju, Y., Hou, N., Meng, J., et al. (2010). T cell immunoglobulin-and mucin-domaincontaining molecule-3 (Tim-3) mediates natural killer cell suppression in chronic hepatitis B. Journal of Hepatology, 52(3), 322-329.

Kalluri, R., \& Weinberg, R. A. (2009). The basics of epithelial-mesenchymal transition. The Journal of Clinical Investigation, 119(6), 1420.

Kalos, M., Levine, B. L., Porter, D. L., et al. (2011). T cells with chimeric antigen receptors have potent antitumor effects and can establish memory in patients with advanced leukemia. Science Translational Medicine, 3(95), 95ra73-95ra73. 
Kantoff, P. W., Higano, C. S., Shore, N. D., et al. (2010). Sipuleucel-T immunotherapy for castration-resistant prostate cancer. New England Journal of Medicine, 363(5), 411-422.

Kasper, C., Ryder, W. D. J., Dürig, J., et al. (1999). Content of long-term culture-initiating cells, clonogenic progenitors and CD34+ cells in apheresis harvests of normal donors for allogeneic transplantation, and in patients with acute myeloid leukaemia or multiple myeloma. British Journal of Haematology, 104(2), 374-381.

Kikushige, Y., \& Akashi, K. (2012). TIM-3 as a therapeutic target for malignant stem cells in acute myelogenous leukemia. Annals of the New York Academy of Sciences, 1266(1), 118-123.

Kikushige, Y., Shima, T., Takayanagi, S. I., et al. (2010). TIM-3 is a promising target to selectively kill acute myeloid leukemia stem cells. Cell Stem Cell, 7(6), 708-717.

Kikushige, Y., Yuda, J., Shima, T., et al. (2013). TIM-3, a leukemia stem cell marker, plays a role in leukemic transformation through autocrine stimulatory signaling by its ligand, galectin-9. Blood, 122(21), 4196-4196.

Kim, R., Emi, M., Tanabe, K., et al. (2006). Tumor-driven evolution of immunosuppressive networks during malignant progression. Cancer Research, 66(11), 5527-5536.

Kim, H. Y., Hwang, J. Y., Oh, Y. S., et al. (2011). Differential effects of CXCR4 antagonists on the survival and proliferation of myeloid leukemia cells in vitro. The Korean Journal of Hematology, 46(4), 244-252.

Kinugasa, Y., Matsui, T., \& Takakura, N. (2014). CD44 expressed on cancer-associated fibroblasts is a functional molecule supporting the stemness and drug resistance of malignant cancer cells in the tumor microenvironment. Stem Cells, 32(1), 145-156.

Kojima, Y., Acar, A., Eaton, E. N., et al. (2010). Autocrine TGF- $\beta$ and stromal cell-derived factor-1 (SDF-1) signaling drives the evolution of tumor-promoting mammary stromal myofibroblasts. Proceedings of the National Academy of Sciences, 107(46), 2000920014.

Kragl, M., Knapp, D., Nacu, E., et al. (2009). Cells keep a memory of their tissue origin during axolotl limb regeneration. Nature, 460(7251), 60-65.

Kumano, K., Arai, S., Hosoi, M., et al. (2012). Generation of induced pluripotent stem cells from primary chronic myelogenous leukemia patient samples. Blood, 119(26), 62346242.

Kumar, S. M., Liu, S., Lu, H., et al. (2012). Acquired cancer stem cell phenotypes through Oct4-mediated dedifferentiation. Oncogene, 31(47), 4898-4911. 
Lamouille, S., Xu, J., \& Derynck, R. (2014). Molecular mechanisms of epithelialmesenchymal transition. Nature Reviews Molecular Cell Biology, 15(3), 178-196.

Landsberg, J., Kohlmeyer, J., Renn, M., et al. (2012). Melanomas resist T-cell therapy through inflammation-induced reversible dedifferentiation. Nature, 490(7420), 412-416.

Larsen, D. M. (2013). Evaluation of the TGF- $\beta$ inhibitor RepSox on the expression of pluripotency pathways in murine and bovine cells (Master's thesis). Available from ProQuest Dissertations and Theses database. (UMI Number: 1537216).

Lataillade, J. J., Clay, D., Bourin, P., et al. (2002). Stromal cell-derived factor 1 regulates primitive hematopoiesis by suppressing apoptosis and by promoting G0/G1 transition in CD34+ cells: evidence for an autocrine/paracrine mechanism. Blood, 99(4), 1117-1129.

Laurent, S., Queirolo, P., Boero, S., et al. (2013). The engagement of CTLA-4 on primary melanoma cell lines induces antibody-dependent cellular cytotoxicity and TNF- $\alpha$ production. Journal of Translational Medicine, 11(1), 108.

Lemay, C. G., Rintoul, J. L., Kus, A., et al. (2012). Harnessing oncolytic virus-mediated antitumor immunity in an infected cell vaccine. Molecular Therapy, 20(9), 1791-1799.

Li, N., Grivennikov, S. I., \& Karin, M. (2011). The unholy trinity: inflammation, cytokines, and STAT3 shape the cancer microenvironment. Cancer Cell, 19(4), 429-431.

Li, Y., \& Laterra, J. (2012). Cancer stem cells: distinct entities or dynamically regulated phenotypes? Cancer Research, 72(3), 576-580.

Liles, W. C., Broxmeyer, H. E., Rodger, E., et al. (2003). Mobilization of hematopoietic progenitor cells in healthy volunteers by AMD3100, a CXCR4 antagonist. Blood, 102(8), 2728-2730.

Lim, W. F., Inoue-Yokoo, T., Tan, K. S., et al. (2013). Hematopoietic cell differentiation from embryonic and induced pluripotent stem cells. Stem Cell Research \& Therapy, 4(3), 71.

Lion, E., Willemen, Y., Berneman, Z. N., et al. (2012). Natural killer cell immune escape in acute myeloid leukemia. Leukemia, 26(9), 2019-2026.

Loh, Y. H., Agarwal, S., Park, I. H., et al. (2009). Generation of induced pluripotent stem cells from human blood. Blood, 113(22), 5476-5479.

Ma, Y., Adjemian, S., Mattarollo, S. R., et al. (2013). Anticancer chemotherapy-induced intratumoral recruitment and differentiation of antigen-presenting cells. Immunity, 38(4), 729-741. 
Mani, S. A., Guo, W., Liao, M. J., et al. (2008). The epithelial-mesenchymal transition generates cells with properties of stem cells. Cell, 133(4), 704-715.

Matsubara, S., Ding, Q., Miyazaki, Y., et al. (2013). mTOR plays critical roles in pancreatic cancer stem cells through specific and stemness-related functions. Scientific Reports, 3, 3230 .

McMahan, R. H., Golden-Mason, L., Nishimura, M. I., et al. (2010). Tim-3 expression on PD-1+ HCV-specific human CTLs is associated with viral persistence, and its blockade restores hepatocyte-directed in vitro cytotoxicity. The Journal of Clinical Investigation, 120(12), 4546-4557.

Milone, M. C., Fish, J. D., Carpenito, C., et al. (2009). Chimeric receptors containing CD137 signal transduction domains mediate enhanced survival of T cells and increased antileukemic efficacy in vivo. Molecular Therapy, 17(8), 1453-1464.

Monaghan, J. R., Athippozhy, A., Seifert, A. W., et al. (2012). Gene expression patterns specific to the regenerating limb of the Mexican axolotl. Biology Open, 1(10), 937-948.

Nacu, E., Tazaki, A., Joven, A., et al. (2014). Fundamental differences in dedifferentiation and stem cell recruitment during skeletal muscle regeneration in two salamander species. Cell Stem Cell, 14, 1-14.

Nanki, T., \& Lipsky, P. E. (2000). Cutting edge: stromal cell-derived factor-1 is a costimulator for CD4+ T cell activation. The Journal of Immunology, 164(10), 5010-5014.

Neff, A. W., King, M. W., \& Mescher, A. L. (2011). Dedifferentiation and the role of sall4 in reprogramming and patterning during amphibian limb regeneration. Developmental Dynamics, 240(5), 979-989.

Ng, K. P., Manjeri, A., Lee, K. L., et al. (2014). Physiologic hypoxia promotes maintenance of CML stem cells despite effective BCR-ABL1 inhibition. Blood, 123(21), 3316-3326.

Nishimura, T., Kaneko, S., Kawana-Tachikawa, A., et al. (2013). Generation of rejuvenated antigen-specific $T$ cells by reprogramming to pluripotency and redifferentiation. Cell Stem Cell, 12(1), 114-126.

Noda, M., Omatsu, Y., Sugiyama, T., Oishi, S., Fujii, N., \& Nagasawa, T. (2011). CXCL12CXCR4 chemokine signaling is essential for NK-cell development in adult mice. Blood, 117(2), 451-458.

Noh, K. H., Kim, B. W., Song, K. H., et al. (2012). Nanog signaling in cancer promotes stem-like phenotype and immune evasion. The Journal of Clinical Investigation, 122(11), 4077. 
Ozao-Choy, J., Ma, G., Kao, J., et al. (2009). The novel role of tyrosine kinase inhibitor in the reversal of immune suppression and modulation of tumor microenvironment for immune-based cancer therapies. Cancer Research, 69(6), 2514-2522.

Pabst, C., Krosl, J., Fares, I., et al. (2014). Identification of small molecules that support human leukemia stem cell activity ex vivo. Nature Methods, 11(4):436-42.

Pearce, D. J., Taussig, D., Simpson, C., et al. (2005). Characterization of cells with a high aldehyde dehydrogenase activity from cord blood and acute myeloid leukemia samples. Stem Cells, 23(6), 752-760.

Peng, K. W., Ahmann, G. J., Pham, L., et al. (2001). Systemic therapy of myeloma xenografts by an attenuated measles virus. Blood, 98(7), 2002-2007.

Peng, Q., Cai, H., Sun, X., et al. (2013). Alocasia cucullata exhibits strong antitumor effect in vivo by activating antitumor immunity. PloS One, 8(9), e75328.

Porter, D. L., Levine, B. L., Kalos, M., et al. (2011). Chimeric antigen receptor-modified T cells in chronic lymphoid leukemia. New England Journal of Medicine, 365(8), 725-733.

Powell, J. D., Pollizzi, K. N., Heikamp, E. B., et al. (2012). Regulation of immune responses by mTOR. Annual Review of Immunology, 30, 39-68.

Rabinovich, G. A., Gabrilovich, D., \& Sotomayor, E. M. (2007). Immunosuppressive strategies that are mediated by tumor cells. Annual Review of Immunology, 25, 267-296.

Radoja, S., \& Frey, A. B. (2000). Cancer-induced defective cytotoxic T lymphocyte effector function: another mechanism how antigenic tumors escape immune-mediated killing. Molecular Medicine, 6(6), 465.

Rais, Y., Zviran, A., Geula, S., et al. (2013). Deterministic direct reprogramming of somatic cells to pluripotency. Nature, 502(7469), 65-70.

Raval, R. R., Sharabi, A. B., Walker, A. J., et al. (2014). Tumor immunology and cancer immunotherapy: summary of the 2013 SITC primer. Journal for ImmunoTherapy of Cancer, 2(1), 1-11.

Reiman, J. M., Knutson, K. L., \& Radisky, D. C. (2010). Immune promotion of epithelialmesenchymal transition and generation of breast cancer stem cells. Cancer Research, 70(8), 3005-3008. 
Reiners, K. S., Kessler, J., Sauer, M., et al. (2013). Rescue of impaired NK cell activity in hodgkin lymphoma with bispecific antibodies in vitro and in patients. Molecular Therapy, 21(4), 895-903.

Romero, A. I., Thorén, F. B., Aurelius, J., et al. (2009). Post-consolidation immunotherapy with histamine dihydrochloride and interleukin-2 in AML. Scandinavian Journal of Immunology, 70(3), 194-205.

Rosenkilde, M. M., Gerlach, L. O., Jakobsen, J. S., et al. (2004). Molecular mechanism of AMD3100 antagonism in the CXCR4 receptor Transfer of binding site to the CXCR3 receptor. Journal of Biological Chemistry, 279(4), 3033-3041.

Russell, S. J., Federspiel, M. J., Peng, K. W., et al. (2014). Remission of Disseminated Cancer After Systemic Oncolytic Virotherapy. Mayo Clinic Proceedings, 89(7), 926-933.

Sahlgren, C., Gustafsson, M. V., Jin, S., et al. (2008). Notch signaling mediates hypoxiainduced tumor cell migration and invasion. Proceedings of the National Academy of Sciences, 105(17), 6392-6397.

Sarry, J. E., Murphy, K., Perry, R., et al. (2011). Human acute myelogenous leukemia stem cells are rare and heterogeneous when assayed in NOD/SCID/IL2RYc-deficient mice. The Journal of Clinical Investigation, 121(1), 384.

Satoh, A., Bryant, S. V., \& Gardiner, D. M. (2008). Regulation of dermal fibroblast dedifferentiation and redifferentiation during wound healing and limb regeneration in the Axolotl. Development, Growth \& Differentiation, 50(9), 743-754.

Saxena, M., Stephens, M. A., Pathak, H., et al. (2011). Transcription factors that mediate epithelial-mesenchymal transition lead to multidrug resistance by upregulating $A B C$ transporters. Cell Death \& Disease, 2(7), e179.

Schmitz, F., Heit, A., Dreher, S., et al. (2008). Mammalian target of rapamycin (mTOR) orchestrates the defense program of innate immune cells. European Journal of Immunology, 38(11), 2981-2992.

Scotet, E., Martinez, L. O., Grant, E., et al. (2005). Tumor recognition following Vү9Vס2 $T$ cell receptor interactions with a surface F1-ATPase-related structure and apolipoprotein Al. Immunity, 22(1), 71-80.

Shang, Y., Li, Z., Li, H., et al. (2013). TIM-3 expression in human osteosarcoma: Correlation with the expression of epithelial-mesenchymal transition-specific biomarkers. Oncology Letters, 6(2), 490-494. 
Silzle, T., Randolph, G. J., Kreutz, M., \& Kunz-Schughart, L. A. (2004). The fibroblast: sentinel cell and local immune modulator in tumor tissue. International Journal of Cancer, 108(2), 173-180.

Smith, X., Schneider, H., Kohler, K., et al. (2013). The chemokine CXCL12 generates costimulatory signals in T cells to enhance phosphorylation and clustering of the adaptor protein SLP-76. Science Signaling, 6(286), ra65.

Smits, E. L., Anguille, S., Cools, N., et al. (2009). Dendritic cell-based cancer gene therapy. Human Gene Therapy, 20(10), 1106-1118.

Sousa, S., Afonso, N., Bensimon-Brito, A., et al. (2011). Differentiated skeletal cells contribute to blastema formation during zebrafish fin regeneration. Development, 138(18), 3897-3905.

Stewart, S., \& Stankunas, K. (2012). Limited dedifferentiation provides replacement tissue during zebrafish fin regeneration. Developmental Biology, 365(2), 339-349.

Stringaris, K., Sekine, T., Khoder, A., et al. (2014). Leukemia-induced phenotypic and functional defects in natural killer cells predict failure to achieve remission in acute myeloid leukemia. Haematologica, 99(5), 836-847.

Sun, L., Yao, Y., Liu, B., et al. (2012). MiR-200b and miR-15b regulate chemotherapyinduced epithelial-mesenchymal transition in human tongue cancer cells by targeting BMI1. Oncogene, 31(4), 432-445.

Sutherland, H. J., Lansdorp, P. M., Henkelman, D. H., et al. (1990). Functional characterization of individual human hematopoietic stem cells cultured at limiting dilution on supportive marrow stromal layers. Proceedings of the National Academy of Sciences, 87(9), 3584-3588.

Suzuki, N., Yamazaki, S., Yamaguchi, T., et al. (2013). Generation of engraftable hematopoietic stem cells from induced pluripotent stem cells by way of teratoma formation. Molecular Therapy. 21(7), 1424-1431.

Takahashi, K., \& Yamanaka, S. (2006). Induction of pluripotent stem cells from mouse embryonic and adult fibroblast cultures by defined factors. Cell, 126(4), 663-676.

Takahashi, K., Tanabe, K., Ohnuki, M., et al. (2007). Induction of pluripotent stem cells from adult human fibroblasts by defined factors. Cell, 131(5), 861-872.

Torossian, F., Anginot, A., Chabanon, A., et al. (2014). CXCR7 participates in CXCL12induced CD34+ cell cycling through $\beta$-arrestin-dependent Akt activation. Blood, 123(2), 191-202. 
Tran, E., Turcotte, S., Gros, A., et al. (2014). Cancer immunotherapy based on mutationspecific CD4+ T Cells in a patient with epithelial cancer. Science, 344(6184), 641-645.

Tsubooka, N., Ichisaka, T., Okita, K., et al. (2009). Roles of Sall4 in the generation of pluripotent stem cells from blastocysts and fibroblasts. Genes to Cells, 14(6), 683-694.

Tweedell, K. S. (2010). The urodele limb regeneration blastema: the cell potential. The Scientific World Journal, 10, 954-971.

Vali, B., Jones, R. B., Sakhdari, A., et al. (2010). HCV-specific T cells in HCV/HIV coinfection show elevated frequencies of dual Tim-3/PD-1 expression that correlate with liver disease progression. European Journal of Immunology, 40(9), 2493-2505.

van den Boorn, J. G., \& Hartmann, G. (2013). Turning Tumors into Vaccines: Co-opting the Innate Immune System. Immunity, 39(1), 27-37.

Vanneman, M., \& Dranoff, G. (2012). Combining immunotherapy and targeted therapies in cancer treatment. Nature Reviews Cancer, 12(4), 237-251.

Vizcardo, R., Masuda, K., Yamada, D., et al. (2013). Regeneration of Human Tumor Antigen-Specific T Cells from iPSCs Derived from Mature CD8+ T Cells. Cell Stem Cell, 12(1), 31-36.

Wen-Li, Z., Jian, W., Yan-Fang, T., et al. (2012). Inhibition of the ecto-beta subunit of F1F0-ATPase inhibits proliferation and induces apoptosis in acute myeloid leukemia cell lines. Journal of Experimental \& Clinical Cancer Research, 31(1), 92.

Wiener, Z., Kohalmi, B., Pocza, P., et al. (2007). TIM-3 is expressed in melanoma cells and is upregulated in TGF- $\beta$ stimulated mast cells. Journal of Investigative Dermatology, 127(4), 906-914.

Wiseman, D. H., Greystoke, B. F., \& Somervaille, T. C. P. (2013). The variety of leukemic stem cells in myeloid malignancy. Oncogene, 33(24), 3091-3098.

Wu, F. H., Yuan, Y., Li, D., et al. (2010). Endothelial cell-expressed Tim-3 facilitates metastasis of melanoma cells by activating the NF-kB pathway. Oncology Reports, 24(3), 693-699.

Yang, Z. Z., Grote, D. M., Ziesmer, S. C., et al. (2012). IL-12 upregulates TIM-3 expression and induces $T$ cell exhaustion in patients with follicular B cell non-Hodgkin lymphoma. The Journal of Clinical Investigation, 122(4), 1271-1282.

Yoshida, Y., Takahashi, K., Okita, K., et al. (2009). Hypoxia enhances the generation of induced pluripotent stem cells. Cell Stem Cell, 5(3), 237-241. 
Zhao, C., Yasumura, D., Li, X., et al. (2011). mTOR-mediated dedifferentiation of the retinal pigment epithelium initiates photoreceptor degeneration in mice. The Journal of Clinical Investigation, 121(1), 369-383.

Zhong, R. K., Loken, M., Lane, T. A., et al. (2006). CTLA-4 blockade by a human MAb enhances the capacity of AML-derived $D C$ to induce T-cell responses against AML cells in an autologous culture system. Cytotherapy, 8(1), 3-12.

Zhou, Q., Munger, M. E., Veenstra, R. G., et al. (2011). Coexpression of Tim-3 and PD-1 identifies a CD8+ T-cell exhaustion phenotype in mice with disseminated acute myelogenous leukemia. Blood, 117(17), 4501-4510.

Zhuang, X., Zhang, X., Xia, X., et al. (2012). Ectopic expression of TIM-3 in lung cancers a potential independent prognostic factor for patients with NSCLC. American Journal of Clinical Pathology, 137(6), 978-985.

Zitvogel, L., Apetoh, L., Ghiringhelli, F., et al. (2008). Immunological aspects of cancer chemotherapy. Nature Reviews Immunology, 8(1), 59-73.

Zitvogel, L., Tesniere, A., \& Kroemer, G. (2006). Cancer despite immunosurveillance: immunoselection and immunosubversion. Nature Reviews Immunology, 6(10), 715-727. 


\title{
CURRICULUM VITAE
}

\author{
Audrey Nadine Jajosky \\ 1340 Winona Avenue \\ Morgantown, WV 26505 \\ AJajosky@gmail.com (508) 579-0794
}

\section{EDUCATION}

West Virginia University (WVU), Morgantown, WV

Ph.D. in Cancer Cell Biology, 2014

MD (pending)

Worcester Polytechnic Institute (WPI), Worcester, MA

B.S. in Biology and Biotechnology with High Distinction, 2007

Concentration in Molecular Biology and Genetics

Minor in Biochemistry

\section{RESEARCH EXPERIENCE}

\section{Doctoral Dissertation:}

"An Overiew of Acute Myeloid Leukemia and Immunology: (i) Concepts and Therapeutic Strategies and (ii) RepSox as a Candidate Cell-Engineering Tool"

Advisor: Laura F. Gibson, Ph.D., WVU

\section{Undergraduate Projects:}

- Sufficiency: "Humor as Medicine" Advisor: Daniel G. Gibson, Ph.D., WPI

- Interactive Qualifying Project: "GE-NBC TV Humor-in-Healthcare Initiative" Advisor: Lorraine D. Higgins, Ph.D., WPI

- Major Qualifying Project: "Minimal Requirements for Assembly of a Stable Inner Membrane Platform for Vibrio cholerae Pilus Biogenesis"

Advisors: Ronald Taylor, Ph.D., Dartmouth Medical School and Jill Rulfs, Ph.D., WPI 


\section{Summer Experiences:}

Carnegie Mellon University Scholar, Pittsburgh, PA 2002

Volunteer Breast Cancer Research Assistant in WPI Biology Department 2003

Duke University Biomedical Research Institute, Durham, NC 2004

Medical Enrichment Program at Medical College of Georgia, Augusta, GA 2005

Microbiology/Genetics Research, Dartmouth Medical School, Hanover, NH 2006

MD/PhD rotation in cancer lab of Steven Frisch, Ph.D. Morgantown, WV 2007

MD/PhD rotation in cardiology lab of Mitchell Finkel, MD, Morgantown, WV 2008

\section{WORK}

Resident Advisor for students living on WPI campus 2004 -2007

Peer Writing Tutor

$2004-2007$

EMSEP Math \& Science Tutor

$2005-2006$

\section{LEADERSHIP}

Panelist for NIH Fellowship workshop for WVU graduate students 2011

Host of workshop on how to write an NIH Research Proposal 2010

Treasurer of Phi Sigma (National Biology Honor Society) 2005 - 2007

Member of EMSEP Advisory Board $2004-2007$

\section{PROFESSIONAL ASSOCIATIONS}

American Physician Scientist Association, WVU Chapter 2008 - present

The American Association for Cancer Research $\quad 2010-2011$

$\begin{array}{ll}\text { The Leadership Alliance } & 2006-2007\end{array}$

Women's Industry Network 2005 - 2007

\section{FELLOWSHIPS \& AWARDS}

Ruth L. Kirschstein Pre-Doctoral National Research Service Award 2011 - 2014 National Cancer Institute, National Institute of Health

$1^{\text {st }}$-place Poster at Van Liere \& WVU Health Sciences Center Research Day 2014

National Cancer Institute Supplemental Funding Award 2010 - 2011

Top-4 Finalist at WV state-wide STaR Science Symposium 2010

"High Distinction" Graduate of WPI 2007

WPI "Medical Professions Scholar" $\quad 2003-2007$

WPI "Excellence in Math, Science, and Engineering" Scholar 2003 - 2007

WPI Student "Woman-of-the-Year" (Ellen Knott Award) 2004 


\section{PRESENTATIONS \& POSTERS}

"Blood cell-spheres: can they help us develop better leukemia therapies?" Presentation, $1^{\text {st }}$ annual Osborn Hematological Malignancy Retreat, Morgantown, WV, August 2010.

Jajosky, A. N., Craig, M. D., Martin, K. H., Zhang, L., and Gibson, L. F. Blood cell-spheres: growth, description, and therapeutic potential. Poster. National MRS-AACR Joint Conference on Metastasis and the Tumor Microenvironment, Philadelphia, PA, September 2010.

Jajosky, A. N., Craig, M. D., Martin, K. H., Zhang, L., and Gibson, L. F. Growing normal and leukemia blood cell-spheres: the quest for stem cells and effective leukemia treatments. Poster, Science, Technology, and Research (STaR) Symposium, Huntington, WV, September 2010.

Jajosky, A. N., Craig, M. D., Martin, K. H., Zhang, L., and Gibson, L. F. Growing normal and leukemia blood cell-spheres: the quest for stem cells and effective leukemia treatments. Poster, $14^{\text {th }}$ Annual Meeting of the Translational Research Cancer Centers Consortium, Seven Springs, PA, February 2011.

Jajosky, A. N., Craig, M. D., Martin, K. H., Zhang, L., and Gibson, L. F. Growing normal and leukemia blood cell-spheres: the quest for stem cells and effective leukemia treatments. Poster, E.J. Van Liere Memorial Convocation and Health Sciences Research Day, Morgantown, WV, March 2011.

"Can we de-differentiate leukemia cells into the stem/progenitor cells we want to target therapeutically?" Presentation, $2^{\text {nd }}$ annual Osborn Hematological Malignancy Retreat, Morgantown, WV, August 2011.

Jajosky, A. N. and Gibson, L. F. The Tgf- $\beta$ inhibitor "RepSox" slows the decay of leukemic progenitors in vitro. Presentation and poster, $15^{\text {th }}$ Annual Meeting of the Translational Research Cancer Centers Consortium, Seven Springs, PA, February 2012.

Jajosky, A. N. and Gibson, L. F. A way to isolate leukemic progenitors? Poster, E.J. Van Liere Memorial Convocation and Health Sciences Research Day, Morgantown, WV, March 2012.

"Evaluating in vitro environments that may facilitate the engineering of leukemic stem cell-targeted immunotherapies." Presentation, $3^{\text {rd }}$ annual Osborn Hematological Malignancy Retreat, Morgantown, WV, August 2012.

Jajosky, A. N., Coad, J. E., Vos, J. A., Martin, K. H., Senft, J. R., Wenger, S. L., and Gibson, L. F. Potential utility of RepSox, a cellular reprogramming tool, for engineering "leukemic stem cell"targeted immunotherapies in vitro. Poster. E.J. Van Liere Memorial Convocation and Health Sciences Research Day, Morgantown, WV, February 2014. 


\section{PUBLICATIONS}

Jajosky, A. N., Coad, J. E., Vos, J. A., Martin, K. H., Senft, J. R., Wenger, S. L., \& Gibson, L. F. (2014). RepSox slows decay of CD34 ${ }^{+}$acute myeloid leukemia cells and decreases $\mathrm{T}$ cell immunoglobulin mucin-3 expression. Stem Cells Translational Medicine, 3(7), 836-848.

Lewis, J. W., Talkington, W. J., Walker, N. A., Spirou, G. A., Jajosky, A., Frum, C., and Brefczynski-Lewis, J. A. (2009). Human cortical organization for processing vocalizations indicates representation of harmonic structure as a signal attribute. The Journal of Neuroscience, 29(7), 2283-2296. 